ESAIM: PROCEEDINGS, December 2011, Vol. 34, p. 1-96

E. Cancès, V. Louvet and M. Massot, Editors

\title{
ADAPTIVE MULTIRESOLUTION METHODS
}

\author{
Margarete O. Domingues ${ }^{1}$, Sônia M. Gomes ${ }^{2}$, Olivier Roussel ${ }^{3}$ and \\ KAI SCHNEIDER ${ }^{4}$
}

\begin{abstract}
These lecture notes present adaptive multiresolution schemes for evolutionary PDEs in Cartesian geometries. The discretization schemes are based either on finite volume or finite difference schemes. The concept of multiresolution analyses, including Harten's approach for point and cell averages, is described in some detail. Then the sparse point representation method is discussed. Different strategies for adaptive time-stepping, like local scale dependent time stepping and time step control, are presented. Numerous numerical examples in one, two and three space dimensions validate the adaptive schemes and illustrate the accuracy and the gain in computational efficiency in terms of CPU time and memory requirements. Another aspect, modeling of turbulent flows using multiresolution decompositions, the so-called Coherent Vortex Simulation approach is also described and examples are given for computations of three-dimensional weakly compressible mixing layers. Most of the material concerning applications to PDEs is assembled and adapted from previous publications [27,31,32,34,67,69].
\end{abstract}

\section{INTRODUCTION}

Adaptive numerical techniques are very attractive tools to solve systems of nonlinear partial differential equations (PDEs) without wasting computational resources while preserving the accuracy of the solution. PDEs naturally arise from mathematical modeling of chemical-physical problems encountered in many applications, like in meteorology or chemical industry. In turbulent reactive or non-reactive flows, for instance, the solutions of these PDEs usually exhibit several magnitudes of active spatial and temporal scales. Typically these scales are not uniformly distributed in the space-time domain, thus efficient numerical discretizations could take advantage of this property. Space-time adaptivity allows thus to reduce the computational complexity with respect to uniform discretizations, while controlling the accuracy of the adaptive discretization.

Different approaches have been investigated to define adaptive space discretizations, some emerge from ad hoc criteria, others call for more sophisticated a posteriori error estimators using control strategies by solving computational expensive adjoint problems [5,76]. Adaptive mesh refinement (AMR) methods introduced by Berger and Oliger [8] are now widely used for many applications using structured or unstructured grids, see

\footnotetext{
1 Laboratório Associado de Computação e Matemática Aplicada (LAC), Coordenadoria dos Laboratórios Associados (CTE), Instituto Nacional de Pesquisas Espaciais (INPE), Av. dos Astronautas 1758, 12227-010 São José dos Campos, São Paulo, Brazil (e-mail: margarete@lac.inpe.br)

2 Instituto de Matemática, Estatística e Computação Científica (IMECC), Universidade Estadual de Campinas (Unicamp), R. Sérgio Buarque de Holanda 651, 13083-970 Campinas, São Paulo, Brazil (e-mail: soniag@ime.unicamp.br)

3 Centre de Mathématiques et Leurs Applications (CMLA), Ecole Normale Supérieure de Cachan, 61 avenue du President Wilson, 94235 Cachan cedex, France (e-mail: o_roussel@yahoo.fr)

4 Laboratoire de Mécanique, Modelisation et Procédés Propres (M2P2), CNRS, and Centre de Mathétiques e d'Informatique (CMI), Université de Provence, 39 rue F. Joliot-Curie, 13451 Marseille Cedex 13, France e-mail: kschneid@cmi.univ-mrs.fr)
}

(c) EDP Sciences, SMAI 2011 
e.g. $[6,7]$. However, the data compression rate is high where the solution is almost constant, but remains low where the solution is smooth.

Multiresolution based schemes (MR) have been first developed by Harten [46,47] for conservation laws. Now they are known to yield an appropriate framework to construct adaptive schemes for hyperbolic conservation laws. Harten's approach has then been extended and further developed in different directions by Cohen et al. [17], Kaibara and Gomes [51], Chiavassa and Donat [13], Müller [58], Roussel et al. [66,69]. The main idea of the MR method is to use a multiresolution data representation. The decay of the MR coefficients yields information on local regularity of the solution. Therewith the truncation error can be estimated and coarser grids can be used in regions where this error is small and the solution is smooth. An adaptive grid can be introduced by suitable thresholding of the multiresolution representation where only significant coefficients are retained. Hence a given discretization on a uniform mesh can be accelerated considerably as the number of costly flux evaluations is significantly reduced, without loosing the accuracy of the discretization. The memory requirements could also be reduced, for example using a dynamic tree data structure or hash tables. An overview of the different MR methods can be found, e.g. in the books of Cohen [14] and Müller [58].

A main drawback of most of these space-adaptive methods, which mostly employ explicit or semi-explicit time discretizations, is that the finest spatial grid size imposes a small time step in order to fulfill the stability criterion of the time scheme. Hence, for extensive grid refinement with a huge number of refinement levels, a very small size of the time step is implied. To overcome this difficulty different strategies have been pursued to introduce adaptive time stepping for space adaptive discretizations of PDEs. Osher and Sanders introduced local time stepping for one dimensional scalar conservation laws where the space discretization is non-uniform but fixed [61]. Extensions of this approach have been presented in Dawson and Kirby [22] for second order Runge-Kutta schemes using a predictor-corrector type local time stepping, which has been further improved by Tang and Warnecke [78]. Space-time mesh refinement for the one-dimensional wave equation based on the conservation of a discrete energy is proposed by Collino et al. [19,20]. In [37] a local time stepping algorithm for discontinuous Galerkin methods is presented by Dumbser et al. Each element uses its optimal time step given by the local stability condition without requiring synchronization between the elements. In [41] Ferm and Löstedt consider adaptive space-time strategies for hyperbolic problems in one space dimension in the context of AMR methods. The grid adaptation is done by blocks and are based on the control of local discretization errors in space, which are estimated by comparing the space discretization on two different grids. The adaptation in time chooses the time step according to the step size of each grid block combined with a time step control strategy.

In the context of adaptive multiresolution and wavelet methods, Bacry et al. [4] first introduced a scaledependent time step. They applied this method to linear parabolic equations and to the Burgers equation. A stability analysis of this scheme has been conducted for the heat equation in [12]. It is shown that the adaptive time stepping strategy does not affect the stability of the scheme.

More recently, Müller and Stiriba [59] presented a fully adaptive multiresolution finite volume scheme with a locally varying time stepping. For time discretization one stage methods, either explicit or implicit Euler schemes are used. A linear combination, leading to a Crank-Nicholson scheme, yields second order accuracy. Applications for one dimensional conservation laws are discussed to illustrate the efficiency and accuracy of the scheme. A pure space-time Galerkin approach for viscous Burgers equation where the time axis is treated like a space direction has been introduced by Alam et al. [2]. Results for one space dimension look promising, however the extension of this method to higher dimensions seems questionable as it could be expensive in memory storage.

The aim of the lecture notes is to present in a concise way adaptive multiresolution methods for evolutionary PDEs. Most of the presented material is adapted from and based on journal publications [27,31,32,34,67,69]. In addition to space adaptivity we show that local scale-dependent time stepping can yield additional speed-up. The idea is hereby to introduce at large scales larger time steps without violating the stability condition of the explicit time scheme, which results in less flux evaluations due to larger time steps.

In the following three kinds of adaptive strategies are considered to speed up adaptive multiresolution schemes in the finite volume context. 
a. Space adaptivity (MR): Finite volume (FV) discretizations on regular grids are applied on dynamically adapted grids. Space adaptivity is considered in the multiresolution (MR) context of wavelet analysis for cell averages. The main idea of such a MR scheme is to use the decay of the wavelet coefficients to obtain information on local regularity of the solution. Therewith, coarser grids can be used in regions where these coefficients are small and the solution is smooth, while fine grids are used where the coefficients are significant and the solution has strong variations. For an efficient MR representation, we adopt a data structure which is organized as a dynamic graded tree, as proposed in [69].

b. Time step control (CTS): The time integration is performed with variable time steps, where the time step size selection is based on estimated local truncation errors obtained by the computation of two solutions with embedded ODE solvers of different orders.

c. Local time stepping (LTS): The time evolution uses scale-dependent time steps. Instead of evolving the solution with a single time step $\Delta t$ on all grid cells, computational work is saved if the solution is integrated with different time steps, according to each cell scale: if $\Delta t$ is used for the cells in the finest level, then a double time step $2 \Delta t$ is used in the coarser level with double spacing. Required missing values in ghost cells are interpolated in intermediary time levels.

An analysis of the performance of the following adaptive multiresolution schemes is presented:

- MR: adaptive multiresolution scheme with the same constant time step at each every scale;

- MR/CTS: MR scheme with time step control, but the same time step at each each scale;

- MR/LTS: MR scheme with scale dependent local time stepping, but remaining constant in time;

- MR/CTS/LTS: MR scheme with time step control and local time stepping.

We also review the Sparse Point Representation method (SPR) which is concerned with adaptive finite difference schemes using interpolating multiresolution analysis [31,50,63]. Particularly, we describe a grid adaptation by blocks, as in the AMR method [8], but using wavelet coefficients as refinement indicators. This procedure combines ideas of both AMR and MR methods, and is convenient for parallelism purposes.

Application of the different adaptive schemes are given for various equations in one, two and three space dimensions. We demonstrate their accuracy and computational efficiency in terms of CPU time and memory requirements. Finally, we present a modeling part using the multiresolution representation to reduce the number of degrees of freedom for computing turbulent flows. The Coherent Vortex Simulation (CVS), originally introduced by Farge \& Schneider [38,39], is described here for weakly compressible flows. For an overview on multiresolution techniques for computational fluid dynamics we refer the reader to a recent review of Schneider \& Vasilyev [73].

The present lecture notes are organized as follows. In Section 2, the multiresolution representation framework is described, including Harten's approaches for point values and cell averages, and the lifting methodology. General adaptation concepts for evolutionary PDE using MR techniques are also summarized. Section 3 is dedicated to SPR method. Then, the coupling of finite volume schemes with adaptive multiresolution strategies is described in Section 4.1. Dynamical tree data structures are discussed, conservative flux evaluations are described and an error analysis is performed for linear equations, which yields an estimate for the threshold. Different time adaptive strategies are then exposed, and their coupling with the multiresolution scheme is detailed. In Section 5, various adaptive computations for different PDE are presented. We show results for convection-diffusion equations, for compressible Euler equations, for reaction-diffusion equations in one, two and three space dimensions, and also CVS computations of turbulent mixing layers. For numerous cases, the accuracy, CPU time and memory compression are discussed. Finally, conclusions are drawn and some perspectives for future investigations are discussed. 


\section{Multiresolution Representation}

In a multiscale framework, the information is organized at different scale levels $0 \leq j \leq J$. Typically, at a certain level $j$, the discrete data $\mathbf{f}^{j}=\left(f_{j, k}\right)$ is set by samples $f_{j, k}$ of a function $f$. In wavelet analysis, the details between two consecutive level $j$ and $j+1$ are kept in wavelet coefficients $\mathbf{d}_{j}=\left(d_{j, k}\right)$. The index $k$ is associated with a space location where the samples or the details are taken. For instance, as described in Section 2.1, the samples may obtained by point values or by cell averages in a hierarchy of grids, and the details $d_{j, k}$ are considered as errors of interpolation when the point values (or the cell averages) at the new points (or new cells) at level $j+1$ are predicted using the coarse information $\mathbf{f}_{j}$.

The main tools in a multiresolution context are appropriate one-to-one inter-level transformations

$$
\mathbf{f}_{j+1} \Leftrightarrow\left(\mathbf{f}_{j}, \mathbf{d}_{j}\right) .
$$

Using the information $\mathbf{f}_{j+1}$ at a finer level $j+1$, the output of the direct transformation contains the information $\mathbf{f}_{j}$ in the next coarser level, and the details $\mathbf{d}_{j}$. Conversely, given the coarser information $\mathbf{f}_{j}$ and the details $\mathbf{d}_{j}$, the finer information $\mathbf{f}_{j+1}$ is recovered by the inverse transformation. These transformations are required to satisfy some fundamental properties: localization, cancellation of polynomials and stability. Localization means that the computation of an entry in $\mathbf{f}_{j}$ or $\mathbf{d}_{j}$ requires the knowledge of a finite number of information from $\mathbf{f}_{j+1}$ which are localized in space close to the corresponding location. In order to have good data compression, the details $\mathbf{d}_{j}$ should vanish for polynomials up to a certain degree. This means that, in regions of smoothness, the wavelet coefficients are insignificant and can be neglected. Close to singularities, where the details are important, they need to be kept. Stability is a crucial property that guaranties that any perturbation introduced into the data at any scale level is not amplified without control under the interactive application of the multilevel transformations

$$
\mathbf{f}_{J} \Leftrightarrow \mathbf{f}_{J}^{M R}=\left(\mathbf{f}_{0}, \mathbf{d}_{0}, \ldots, \mathbf{d}_{J-1}\right)
$$

In wavelet analysis, the MR direct and inverse transforms are usually associated to multiresolution representations of functional spaces of the form

$$
V_{J}=V_{0}+\sum_{j=0}^{J-1} W_{j}
$$

where $V_{j}$ denotes the space spanned by scaling functions and $W_{j}$ the spaces spanned by wavelets. Thus functions $f \in V_{J}$ can be expanded either in scaling functions, which yields a single scale representation

$$
f(x)=\sum_{k} f_{J, k} \Phi_{J, k}(x),
$$

or in wavelets, which yields a multiscale representation

$$
f(x)=\sum_{k} f_{0, k} \Phi_{0, k}(x)+\sum_{j=0}^{J-1} \sum_{k} d_{j, k} \Psi_{j, k}(x) .
$$

The scaling and wavelet coefficients

$$
f_{j, k}=<f, \Phi_{j, k}^{*}>, \quad d_{j, k}=<f, \Psi_{j, k}^{*}>
$$

are defined respectively as inner products with dual scaling function $\Phi_{j, k}^{*}$ and dual wavelets $\Psi_{j, k}^{*}$.

In the classical approach, the content of $\mathbf{f}_{j}$ comes from local information at uniformly sampled sites of a Cartesian grid $X_{j}$, and the inter-level transformations are relied on convolutions with low and high pass filters. For a more detailed discussion on wavelets which yield a functional framework of MR analysis, we refer to the books of Mallat [57] and Cohen [14]. 
We consider here the construction of multiresolution analyses using two other different approaches: the formulation by A. Harten [1,47], and the lifting methodology by [77].

Harten's general framework for the construction of multiresolution representations of data is based on the concepts of discretization, restriction and prediction operators. This framework is described in Section 2.1, where discretization by point values and cell averages are considered for uniform grids on the interval and on Cartesian grids in higher dimensions. As an illustration in non-Cartesian setting, we use Harten's approach to describe some examples of multiresolution analyses for the triangular geometry.

In the lifting methodology the idea is to start from a simple framework, Haar-like wavelets for instance, and to use the lifting scheme (or dual lifting) [77] to obtain a new multiresolution analysis with better performance. For instance, with wavelets higher degree of polynomial cancellation or conservation of data average within the scale levels. The basic ideas of this methodology are described in Section 2.2.

For the application that we have in mind, a fundamental property is that the wavelet coefficients can be used as regularity indicators, which is a direct consequence of the properties of localization and polynomial cancellation: where the function is smooth, the wavelets coefficients are not significant, while in contrast their magnitudes are relevant close to singularities or strong gradients. In the contexts of MR for point values or cell averages, the principle is to represent functions only by the point values (or cells) corresponding to their significant wavelet coefficients. For functions having localized singularities, it is expected to find few grid points (or coarse cells) on smooth regions and refined grids close to the irregularities. These sparse representations can be used in the numerical solution of PDEs to make some numerical schemes more efficient. The basic adaptation concepts for evolutionary PDEs are described in Section 2.3. More details and examples are presented in the following sections.

\subsection{Harten's approach}

In $[1,47]$, a general framework for the construction of multiresolution representations of data is presented, which is based on the concepts of discretization, restriction and prediction operators.

To illustrate this methodology, we start by considering the discretizations by point values and cell averages for uniform grids in the unit interval $\bar{\Omega}=[0,1]$. This methodology can be used in more general contexts, for grids in higher dimensions and irregular geometry.

\subsubsection{Multiresolution analyses for point values on uniform dyadic grids}

Consider the hierarchy of uniform dyadic grids unit interval

$$
X_{j}=\left\{x_{j, k}=k 2^{-j}, k=0, \cdots, 2^{j}\right\}
$$

with spacing $2^{-j}$. As shown in Figure 1, this means that to go from $X_{j}$ to a more refined grid $X_{j+1}$ we add to $X_{j}$ the new midpoints $(2 k+1) 2^{-j-1}$ between the old points $k 2^{-j}$ and $(k+1) 2^{-j}$, halving the step size.

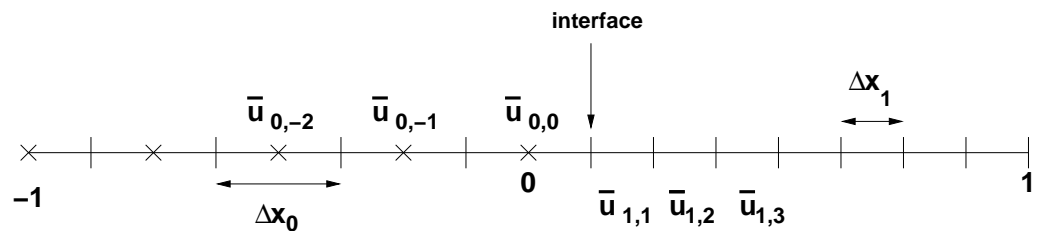

Figure 1. Dyadic grids on the interval.

In this geometry, at each scale level $j$, the discretization operator $\mathcal{D}_{j}: f \rightarrow \mathbf{f}_{j}$ associates to a continuous function $f(x)$ on the interval $[0,1]$ a vector $\mathbf{f}_{j}$ formed by its sample values $f_{j, k}=f\left(x_{j, k}\right)$ at the points in $X_{j}$.

For interchange of information between consecutive levels $j$ and $j+1$, restriction and prediction operators are required: 


$$
\begin{aligned}
& P_{j+1 \rightarrow j} \quad: \quad \mathbf{f}_{j+1} \rightarrow \mathbf{f}_{j} \\
& P_{j \rightarrow j+1} \quad: \quad \mathbf{f}_{j} \rightarrow \tilde{\mathbf{f}}_{j+1}
\end{aligned}
$$

Restriction (or projection) is the operation that goes from level $j+1$ to $j$ by just doing decimation, that is

$$
f_{j, k}=f_{j+1,2 k} .
$$

Prediction is a more subtle operation that, from the knowledge of its sample values at $X_{j}$, gives estimated values $\tilde{f}_{j+1, k} \approx f_{j+1, k}$ at the new odd locations in $X_{j+1}$.

\section{Interpolating predictions}

The main properties required for the interpolating prediction operator are:

- At the old points, the data are preserved: $\tilde{f}_{j+1,2 k}=f_{j+1,2 k}$.

- Localization: the computation of $\tilde{f}_{j+1,2 k+1}$ only requires the samples $f_{j, m}$ on a close neighbourhood of $x_{j+1,2 k+1}$.

- Reproduction of polynomials: the prediction is exact for polynomials of a prescribed degree.

A particular interest emerges regarding predictions by polynomial Lagrange interpolation. The most simple example is given by linear interpolation

$$
\tilde{f}_{j+1,2 k+1}=\frac{f_{j, k}+f_{j, k+1}}{2} .
$$

Generally, for schemes of order $M$, the idea is to define $\tilde{f}_{j+1,2 k+1}$ by the evaluation at $x_{j+1,2 k+1}$ of the polynomial $p(x)$ of degree $M-1$ that interpolates $f_{j, m}$ at the $M$ points $x_{j, m} \in X_{j}$ that are as close as possible to $x_{j+1,2 k+1}$. It is clear that such procedure will reproduce polynomials of degree $M-1$. The scheme is illustrated in Figure 2 for $M=2$ and 4 .

Far from the boundary of the interval, and for even $M=2 L$, we are in the classical iterative subdivision scheme by Deslauriers-Dubuc [28], where the interpolation stencils are centred around $x_{j+1,2 k+1}$, that is, $x_{j, m},-L \leq m \leq L$. As an example, we consider the case of cubic interpolation $(M=4)$. In the middle of the interval, the formula reads

$$
\tilde{f}_{j+1,2 k+1}=\frac{9}{16}\left(f_{j, k}+f_{j, k+1}\right)-\frac{1}{16}\left(f_{j, k-1}+f_{j, k+2}\right), \quad 0<k<2^{j} .
$$

Close to the left boundary, at $x_{j+1,1}$, we chose the interpolation stencil $x_{j, m}, m=0,1,2$ and 3 , resulting in

$$
\tilde{f}_{j+1,1}=\frac{5}{16} f_{j, 0}+\frac{15}{16} f_{j, 1}-\frac{5}{16} f_{j, 2}+\frac{1}{16} f_{j, 3},
$$

and on the right, at $x_{j+1,2^{j+1}-1}$, we chose the interpolation stencil $x_{j, 2^{j}-m}, m=0,1,2$ and 3 , and reverse the formula in Eq. 3 to get

$$
\tilde{f}_{j+1,2^{j+1}-1}=\frac{1}{16} f_{j, 2^{j}-3}-\frac{5}{16} f_{j, 2^{j}-2}+\frac{15}{16} f_{j, 2^{j}-1}+\frac{5}{16} f_{j, 2^{j}} .
$$




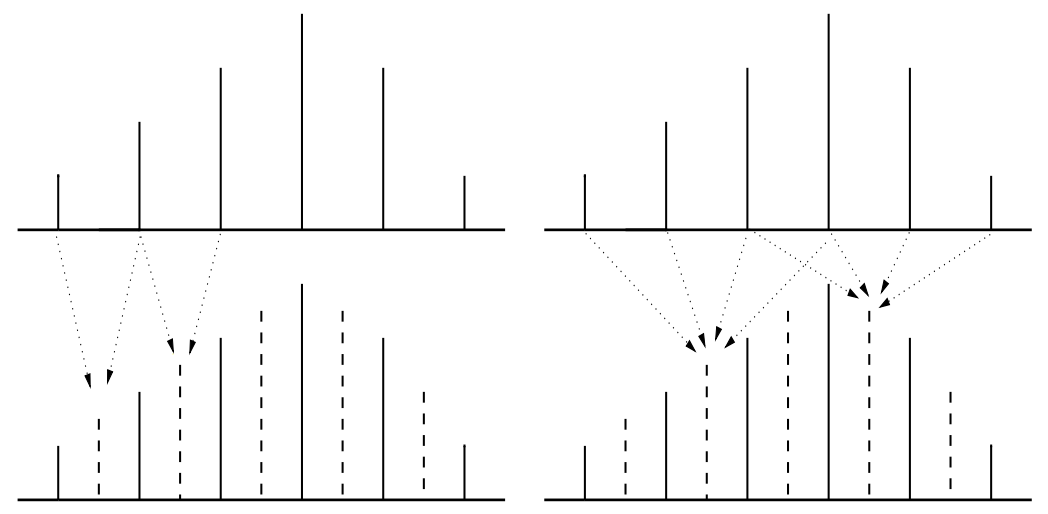

Figure 2. Prediction by linear (left) and cubic interpolation (right). The operation inserts approximated values in-between the old values.

\section{MR transformations}

Multiresolution analyses for point values are constructed using interpolating predictions. The difference of information between a scale level $j$ and the next upper level $j+1$ occurs at the new points $x_{j+1,2 k+1} \in X_{j+1} \backslash X_{j}$. Therefore, to measure these details, wavelet coefficients $d_{j, k}$ are defined as the prediction errors at such locations, as indicated in Figure 3.

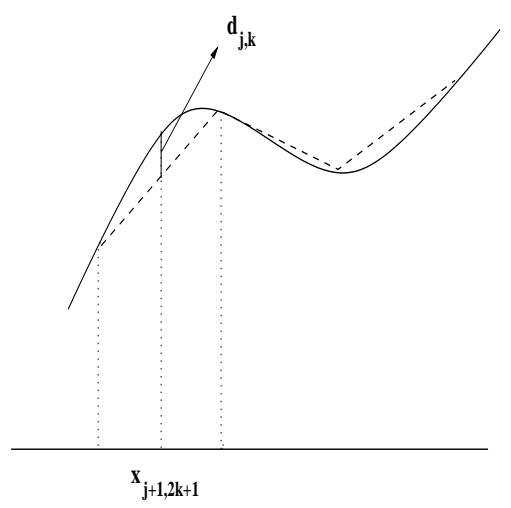

FigURE 3. Wavelet coefficient as the error in the linear prediction case.

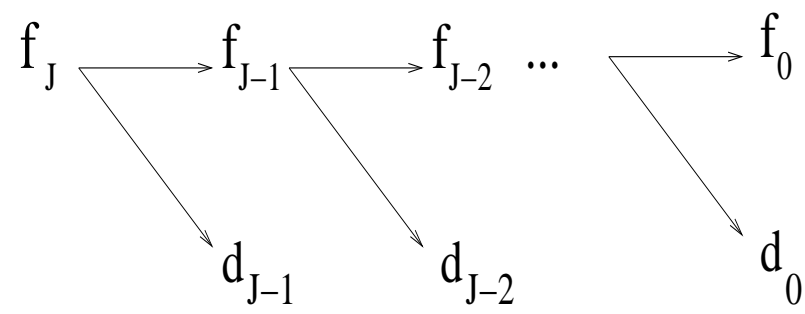

FIGURE 4. Structure of the analysis algorithm. 


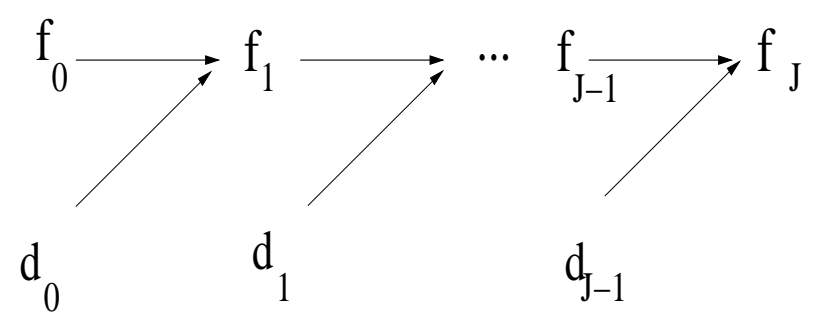

FiguRE 5. Structure of synthesis algorithm.

If we do this $J$ times, from level $J-1$ to 0 , we obtain the details $\mathbf{d}_{j}$, and the signal $\mathbf{f}_{0}$, at the coarsest level, as in Figure 4. In Figure 5, the structure of the reverse process is shown, which starts from $\mathbf{f}_{0}$ and $\mathbf{d}_{j}$, $j=0, \cdots J-1$, and recovers $\mathbf{f}_{J}$.

Analysis - $\mathbf{f}_{J} \rightarrow \mathbf{f}_{J}^{M R}=\left(\mathbf{f}_{0}, \mathbf{d}_{0}, \ldots, \mathbf{d}_{J-1}\right)$

For $j=J-1, \ldots, 0$, given $\mathbf{f}_{j+1}$ :

(1) Do restriction: $P_{j+1 \rightarrow j}: \mathbf{f}_{j+1} \rightarrow \mathbf{f}_{j}$

(2) Do prediction: $P_{j \rightarrow j+1}: \mathbf{f}_{j} \rightarrow \tilde{\mathbf{f}}_{j+1}$

(3) Compute prediction errors: $d_{j, k}=f_{j+1,2 k+1}-\tilde{f}_{\ell j+1,2 k+1}$

Synthesis $-\mathbf{f}_{J}^{M R}=\left(\mathbf{f}_{0}, \mathbf{d}_{0}, \ldots, \mathbf{d}_{J-1}\right) \rightarrow \mathbf{f}_{J}$

For $j=0, \ldots, J-1$, given $\mathbf{f}_{j}, \mathbf{d}_{j}$ :

(1) Do prediction: $P_{j \rightarrow j+1}: \mathbf{f}_{j} \rightarrow \tilde{\mathbf{f}}_{j+1}$

(2) Compute $\mathbf{f}_{j+1}$

$f_{j+1,2 k}=f_{j, k}$

$f_{j+1,2 k+1}=d_{j, k}+\tilde{f}_{j+1,2 k+1}$

Local regularity wavelet indicator

As interpolation errors, wavelet coefficients contain information about the local regularity of the analyzed function $f(x)$. For instance, denoting by $I_{j, k}$ the smallest interval containing the interpolation stencil used to compute $\tilde{f}_{j+1,2 k+1}$, the approximation theory by polynomial interpolation states that, for $1 \leq s \leq M-1$, there exists a constant $K=K(s, M)$ such that the estimation

$$
\left|d_{j, k}\right| \leq K 2^{-(s+1) j} \max _{\xi \in I_{j, k}}\left|f^{(s+1)}(\xi)\right|
$$

holds for functions $f \in C^{s}\left(I_{j, k}\right)$, having bounded derivative of order $s+1$.

This result can be used to estimate the degree of regularity of a function at a certain location by analyzing the decay rate of the magnitude of the wavelet coefficients associated to points in such zone. As an example, we consider the function

$$
f(x)= \begin{cases}8.1 e^{1 / 4} e^{-|x-1 / 2|}, & x \leq 1 / 4 \\ 9 e^{-|x-1 / 2|}, & 1 / 4 \leq x \leq 3 / 4 \\ e^{-|x-1 / 2|}\left(16 x^{2}-24 x+18\right), & x \geq 3 / 4\end{cases}
$$

which presents discontinuities at $\xi=1 / 4$, of $f^{\prime}$ at $\xi=1 / 2$, and of $f^{\prime \prime}$ at $\xi=3 / 4$. Figure 6 shows the plot of $f(x)$ on the left side. On the right side, the positions of the significant wavelet coefficients $\left|d_{j, k}\right| \leq 5 \times 10^{-4}$ are plotted in the position-scale plane, which indicates the location of the singularities. Now consider sufficiently localized 
$\delta$ neighborhoods of the singularities $\xi$, and define $A_{j}(\xi)=\max \left\{\left|d_{j, k}\right|:\left|x_{j+1,2 k-1}-\xi\right|<\delta\right\}$. Figure 7 shows the plots of $\log _{2} A_{j}(\xi)$, which have slopes approximately $\alpha=-0.0004,-1.0149$ and -1.9538 for $\xi=1 / 4,1 / 2$ and $3 / 4$, respectively, confirming the expected theoretical values $\alpha=0,-1$ and -2 .
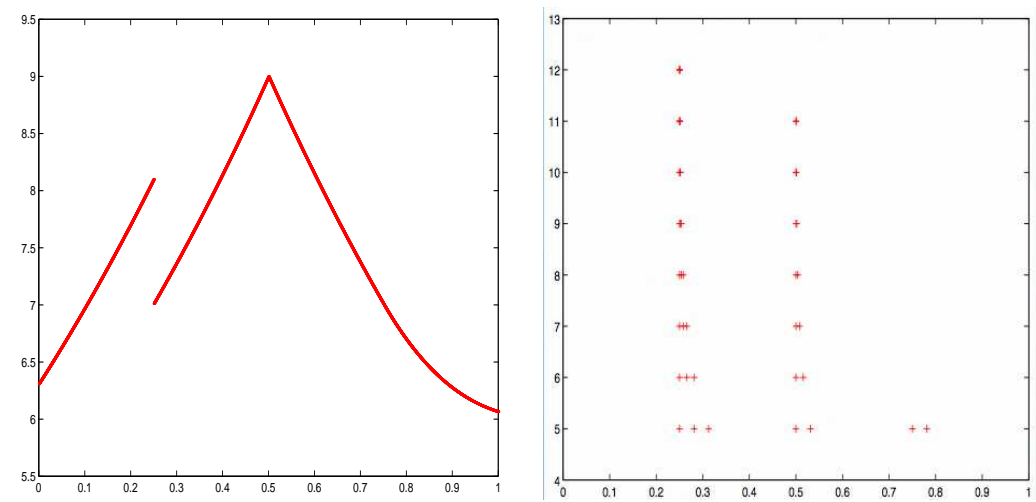

Figure 6 . The function $f(x)$ (left) and the position of the significant wavelet coefficients $\left|d_{j, k}\right| \leq 5 \times 10^{-4}$ (right).

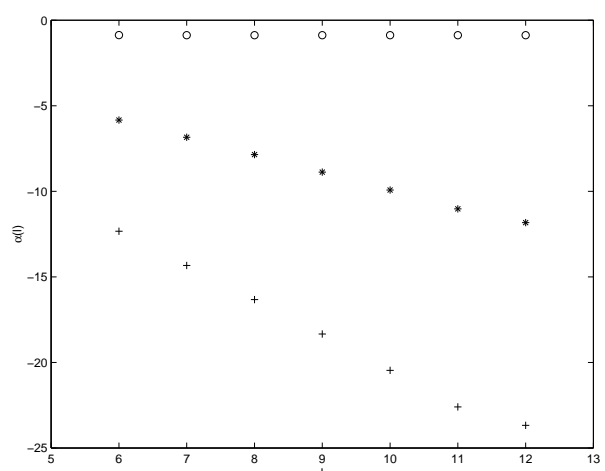

Figure 7. Local decay of the wavelet coefficients $\log _{2} A_{j}(\xi)$, for $\xi=1 / 4(\mathrm{o}), \xi=1 / 2(*)$, and $\xi=3 / 4(+)$.

\section{Functional context}

For discretization by point values, we can define multiresolution functional decompositions like described in (1). The grid point values may be interpreted as

$$
f_{j, k}=f\left(x_{j, k}\right)=<f, \Phi_{j, k}^{*}>,
$$

where $\Phi_{j, k}^{*}$ is the delta distribution at $x_{j, k}$. For the definition of the dual wavelets $\Psi_{j, k}^{*}$ such that

$$
d_{j, k}=f_{j+1,2 k+1}-\tilde{f}_{j+1,2 k+1}=<f, \Psi_{j, k}^{*}>
$$

it implies that

$$
\Psi_{j, k}^{*}=\Phi_{j, 2 k+1}^{*}-\sum_{m} h_{m}^{k} \Phi_{j, m}^{*},
$$


where $h_{m}^{k}$ are the interpolating prediction coefficients, such that

$$
\tilde{f}_{j+1,2 k+1}=\sum_{m} h_{m}^{k} f_{j, m}
$$

For instance, at locations in the center of the interval, we have $h_{m}^{k}=\{1 / 2,1 / 2\}$ for linear interpolation, and $h_{m}^{k}=\{-1 / 16,9 / 16,9 / 16,-1 / 16\}$ for cubic interpolation.

The definition of the primal basic functions $\Phi_{j, k}$ and $\Psi_{j, k}$ relies on the convergence of the prediction operator. An interpolating prediction is said to be convergent if for any starting sequence $s_{j}=\left(s_{j, k}\right)$, there exists a continuous function $s_{j}(x)$ such that $s_{j}\left(x_{\ell, k}\right)=s_{\ell, k}$, for $\ell \geq j$, where $s_{\ell}$ is defined by interactive subdivision $s_{\ell}=P_{\ell-1 \rightarrow \ell} s_{\ell-1}$. This property holds for predictions by polynomial Lagrange interpolation of degree $M-1$. Let the basic function $\Phi_{j, k}(x)$ be defined by the iterative subdivision scheme of the delta-sequence $s_{j, m}=\delta(k-m)$, which means that they satisfy the interpolating property

$$
\Phi_{j, k}\left(x_{j, m}\right)=\delta(k-m) .
$$

The scaling relation

$$
\Phi_{j, k}(x)=\sum_{m} c_{k}(m) \Phi_{j+1, m}(x),
$$

where $c_{k}(m)=\Phi_{j, k}\left(x_{j+1, m}\right)$, holds as a consequence of the fact that the functions in both sides coincide at $X_{j+1}$. Observe that the scaling coefficients are $c_{k}(2 m)=\delta(m-k)$, and $c_{k}(2 m+1)=h_{k}^{m}$. Furthermore, the following properties hold [35]:

- The regularity increases with interpolation degree $M-1$. For instance, $\Phi_{j, k}(x) \in C^{s}$, with $s>1.678$ and $s>2.272$ for $M=4,6$.

- Far from the boundaries, $\Phi_{j, k}(x)=\phi\left(2^{j} x-k\right)$, where $\phi=\varphi_{M}$ is the Dubuc-Deslauriers fundamental function

- $\Phi_{j, k}(x)$ has compact support, with support size $\left|\operatorname{supp}\left(\Phi_{j, k}\right)\right|=O\left(2^{-j}\right)$ increasing with $M$.

Figure 8 displays the scaling functions $\Phi_{j, k}(x)$ for linear and cubic interpolations, at level $j=5$, at the center of the interval. The interpolating scaling functions at level $j=5$, interacting with the left boundary, for cubic predictions, are shown in Figure 9. On the right side, they are obtained by reversing those of the left side.
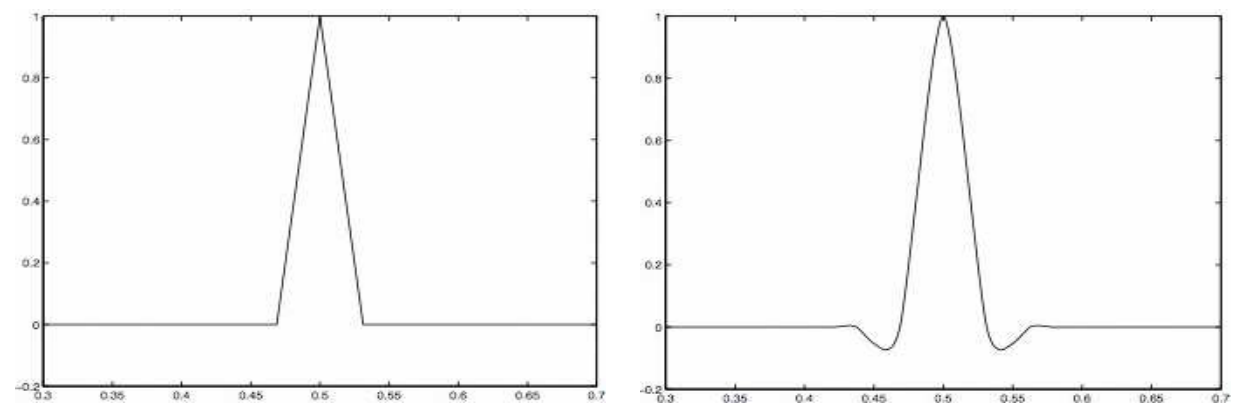

FIGURE 8. Interpolating scaling functions for linear (left side) and cubic predictions (right side), at level $j=5$.

By defining the functional subspaces

$$
V_{j}=\operatorname{span}\left\{\Phi_{j, k}(x), k=0, \cdots, 2^{j}\right\} \subset C[0,1],
$$



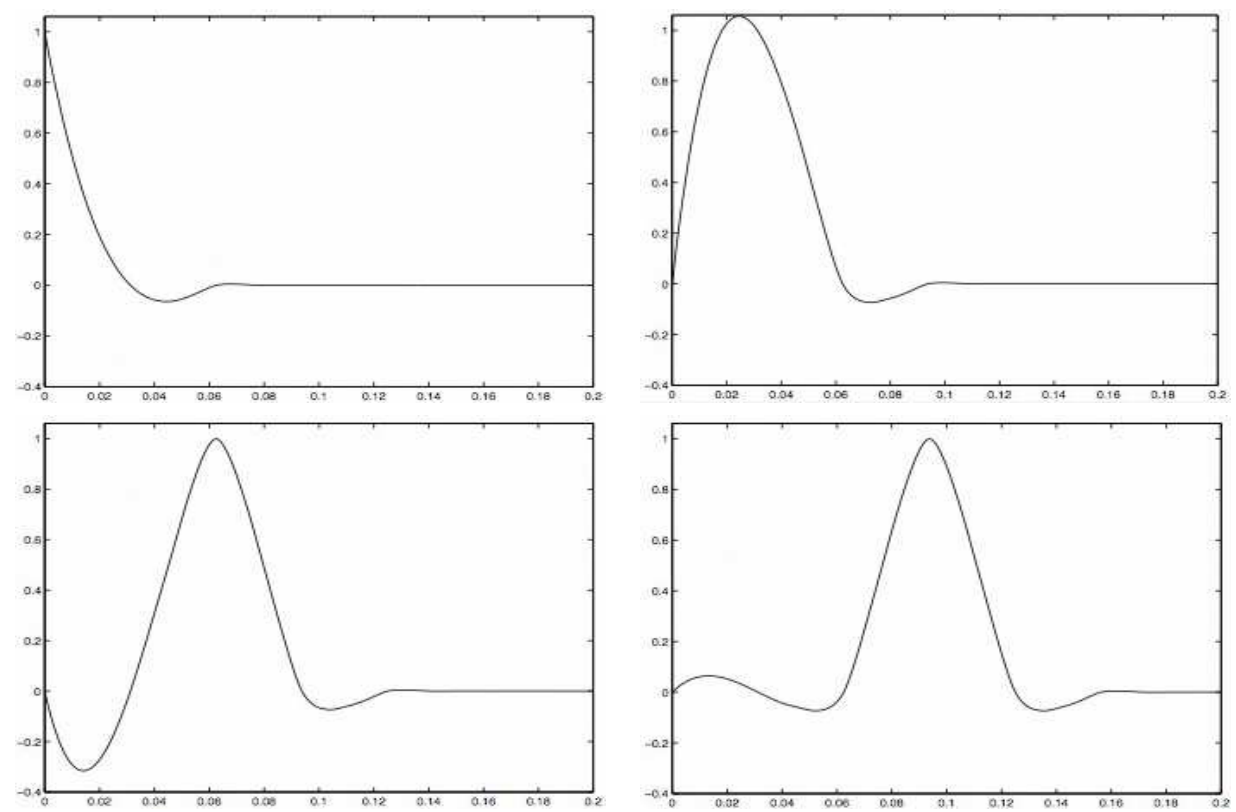

FIGURE 9. Interpolating scaling functions at level $j=5$, interacting with the left boundary, for cubic predictions.

by construction we have $V_{j} \subset V_{j+1}$. For functions $f \in C[0,1]$, we consider the sequence of approximations

$$
f_{j}(x)=\sum_{k} f_{j, k} \Phi_{j, k}(x)
$$

which correspond to interpolation operators of $f$ in $V_{j}$. It can be proved that

$$
\begin{aligned}
\left\|f_{J}\right\|_{\infty} & \leq C\|f\|_{\infty}, \\
\left\|f-f_{J}\right\|_{\infty} & \rightarrow 0, \text { as } J \rightarrow \infty .
\end{aligned}
$$

We consider the wavelets $\Psi_{j, k}(x)=\Phi_{j+1,2 k+1}(x)$ as the scaling functions associated with the points $x_{j+1,2 k+1} \in$ $X_{j+1} \backslash X_{j}$, and the space

$$
W_{j}=\operatorname{span}\left\{\Psi_{j, k}(x), k=0, \cdots, 2^{j}-1\right\},
$$

which is formed by those functions in $V_{j+1}$ that vanish on $X_{j}$. Therefore, it results that $V_{j+1}$ is the direct sum of the spaces $V_{j}$ and $W_{j}$. And since the difference between two consecutive reconstructions $f_{j+1}(x)-f_{j}(x)$ vanishes at $X_{j}$ and coincides with $d_{j, k}=f_{j+1,2 k+1}-\tilde{f}_{j+1,2 k+1}$ at $X_{j+1} \backslash X_{j}$. Then, the two-level direct-sum representation holds

$$
\begin{aligned}
V_{j+1} & =V_{j}+W_{j} \\
\sum_{k} f_{j+1, k} \Phi_{j+1, k} & =\sum_{k} f_{j, k} \Phi_{j, k}+\sum_{k} d_{j, k} \Psi_{j, k} .
\end{aligned}
$$

Figure 10 illustrates the two-level decomposition for interpolating multiresolution analysis for $j=4$, associated to linear prediction. 


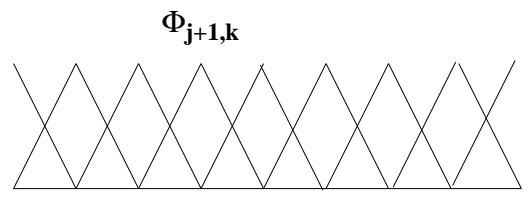

\section{WT}

IWT

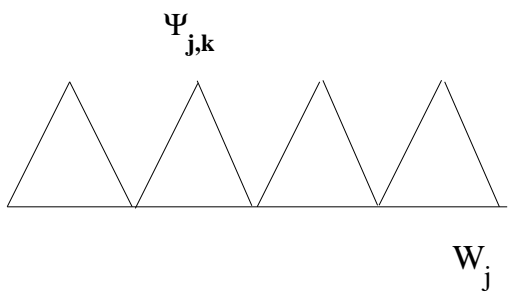

FiguRE 10. Two-level decomposition for interpolating multiresolution analysis for $j=4$, associated to linear prediction.

Interactively applying this decomposition, the MR direct (analysis) and inverse (synthesis) transforms are associated to the multiresolution representation

$$
\begin{aligned}
V_{J} & =V_{0}+\sum_{j=0}^{J-1} W_{j} \\
\sum_{k} f_{J, k} \Phi_{J, k}(x) & =\sum_{k} f_{0, k} \Phi_{0, k}(x)+\sum_{j=0}^{J-1} \sum_{k} d_{j, k} \Psi_{j, k}(x) .
\end{aligned}
$$

\section{Data compression}

Given the point values $\mathbf{f}_{J}$ on the finest level $\mathrm{J}$, after the analysis step of the MR transform, they can be represented as

$$
\mathbf{f}_{J}^{M R}=\left(\mathbf{f}_{0}, \mathbf{d}_{0}, \cdots, \mathbf{d}_{J-1}\right) .
$$

Given threshold parameters $\epsilon_{j}$, data compression is obtained by the thresholding operation

$$
d_{j, k}^{\epsilon}=\left\{\begin{array}{cc}
0 & \text { if }\left|d_{j, k}\right| \leq \epsilon_{j}, \\
d_{j, k} & \text { otherwise }
\end{array}\right.
$$

The two main properties of the prediction operator, namely, localization and polynomial reproduction, has an immediate consequence on the size of the wavelet coefficients in smooth regions. For instance, for functions as the one shown in Figure 6, it is expected that the compressed multiresolution representation

$$
\mathbf{f}_{J}^{M R, \epsilon}=\left(\mathbf{f}_{0}, \mathbf{d}_{0}^{\epsilon}, \ldots, \mathbf{d}_{J-1}^{\epsilon}\right)
$$

has a reduced number of non-vanishing terms.

For the applications described in Section 3, in the context of finite difference methods, the main idea is to use wavelet coefficients in a multiresolution representation of the point values of the solution to construct adaptive grids: use coarser cells in regions where the wavelet coefficients are small (and the solution is smooth) and refine where they are significant. This technique is known as SPR, and was introduced by M. Holmström [49]. 


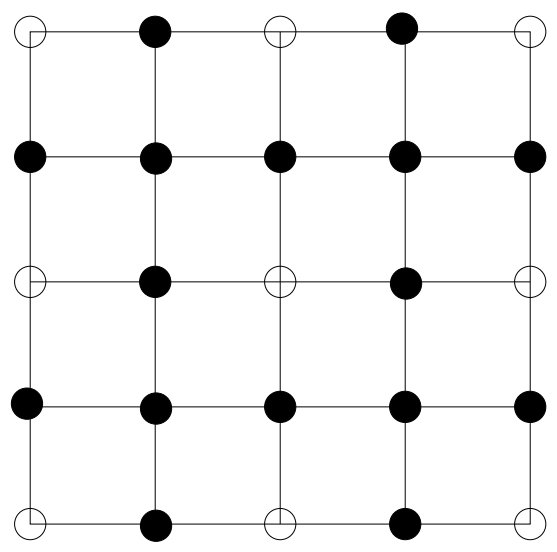

FigurE 11. Uniform dyadic grids: the symbol o represents points in $X_{j}$, while $\bullet$ represents points in $X_{j+1} \backslash X_{j}$.

\section{Stability}

There is another key aspect in wavelet compression: after the application of the inverse MR transform to the compressed data $\mathbf{f}_{J}^{M R, \epsilon}$, how do the reconstructed values $\mathbf{f}_{J}^{\epsilon}$ compare to the original ones? Note that a perturbation in the wavelet coefficients at level $j$ is transmitted to higher levels by successive predictions. Could such perturbations be amplified by this process without control?

In the context of convergent subdivision schemes, the stability result holds (see [47], Section 3.C)

$$
\left\|\mathbf{f}_{J}-\mathbf{f}_{J}^{\epsilon}\right\|_{\infty} \leq C \sum_{j=0}^{J-1} \epsilon_{j} .
$$

$M R$ analyses for point values in higher dimensions

In higher dimensions, multiresolution schemes for Cartesian grids can be obtained by tensor products of $1 \mathrm{D}$ schemes. For instance, on the square $[0,1] \times[0,1]$, starting from $X_{0}$, the grid at the coarsest level, the hierarchy of uniform grids $X_{j}, j \geq 1$ is

$$
X_{j}=\left\{x_{j,(k, m)}=\left(k 2^{-j}, m 2^{-j}\right), 0 \leq k \leq 2^{j}, 0 \leq m \leq 2^{j}\right\}
$$

As indicated in Figure 11, the dyadic refinement $X^{j+1}$, is obtained by the inclusion of new midpoints $\left(2 k \Delta_{x}^{\ell+1},(2 m+\right.$ $\left.1) \Delta_{y}^{\ell+1}\right),\left((2 k+1) \Delta_{x}^{j+1}, 2 m \Delta_{y}^{j+1}\right)$, and $\left((2 k+1) \Delta_{x}^{j+1},(2 m+1) \Delta_{y}^{j+1}\right)$. For the discretization

$$
f_{j,(k, m)}=f\left(x_{j,(k, m)}\right)
$$

we have the restriction operation

$$
f_{j,(k, m)}=f_{j+1,(2 k, 2 m)}
$$

The application of tensor product interpolating prediction corresponds to $1 \mathrm{D}$ interpolation in the $x$-direction to compute $f_{j+1,(2 k+1,2 m)}$, in the $y$-direction to compute $\tilde{f}_{j+1,(2 k, 2 m+1)}$. Finally, $\tilde{f}_{j+1,(2 k+1,2 m+1)}$ is obtained by the $1 \mathrm{D}$ interpolation in the $x$-direction of the already computed interpolated values $\tilde{f}_{j+1,(2 s, 2 m+1)}$. Then, wavelet coefficients are defined as prediction (interpolation) errors 


$$
\begin{aligned}
& d_{j,(k, m)}^{(1)}=f_{j+1,(2 k, 2 m+1)}-\tilde{f}_{j+1,(2 k, 2 m+1)} \\
& d_{j,(k, m)}^{(2)}=f_{j+1,(2 k+1,2 m)}-\tilde{f}_{j+1,(2 k+1,2 m)} \\
& d_{j,(k, m)}^{(3)}=f_{j+1,(2 k+1,2 m+1)}-\tilde{f}_{j+1,(2 k+1,2 m+1)}
\end{aligned}
$$

The 2D MR transformations inherit from the $1 \mathrm{D}$ setting the properties of cancellation of polynomials by the wavelet coefficients, localization and stability, and the wavelet coefficients can be used as local regularity indicators as well. This principle is used in the construction of 2D SPR of functions: only the point values corresponding to significant wavelet coefficients are kept in the adaptive grids. Figure 12 illustrates this technique.
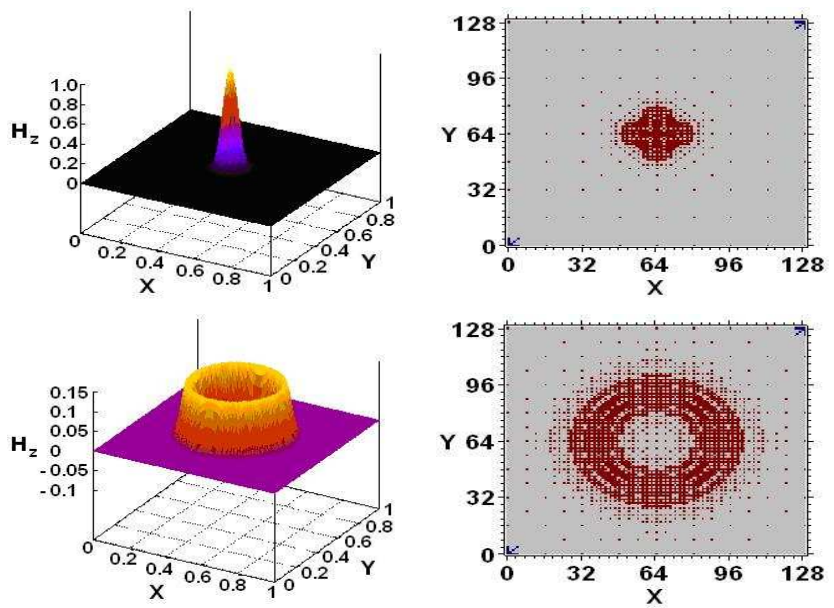

Figure 12. Example of SPR grids.

2.1.2. Multiresolution analyses for cell averages on uniform dyadic grids

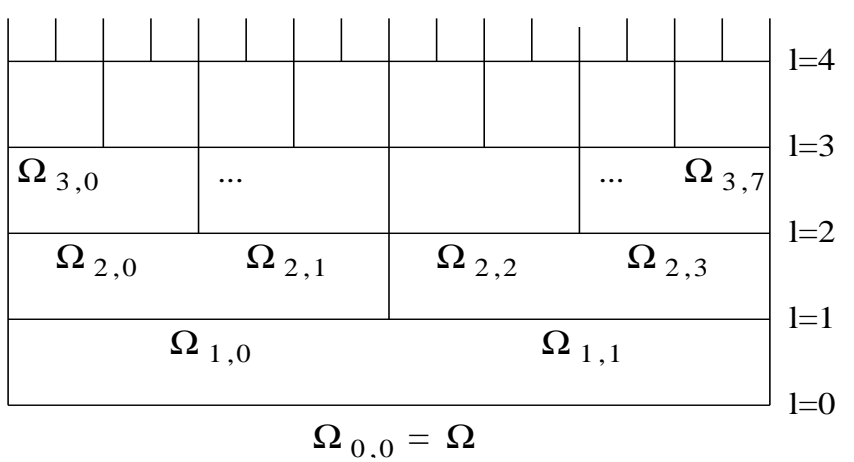

FiguRE 13. Hierarchy of partitions of $\Omega$. 
The dyadic grids $X^{j}$ form a hierarchy of partitions of the interval $[0,1]=\cup_{k} \bar{\Omega}_{j, k}$ by disjoint cells $\Omega_{j, k}=$ $\left(k 2^{-j},(k+1) 2^{-j}\right), k=0, \cdots, 2^{j}-1$, as illustrated in Figure 13. Consider now the setting where the discretization operator $\mathcal{D}_{j}: f \rightarrow \mathbf{f}_{j}$ is defined for absolutely integrable functions $f$ in $[0,1]$ by the cell averages on the partition at level $j$

$$
f_{j, k}=2^{j} \int_{\Omega_{j, k}} f(x) d x
$$

Since each cell $\Omega_{j, k}$ is formed by two children cells, of equal size, at level $j+1$, that is,

$$
\bar{\Omega}_{j, k}=\bar{\Omega}_{j+1,2 k} \cup \bar{\Omega}_{j+1,2 k+1},
$$

it follows that the restriction operation $P_{j+1 \rightarrow j}: \mathbf{f}_{j+1} \rightarrow \mathbf{f}_{j}$, that gives the exact cell averages at level $j$ from the corresponding values at level $j+1$, is defined by

$$
f_{j, k}=\frac{1}{2}\left[f_{j+1,2 k}+f_{j+1,2 k+1}\right]
$$

\section{Cell-averages predictions}

Now the purpose of the prediction operation $P_{j \rightarrow j+1}: \mathbf{f}_{j} \rightarrow \tilde{\mathbf{f}}_{j+1}$ is to estimate the cell averages at level $j+1$ from the knowledge of the corresponding values at the coarser level $j$. The simplest way for predicting cell averages is by constant approximation, using the cell average of the mother cell $\Omega_{j, k}$ to predict the cell averages of its children cells $\Omega_{j+1,2 k}$ and $\Omega_{j+1,2 k+1}$

$$
\tilde{f}_{j+1,2 k}=\tilde{f}_{j+1,2 k+1}=f_{j, k},
$$

which is exact for constant functions. If we want exact reproduction of higher order polynomials, in addition to the cell average of the mother cell $\Omega_{j, k}$, we must also use cell averages of some neighbour cells. For instance, by including the cell averages of the two closest neighbours $\Omega_{j, k+1}$ and $\Omega_{j, k-1}$, for $1 \leq k \leq 2^{j}-2$, the predicted values

$$
\begin{aligned}
\tilde{f}_{j+1,2 k+1} & =f_{j, k}+\frac{1}{8}\left(f_{j, k+1}-f_{j, k-1}\right), \\
\tilde{f}_{j+1,2 k} & =f_{j, k}-\frac{1}{8}\left(f_{j, k+1}-f_{j, k-1}\right)
\end{aligned}
$$

result to be exact for quadratic polynomials. The restriction and prediction operations are illustrated in Figure 14 .

\section{Restriction Prediction}

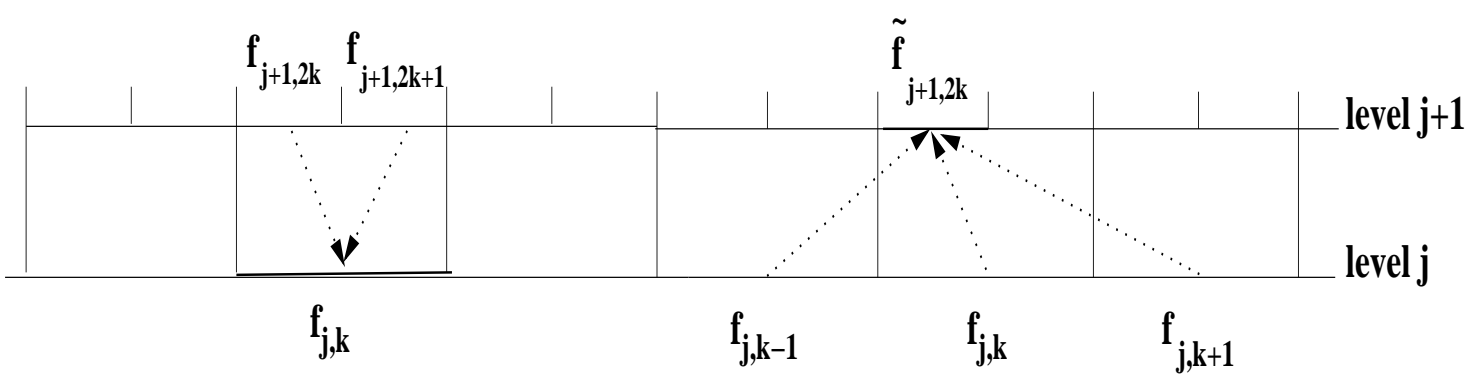

FIGURE 14. Illustration of the restriction and prediction operators. 
Similarly to the boundary treatment in the point value case, on the first cell of the left side we have

$$
\begin{aligned}
& \tilde{f}_{j+1,1}=\frac{11}{8} f_{j, 0}-\frac{1}{2} f_{j, 1}+\frac{1}{8} f_{j, 2}, \\
& \tilde{f}_{j+1,0}=\frac{5}{8} f_{j, 0}+\frac{1}{2} f_{j, 1}-\frac{1}{8} f_{j, 2},
\end{aligned}
$$

and on the right side

$$
\begin{aligned}
& \tilde{f}_{j+1,2^{j+1}-1}=-\frac{1}{8} f_{j, 2^{j}-3}+\frac{1}{2} f_{j, 2^{j}-2}+\frac{5}{8} f_{j, 2^{j}-1}, \\
& \tilde{f}_{j+1,2^{j+1}-2}=\frac{1}{8} f_{j, 2^{j}-3}-\frac{1}{2} f_{j, 2^{j}-2}+\frac{11}{8} f_{j, 2^{j}-1} .
\end{aligned}
$$

It can be verified that this prediction operator is derived from cell average polynomial interpolation in the sense that

$$
\begin{aligned}
\tilde{f}_{j+1,2 k+1} & =2^{j+1} \int_{\Omega_{j+1,2 k+1}} p(x) d x \\
\tilde{f}_{j+1,2 k} & =2^{j+1} \int_{\Omega_{j+1,2 k}} p(x) d x
\end{aligned}
$$

where $p(x)$ is the quadratic polynomial whose cell averages on the three closest stencil cells $\Omega_{j, m}$ with $|m-k| \leq 1$ coincide with $f_{j, m}$

$$
f_{j, m}=2^{j} \int_{\Omega_{j, m}} p(x) d x .
$$

This procedure may be extended to derive higher order predictions, using larger stencils which include $\Omega_{j, k}$ and $2 L$ closest neighbour cells $\Omega_{j, m}$ with $|m-k|<L, L>1$. Firstly, define $p(x)$ as the polynomial of degree $2 L$ whose cell averages on the $2 L$ stencil cells $\Omega_{j, m}$ coincide with $f_{j, m}$, and then define $\tilde{f}_{j+1,2 k}$ and $\tilde{f}_{j+1,2 k+1}$, as the cell average of $p(x)$ on the children cells $\Omega_{j+1,2 k}$ and $\Omega_{j+1,2 k+1}$. It is clear that such procedure will reproduce polynomials of degree $M=2 L$.

\section{MR transformations}

As in the case of discretization by point values, to construct multiresolution analyses for cell averages we may keep the details between a scale level $j$ and the next upper level $j+1$ as the prediction error at one of the children cells. For instance,

$$
d_{j, k}=f_{j+1,2 k+1}-\tilde{f}_{j+1,2 k+1} .
$$

Given $\mathbf{f}_{j}$ and $\mathbf{d}_{j}, \mathbf{f}_{j+1}$ may be recovered, as indicated in the next algorithms.

Analysis $-\mathbf{f}_{J} \rightarrow \mathbf{f}_{J}^{M R}=\left(\mathbf{f}_{0}, \mathbf{d}_{0}, \ldots, \mathbf{d}_{J-1}\right)$

- For $j=J-1, \ldots, 0$, given $\mathbf{f}_{j+1}$ :

Do restriction: $P_{j+1 \rightarrow j}: \mathbf{f}_{j+1} \underset{\mathbf{f}_{j}}{\rightarrow}$

Do prediction: $P_{j \rightarrow j+1}: \mathbf{f}_{j} \rightarrow \tilde{\mathbf{f}}_{j+1}$

Compute prediction errors: $d_{j, k}=f_{j+1,2 k+1}-\tilde{f}_{j+1,2 k+1}$

Synthesis - $\mathbf{f}_{J}^{M R}=\left(\mathbf{f}_{0}, \mathbf{d}_{0}, \ldots, \mathbf{d}_{J-1}\right) \rightarrow \mathbf{f}_{J}$

- For $j=0, \ldots, J-1$, given $\mathbf{f}_{j}, \mathbf{d}_{j}$ :

Do prediction: $P_{j \rightarrow j+1}: \mathbf{f}_{j} \rightarrow \tilde{\mathbf{f}}_{j+1}$ 
Compute $\mathbf{f}_{j+1}$ :

$$
\begin{aligned}
f_{j+1,2 k+1} & =d_{j, k}+\tilde{f}_{j+1,2 k+1} \\
f_{j+1,2 k} & =2 f_{j, k}-f_{j+1,2 k+1}
\end{aligned}
$$

\section{Functional context}
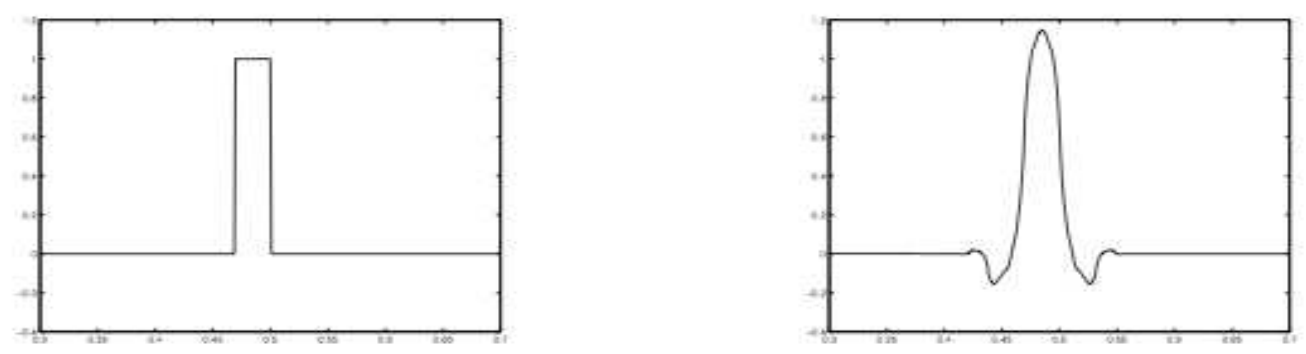

FIGURE 15. Cell-average scaling functions for constant (left side) and quadratic predictions (right side), at level $j=5$.
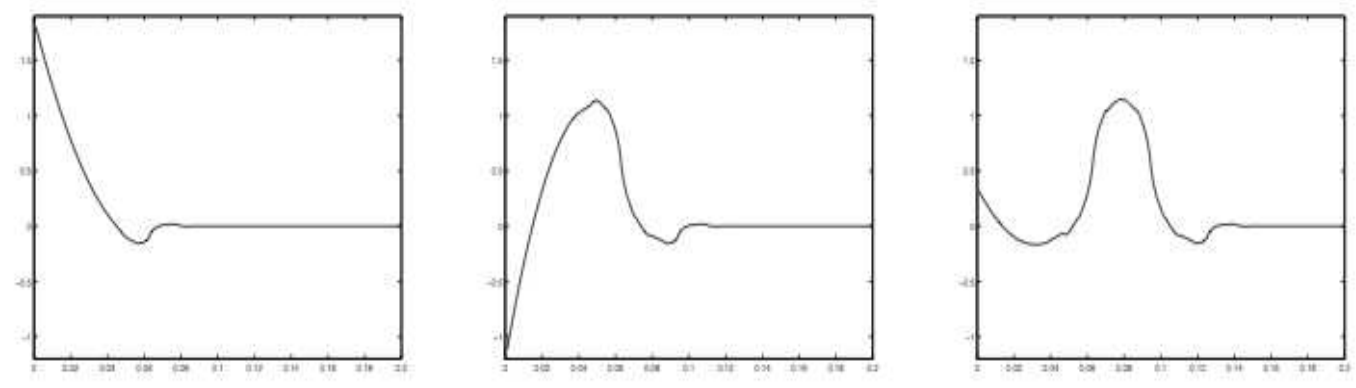

Figure 16. Cell-average scaling functions at level $j=5$, interacting with the left boundary, for quadratic predictions.

In the present case of cell average discretization, we can also define multiresolution decompositions like (5-6). It is clear that

$$
f_{j, k}=2^{j} \int_{\Omega_{j, k}} f(x) d x=<f, \Phi_{j, k}^{*}>,
$$

where $\Phi_{j, k}^{*}=2^{j} \chi_{\Omega_{j, k}}$. Here $\chi_{C}$ is the characteristic function of the set $C$, whose values are 1 for $x \in C$ and 0 otherwise. The scaling relation holds

$$
\Phi_{j, k}^{*}=\frac{1}{2}\left[\Phi_{j+1,2 k}^{*}+\Phi_{j+1,2 k+1}^{*}\right]
$$

It also follows that the dual wavelets $\Psi_{j, k}^{*}$ such that

$$
d_{j, k}=f_{j+1,2 k+1}-\tilde{f}_{j+1,2 k+1}=<f, \Psi_{j, k}^{*}>
$$


are given by

$$
\begin{aligned}
\Psi_{j, k}^{*} & =2^{j+1} \chi_{\Omega_{j+1,2 k+1}}-2^{j} \sum_{m} h_{m}^{k} \chi_{\Omega_{j, m}} \\
& =\Phi_{j+1,2 k+1}^{*}-\sum_{m} h_{m}^{k} \Phi_{j, m}^{*}
\end{aligned}
$$

where $h_{m}^{k}$ are the prediction coefficients, such that

$$
\tilde{f}_{j+1,2 k+1}=\sum_{m} h_{m}^{k} f_{j, m} .
$$

The definition of the primal basic functions $\Phi_{j, k}$ and $\Psi_{j, k}$ relies on the convergence of the prediction operator. A cell-average prediction is convergent if for any starting sequence of $s_{j}=\left(s_{j, k}\right)$, there exists an integrable function $s_{j}(x)$ such that its cell averages at levels $\ell \geq j$ coincide with the output of the iterative cell-average subdivision $s_{\ell}=P_{\ell-1 \rightarrow \ell} s_{\ell-1}$. If this property holds, average-interpolating scaling functions $\Phi_{j, k}(x)$ may be defined by cell-average subdivision of delta sequences $s_{j, m}=\delta(k-m)$. In the functional spaces

$$
V_{j}=\operatorname{span}\left\{\Phi_{j, k}(x), k=0, \cdots, 2^{j}-1\right\}
$$

the expansion

$$
f_{j}(x)=\sum_{k=0}^{2^{j}-1} f_{j, k} \Phi_{j, k}(x) \in V_{j}
$$

corresponds to the cell-average reconstruction of an integrable function $f$, in the sense that $\mathcal{D}_{j} f_{j}(x)=\mathbf{f}_{j}$, i.e., the cell averages of $f$ and $\mathbf{f}_{j}$ coincide at level $j$.

Using the same arguments of the interpolating setting, we obtain scaling relations

$$
\Phi_{j, k}(x)=\sum_{m} c_{k}(m) \Phi_{j+1, m}(x),
$$

where $c_{k}(m)$ are the cell averages of $\Phi_{j, k}$ at level $j+1$, which are obtained from the prediction of the deltasequence $\delta(k-m)$. The following properties hold for these cell-average scaling functions [36]

- For $N>0, \Phi_{j, k}$ is uniformly continuous, with Hölder regularity $s=s(N)$ increasing with interpolation degree $N$. For instance, $\Phi_{j, k}(x) \in C^{s}$ with $s>0.678$ and $s>1.272$ for $N=2$ and 4 , respectively (in fact, this is a consequence of a commutation formula expressing the derivative of interpolating scaling functions using polynomial interpolation of degree $M-1$ in terms of divided differences of cell average scaling functions for $N=M-2$ ).

- Far from the boundaries, $\Phi_{j, k}(x)=\phi\left(2^{j} x-k\right)$, where $\phi=\varphi_{1, N+1}$ are the Cohen-Daubechies-Feauveau [15] spline scaling functions.

- They have compact support, with size $\left|\operatorname{supp}\left(\Phi_{j, k}\right)\right|=O\left(2^{-j}\right)$, increasing with $M$.

Figure 15 displays the scaling functions $\Phi_{j, k}(x)$ for constant and quadratic cell-average interpolations, at level $j=5$, at the center of the interval. The scaling functions interacting with the left boundary, for quadratic prediction, are shown in Figure 16.

Having that $V_{j} \subset V_{j+1}$, we now examine complementary spaces $W_{j}$ such that $V_{j+1}=V_{j}+W_{j}$, and wavelets $\Psi_{j, k}$ that span these differences. As such, the multiresolution expansion of $\Psi_{j, k}$ is expected to correspond to zero cell averages at level $j$, and wavelet coefficients $d_{j, m}=\delta(k-m)$. Therefore, it should be given by the expression

$$
\Psi_{j, k}(x)=\Phi_{j+1,2 k+1}(x)-\Phi_{j+1,2 k}(x) .
$$

Figure 17 shows the plots for the Haar wavelet $(N=0)$ and for quadratic prediction. For the particular case 

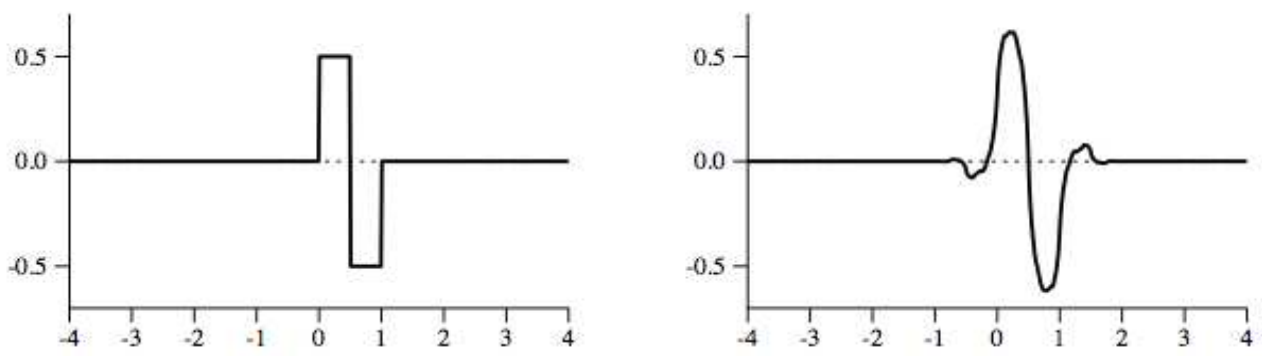

Figure 17. Haar wavelet (left side) and wavelet for quadratic predictions (right side).

of prediction by constant approximation, $\Phi_{j, k}=\chi_{\Omega_{j, k}}$ and the spaces $V_{j}$ are formed by piecewise constant functions, generate a multiresolution analysis of $L^{2}(\Omega)$ such that $V^{j} \subset V^{j+1}, \cup_{j \geq 0} V^{j}$ is dense in $L^{2}(\Omega)$ and $\left\{\Phi_{j, k}, k=0, \cdots, 2^{j}-1\right\}$ is an orthogonal basis for $V^{j}$, and the expansion (8) corresponds to the orthogonal projection of $f$ on $V^{j}$. The wavelet spaces $W^{j}=\operatorname{span}\left\{\Psi_{j, k}, k=0, \cdots, 2^{j}-1\right\}$ are the orthogonal complement of $V_{j}$ in $V_{j+1}$, and $\Psi_{j, k}(x)$ are the known Haar wavelets.

For general cell-average predictions by polynomials of higher degree $M=2 L$, the spaces $V_{j}$ also generate a multiresolution analysis of $L^{1}(\Omega)$, such that $V^{j} \subset V^{j+1}, \cup_{j \geq 0} V^{j}$ is dense in $L^{1}[0,1]$. The sequence of approximations $f_{j}(x)$ corresponds to the biorthogonal projections of $f$ on $V^{j}$ and

$$
\begin{aligned}
\left\|f_{j}\right\|_{L^{1}} & \leq C\|f\|_{L^{1}} \\
\left\|f-f_{j}\right\|_{L^{1}} & \rightarrow 0, \text { as } j \rightarrow \infty .
\end{aligned}
$$

For a comprehensive reference on the convergence properties of MR approximations, we refer to [14].

\section{Local Regularity Wavelet Indicator}

In the cell-average context, the wavelet coefficients can also be used as local regularity indicators. Assume that the function $f(x)$ has $C^{s}$-smoothness, for some $s \leq M$, within the interval $I_{j, k}$ supporting $\Psi_{j, k}^{*}$ (i.e. $I_{j, k}$ contains the stencil cells used in the prediction of $\left.\tilde{f}_{j+1,2 k+1}\right)$. Using classical results of local polynomial approximation by polynomials, and the polynomial cancellation property, the wavelet coefficient can be estimated by

$$
\begin{aligned}
\left|d_{j, k}\right| & \leq \inf _{q \in \Pi_{M-1}}\|f-q\|_{L^{\infty}\left(I_{j, k}\right)} \| \Psi_{j, k}^{*}||_{L^{1}} \\
& \leq C 2^{-s j}|f|_{C^{s}\left(I_{j, k}\right)}
\end{aligned}
$$

where the facts $\left\|\Psi_{j, k}^{*}\right\|=1$, and that the size o $I_{j, k}$ is $O\left(2^{-j}\right)$ have been used. Therefore, for high accurate predictions, the fast decay of wavelet coefficients is ensured in smooth regions.

\section{Data Compression}

Given the cell averages $\mathbf{f}_{J}$ on the finest level $J$, after the iteration of the MR transform, they can be represented as

$$
\mathbf{f}_{J}^{M R}=\left(\mathbf{f}_{0}, \mathbf{d}_{0}, \cdots, \mathbf{d}_{J-1}\right)
$$

Data compression is obtained after a truncation step

$$
d_{j, k}^{\epsilon}=\left\{\begin{array}{cc}
0 & \text { if }\left|d_{j, k}\right| \leq \epsilon_{j} \\
d_{j, k} & \text { otherwise. }
\end{array}\right.
$$


Figure 18 shows the plot of functions $f(x)$ on the top side, having different regularity patterns. The position of their significant wavelet coefficients, given bellow, reflects the different decay of the coefficients, and thus illustrates their local regularity characterization.
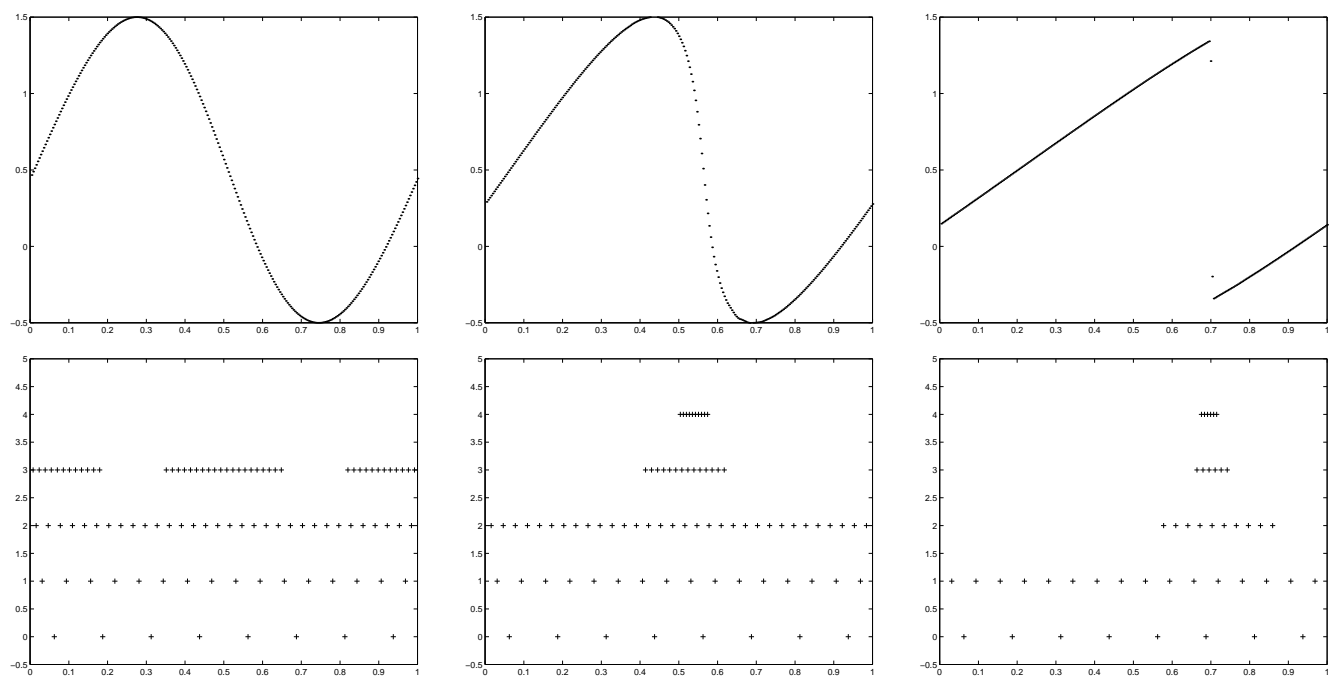

Figure 18. Thresholding operation. Top: function $f(x, t)$ at three different time instants exhibiting a steepening slope. Bottom: corresponding retained wavelet coefficients after thresholding.

For the applications described in Section 5, in the context of finite volume methods, the main idea is to use wavelet coefficients in a multiresolution representation of the cell averages of the solution to construct adaptive grids: use coarser cells in regions where the wavelet coefficients are small (and the solution is smooth) and refine where they are significant.

\section{Stability}

We ask once again the same stability question: after applying the inverse MR transform to the compressed data $\mathbf{f}_{J}^{M R, \epsilon}$, how do the reconstructed values $\mathbf{f}_{J}^{\epsilon}$ compare to the original ones? Could the thresholding perturbation be amplified by iterative application of the cell-average prediction?

For convergent subdivision schemes we may use the expansion

$$
f_{J}(x)-f_{J}^{\epsilon}(x)=\sum_{j=0}^{J-1} \sum_{k}\left(d_{j, k}-d_{j, k}^{\epsilon}\right) \Psi_{j, k}(x) .
$$

Since the support of $\Psi_{j, k}$ is uniformly localized around $C_{j, k}$, such that $\left\|\Psi_{j, k}\right\|_{L^{1}} \leq C 2^{-j}$, the $L^{1}$ thresholding error at level $j$ is

$$
\begin{aligned}
\left|\int_{0}^{1} \sum_{k=0}^{2^{j}-1}\left(d_{j, k}-d_{j, k}^{\epsilon}\right) \Psi_{j, k}(x) d x\right| & \leq \epsilon_{j} \sum_{k=0}^{2^{j}-1}\left\|\Psi_{j, k}\right\|_{L^{1}} . \\
& \leq C \epsilon_{j} .
\end{aligned}
$$




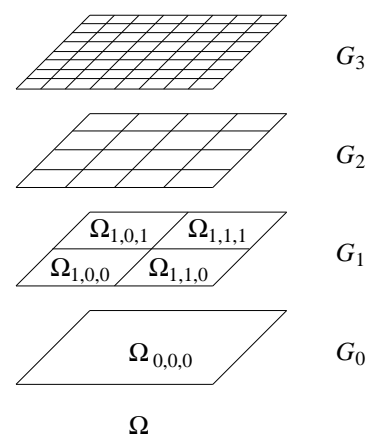

FIGURE 19. Hierarchy of nested meshes.

Consequently, the $L^{1}$-stability of thresholding error overall the scale levels is estimated by

$$
\left\|f_{J}-f_{J}^{\epsilon}\right\|_{L^{1}} \leq C \sum_{j=0}^{J-1} \epsilon_{j} .
$$

If $\epsilon_{J-1}=\epsilon$, and $\epsilon_{j}=\frac{1}{2} \epsilon_{j+1}=\epsilon 2^{j-J}$, we conclude that the thresholding error is uniformly bounded by $C \epsilon$, up to a change in the constant $C$.

\section{Cartesian Grids in Higher Dimensions}

In higher dimensions, multiresolution schemes for Cartesian grids can be obtained by tensor products of 1D schemes. For instance, in 2D geometry, we consider a hierarchy of Cartesian meshes

$$
G_{j}=\left\{\Omega_{j, \gamma}\right\}, \quad \gamma=(k, m) \in S_{j}, \quad \Omega=\cup_{\gamma \in S_{j}} \bar{\Omega}_{j, \gamma}, \quad\left|\Omega_{j, \gamma}\right| \sim 2^{-2 j}
$$

where $\Omega_{j, \gamma} \in G_{j}$ is the union of four cells in $G_{j+1}$. An illustration is given in Figure 19 .

For instance, for the prediction to be exact for quadratic polynomials, away from the boundaries, the neighbour cells $\Omega_{j,(k-1, m)}, \Omega_{j,(k+1, m)}, \Omega_{j,(k, m-1)}$ and $\Omega_{j,(k, m+1)}$ are considered, and for $n, p \in\{0,1\}$, the prediction formula reads $[10]$

$$
\begin{aligned}
\tilde{f}_{j+1,(2 k+n, 2 m+p)} & =f_{j,(k, m)}+\frac{1}{8}(-1)^{p}\left(f_{j,(k, m+1)}-f_{j,(k, m-1)}\right)+\frac{1}{8}(-1)^{n}\left(f_{j,(k+1, m)}-f_{j,(k-1, m)}\right) \\
& +\frac{1}{64}(-1)^{n p}\left[\left(f_{j,(k+1, m+1)}-f_{j,(k+1, m-1)}\right)-\left(f_{j,(k-1, m+1)}-f_{j,(k-1, m-1)}\right) .\right.
\end{aligned}
$$

Now, three wavelet coefficients have to be considered and the two-level transformations are $\mathbf{f}_{j+1} \leftrightarrow\left\{\mathbf{f}_{j}, \mathbf{d}_{j}^{(1)}, \mathbf{d}_{j}^{(2)}, \mathbf{d}_{j}^{(3)}\right\}$, where

$$
\begin{aligned}
& d_{j,(k, m)}^{(1)}=f_{j+1,(2 k, 2 m+1)}-\tilde{f}_{j+1,(2 k, 2 m+1)} \\
& d_{j,(k, m)}^{(2)}=f_{j+1,(2 k+1,2 m)}-\tilde{f}_{j+1,(2 k+1,2 m)} \\
& d_{j,(k, m)}^{(3)}=f_{j+1,(2 k+1,2 m+1)}-\tilde{f}_{j+1,(2 k+1,2 m+1)} .
\end{aligned}
$$



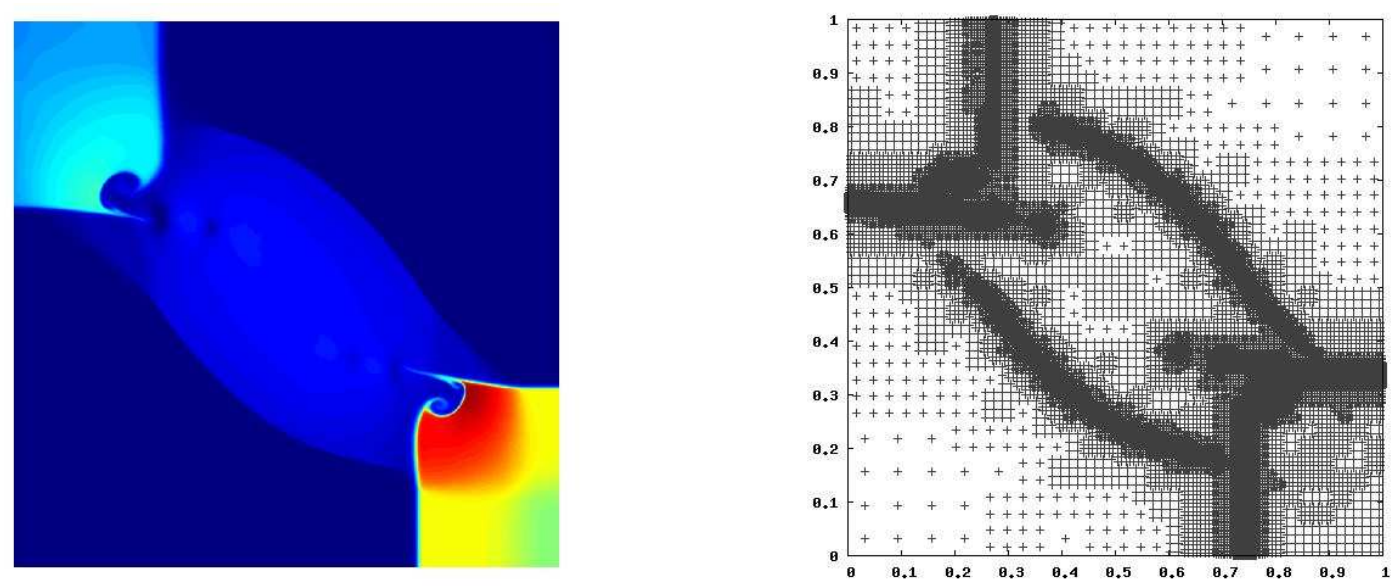

FiguRE 20. Example of an adaptive grid (right) obtained by thresholding wavelets coefficients of the density field (left) (from [27]).

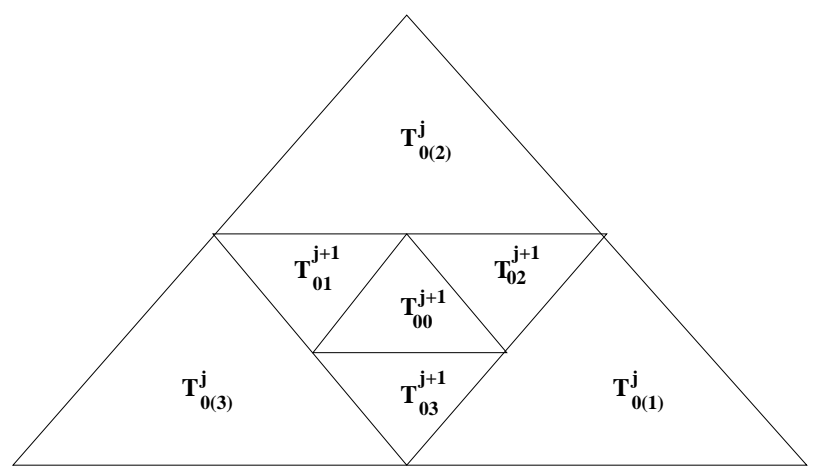

Figure 21. Triangle $T_{0}^{j}$, the four children $T_{\mu}^{j+1}, \mu=(0, i)$, and neighbours $T_{0, i}^{j}, i=0,1,2,3$.

Using this third order MR scheme for cell averages, Figure 20 shows an adaptive grid for the numerical solution of a Riemann problem, where the cells are chosen according to those wavelet coefficients with magnitude above a given threshold. For details we refer to [27].

\section{General Geometries}

Harten's approach can be extended to discretizations based on general geometries, as proposed in [1]. For instance, consider a hierarchy of triangular meshes as indicated in Figure 21

$$
G_{j}=\left\{T_{\gamma}^{j}\right\}, \Omega=\cup_{\gamma \in S_{j}} \bar{T}_{\mu}^{j}, \quad\left|T_{\gamma}^{j}\right| \sim 2^{-2 j},
$$

such that $T_{\gamma}^{j}$ is the union of four children triangles $\mathcal{C}_{\gamma}^{j}=\left\{T_{\mu}^{j+1}, \mu=(\gamma, i), i=0,1,2,3\right\}$ at level $j+1$

$$
\bar{T}_{\gamma}^{j}=\bigcup_{\mu \in \mathcal{C}_{\gamma}^{j}} \bar{T}_{\mu}^{j+1}
$$




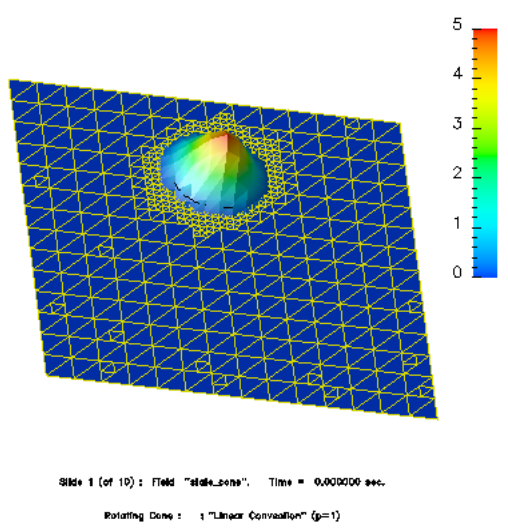

FIGURE 22. Adaptive triangular grid indicated by significant Haar wavelet coefficients.

Given the cell averages $\mathbf{f}_{j}=\left(f_{j, \gamma}\right)$, where

$$
f_{j, \gamma}=\frac{1}{\left|T_{\gamma}^{j}\right|} \int_{T_{\gamma}^{j}} u(\mathbf{x}) d \mathbf{x}
$$

the restriction operator $P_{j+1 \rightarrow j}: \mathbf{f}_{j+1} \rightarrow \mathbf{f}_{j}$ is straightforward

$$
f_{j, \gamma}=\frac{1}{\left|T_{\gamma}^{j}\right|} \sum_{\mu \in \mathcal{C}_{\gamma}^{j}}\left|T_{\mu}^{j+1}\right| f_{j+1, \mu}
$$

For the definition of the prediction operator $P_{j \rightarrow j+1}: \mathbf{f}_{j} \rightarrow \tilde{\mathbf{f}}_{j+1}$, the easiest way is by piecewise constant approximation

$$
\tilde{\mathbf{f}}_{j+1, \mu}=f_{j, \gamma}, \quad \mu \in \Omega_{\gamma}^{j}
$$

which gives the three Haar wavelet coefficients

$$
d_{j, \gamma}^{(i)}=f_{j+1, \mu}-\tilde{f}_{j+1, \mu}, \mu=(\gamma, i) \in \mathcal{C}_{\gamma}^{j}, i=1,2,3
$$

as indicated in Figure 23. Since Haar wavelets cancel for constant polynomials $q \in \Pi_{0}$, it follows that

$$
\begin{aligned}
\left|d_{j, \gamma}^{(i)}\right| & \leq \inf _{q \in \Pi_{0}}\|\mathbf{u}-q\|_{L^{\infty}\left(\bar{T}_{j, \gamma}\right)} \\
& \leq C 2^{-j}\|\mathbf{u}\|_{C^{1}\left(\bar{T}_{j, \gamma}\right)}
\end{aligned}
$$

which is the basis for the use of such coefficients as local regularity indicators. Figure 22 shows an adaptive triangular grid, where the cells correspond to significant Haar wavelet coefficients. Examples of applications of Haar wavelets on triangles are described in [11] for the definition of adaptive strategies for the discontinuous Galerkin method. 


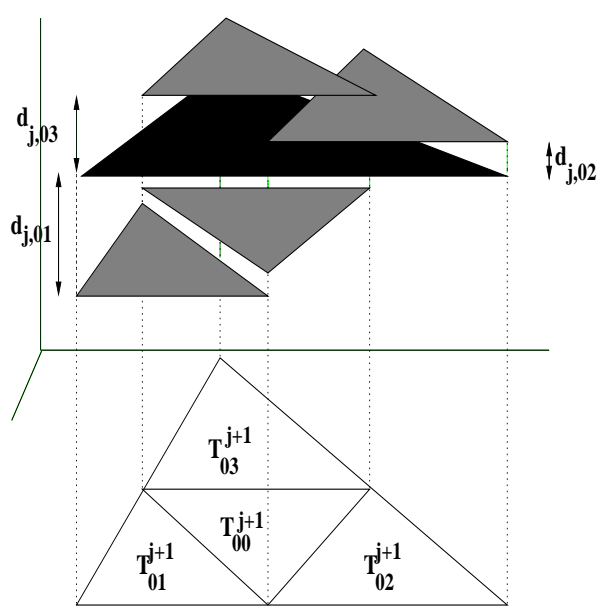

FiguRE 23. Haar wavelet coefficients on triangles.

For more accurate predictions, the suggestion in [16] is

$$
\tilde{f}_{j+1, \mu}= \begin{cases}f_{j, \gamma} & \mu=(\gamma, 0) \\ f_{j, \gamma}+\frac{1}{6}\left[f_{j, \gamma(2)}-f_{j, \gamma(1)}\right]+\frac{1}{6}\left[f_{j, \gamma(3)}-f_{j, \gamma(1)}\right] & \mu=(\gamma, 1) \\ f_{j, \gamma}+\frac{1}{6}\left[f_{j, \gamma(1)}-f_{j, \gamma(2)}\right]+\frac{1}{6}\left[f_{j, \gamma(3)}-f_{j, \gamma(2)}\right] & \mu=(\gamma, 2) \\ f_{j, \gamma}+\frac{1}{6}\left[f_{j, \gamma(1)}-f_{j, \gamma(3)}\right]+\frac{1}{6}\left[f_{j, \gamma(2)}-f_{j, \gamma(3)}\right] & \mu=(\gamma, 3)\end{cases}
$$

which holds for equilateral triangular partitions, where the triangles $T_{\gamma(i)}^{j}, i=1,2,3$ denote the three neighbours of $T_{\gamma}^{j}$. This prediction results to be convergent, and exact for first order polynomials. For applications of this scheme to the adaptive solution of conservation laws we refer to $[17,18]$.

\subsection{The Lifting Methodology}

The basic idea in the lifting methodology is to start from a simple framework, Haar-like wavelets for instance, and to use the lifting scheme (or dual lifting) [77] to obtain a new multiresolution analysis with better performance. The block diagram of Figure 24 illustrates the lifting procedure in terms of two operations: prediction $\mathcal{P}$ and update $\mathcal{U}$. Suppose that the input data $\mathbf{f}_{j+1}$ is subjected to a given MR scheme to produce coarse values $\mathbf{f}_{j}$ and details $\mathbf{d}_{j}$. The prediction acts on the coarse data to modify the detail coefficients
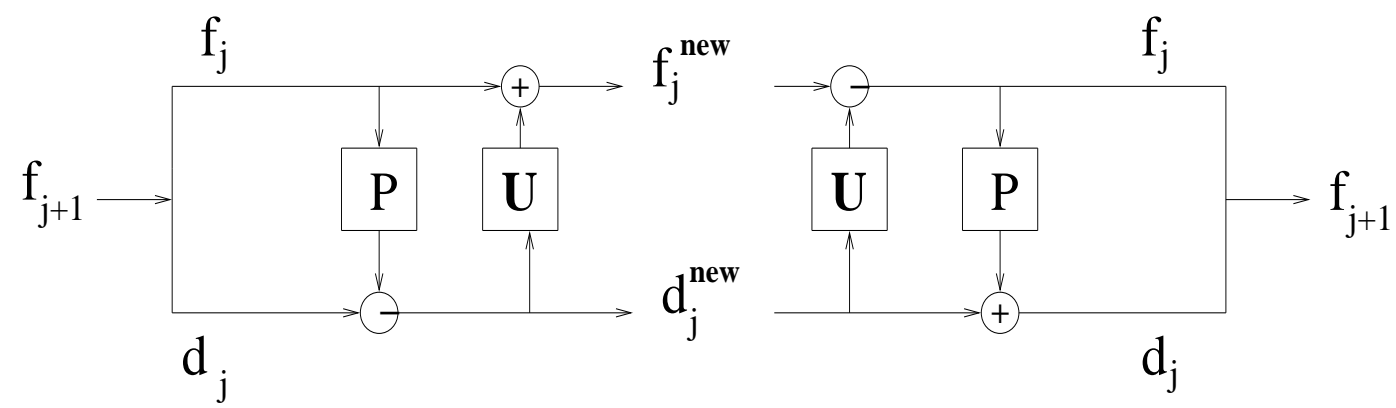

Figure 24. The flowchart of lifting scheme. 


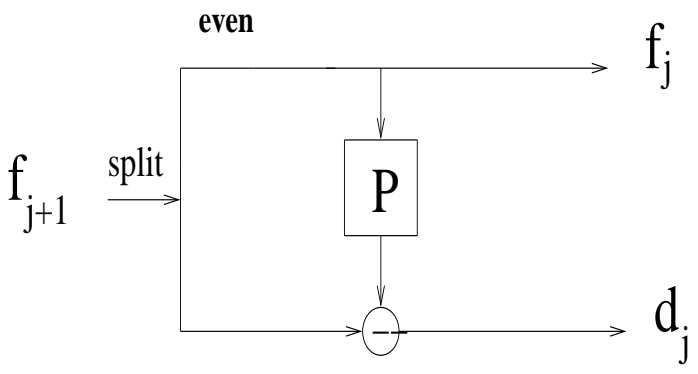

odd

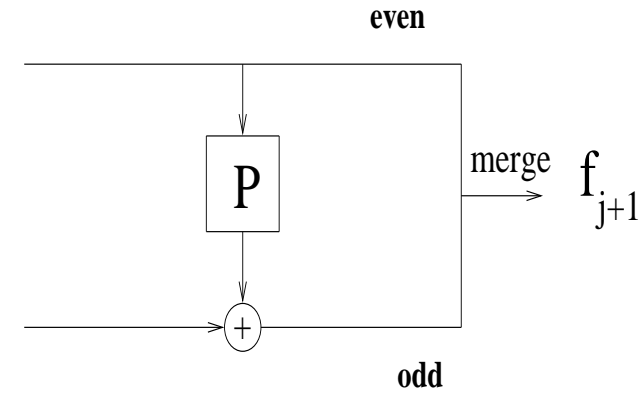

FIGURE 25. Interpolating $\mathrm{MR}=$ lifting the lazy $\mathrm{MR}$.

$$
\mathbf{d}_{j}^{\text {new }}=\mathbf{d}_{j}-\mathcal{P} \mathbf{f}_{j} .
$$

Then, the operation $\mathcal{U}$ is applied to the details in order to modify the coarse data

$$
\mathbf{f}_{j}^{\text {new }}=\mathbf{f}_{j}+\mathcal{U} \mathbf{d}_{j}^{\text {new }}
$$

Once the forward lifting transform is defined, its inverse can be easily constructed by reversing the operations. Given the input $\left\{\mathbf{f}_{j}^{\text {new }}, \mathbf{d}_{j}^{\text {new }}\right\}$, first recover $\left\{\mathbf{f}_{j}, \mathbf{d}_{j}\right\}$

$$
\begin{aligned}
\mathbf{f}_{j} & =\mathbf{f}_{j}^{\text {new }}-\mathcal{U} \mathbf{d}_{j}^{\text {new }} \\
\mathbf{d}_{j} & =\mathbf{d}_{j}^{\text {new }}+\mathcal{P} \mathbf{f}_{j}
\end{aligned}
$$

and then apply the original inverse MR transform to get $\mathbf{f}_{j+1}$.

Lifting provides an efficient methodology for custom-design construction of wavelets on arbitrary topological domains, e.g. for wavelets on the sphere [74]. To give a basic idea of this methodology, we describe some examples to show how MR transforms for point values and cell averages can be formulated using lifting tools, starting from very simple schemes.

\subsubsection{Lifting the Interpolating Wavelets}

Multiresolution for point values can be formulated in the lifting context. Consider the simplest MR scheme (lazy MR) where the analysis step just splits the data in two sets of even and odd indexes

$$
\begin{array}{ll}
\left(\mathbf{e v e n}_{j}, \operatorname{odd}_{j}\right):= & \operatorname{Split}\left(\mathbf{f}_{j+1}\right) \\
f_{j, k}=f_{j+1,2 k}, & d_{j, k}=f_{j+1,2 k+1},
\end{array}
$$

and the synthesis corresponds to merging operation

$$
\begin{aligned}
\mathbf{f}_{j+1}:= & \operatorname{Merge}\left(\text { even }_{j}, \operatorname{odd}_{j}\right) \\
f_{j+1,2 k}=f_{j, k}, & f_{j+1,2 k+1}=d_{j, k} .
\end{aligned}
$$

If an interpolating prediction $\mathcal{P}=P_{j \rightarrow j+1}$ is introduced, the lazy wavelet coefficients are modified

$$
\begin{aligned}
d_{j, k}^{\text {new }} & =\operatorname{odd}_{j, k}-\mathcal{P}(\text { even })_{j, k} \\
& =f_{j+1,2 k+1}-\tilde{f}_{j+1,2 k+1},
\end{aligned}
$$

which coincides with the result given by Harten's approach. Figure 25 illustrates this new interpretation of the interpolating MR inter-level operations in the lifting context. 

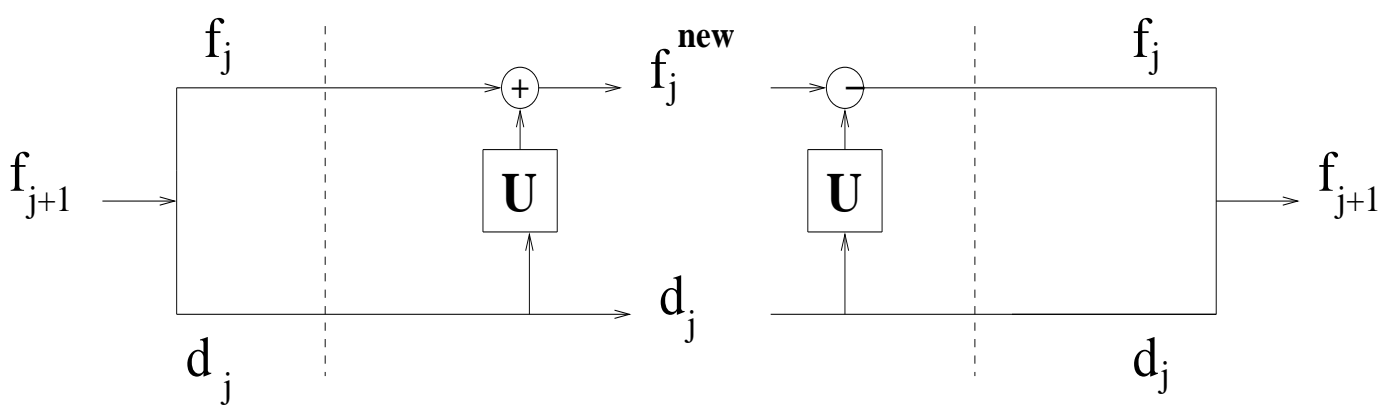

FIGURE 26. Flowchart illustrating the lifting of the MR scheme.

In interpolating MR contexts, one disadvantage is that the average of the information $\mathbf{f}_{j}$ is not conserved within the scale levels. Using the lifting methodology, an update operation may be defined to modify the scaling coefficients in order to preserve averages

$$
\frac{1}{2^{j}} \sum_{k} f_{j, k}^{n e w}=\frac{1}{2^{j+1}} \sum_{k} f_{j+1, k} .
$$

For instance, given the interpolating MR scheme where

$$
d_{j, k}=f_{j+1,2 k+1}-\frac{f_{j, k}+f_{j, k+1}}{2}
$$

we can define

$$
f_{j, k}^{n e w}=f_{j, k}+\frac{d_{j, k-1}+d_{j, k}}{4} .
$$

This procedure is illustrated in Figure 26. Since

$$
\sum_{k} d_{j, k}=\sum_{k} f_{j+1,2 k+1}-\sum_{k} f_{j+1,2 k}
$$

we obtain the desired property

$$
\begin{aligned}
\sum_{k} f_{j, k}^{n e w} & =\sum_{k} f_{j+1,2 k}+\frac{d_{j, k-1}+d_{j, k}}{4} \\
& =\sum_{k} f_{j+1,2 k}+\frac{1}{2} \sum_{k} f_{j+1,2 k+1}-\frac{1}{2} \sum_{k} f_{j+1,2 k} \\
& =\frac{1}{2} \sum_{k} f_{j+1, k} .
\end{aligned}
$$

In the functional context, the updating step does not affect the primal scaling functions, $\Phi_{j, k}^{n e w}(x)=\Phi_{j, k}(x)$, which means that the multiresolution spaces are not modified, $V_{j}^{\text {new }}=V_{j}$. Furthermore, since the wavelet coefficients are not modified, the dual wavelets $\Psi_{j, k}^{*}(x)$, such that $d_{j, k}=<f, \Psi_{j, k}^{*}>$, are also preserved. However, the modification of the scaling coefficients implies that new dual scaling functions $\Phi_{j, k}^{*, n e w}(x)$ such that

$$
f_{j, k}^{n e w}=<f, \Phi_{j, k}^{*, n e w}>
$$

and new primal wavelets $\Psi_{j, k}^{n e w}(x)$ appear. This means that the effect of updating is the creation of a new factorization of the scaling spaces

$$
V_{j+j}=V_{j}+W_{j}^{n e w}
$$




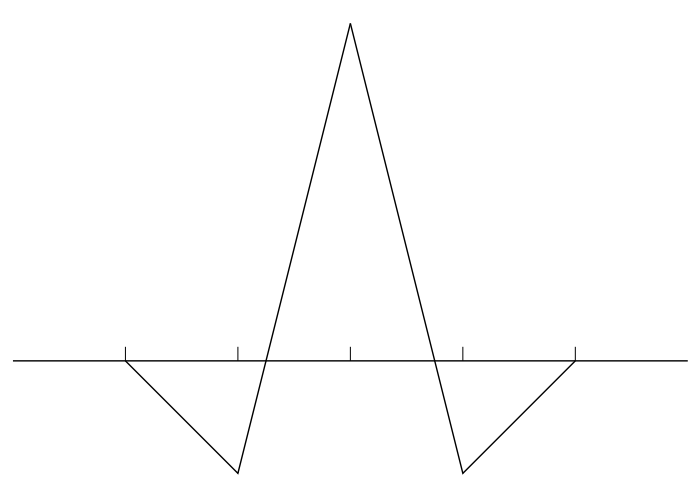

FIGURE 27. Updated primal wavelet $\Psi_{j, k}^{n e w}(x)$.

For instance, in example (9), we have

$$
\Phi_{j, k}^{*, n e w}(x)=\Phi_{j, k}^{*}(x)+\frac{1}{4}\left[\Psi_{j, k-1}^{*}(x)+\Psi_{j, k}^{*}(x)\right]
$$

The new primal wavelets $\Psi_{j, k}^{n e w}(x)$ are the functions in $V_{j+1}$ whose point values at $X_{j+1}$ are obtained by the updated synthesis of zero values in $X_{j}$ and wavelet coefficients $d_{j, m}=\delta(k-m)$, that is $\cdots, 0,-1 / 4,1,-1 / 4,0, \cdots$. Consequently, they are the continuous piecewise linear functions as shown in Figure 27.

\subsubsection{Dual Lifting the Haar Wavelets}

Suppose that for the applications of our interest, starting with cell average data, we are not interested in modifying the coarse values, since we want to keep the cell averages at all levels. Therefore, we skip the updating step and just modify the wavelet coefficients. If the original scheme is of Haar type, which is only consistent for constant functions, the purpose is to obtain a new MR scheme with higher order of consistency. This kind of procedure is known as dual lifting transform.

As described in Section 2.1.2, Haar wavelets have one vanishing moment only, i.e., the Haar wavelet coefficients vanish only for constant polynomials. The dual lifting scheme can be used to construct new MR settings connected to dual scaling functions $\left\{\Phi_{j, k}^{n e w}, \Phi_{j, k}^{* n e w}\right\}$ such that the corresponding wavelet coefficients vanish for higher degree polynomials. The basic idea is to keep the dual scaling functions $\Phi_{j, k}^{* n e w}=\Phi_{j, k}^{*}$, but to modify the other basic functions accordingly to the new requirements. This means that in the forward MR transform, the scaling coefficients $f_{j, k}=<f, \phi_{j, \alpha}^{*}>$ remain the same, but the Haar wavelet coefficients $d_{j, k}$ are updated using the scaling coefficients $f_{j, m}$ corresponding to neighbouring cells $\Omega_{j, m}, m \in \mathcal{S}(k)$

$$
d_{j, k}^{n e w}=d_{j, k}^{\text {Haar }}-\sum_{m \in \mathcal{S}(k)} c_{m}^{k} f_{j, m},
$$

which leads to the new dual wavelets

$$
\Psi_{j, k}^{* n e w}=\Psi_{j, k}^{*}-\sum_{m \in \mathcal{S}(k)} c_{m}^{k} \Phi_{j, m}^{*} .
$$

The coefficients $c_{m}^{k}$ are determined in order to cancel the desired polynomials $q$

$$
\sum_{m \in \mathcal{S}(k)} c_{m}^{k}<q, \Phi_{j, m}^{*}>=<q, \Psi_{j, k}^{*}>
$$


Since the scaling coefficients are not modified, the dual scaling functions are preserved, such that $f_{j, k}^{n e w}=<$ $f, \Phi_{j, k}^{*}>$. The modification of the wavelet coefficients implies new dual wavelets $\Psi_{j, k}^{*, n e w}(x)$ (dual lifting) such that

$$
d_{j, k}^{n e w}=<f, \Psi_{j, k}^{*, n e w}>
$$

new primal scaling functions $\Phi_{j, k}^{n e w}$, and new primal wavelets $\Psi_{j, k}^{n e w}$. Consequently, the inclusion of the prediction leads to new MR spaces

$$
V_{j+1}^{\text {new }}=V_{j}^{\text {new }}+W_{j}^{\text {new }} .
$$

For example, we consider the dual lifting of the Haar wavelets in order to cancel quadratic polynomials

$$
\begin{aligned}
d_{j, \gamma}^{\text {new }} & =d_{j, k}^{\text {Haar }}-\frac{1}{8}\left[f_{j, k+1}-f_{j, k-1}\right] \\
& =f_{j+1,2 k+1}-\left[f_{j, k}+\frac{1}{8}\left(f_{j, k+1}-f_{j, k-1}\right),\right]
\end{aligned}
$$

which coincides with the third order scheme derived in Section 2.1.2 using Harten's approach. Note also that the wavelet coefficients of second order MR scheme on equilateral triangles [16] can be expressed as

$$
d_{j, \mu}^{(i)}=d_{j, \mu}^{(i), \text { Haar }}-\left\{\begin{array}{cc}
\frac{1}{6}\left[f_{j, \gamma(2)}-f_{j, \gamma(1)}\right]+\frac{1}{6}\left[f_{j, \gamma(3)}-f_{j, \gamma(1)}\right] & \mu=(\gamma, 1) \\
\frac{1}{6}\left[f_{j, \gamma(1)}-f_{j, \gamma(2)}\right]+\frac{1}{6}\left[f_{j, \gamma(3)}-f_{j, \gamma(2)}\right] & \mu=(\gamma, 2) \\
\frac{1}{6}\left[f_{j, \gamma(1)}-f_{j, \gamma(3)}\right]+\frac{1}{6}\left[f_{j, \gamma(2)}-f_{j, \gamma(3)}\right] & \mu=(\gamma, 3) .
\end{array}\right.
$$

Therefore, they can also be interpreted in the context of dual lifting of the Haar wavelets. We refer to [21], where it is shown that the classical wavelet transforms, defined in terms of filter banks, can be decomposed into a finite sequence of lifting steps.

\subsection{Adaptation Concepts for Evolutionary PDEs}

We consider adaptive MR schemes for the numerical solution of evolutionary partial differential equations written in the form

$$
\frac{\partial u(x, t)}{\partial t}=\mathcal{D} u(x, t), t>0, x \in \Omega,
$$

where $\mathcal{D}$ is a differential operator in space. The above equation is completed with suitable initial and boundary conditions. The methods combine traditional accurate and stable schemes, such as finite volume or finite difference methods, with the ability of wavelet coefficients in the characterization of local regularity of functions. Using an appropriate MR framework, the idea is to represent the numerical solutions by the cell averages (or point values) corresponding to their significant wavelet coefficients. Typically, few information is required in each time step, the adaptive grids being coarse where the solution is smooth, and refined close to irregularities. At each cell (or point) of the adaptive grid the discretization of the spatial differential operator $\mathcal{D} u(x, t)$ is modified, using step size proportional to the local scale of each cell (or point).

Assume the reference numerical scheme in an explicit formulation

$$
U^{n+1}=\mathbf{E} U^{n}
$$

where the vectors $U^{n}=U_{J}^{n}$ contains the information of the numerical solution on the uniform grid at level $J$, and time $t^{n}, \mathbf{E}$ is the discrete evolution operator that includes the discretization of spatial derivatives, the action of a ODE solver and the enforcement of boundary conditions.

In the adaptive MR method, the goal is to use such reference scheme in a more efficient fashion by taking into account the wavelet local regularity information about the numerical solution. The first step in this direction is the representation of the numerical solution $U_{M R}^{n}$ on a sparse grid $\Gamma^{n}$. For the solution to evolve from $U_{M R}^{n}$ to $U_{M R}^{n+1}$, three basic stages are undertaken 
(1) Refinement: $U_{M R}^{n+} \leftarrow \mathbf{R} U_{M R}^{n}$

(2) Evolution: $\breve{U}_{M R}^{n+1} \leftarrow \mathbf{E}_{M R} U_{M R}^{n+}$

(3) Coarsening: $U_{M R}^{n+1} \leftarrow \mathcal{T}_{\epsilon} \breve{U}_{M R}^{n+1}$

The refinement operator $\mathbf{R}$ is a precaution measure to prevent sharp gradients to move into the coarse grid areas between time steps. Since the regions of smoothness or irregularities of the solution may change with time, $\Gamma^{n}$ may not be convenient any more at the next time step $t^{n+1}$. Therefore, before doing time evolution, the representation of the solution should be extended to a grid $\Gamma^{n+}$, which is expected to contain $\Gamma^{n+1}$. Then the evolution operator $\mathbf{E}_{M R}$ is applied. The subscript $M R$ indicates that the discretization of spatial derivatives is modified, using step size proportional to the local scale of each cell (or point). In the last stage, the thresholding operator is applied in order to coarsen some cells in $\Gamma^{n+}$ (or remove some points) where refinement is unnecessary for an accurate representation of $U_{M R}^{n+1}$.

For the implementation of this adaptive algorithm, the basic tools come from a multiresolution analysis for cell averages (or point values). As described previously, the discrete information of the solution is organized by levels or resolution. At each level $j$, wavelet coefficients $\mathbf{d}_{j}$ are defined as prediction error at the children cells (or new points) of the current level of discretization. Using the wavelet coefficients as local regularity indicators, the main ingredient is the thresholding operator $\mathcal{T}_{\epsilon}$ : only the information corresponding to the significant wavelets coefficients of magnitude above a threshold $\epsilon_{j}$ should be kept in the adaptive grid. The prediction operator is also used to calculate some required information that is not present in the adaptive computational grid. For instance, this is necessary in the refinement operation to approximate the solution at sites in $\Gamma^{n+} \backslash \Gamma^{n}$, and also in the application of $\mathbf{E}_{M R}$, when some required stencil is not present in $\Gamma^{n+}$. The details of such procedures are analyzed in the following sections.

\section{Error Analysis}

Let $\tilde{U}_{M R}^{n}$ be the result of the recursive prediction of $U_{M R}^{n}$ up to the finest level $J$. An error $\mathcal{E}^{n}$ is defined as the difference between the numerical solution $U^{n}$ given by the reference scheme based on $X_{J}$, and $\tilde{U}_{M R}^{n}$, i.e., $\mathcal{E}^{n}=U^{n}-\tilde{U}_{M R}^{n}$. Having in mind that $U^{n+1}=\mathbf{E} U^{n}$, the error $\mathcal{E}^{n+1}$ can be split into three terms

$$
\mathcal{E}^{n+1}=\left(\mathbf{E} U^{n}-\mathbf{E} \tilde{U}_{M R}^{n}\right)+\left(\mathbf{E} \tilde{U}_{M R}^{n}-{\widetilde{\mathbf{E}_{M R}}}_{M R}^{n+}\right)+\left({\widetilde{\mathbf{E}_{M R}}}_{M R}^{n+}-\tilde{U}_{M R}^{n+1}\right)
$$

The first term is the difference of the application of the reference scheme $\mathbf{E}$ on different data. Therefore, its behaviour depends on the stability properties of $\mathbf{E}$. The second term is the truncation error, which expresses the difference in first predicting and then applying the evolution operator, instead of applying the modified adaptive operator $\mathbf{E}_{M R}$ and then predicting. The last term corresponds to the thresholding error, since $U_{M R}^{n+1}$ is the result of the threshold application to $\mathbf{E} U_{M R}^{n+}$. Assume that the initial error $\mathcal{E}^{0}$, the thresholding and the truncation errors can be bounded by a control parameter $\epsilon$. If the evolution operator is a contraction, then it follows that

$$
\left\|\mathcal{E}^{n+1}\right\| \leq C(n+1) \epsilon \text {. }
$$

As explained previously, the control of the thresholding error relies on the stability of the prediction operator. However, regarding the truncation error, this is a more delicate task. The contributions to the truncation error may come from two sides. Despite the fact that $\tilde{U}_{M R}^{n}$ has no wavelet components outside $\Gamma^{n+}$, the action of $\mathbf{E}$ may activate some of them. According to [17], a careful refinement strategy may be performed to keep such kind of perturbation under control. However, inside $\Gamma^{n}+$, another source of error may arise if $\mathbf{E}$ is not calculated exactly. For instance, this could be the case if $\mathbf{E}$ is replaced by its adaptive version $\mathbf{E}_{M R}$. In [30,44], this kind of consistency error is analyzed for the case of interpolating MR schemes combined with a finite difference scheme $\mathbf{E}$ provided by a collocation formulation on the approximation spaces $V_{J}$. It is shown that the adaptive finite difference operator $\mathbf{E}_{M R}$ and $\mathbf{E}$ can produce the same result at $\Gamma^{n+}$, provided such SPR grids have appropriate cone-like structures. Consequently, in addition to the refinement strategy, these analyses indicate that some 
care must be taken concerning the adaptive grid structure in order to keep the truncation error under a certain accuracy limit.

\section{Sparse Point Representation Method}

The SPR method, introduced by $[49,50]$, is an adaptive finite difference strategy that combines the simplicity and accuracy of traditional finite difference schemes with the ability of wavelet coefficients in the characterization of local regularity of functions. The idea is to represent the functions by the point values corresponding to their significant wavelet coefficients. Typically, few points are found in each time step, the grid being coarse in smooth regions, and refined close to irregularities. At each point, spatial derivatives are discretized by uniform finite differences, using step size proportional to the point local scale. Eventually, stencils not present in the grid are approximated from coarser scales by using an interpolating subdivision scheme.

Other adaptive wavelet methods have been proposed with many similarities to the SPR method. For instance, the filter bank method by [82] and the second generation wavelet collocation method by [79] may be considered as generalizations of the SPR method. As it is well discussed in [82], one of the main attractive aspect of such classes of adaptive wavelet solvers is that they can be separated into two basic parts: the representation part and the operator part. The operator part is performed by finite differences on uniform grids which may be chosen by considering stability and consistency criteria. Therefore, they can be beneficiary of the considerable advances achieved in this area. The representation part is formulated in the context of wavelet data compression by means of a simple thresholding operation. This is a more recent topic, but a rigorous study of the effectiveness of such kind of non-linear approximation has already being established. We refer to [14] for a substantial survey on this subject.

This kind of separation of the solver into independent parts makes this approach general and flexible: it is simple to change the differential equation, the order of the finite difference method, the boundary conditions, the wavelet transform, etc. Consequently, it fits well into the object oriented programming philosophy.

However, as it usual in MR methods, one major weakness of the SPR method is the overhead involved in accessing interpolating neighboring stencils to compute finite differences on scattered grid points. One possible remedy is to decrease the overhead by losing some grid sparsity by the enforcement of some kind of grid structure. For instance, this is the principal idea of using graded tree structure, as described in the following sections. Another suggestion, by M. Holmstrom [49], is to use adaptive block representations (ABR). The computational domain is formed by non-overlapping blocks. Each block is a uniform grid, but the step size may change from one block to another. In the automatic construction of such block-grids, the wavelet coefficients are also used as the main tool to decide whether a block needs to be refined or may be coarsened. In order to avoid undesirable search communications between blocks during the computation of derivatives, layers of auxiliary neighbouring points are added around each block. In [49], the ABR method is applied to solve the 2D Navier-Stokes equations, for a flow over a flat plate, showing a boundary layer of increasing width, obtaining both CPU and memory compressions

\subsection{Adaptive Block Representation}

In this section we describe a general framework for the application of the ABR method in 2D, as established in [31]. The algorithms and data structure are formulated by using abstract concepts borrowed from quaternary trees (quad-trees). With this procedure, we expect to improve the understanding of the method and help the process of its computational implementation. The ability of the method is demonstrated by solving some test problems showing typical features of spikes, propagating fronts and the formation of sharp transition layers.

Let $X_{0}$ be the uniform grid on the rectangle $[0, L] \times[0, D]$ with spatial steps $h_{x}=\frac{L}{N_{x}}$ in the $x$-direction and $h_{y}=\frac{D}{N_{y}}$ in the $y$-direction. That is,

$$
X_{0}=\left\{\gamma=\left(k h_{x}, \ell h_{y}\right), 0 \leq k<N_{x}, 0 \leq \ell<N_{y}\right\} .
$$


Starting from $X_{0}$, a hierarchy of uniform grids $X_{j}$ are constructed by successive dyadic refinements

$$
X_{j}=\left\{\gamma=\left(k h_{x}^{j}, \ell h_{y}^{j}\right), 0 \leq k<N_{x}^{j}, 0 \leq \ell<N_{y}^{j}\right\},
$$

where $N_{x}^{j}=2^{j} N_{x}, N_{y}^{j}=2^{j} N_{y}, h_{x}^{j}=2^{-j} h_{x}$ and $h_{y}^{j}=2^{-j} h_{y}$. Thus, each $X_{j}$ is obtained from $X_{j-1}$ by midpoint insertions. This grid sequence can be organized in a quaternary tree structure (quad-tree).

\section{Quad-Tree Structure}

Let $\mathcal{B}_{j, \mu}$ be a generic block of $N_{x} \times N_{y}$ points in $X^{j}$ of type

$$
\mathcal{B}_{j, \mu}=\left\{\gamma=\mu+\left(k h_{j_{x}}, \ell h_{j_{y}}\right), 0 \leq k<N_{x}, 0 \leq \ell<N_{y}\right\}
$$

which is uniquely represented by its origin position $\mu$ and its scale level $j$. From $\mathcal{B}_{j, \mu}$, new blocks at level $j+1$ are obtained, firstly by dyadic refinement, and then by splitting into four parts

$$
\mathcal{B}_{j, \mu} \rightarrow \mathcal{S}\left(\mathcal{B}_{j, \mu}\right)=\left\{\mathcal{B}_{j+1, \mu_{0}}, \mathcal{B}_{j+1, \mu_{1}}, \mathcal{B}_{j+1, \mu_{2}}, \mathcal{B}_{j+1, \mu_{3}}\right\}
$$

If $\mu$ is the origin of the starting block, then the origins of the new blocks are $\mu_{00}=\mu, \mu_{01}=\mu+\left(0, \frac{N_{y}}{2} h_{j_{y}}\right)$, $\mu_{10}=\mu+\left(\frac{N_{x}}{2} h_{j_{x}}, 0\right)$, and $\mu_{11}=\mu+\left(\frac{N_{x}}{2} h_{j_{x}}, \frac{N_{y}}{2} h_{j_{y}}\right)$. Using the quad-tree terminology, the elements of the set $\mathcal{S}\left(\mathcal{B}_{j, \mu}\right)$ are called the children of the node $\mathcal{B}_{j_{\mu}}, \mathcal{B}_{j, \mu}$ is the mother of $\mathcal{B}_{j+1, \mu_{\kappa}}, \mathcal{B}_{j+1, \mu_{\kappa^{\prime}}}$ is son of $\mathcal{B}_{j, \mu}$, and $\mathcal{B}_{j+1, \mu_{\kappa}}$ is brother of $\mathcal{B}_{j+1, \mu_{\kappa^{\prime}}}$. One generation of a quad-tree block structure is illustrated in Figure 28.

a) Tree structure

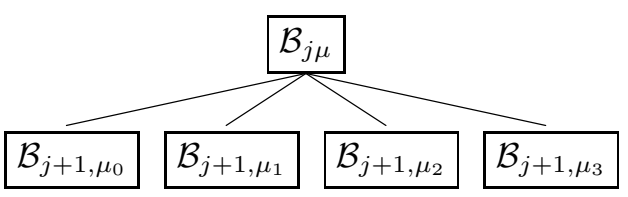

b)Grid structure

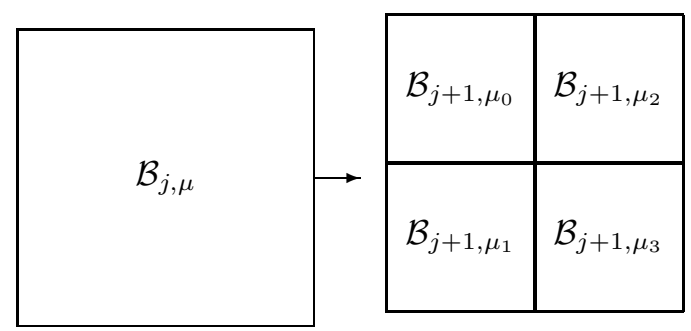

FiguRE 28. The representation of a block generation.

By defining $\mathcal{B}_{0, \mu_{0}}=X_{0}$ as the root of the tree, and performing $J$ generations, a complete quad-tree having $J+1$ levels is obtained. At each level $0 \leq j \leq J$ there exist $2^{2 j}$ blocks (nodes). Let us denote by $\mathcal{I}^{j}$ the set of block origin points at level $j$.

Incomplete quad-trees occur when at intermediary levels some nodes have no children. This leads to the concept of tree leaves: leaves are nodes that do not have children. 
In a complete block quad-tree, the leaves correspond to the blocks $\mathcal{B}_{J, \mu}$ at the last level. The union of these blocks constitutes the uniform grid at the finest scale

$$
X_{J}=\bigcup_{\mu \in \mathcal{I}^{J}} \mathcal{B}_{J, \mu} .
$$

In an incomplete block quad-tree, the leaves at intermediary levels $0<j<J$ correspond to blocks where the refinement process has been interrupted. Let $\Lambda_{j} \subset \mathcal{I}_{j}$ be the set of origin points associated to the leaf-blocks at level $j$. The union of such blocks forms a block-structured grid

$$
\mathcal{M}=\bigcup_{j=1}^{J} \bigcup_{\mu \in \Lambda_{j}} \mathcal{B}_{j, \mu}
$$

The diagram in Figure 29 illustrates a 4-level incomplete tree and its corresponding grid.

a)

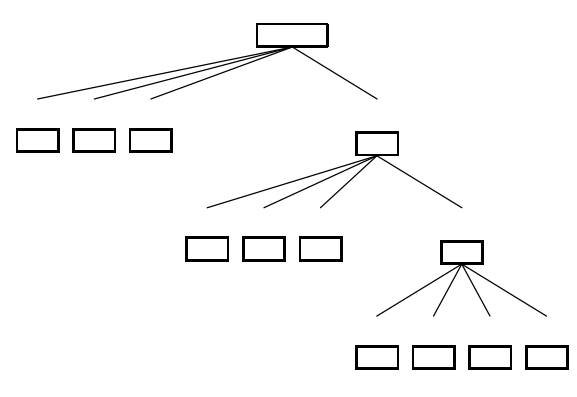

b)

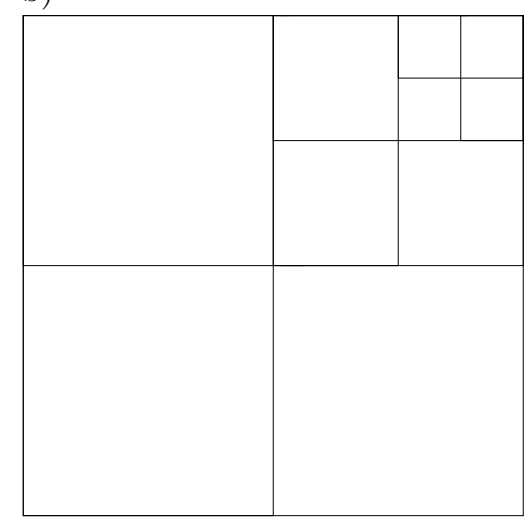

FiguRE 29. Incomplete 4-level quad-tree and its corresponding block-structured grid.

\section{Adaptive Construction of Block-Grids}

For a given block-structured grid $\mathcal{M}$, we shall denote by $f_{\mathcal{M}}$ the collection of point values of a given function $f(x, y)$ represented at $\mathcal{M}$. According to the tree structure of $\mathcal{M}, f_{\mathcal{M}}$ can be organized as a vector whose components are $N_{x} \times N_{y}$ matrices containing the data corresponding to the point values at the leaf-blocks $\mathcal{B}_{j, \mu}$.

The purpose of the ABR technique is to obtain representations $\left\{\mathcal{M}, f_{\mathcal{M}}\right\}$ as sparse as possible. This means that a small total number of blocks is found in an incomplete quad-tree $\mathcal{M}$, the leaves in lower levels (big scales) corresponding to smooth regions and those at higher levels (small scales) corresponding to irregularity regions. In the construction of a such an adaptive representation, the main tool is a local regularity indicator $i(\mathcal{B})$ to decide, at each generation, whether a given block should be a leaf, $i(\mathcal{B})=0$, or not, $i(\mathcal{B})=1$. Next, we shall describe how wavelet coefficients in an interpolatory multiresolution analysis can be used in the definition of $i(\mathcal{B})$. In the wavelet literature, there are several examples of multiresolution analyses that may be used as well. In fact, some of them offer convenient aspects, like shorter filters for the same order of polynomial cancellation (zero moments for the dual wavelets), as described in [82], or additional zero moments for the primal wavelets, like in the modified lifting scheme adopted by [79]. 


\section{Wavelet Indicators}

Given a generic block $\mathcal{B}_{j, \mu}$ at level $j$, define the set $\tilde{\mathcal{B}}_{j, \mu}$ as the completion of $\mathcal{B}_{j, \mu}$ by the inclusion of extra points in the right and upper lateral lines. That is,

$$
\tilde{\mathcal{B}}_{j, \mu}=\left\{\gamma=\mu+\left(k h_{j_{x}}, \ell h_{j_{y}}\right), 0 \leq k \leq N_{x}, 0 \leq \ell \leq N_{y}\right\} .
$$

To illustrate the process of filling up the right and up boundaries of the block grids, consider the block-grid shown in Figure 30 (a), which is formed by $4 \times 4$ blocks in three scale levels, and take the inner block $\mathcal{B}_{j+2, \beta}$. On its right side, the neighbor block $\mathcal{B}_{j+2, \delta}$ is placed at the same level. The right column of point values in $\tilde{\mathcal{B}}_{j+2, \beta}$ is just filled up by copying the left column of point-values of $\mathcal{B}_{j+2, \delta}$. The scale of the upper neighbor $\mathcal{B}_{j, \mu}$ is four times the scale of $\tilde{\mathcal{B}}_{j+2, \delta}$. Therefore, the upper row of $\tilde{\mathcal{B}}_{j+2, \beta}$ can only be partially filled up by taking point values that may be present in the lower row of $\mathcal{B}_{j, \mu}$. The remaining point-values are set by dyadic refinement, using only those point copied values.

It is clear that some restrictions need to be made relating the number of grid points in each block, the degree of interpolation and the maximum difference of scale levels in neighbouring blocks. For instance, in the particular case of Figure 30, with $4 \times 4$ points in each block, no more than two levels of difference is allowed. Furthermore, by allowing two scale levels of difference, as in the example shown in Figure 30, only linear prediction is possible.

(a)

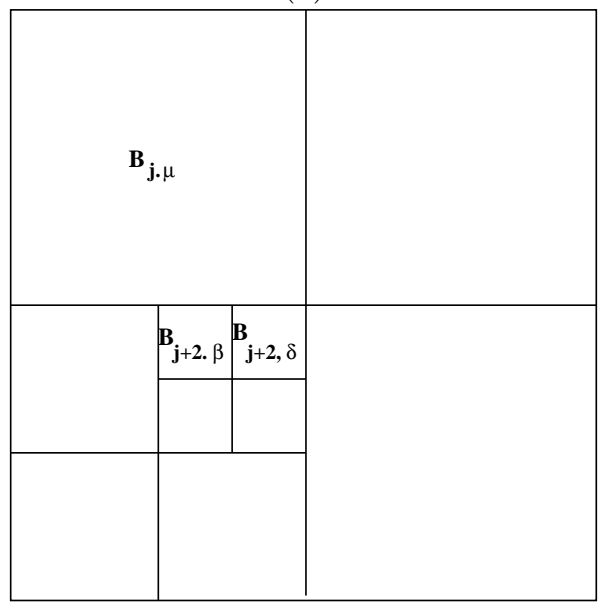

(b)
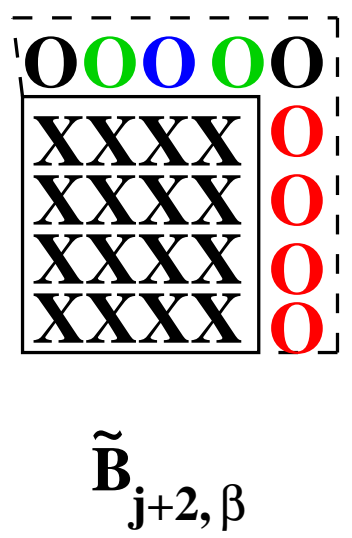

Figure 30. Example of how to fill up the right and upper block laterals. (a) Block position on the grid. Blocks have $4 \times 4$ points inside. (b) Complete block $\mathcal{B}_{j+2, \beta}$. Points in $\mathcal{B}_{j+2, \beta}$ are denoted by the symbol $\times$. Points in upper row which are filled up with data from block $\tilde{\mathcal{B}}_{j, \mu}$ are denoted by $O$, and those in the right column taken from $\mathcal{B}_{j+2, \delta}$ are denoted by 0 . In the upper row, the symbols $O$ and $O$ indicate the point values included by a two level dyadic refinement starting from ) points as stencils.

Consider the rectangular grid $\mathcal{R}_{j}=\tilde{\mathcal{B}}_{j, \mu}$ and its dyadic refinement $\mathcal{R}^{j+1}$ given by

$$
\mathcal{R}^{j+1}=\left\{\gamma=\mu+\left(k h_{x}^{j+1}, \ell h_{y}^{j+1}\right), 0 \leq k \leq 2 N_{x}, 0 \leq \ell \leq 2 N_{y}\right\}
$$

Note that $\mathcal{R}_{j+1}$ can also be expressed as

$$
\mathcal{R}_{j+1}=\bigcup_{\kappa=0}^{3} \tilde{\mathcal{B}}_{j+1, \mu_{\kappa}}
$$


where $\tilde{\mathcal{B}}_{j+1, \mu_{\kappa}}$ are the completion of the children of $\mathcal{B}_{j, \mu}$.

Let $f_{j+1}$ be the matrix containing the values of a given function $f(x, y)$ at the grid points in $\mathcal{R}_{j+1}$

$$
f_{j+1,(k, \ell)}=f\left(\mu+\left(k h_{x}^{j+1}, \ell h_{y}^{j+1}\right)\right) .
$$

On this grid block, consider the two-level MR transformation $f_{j+1} \leftrightarrow\left\{f_{j}, d_{j}^{(1)}, d_{j}^{(2)}, d_{j}^{(3)}\right\}$, as indicated in Section 2. As interpolation errors, wavelet coefficients are good indicators of local smoothness. This fact leads to the definition of a set index $i_{\epsilon}(\mathcal{B})$ which is based on the significance of the wavelet coefficients associated to the block $\mathcal{B}$, as compared to a certain given threshold $\epsilon$. By definition, $i_{\epsilon}\left(\mathcal{B}_{0,0}\right)=1$. Then, at level $j>0$, for each block $\mathcal{B}_{\mu}^{j}$ having set index equal to one, the children set indexes $i_{\epsilon}\left(\mathcal{B}_{j+1, \mu_{p, q}}\right)$ are computed according to the following strategy. First we perform the one-level wavelet transform in each rectangular grid $\mathcal{R}_{j+1}$, as described before, and consider $\mathbb{D}\left(\mathcal{B}_{j+1, \mu_{\kappa}}\right)$ as the set of those wavelet coefficients $d_{j,(k, \ell)}^{(i)}$ which are associated to the new points in $\tilde{\mathcal{B}}_{j+1, \mu_{\kappa}} \backslash \mathcal{R}_{j}$. If all these wavelet coefficients are not significant, this means that the function is smooth in this region. Consequently, the block does not need to be refined, and it will be a leaf-tree. On the other hand, if there exists at least one significant wavelet coefficient in the block, this means that the function is not represented there with the prescribed accuracy, and refinement is needed. Therefore, according to these principles, the set index $i_{\epsilon}$ is defined by

$$
i_{\epsilon}\left(\mathcal{B}_{j+1, \mu_{k}}\right)= \begin{cases}0 & \text { if }\left|d_{j,(k, \ell)}\right|<\epsilon \forall d_{j,(k, \ell)}^{(i)} \in \mathbb{D}\left(\mathcal{B}_{\mu_{p, q}^{j}}^{j+1}\right) \\ 1 & \text { otherwise. }\end{cases}
$$

Following this strategy, the adaptive grid construction ends when, at a certain level, all the analyzed blocks are leaves.

\subsubsection{Numerical Examples}

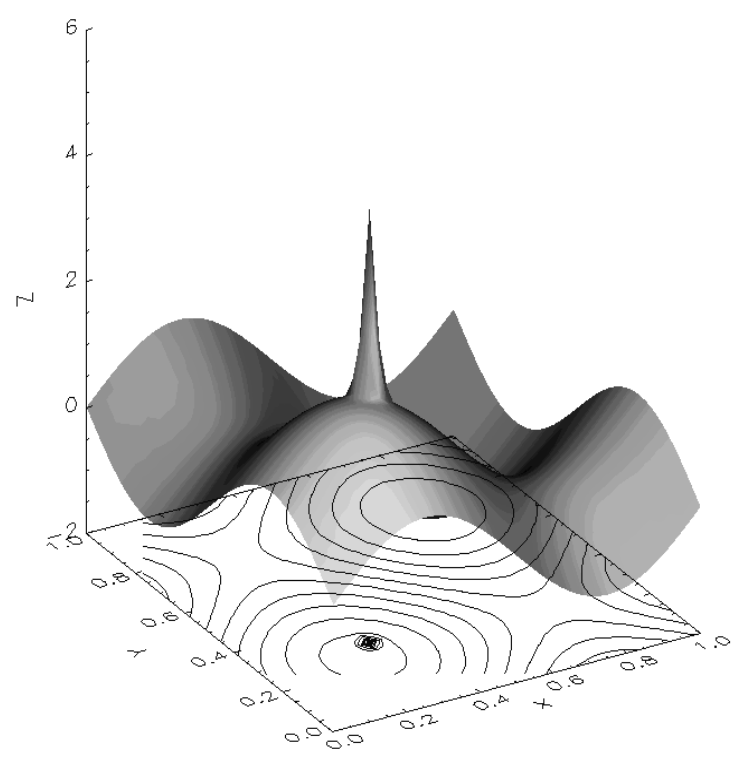

FiguRE 31. A smooth function with a superimposed spike function. 
The first example is for the sinusoidal with a superimposed spike function

$$
f(x, y)=3 \exp ^{-2500.0\left((x-0.3)^{2}+(y-0.3)^{2}\right)}+\sin (2 \pi x)+\sin (2 \pi y)
$$

with a singularity point at $(x, y)=(0.3,0.3)$, as illustrated in Figure 31 . For this function, the ABR grids $\mathcal{M}_{\epsilon}$ are represented in Figure 32. The wavelet coefficients are for cubic polynomial interpolation $(M=4)$, the blocks have $N_{x}=N_{y}=32$ and $\epsilon=10^{-3}, 10^{-4}$ and $10^{-5}$. As $\epsilon$ becomes smaller, more refined blocks are tended to concentrate near the singularity region. As indicated in Table 1, the number of blocks grows as $\epsilon$ becomes smaller. However, if the number of points in the ABR grid $\mathcal{M}_{\epsilon}$ is compared to number of points in the uniformed mesh $\mathcal{X}_{\ell}$, in which $\ell$ is the finest scale level present in $\mathcal{M}_{\ell}$, then the ratio $\sharp\left(\mathcal{M}_{\epsilon}\right) / \sharp\left(X_{\ell}\right)$ reduces very quickly as $\epsilon$ decreases.
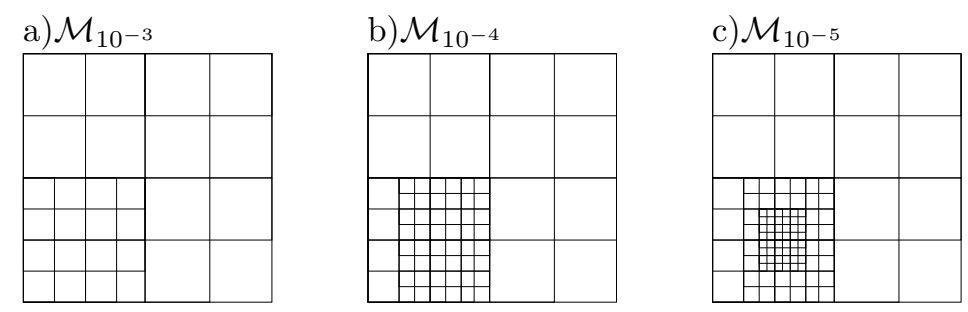

Figure 32. ABR for the spike function.

TABLE 1. Efficiency in ABR for the spike.

\begin{tabular}{c||c|r|r|r}
\hline \hline$\epsilon$ & $\sharp$ blocks & $\sharp\left(\mathcal{M}_{\epsilon}\right)$ & $\sharp\left(X_{\ell}\right)$ & $\sharp\left(\mathcal{M}_{\epsilon}\right) / \sharp\left(X_{\ell}\right)$ \\
\hline $10^{-5}$ & 100 & 102400 & 2097152 & 0.05 \\
$10^{-4}$ & 64 & 65536 & 262144 & 0.25 \\
$10^{-3}$ & 28 & 28672 & 65536 & 0.43 \\
\hline \hline
\end{tabular}

As a second example, we shall consider the oblique-front function

$$
f(x, y)=1-\tanh (25 x+5(y-1))
$$

that exhibits abrupt changes close to the line $25 x+5(y-1)=0$, as displayed in Figure 33 . Therefore, the most refined blocks in the $\mathrm{ABR}$ grids $\mathcal{M}_{\epsilon}$ are expected to be placed around the oblique-front region, as $\epsilon$ decreases. This behaviour is illustrated in Figure 34 for the same $\epsilon$ parameters as in the previous example. An indication of the efficiency of the ABR method for the oblique-front function can be obtained from the data in Table 2 . Their comparison with the corresponding results in Table 1 implies that, given an accuracy $\epsilon$, the spike ABR meshes $\mathcal{M}_{\epsilon}$ need higher resolution levels and more blocks than in the oblique-front case. Despite of that, the efficiency ratio is better for the spike function.

Fixing $\epsilon=10^{-3}$ and the block size $32 \times 32$, ABR grids $\mathcal{M}_{10^{-3}}$ for the oblique front are presented in Figure 35 (a) and (b), for different interpolation order. The number of blocks occurring with linear interpolation $(M=2)$ is more than twice than with cubic interpolation $(M=4)$. Fixing $M=4$ and reducing the block size to $N_{x}=N_{y}=16$, Figure 35 (c) indicates that the number of blocks in $\mathcal{M}_{10^{-3}}$ increases. However, despite of the smaller block size, the total number of points also increases. A similar behaviour also occurs for different values of the truncation parameter $\epsilon$. 


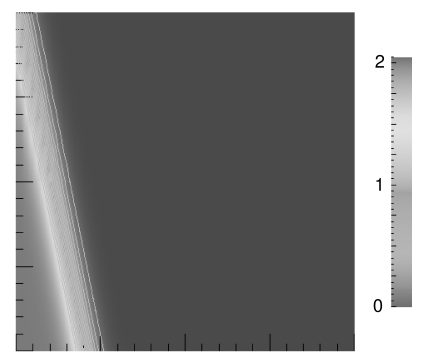

Figure 33. Oblique-front function.

a) $\mathcal{M}_{10^{-3}}$

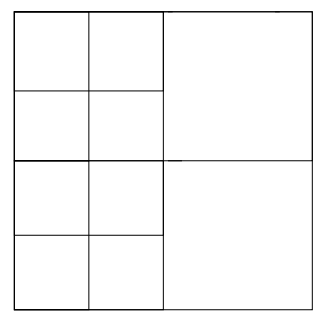

b) $\mathcal{M}_{10^{-4}}$

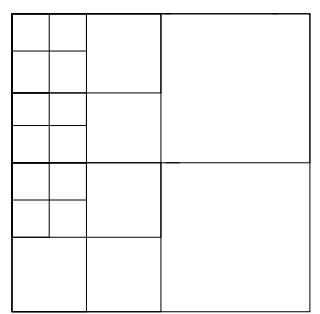

c) $\mathcal{M}_{10^{-5}}$

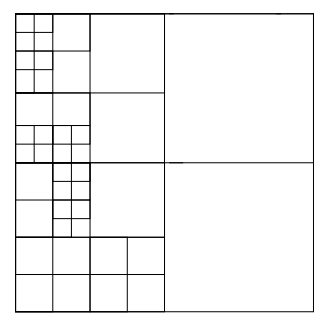

FiguRE 34. ABR for the oblique-front function.

TABLE 2. Efficiency in ABR for the oblique-front.

\begin{tabular}{c||c|r|r|r}
\hline \hline$\epsilon$ & $\sharp$ blocks & $\sharp\left(\mathcal{M}_{\epsilon}\right)$ & $\sharp\left(\mathcal{X}_{\ell}\right)$ & $\sharp\left(\mathcal{M}_{\epsilon}\right) / \sharp\left(X_{\ell}\right)$ \\
\hline $10^{-5}$ & 44 & 45056 & 262144 & 0.17 \\
$10^{-4}$ & 19 & 19456 & 65536 & 0.30 \\
$10^{-3}$ & 10 & 10240 & 16384 & 0.60 \\
$10^{-2}$ & 4 & 4096 & 4096 & 1.00 \\
\hline \hline
\end{tabular}

\subsection{Operations on Block-Structured Grids}

For the application of ABR method in the numerical solution of partial differential equations, there are some typical operations involved: grid refinement and coarsening, functional operations and differentiation.

\section{Refining and Coarsening}

The automatic adaptation of the grid structure during time evolution requires a simple procedure for refining or coarsening the grids. For block-structured grids, these operations are easily defined in the context of quadtrees by means of tree extension or reduction. In a general sense, if $\mathcal{A}$ and $\tilde{\mathcal{A}}$ are two quad-trees such as $\mathcal{A} \subset \tilde{\mathcal{A}}$, then $\tilde{\mathcal{A}}$ is an extension of $\mathcal{A}$, or $\mathcal{A}$ is a reduction of $\tilde{\mathcal{A}}$. Specifically, the extension and reduction operations of interest are described next.

- Tree Extension (Grid Refinement) : $\mathcal{A} \stackrel{\mathcal{E}}{\rightarrow} \tilde{\mathcal{A}}$ 
a)

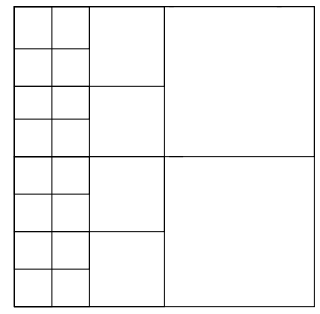

b)

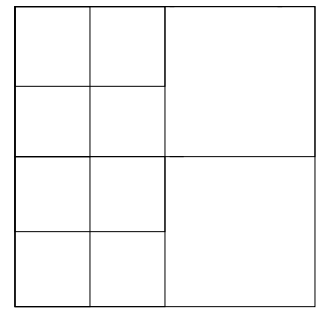

c)

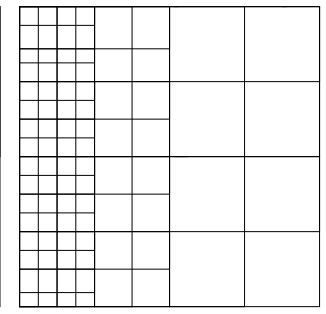

FIGURE 35. Oblique front representation in block adaptive mesh $\mathcal{M}_{10^{-3}}$ : a) $M=2$, with 22 blocks $32 \times 32$; b) $M=4$, with 10 blocks $32 \times 32$ and c) $M=4$, with 88 blocks $16 \times 16$.

An extended tree $\tilde{\mathcal{A}}$ may be obtained by adding a new block generation to all leaves of the tree $\mathcal{A}$. Figure 36 shows an example of a block structured grid $\mathcal{M}$ and its corresponding extension $\tilde{\mathcal{M}}$. Given the representation $\left(\mathcal{M}, f^{\mathcal{M}}\right)$, the functional values $f^{\tilde{\mathcal{M}}}$ at the extended grid $\tilde{\mathcal{M}}$ may be not available. In such case, each missing value in $f^{\tilde{\mathcal{M}}}$ is obtained by the interpolatory refinement scheme.
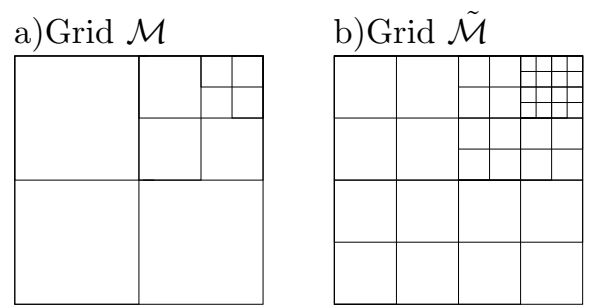

FigurE 36. Extension operation (a) Original grid; (b) Extended grid.

- Thresholding (Tree Reduction; Grid Coarsening): $\tilde{\mathcal{A}} \stackrel{\mathcal{I}_{\epsilon}}{\longrightarrow} \mathcal{A}$

As done for the grid construction, the reduction operator $\mathcal{T}_{\epsilon}$ is characterized by a regularity indicator $\imath_{\epsilon}(\mathcal{B})$. All leaves in $\tilde{\mathcal{A}}$, whose three brothers are also leaves, are tested. If one of these leaves and their brothers have $i_{\epsilon}=0$, these nodes are removed from the tree structure. Consequently, their mother becomes a new leaf-node. When this particular case happens, the leaf-structure changes. Therefore, the reduction process must be executed again.

\section{Functional Operations}

Operations between two functions represented in block-structured grids are straightforward point-wise evaluations if their grids coincide. Otherwise, it is necessary to extend both grids in order to get representations in a common grid.

\section{Differentiation}

The idea is to use finite difference operators with uniform spacing in each block of the grid $\mathcal{M}$. For blocks at level $\jmath$, partial derivatives in the $x$-direction are discretized with spacing $h_{x}^{\jmath}$, and in the $y$-direction with $h_{y}^{\jmath}$. For points close to the block $\tilde{\mathcal{B}}_{j, \mu}$ laterals, stencil information from neighbouring blocks are required.

To increase the independence of the blocks, the process of block construction should consider addition extra rows and columns around the block $\tilde{\mathcal{B}}_{j, \mu}$ laterals. These extra points are filled up using data from neighboring 
blocks. However, depending on the difference of scale levels, the needed informations may belong to the neighbor of a neighbor, or even further. On the implemented SPR code, to simplify the computational search procedure for block neighborhood, the allowed difference of levels must be restricted in order to guaranties that the necessary extra data for the differentiation can be taken from closest block-neighborhood. For instance, if the block size is $32 \times 32$ and the differentiation needs two extra rows/columns, then no more than 4 levels of difference are allowed on neighbor blocks. These extra points are the so called ghost points,and they are used just to the differentiation, i.e., no time evolution is performed on them. The importance in maintaining this block independence is crucial for the implementation of the method on parallel computers.

\subsection{Numerical Examples}

A solver that performs the ABR method is implemented using a $C^{++}$object oriented paradigm programming. All the simulations are for 4 -th order interpolation, finite differences and Runge-Kutta ODE solver. The time step is dynamically chosen according to the smallest scale $h_{\text {min }}^{n}$ present in the extended adaptive mesh $\mathcal{M}^{n+}$, such that $h_{\min }^{n} / \Delta t^{n}=\lambda$.

\subsubsection{Oblique Front}

Let us consider the advection-diffusion equation

$$
\frac{\partial u}{\partial t}+\frac{\partial u}{\partial x}+\frac{\partial u}{\partial y}-\frac{\partial^{2} u}{\partial x^{2}}-\frac{\partial^{2} u}{\partial y^{2}}=\mathbf{F}
$$

for $t \geq 0,(x, y) \in[0,1] \times[0,1]$. Given the forcing term

$$
\mathbf{F}(x, y, t)-\beta \operatorname{sech}^{2}(z)-2\left(\alpha^{2}+\beta^{2}\right) \operatorname{sech}^{2}(z) \tanh (z),
$$

where $z=25(x-t)+5(y-1)$, the exact solution is

$$
\mathcal{U}=1-\tanh (z) .
$$

It describes a propagating steep front moving to the right. Using the boundary values of this exact solution, Figure 37 exhibits some typical features for the resulting numerical solution and the corresponding computational adaptive block-grids. The parameters for this simulation are alpha $=25, \beta=5, \epsilon=10^{-2}, N_{x}=N_{y}=16$ and $\lambda=10^{-3}$. Figure 38 shows how the number of blocks in the adaptive mesh evolves during the simulation. The maximum number of blocks occurs when the front crosses the center of the region.

\subsubsection{Sharp Transition Layers}

The following results are for the Burger's equation

$$
\frac{\partial u}{\partial t}+u \frac{\partial u}{\partial x}+u \frac{\partial u}{\partial y}-\mu \frac{\partial^{2} u}{\partial x^{2}}-\mu \frac{\partial^{2} u}{\partial y^{2}}=0,
$$

$t \geq 0,(x, y) \in[0,1] \times[0,1]$ and $\mu=10^{-2}$, with periodic boundary conditions and initial data

$$
u(x, y, 0)=\sin (2 \pi x) \sin (2 \pi y) .
$$

Since negative and positive features move in opposite directions, this example shows sharp transition layers, as time evolves. In the present simulation, the parameters are $\epsilon=10^{-5}, N_{x}=N_{y}=32$ and $\lambda=5 \times 10^{-2}$. Figure 39 exhibits some typical features of the numerical solution and the corresponding extended adaptive grids. Initially, the function is smooth and the mesh is totally uniform with 16 blocks. At $t \approx 0.9$, the application of the ABR method produces an irregular mesh with refined blocks close to the sharp transition regions. Figure 40 shows how the number of blocks in the adaptive meshes increases according to the steepness of the solution during the simulation. 
a) $t=0.35$
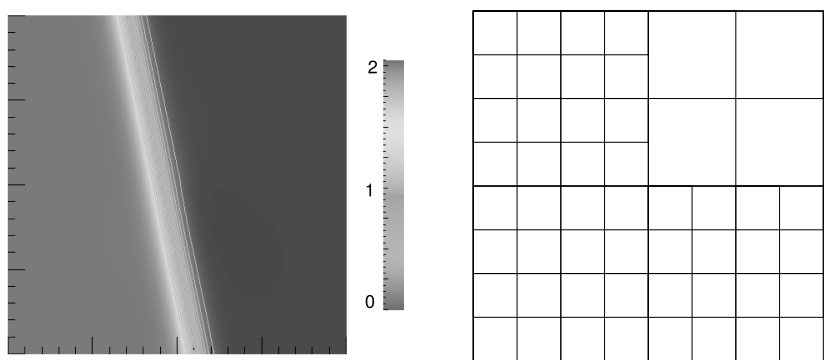

b) $t=0.45$
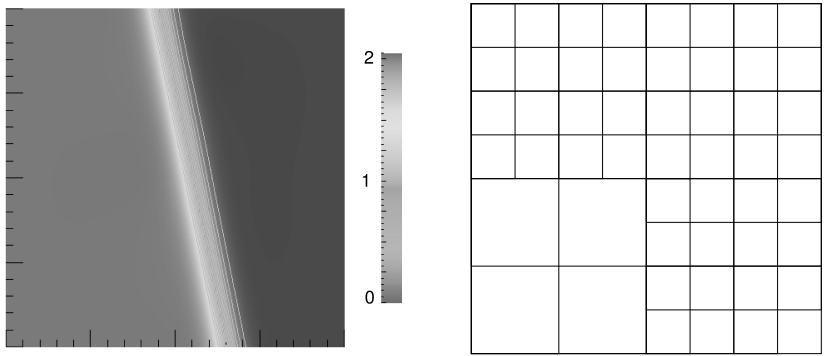

c) $t=0.70$
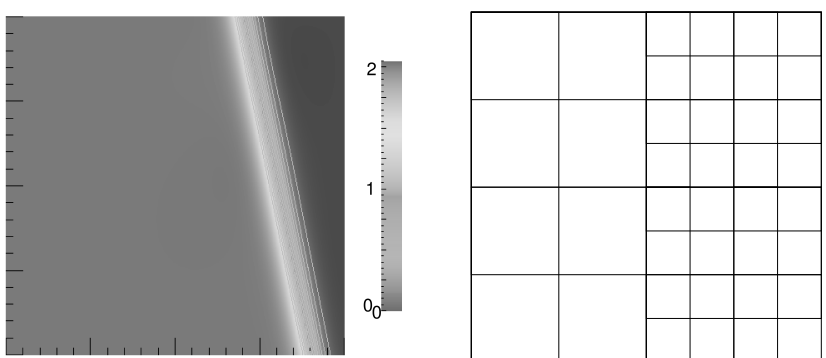

Figure 37. Oblique front isosurfaces (left), at three time instants from top to bottom, with corresponding sparse block-grid (right).

\section{Finite Volume Multiresolution Schemes}

\subsection{Finite Volume Discretization on Uniform Grids}

We consider conservation laws for $(x, t) \in \Omega \times[0,+\infty), \Omega \subset \Re^{d}$, of the form

$$
\frac{\partial u}{\partial t}=\mathcal{D}(u, \nabla u)
$$




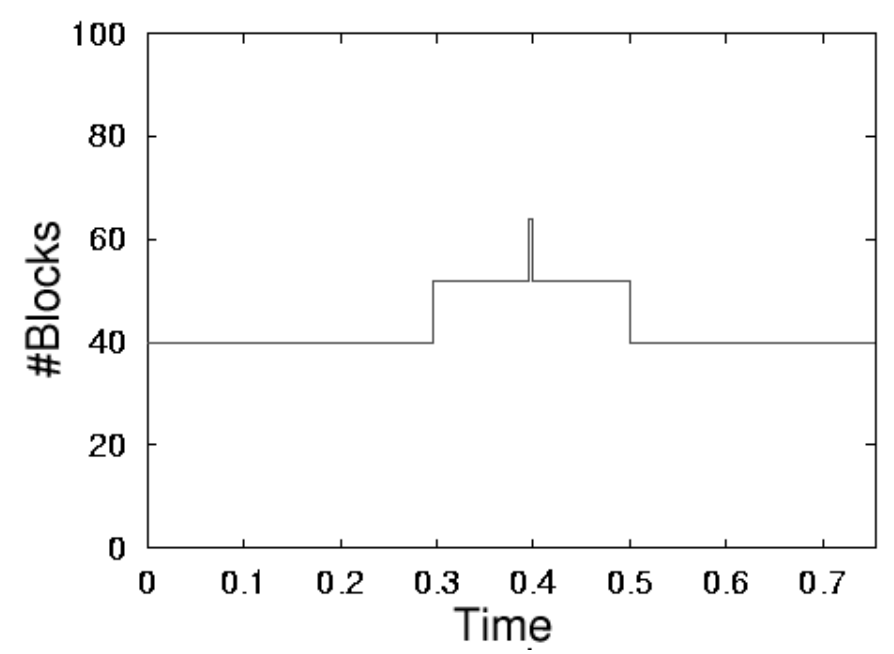

Figure 38. Evolution of the number of blocks in the adaptive meshes for the oblique front.

(a) $t=0$
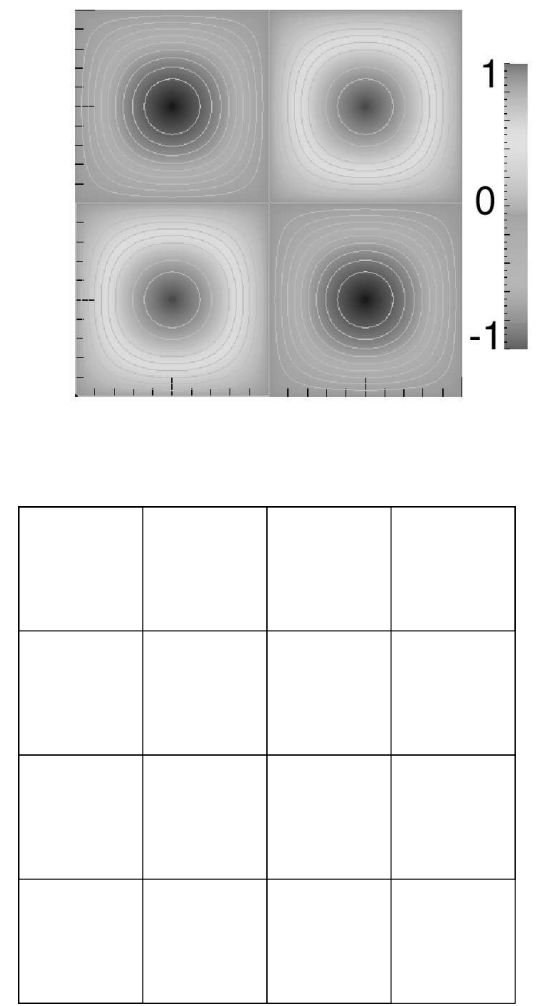

(b) $t=0.09$
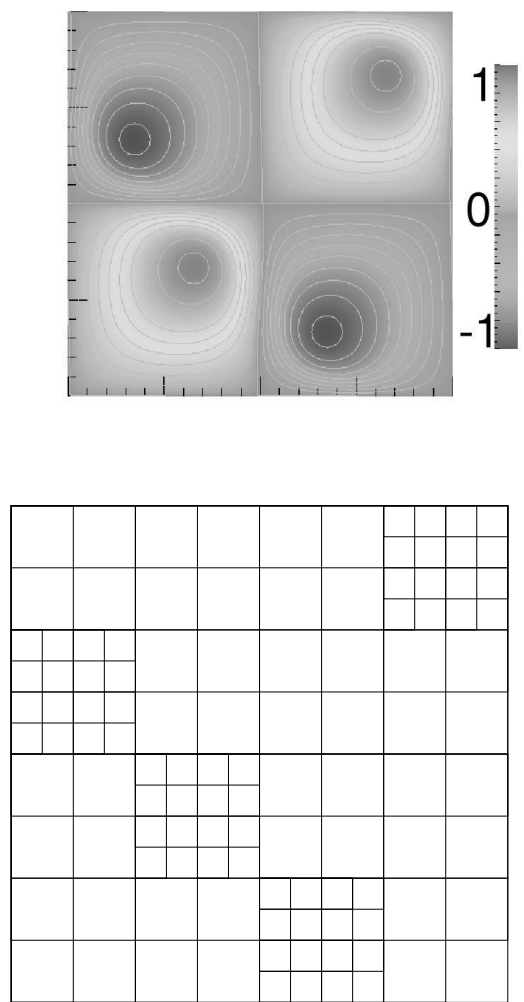

Figure 39. Sharp transition layer formation. Solution and adaptive block mesh at different times. 


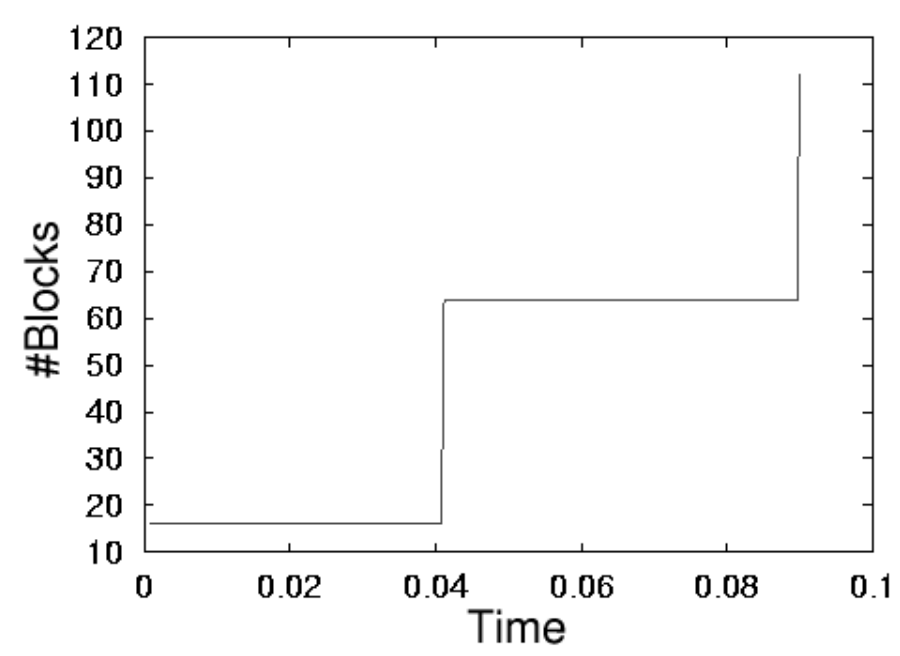

FiguRE 40. Evolution of the number of blocks for the sharp transition layer.

with initial condition $u(x, 0)=u_{0}(x)$, and appropriate boundary conditions. The operators are of type $\mathcal{D}(u, \nabla u)=-\nabla \cdot F(u, \nabla u)+S(u)$, formed by divergence and source terms. For the applications of these lecture notes, the flux function contains advective and diffusive parts of the form $F(u, \nabla u)=f(u)-\nu \nabla u$, with constant diffusion coefficient $\nu \geq 0$. For instance, we consider typical models for fluid dynamics and combustion problems:

- 1D convection-diffusion equation: $f(u)=c u$, and $S(u)=0$, where $(c>0)$;

- $1 \mathrm{D}$ viscous Burgers equation: $f(u)=\frac{u^{2}}{2}$ and $S(u)=0$;

- Reaction-diffusion equation: $f(u)=0$ and $S(u)=\frac{\beta^{2}}{2}(1-u) \exp \frac{\beta(1-u)}{\alpha(1-u)-1}$, where $(\alpha>0, \beta>0)$.

To set up the discretization model, we use a classical finite volume formulation in the standard conservative form. Let us consider a partition of the computational domain $\Omega$ into cells $\left(\Omega_{k}\right)_{k \in \Lambda}, \Lambda=\left\{1, \ldots, k_{\max }\right\}$. Let $\bar{u}_{k}(t)$ be the cell-average value of $u$ on $\Omega_{k}$ at instant $t$,

$$
\bar{u}_{k}(t)=\frac{1}{\left|\Omega_{k}\right|} \int_{\Omega_{k}} u(x, t) d x
$$

where $\left|\Omega_{k}\right|=\int_{\Omega_{k}} d x$ is the volume of the cell. Integrating the differential equation on $\Omega_{k}$ yields

$$
\int_{\Omega_{k}} \frac{\partial u}{\partial t}(x, t) d x=\int_{\Omega_{k}} \mathcal{D}(u(x, t), \nabla u(x, t)) d x
$$

i.e.,

Applying the divergence theorem, we get

$$
\frac{\partial \bar{u}_{k}}{\partial t}(t)=\overline{\mathcal{D}}_{k}(t)
$$

$$
\overline{\mathcal{D}}_{k}(t)=-\frac{1}{\left|\Omega_{k}\right|} \int_{\partial \Omega_{k}} F[u(x, t), \nabla u(x, t)] \cdot \sigma_{k}(x) d x+\bar{S}_{k}(t)
$$

where $\sigma_{k}(x)$ denotes the outer normal vector to $\Omega_{k}$. 
For time discretization, we use an explicit second-order accurate Runge-Kutta (RK2) scheme. Denoting by $\Delta t$ the time step and by $\bar{u}_{k}^{n}=\bar{u}_{k}\left(t^{n}\right)$, where $t^{n}=n \Delta t$, the RK2 scheme used here has the form

$$
\begin{aligned}
\bar{u}_{k}^{*} & =\bar{u}_{k}^{n}+\Delta t \overline{\mathcal{D}}_{k}^{n} \\
\bar{u}_{k}^{n+1} & =\frac{1}{2}\left[\bar{u}_{k}^{n}+\bar{u}_{k}^{*}+\Delta t \overline{\mathcal{D}}_{k}^{*}\right]
\end{aligned}
$$

Denoting by $\bar{U}^{n}$ the vector $\left(\bar{u}_{k}^{n}\right)_{k \in \Lambda}$, the discrete time evolution operator $\overline{\mathbf{E}}(\Delta t)$ is defined by

$$
\bar{U}^{n+1}=\overline{\mathbf{E}}(\Delta t) \cdot \bar{U}^{n}
$$

where

$$
\overline{\mathbf{E}}(\Delta t)=\mathbf{I}+\frac{\Delta t}{2}[\overline{\mathcal{D}}+\overline{\mathcal{D}}(\mathbf{I}+\Delta t \overline{\mathcal{D}})]
$$

and $\mathbf{I}$ denotes the identity operator.

A variety of methods can be distinguished in the literature, mainly, by the way the discretization of the operator $\overline{\mathcal{D}}$ is defined. Typically, advective and diffusive terms are approximated differently.

In the following, we consider a fixed time $t^{n}$, but the superscript $n$ is omitted everywhere. For the $1 \mathrm{D}$ case, $\Omega_{k}$ is a segment $\left[x_{k-\frac{1}{2}}, x_{k+\frac{1}{2}}\right]$ with step size $\Delta x_{k}=x_{k+\frac{1}{2}}-x_{k-\frac{1}{2}}$. Eq. (16) becomes

$$
\overline{\mathcal{D}}_{k}=-\frac{1}{\Delta x_{k}}\left(\bar{F}_{k+\frac{1}{2}}-\bar{F}_{k-\frac{1}{2}}\right)+\bar{S}_{k}
$$

For the advective part, we use Roe's scheme [64] with a second-order ENO interpolation, whereas, for the diffusive part, we choose a second-order accurate centred scheme. Bihari [9] showed that the resulting global scheme, which is non-linear, is second-order accurate in space.

$$
\bar{F}_{k+\frac{1}{2}}=f^{R}\left(\bar{u}_{k+\frac{1}{2}}^{-}, \bar{u}_{k+\frac{1}{2}}^{+}\right)-\nu \frac{\bar{u}_{k+1}-\bar{u}_{k}}{\Delta x_{k+\frac{1}{2}}}
$$

where $\Delta x_{k+\frac{1}{2}}=\frac{1}{2}\left(\Delta x_{k}+\Delta x_{k+1}\right)$

The term $f^{R}$ denotes, for the advective part, Roe's approximate solution to the Riemann problem given the left $(-)$ and right $(+)$ values of $u$. Its scalar version is given by

$$
f^{R}\left(u^{-}, u^{+}\right)=\frac{1}{2}\left(f\left(u^{-}\right)+f\left(u^{+}\right)-\left|a\left(u^{-}, u^{+}\right)\right|\left(u^{+}-u^{-}\right)\right)
$$

where

$$
a\left(u^{-}, u^{+}\right)=\left\{\begin{array}{lll}
\frac{f\left(u^{+}\right)-f\left(u^{-}\right)}{u^{+}-u^{-}} & \text {if } & u^{-} \neq u^{+} \\
f^{\prime}\left(u^{-}\right) & \text {if } & u^{-}=u^{+}
\end{array}\right.
$$

The left and right terms, $\bar{u}_{k+\frac{1}{2}}^{-}$and $\bar{u}_{k+\frac{1}{2}}^{+}$respectively, are computed using a second-order ENO interpolation

$$
\begin{aligned}
& \bar{u}_{k+\frac{1}{2}}^{-}=\bar{u}_{k}+\frac{1}{2} M\left(\bar{u}_{k+1}-\bar{u}_{k}, \bar{u}_{k}-\bar{u}_{k-1}\right) \\
& \bar{u}_{k+\frac{1}{2}}^{+}=\bar{u}_{k+1}+\frac{1}{2} M\left(\bar{u}_{k+2}-\bar{u}_{k+1}, \bar{u}_{k+1}-\bar{u}_{k}\right)
\end{aligned}
$$

where $M$ is the Min-Mod limiter, which chooses the minimal slope between the left and right sides, i.e.

$$
M(a, b)=\left\{\begin{array}{l}
a \text { if }|a| \leq|b| \\
b \text { if }|a|>|b|
\end{array}\right.
$$


The source term is approximated by $\bar{S}_{k} \approx S\left(\bar{u}_{k}\right)$. For a general non-linear source term, this choice yields a second-order accuracy.

Extension to higher dimension in Cartesian geometries is performed through a tensor product approach. For the $2 \mathrm{D}$ case, $\Omega_{k, m}$ is a rectangle with a volume of size $\left|\Omega_{k, m}\right|=\Delta x_{k} \Delta y_{m}$. Eq. (15) can be written as

$$
\frac{\partial \bar{u}_{k, m}}{\partial t}(t)=\overline{\mathcal{D}}_{k, m}(t)
$$

where

$$
\overline{\mathcal{D}}_{k, m}=-\frac{1}{\Delta x_{k}}\left(\bar{F}_{k+\frac{1}{2}, m}-\bar{F}_{k-\frac{1}{2}, m}\right)-\frac{1}{\Delta y_{m}}\left(\bar{F}_{k, m+\frac{1}{2}}-\bar{F}_{k, m-\frac{1}{2}}\right)+\bar{S}_{k, m} .
$$

The same numerical flux as in the $1 \mathrm{D}$ case is applied in each direction.

$$
\begin{aligned}
& \bar{F}_{k+\frac{1}{2}, j}=f^{R}\left(\bar{u}_{k+\frac{1}{2}, j}^{-}, \bar{u}_{k+\frac{1}{2}, j}^{+}\right)-\nu \frac{\bar{u}_{k+1, j}-\bar{u}_{k, m}}{\Delta x_{k+\frac{1}{2}}} \\
& \bar{F}_{k, m+\frac{1}{2}}=f^{R}\left(\bar{u}_{k, m+\frac{1}{2}}^{-}, \bar{u}_{k, m+\frac{1}{2}}^{+}\right)-\nu \frac{\bar{u}_{k, m+1}-\bar{u}_{k, m}}{\Delta y_{m+\frac{1}{2}}}
\end{aligned}
$$

where $\Delta x_{k+\frac{1}{2}}=\frac{1}{2}\left(\Delta x_{k}+\Delta x_{k+1}\right)$ and $\Delta y_{m+\frac{1}{2}}=\frac{1}{2}\left(\Delta y_{m}+\Delta y_{m+1}\right)$.

Analogously, for the $3 \mathrm{D}$ case, $\Omega_{k, m, \ell}$ is a rectangle parallelepiped with a volume of size $\left|\Omega_{k, m, \ell}\right|=\Delta x_{k} \Delta y_{m} \Delta z_{\ell}$. Hence we get

$$
\frac{\partial \bar{u}_{k, m, \ell}}{\partial t}(t)=\overline{\mathcal{D}}_{k, m, \ell}(t)
$$

where

$$
\begin{aligned}
\overline{\mathcal{D}}_{k, m, \ell}= & -\frac{1}{\Delta x_{k}}\left(\bar{F}_{k+\frac{1}{2}, m, \ell}-\bar{F}_{k-\frac{1}{2}, m, \ell}\right)-\frac{1}{\Delta y_{m}}\left(\bar{F}_{k, m+\frac{1}{2}, k}-\bar{F}_{k, m-\frac{1}{2}, k}\right) \\
& -\frac{1}{\Delta z_{\ell}}\left(\bar{F}_{k, m, \ell+\frac{1}{2}}-\bar{F}_{k, m, \ell-\frac{1}{2}}\right)+\bar{S}_{k, m, \ell} .
\end{aligned}
$$

The fluxes are in this case

$$
\begin{aligned}
& \bar{F}_{k+\frac{1}{2}, m, \ell}=f^{R}\left(\bar{u}_{k+\frac{1}{2}, m, \ell}^{-}, \bar{u}_{k+\frac{1}{2}, m, \ell}^{+}\right)-\nu \frac{\bar{u}_{k+1, m, \ell}-\bar{u}_{k, m, \ell}}{\Delta x_{k+\frac{1}{2}}} \\
& \bar{F}_{k, m+\frac{1}{2}, \ell}=f^{R}\left(\bar{u}_{k, m+\frac{1}{2}, \ell}^{-}, \bar{u}_{k, m+\frac{1}{2}, \ell}^{+}\right)-\nu \frac{\bar{u}_{k, m+1, \ell}-\bar{u}_{k, m, \ell}}{\Delta y_{m+\frac{1}{2}}} \\
& \bar{F}_{k, m, \ell+\frac{1}{2}}=f^{R}\left(\bar{u}_{k, m, \ell+\frac{1}{2}}^{-}, \bar{u}_{k, m, \ell+\frac{1}{2}}^{+}\right)-\nu \frac{\bar{u}_{k, m, \ell+1}-\bar{u}_{k, m, \ell}}{\Delta z_{\ell+\frac{1}{2}}}
\end{aligned}
$$

where $\Delta x_{k+\frac{1}{2}}=\frac{1}{2}\left(\Delta x_{k}+\Delta x_{k+1}\right), \Delta y_{m+\frac{1}{2}}=\frac{1}{2}\left(\Delta y_{m}+\Delta y_{m+1}\right)$, and $\Delta z_{\ell+\frac{1}{2}}=\frac{1}{2}\left(\Delta z_{\ell}+\Delta z_{\ell+1}\right)$.

We shall also consider simulations for the Euler equations of gas dynamics, for which upwind AUSM+ schemes [55] are adopted

\subsection{Conservative Fully Adaptive Multiresolution Scheme}

As described in Section 2, the principle of the multiresolution analysis for cell averages is to represent a set of data given on a fine grid as values on a coarser grid plus a series of differences at different levels of nested dyadic grids. The differences, the wavelet contributions, contain the information of the solution when going from a coarse to a finer grid. To apply this framework for the definition of adaptive FV schemes for partial differential equations, we must have discretizations in a hierarchy of embedded partitions of the computational domain into cells $\Omega_{j, k}$, at different scale levels $j$. The idea is to use the wavelet coefficients as local regularity 
indicators, and to represent the numerical solutions by the cell averages on adaptive partitions formed by cells corresponding to their significant wavelet coefficients. Typically, few information is required in each time step, the adaptive grids being coarse where the solution is smooth, and refined close to irregularities. But to have an efficient code, the data structure needs to be organized as a dynamic graded tree if one wants to compress data, while still being able to navigate through it.

\section{Dynamic Graded Tree}

In the wavelet terminology, a graded tree structure corresponds to the adaptive approximation. Its difference with the classical non-linear approximation is that the connectivity in the tree structure is always ensured. In other words, no hole is admitted inside the tree. DeVore [29] showed that the difference between both approximations is negligible in terms of required nodes.

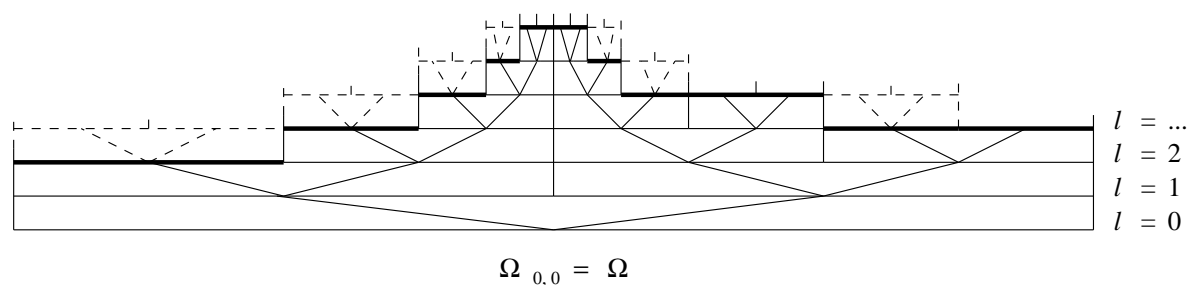

Figure 41. Example of graded tree data structure in $1 \mathrm{D}$ for $s=1, s^{\prime}=2$.

Following [17], we first introduce a terminology to define the tree structure.

- The root is the basis of the tree;

- A node is a element of the tree. Here, every cell, when existing, can be considered as a node;

- A parent node has $2^{d}$ children nodes, $d$ being the space dimension of the problem;

- The children nodes of the same parent are called brothers;

- A given node has nearest neighbours in each direction, called the nearest cousins. The brothers can also be considered as nearest cousins;

- Given a child node, the nearest cousins of the parent node are called the nearest uncles;

- A node is called a leaf when it has no children;

- In order to compute the ingoing and outgoing fluxes of a given leaf, we need its nearest cousins. When one of them is not existing, it is created as virtual leaf. A virtual leaf is not considered as an existing node and is only used for flux computations. As a consequence, no time evolution is made on it.

Figure 41 illustrates the graded tree structure in 1D. The standard nodes are represented by a thin line, the leaves by a bold line, the virtual leaves by a dotted line.

A dynamic tree is a tree which changes in time. When needed, some nodes can be added or removed. To remain graded, it must respect the following conditions :

- When a child is created, all its brothers are also created in the same time;

- A given node has always its $s$ nearest uncles in each direction, diagonal included. When not existing, create them as nodes ;

- A given node has always its $s^{\prime}$ nearest cousins in each direction. When not existing, create them as virtual leaves ;

As a consequence, a node can be removed only if all its brothers can also be removed, and if it is not the nearest uncle of an existing node. The number of nearest cousins $s^{\prime}$ depends on the accuracy of the space discretization. For a second-order TVD accurate scheme, as the one described in the previous section, a fivepoint space scheme is applied for each dimension. Therefore we have $s^{\prime}=2$. In addition, the number of nearest uncles $s$ depends on the multiresolution accuracy. 


\section{Conservative Flux Computation}

To illustrate the conservative flux computation, we first consider a $1 \mathrm{D}$ leaf $\Omega_{j+1,2 k+1}$ whose cousins in the right direction $\Omega_{j+1,2 k+2}$ and $\Omega_{j+1,2 k+3}$ are virtual. Therefore their father $\Omega_{j, k+1}$ is a leaf.

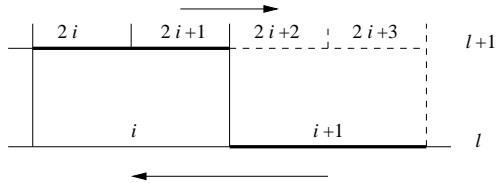

FIGURE 42. Ingoing and outgoing flux computation in 1D for two different levels.

As shown in Fig. 42, the outgoing flux from $\Omega_{j+1,2 k+1}$ in the right direction $F_{j+1,2 k+1 \rightarrow j+1,2 k+2}$ is not balanced with the outgoing flux from $\Omega_{j, k+1}$ in the left direction $F_{j, k+1 \rightarrow j, k}$. Of course, we could directly compute the outgoing fluxes from $\Omega_{j+1,2 k+1}$ to $\Omega_{j, k+1}$ in $1 \mathrm{D}$, but such a computation cannot be extended to higher dimensions, as we can see in Fig. 43.

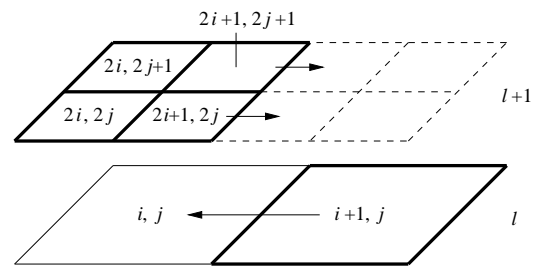

FIGURE 43. Ingoing and outgoing flux computation in 2D for two different levels.

So we decided to compute only the ones at the level $j+1$ and to set the ingoing flux on the leaf of level $j$ equal to the sum of the outgoing fluxes on the leaves of level $j+1$, i.e.

$$
F_{j, k, m \rightarrow j, k+1, m}=F_{j+1,2 k+1,2 m \rightarrow j+1,2 k+2,2 m}+F_{j+1,2 k+1,2 m+1 \rightarrow j+1,2 k+2,2 m+1}
$$

This choice ensures a strict conservativity in the flux computation between cells of different levels, without increasing significantly the number of costly flux evaluations.

\section{Error Analysis}

The global error between the cell-average values of the exact solution at the level $J$, denoted by $\bar{U}_{e x}^{J}$, and those of the multiresolution computation with a maximum level $J$, denoted by $\bar{U}_{M R}^{J}$, can be decomposed into two errors

$$
\left\|\bar{U}_{e x}^{J}-\bar{U}_{M R}^{J}\right\| \leq\left\|\bar{U}_{e x}^{J}-\bar{U}_{F V}^{J}\right\|+\left\|\bar{U}_{F V}^{J}-\bar{U}_{M R}^{J}\right\|
$$

where $\|\cdot\|$ denotes e.g. the $\mathcal{L}^{1}, \mathcal{L}^{2}$, or $\mathcal{L}^{\infty}$ norms. The first error on the right-hand side, called discretization error is the one of the finite volume scheme on the finest grid of level $J$. It can be bounded by

$$
\left\|\bar{U}_{e x}^{J}-\bar{U}_{F V}^{J}\right\| \leq C 2^{-\alpha J}, C>0
$$

where $\alpha$ is the convergence order of the finite volume scheme. In the present case, as we use second-order accurate schemes in time and space, we have $\alpha=2$.

As described in Section 2, Cohen et al. [17] showed that the second error, called perturbation error, can be controlled, if the discrete time evolution operator $\overline{\mathbf{E}}$ is a contraction in the chosen norm, there is no error in the 
flux computations, and the refinement strategy is done properly. In fact, under such conditions, if the details on a level $j$ are deleted when smaller than the prescribed tolerance $\epsilon_{j}$, which is set to

$$
\epsilon_{j}=2^{d(j-J)} \epsilon
$$

where $d$ is the space dimension, then the difference between finite volume solution on the finest grid and the solution obtained by multiresolution accumulates in time and satisfies

$$
\left\|\bar{U}_{F V}^{J}-\bar{U}_{M R}^{J}\right\| \leq C n \epsilon, C>0
$$

where $n$ denotes the number of time steps. At a fixed time $T=n \Delta t$, this leads to

$$
\left\|\bar{U}_{F V}^{J}-\bar{U}_{M R}^{J}\right\| \leq C \frac{T}{\Delta t} \epsilon, C>0 .
$$

For the linear convection-diffusion equation with the numerical scheme defined above, the time step $\Delta t$ must verify

$$
\Delta t \leq \frac{\Delta x^{2}}{4 \nu+c \Delta x}
$$

Denoting $X$ the size of the domain and $\Delta x$ the smallest space step, we have $\Delta x=X 2^{-J}$, from which we deduce that

$$
\Delta t=C \frac{\Delta x^{2}}{4 \nu+c \Delta x}=C \frac{X 2^{-2 J}}{4 \nu+c X 2^{-L}}, 0<C<1 .
$$

If we want the perturbation error to be of the same order as the discretization error, we need that

$$
\frac{\epsilon}{\Delta t} \propto 2^{-\alpha J}
$$

i.e.

$$
\epsilon 2^{2 J}\left(4 \nu+c X 2^{-J}\right) \propto 2^{-\alpha J}
$$

Defining the Peclet number $P e=c X \nu^{-1}$, the previous condition can be rewritten as

$$
\epsilon \propto \frac{2^{-(\alpha+1) J}}{P e+2^{J+2}} .
$$

For the inviscid case (i.e. $\nu=0$ or $P e \rightarrow+\infty)$, (32) is equivalent to the result obtained by Cohen et al. [17], i.e. $\epsilon \propto 2^{-(\alpha+1) J}$. In the numerical computations in Section 5 , the so-called reference tolerance will be set to

$$
\epsilon_{R}=C \frac{2^{-(\alpha+1) J}}{P e+2^{J+2}}
$$

To choose an acceptable value for the factor $C$, a series of computations with different tolerances will be necessary.

\subsection{Time Adaptive Strategies for the Multiresolution Scheme}

We consider three adaptive strategies in time to be used in combination with the FV-MR scheme, as illustrated in Figure 44. The CTS (Controlled Time Stepping) strategy is a classical strategy from ODE simulations, where the time step size selection is based on estimated local truncation errors. The main reason of controlling the error in the solution is to obtain an accurate and safe integration in the whole interval. When the estimated local error is smaller than a given tolerance, denoted by $\delta_{\text {desired, }}$, the algorithm increases the step size to make the integration more efficient. As shown in [33], it is possible to have stable simulations where the chosen time 
step eventually does not satisfy the CFL condition. In such case, the truncation error does increase, and thus the time step is automatically reduced by the CTS strategy. Local time stepping (LTS) refers to the time integration that uses scale-dependent time steps. Instead of using a single $\Delta t$ for the evolution on all cells, the time step is doubled when going to a coarser level with double spacing. Both adaptive time strategies can also be combined in a CTS/LTS scheme.

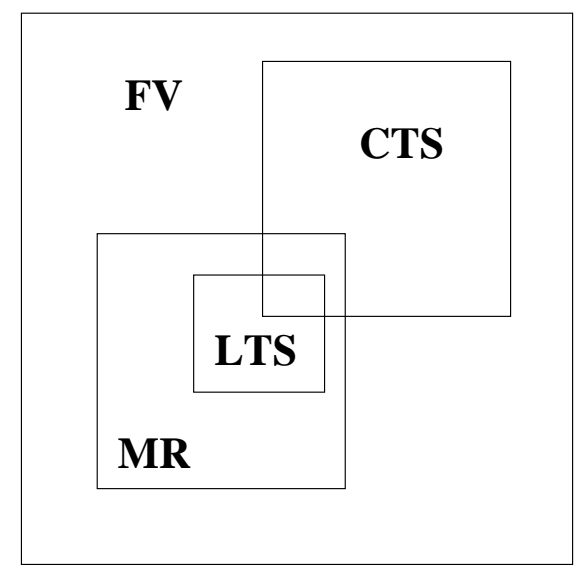

FIGURE 44. Formal illustration of space-time adaptive schemes.

\section{$M R / C T S$ Scheme}

For ODE simulations, instead of using a fixed time step $\Delta t$ chosen a priori, it can be advantageous to have a technique that automatically adjusts its size dynamically. The time step $\Delta t$ must be chosen sufficiently small to satisfy a required precision of the computed results, denoted by $\delta_{\text {desired }}$, but it must be sufficiently large to avoid unnecessary computational work. Typically, if $\bar{U}_{(1)}$ is the approximation of $\bar{U}(t+\Delta t)$ developments of the local truncation errors of the form

$$
\bar{U}(t+\Delta t)-\bar{U}_{(1)}=C \Delta t^{p+1}+O\left(\Delta t^{p+2}\right)
$$

could be used to find the step size required to attain a specified accuracy. However, since the leading constant $C$ is not known a priori, practical error estimates are necessary. To estimate the local truncation error, the idea is to apply two embedded ODE solvers, one with order $p$ and the other with order $p+1$. If $\bar{U}_{(2)}$ is the approximation of $\bar{U}(t+\Delta t)$ generated by the method of order $p+1$, then, for sufficiently small $\Delta t$ we have,

$$
\bar{U}_{(1)}-\bar{U}_{(2)} \approx C_{1} \Delta t^{p+1}-C_{2} \Delta t^{p+2} \approx C_{1} \Delta t^{p+1}
$$

This yields the estimate

$$
C_{1} \approx \frac{\bar{U}_{(1)}-\bar{U}_{(2)}}{\Delta t^{p+1}}
$$

Hence the step size required to maintain the local truncation error of the first scheme below $\delta_{\text {desired }}$ has the form $\Delta t_{n e w}=\Delta t \xi$, where

$$
\xi=\left[\frac{\delta_{\text {desired }}}{\left|\bar{U}_{(1)}-\bar{U}_{(2)}\right|}\right]^{1 /(p+1)} .
$$

If we want to prevent the time step of varying too abruptly or to be sure that $\Delta t_{n e w}$ will actually produce an error less than $\delta_{\text {desired }}$, some care is needed. In the present implementation, due to the low storage memory model we are using, we can not go back to the previous time step once the solution on the new time step is 
computed (as e.g. proposed by [41]). Hence we decided to limit the increase of the time step by introducing a safety factor $(\mathcal{S})$. The new time step $(\Delta t)_{\text {new }}$ is chosen such that

$$
-\frac{\mathcal{S}}{2} \leq \frac{(\Delta t)_{n e w}-(\Delta t)}{(\Delta t)} \leq \frac{\mathcal{S}}{2}
$$

Using a more stringent safety factor, the choice of non admissible time steps can be avoided. This method is also typically used for ordinary differential equations to avoid bad choices of the time step. A drawback of this limiter is that, in case that the initial time step is far from the ideal time step, CPU time could be wasted as the time step can not be increased sufficiently fast. To overcome this, we define $\mathcal{S}=\mathcal{S}(t)$ with an exponential decay during the first time steps, i.e,

$$
\mathcal{S}(t)=\left(\mathcal{S}_{0}-\mathcal{S}_{\text {min }}\right) \exp \left(-\frac{t}{\Delta t}\right)+\mathcal{S}_{\text {min }}
$$

The behavior of the limiter $\mathcal{S}(t)$ for $t=0$ is the maximal allowed variation $\mathcal{S}_{0}$ and, for $t \rightarrow \infty$, it is $\mathcal{S}_{\text {min }}$, where $\mathcal{S}_{\text {min }}<\mathcal{S}_{0}$. In the present paper we use $\mathcal{S}_{0}=0.1$ and $\mathcal{S}_{\text {min }}=0.01$ for all case studies presented. This means that we allow $10 \%$ of variation of the time step in the initial time step and after few iterations we allow only $1 \%$.

For the present applications, we adopt the Runge-Kutta Fehlberg 2(3), where the second order scheme is the one defined in (18). Given $\bar{U}^{*}$ computed as in (17), then the third order scheme computes one more stage to obtain

$$
\begin{aligned}
\bar{U}^{* *} & =\frac{1}{4}\left[3 \bar{U}^{n}+\bar{U}^{*}-\Delta t F\left(\bar{U}^{*}\right)\right], \\
\bar{U}^{n+1} & =\frac{1}{3}\left[\bar{U}^{n}+2 \bar{U}^{* *}-2 \Delta t F\left(\bar{U}^{* *}\right)\right] .
\end{aligned}
$$

In the MR/CTS setting, $\bar{U}$ is replaced by $\bar{U}_{M R}$, and $F(\bar{U})$ should be taken as $F_{M R}\left(\bar{U}_{M R}\right)$.

\section{$M R / L T S$ Scheme}

Assuming that $\Delta t$ is the time step used to evolve the cells on the finest scale level $L$, the main principle in the MR/LTS algorithm is to evolve the cells at lower level $0 \leq j<J$ with time step $2^{J-j} \Delta t$. Consequently, if $\bar{U}_{\mathrm{MR} / \mathrm{LTS}}^{n}$ represents the numerical solution at $t^{n}=n \Delta t$ on the adaptive grid $\Gamma^{n}=\Gamma_{J}^{n}$, then one complete time cycle of the local time stepping evolution operator evolves the solution from $t^{n}$ to $t^{n+2^{J}}$. This adaptive time strategy combined with the multiresolution scheme gives the fully adaptive time cycle

$$
\bar{U}_{\mathrm{MR} / \mathrm{LTS}}^{n+2^{J}}=\left(\mathbf{T}(\epsilon) \mathbf{E}_{\mathrm{MR} / \mathrm{LTS}} \mathbf{R}\right) \bar{U}_{\mathrm{MR} / \mathrm{LTS}}^{n},
$$

where $\mathbf{E}_{\mathrm{MR} / \mathrm{LTS}}$ denotes the evolution operator using the adaptive MR discretization combined with the scaledependent time stepping. Recall that $\mathbf{T}(\epsilon)$ corresponds to the coarsening (thresholding), and $\mathbf{R}$ to the refinement operations. For more details of the implementation of the MR/LTS scheme, we refer to [32].

\section{MR/CTS/LTS Scheme}

The MR/CTS/LTS scheme combines the two previous time adaptive strategies as follows:

- First, the MR/CTS strategy is applied just to determine a step size $\Delta t$ required to attain a specified accuracy with a global time stepping.

- Second, the MR/LTS cycle is computed using the obtained step size $\Delta t$ for the evolution of the cell averages in the finest scale level.

- Third another MR/CTS time step is done to adjust the time step, and so on. 
This technique hence allows to change the time step size during the time evolution to control the truncation error in time and to benefit from the local time stepping to reduce further the computational cost. Nevertheless, we should mention that the local time stepping implies large time cycles for many refinement levels. The size of the time cycle is increasing with the number of refinement levels. Therefore a wide range of adaptive scales in MR/CTS/LTS implies that the time step control becomes less efficient to adjust $\Delta t$ rapidly.

\section{Applications of the Finite Volume Multiresolution Scheme}

To illustrate the performance of the FV-MR methods described in the previous section, we present different numerical examples in one, two and three space dimensions In the following, the finite volume reference scheme $(\mathrm{FV})$, the adaptive multiresolution method (MR), the adaptive multiresolution method with local time stepping (MR/LTS) or/and controlled time stepping (MR/CTS, MR/CTS/LTS) are applied. First, we consider one and two-dimensional convection-diffusion equations. Then, we show computations for reaction-diffusion equations in one, two and three space dimensions, which correspond to a planar flame front, a flame ball initially stretched in one direction, and to a cellular instability inside a spherical flame initially stretched, respectively. Next, we consider the compressible Euler equations for a shock tube problem in one dimension, a 2D elliptical implosion, and a 2D Liu-Lax Riemann problem. For the former example, comparison results with the AMR method are shown. Finally, we present CVS computations of turbulent time-developing mixing layers.

\subsection{Convection-Diffusion Equations}

In One Space Dimension

We consider the linear convection-diffusion equation

$$
\frac{\partial u}{\partial t}+\frac{\partial u}{\partial x}=\nu \frac{\partial^{2} u}{\partial x^{2}},(x, t) \in[-1,1] \times[0,+\infty),
$$

with boundary values $u(-1, t)=1$ and $u(1, t)=0$. All the numerical solutions of this section are compared with the analytical solution (see e.g. [48])

$$
u_{e x}(x, t)=\frac{1}{2} \operatorname{erfc}\left(\frac{x-t}{2 \sqrt{\nu t}}\right)
$$

and the computations start at $u(x, 0)=u_{e x}(x, 0.1)$. For this example, we adopt the classical central second order FV scheme, with $\Delta t$ chosen as indicated in formula (31), taking $C=0.5$. Setting the Peclet number $P e=\frac{1}{\nu}$, the tolerance parameter $\epsilon$ is chosen according to the formula in [69]

$$
\epsilon=\epsilon_{J}=5 \cdot 10^{8} \frac{2^{-3 J}}{P e+2^{J+2}} .
$$

Using $P e=1000$, and $J=9$, the plots in Figure 45 represent the stability regions in the $R e \times \sigma$ plane, where $R e=\frac{\Delta x}{\nu}$ is the mesh Reynolds number, and $\sigma$ is the CFL parameter $\sigma=\frac{\Delta t}{\Delta x}$. for the FV, MR and MR/LTS methods obtained by checking the solution at $t=0.5$. For all schemes we performed computations for $\sigma$ ranging from 0.125 to 1.875 in steps of 0.125 and for $R e$ from 1 to 10 in steps of 1 . The stability region for the FV scheme depicted in Figure 45 (left) fits well with the theoretical one. As shown in Figure 45 (right), the stability domains for MR and MR/LTS schemes coincide. Moreover, they are quite similar to the one found for the FV scheme.

Table 3 presents a comparison of results for numerical solutions of the convection-diffusion equation at $t=0.5$ obtained with different methods and $J=9$ scales. The MR/LTS discretization error $\left\|u_{e x}-u_{L T S}\right\|$ can be decomposed into the FV discretization error $\left\|u_{e x}-u_{F V}\right\|$, the thresholding error $\left\|u_{F V}-u_{M R}\right\|$ and the local 


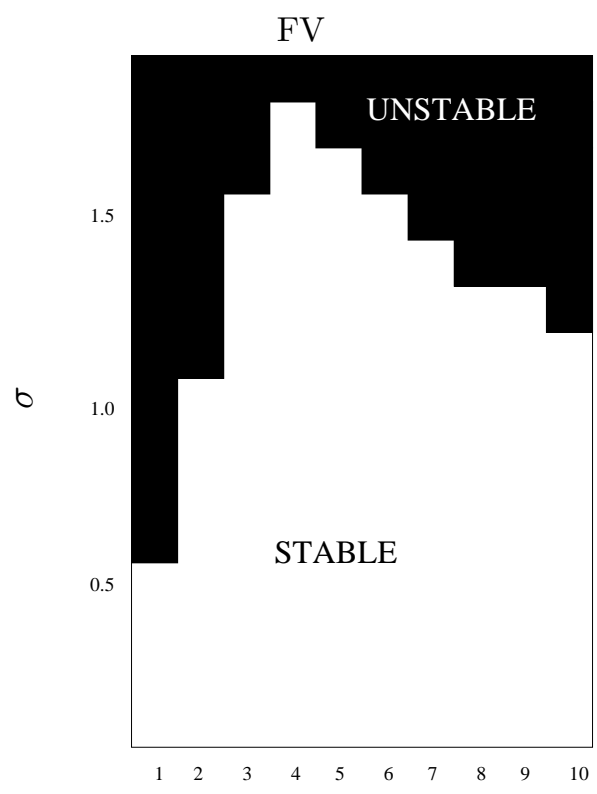

$\operatorname{Re}$

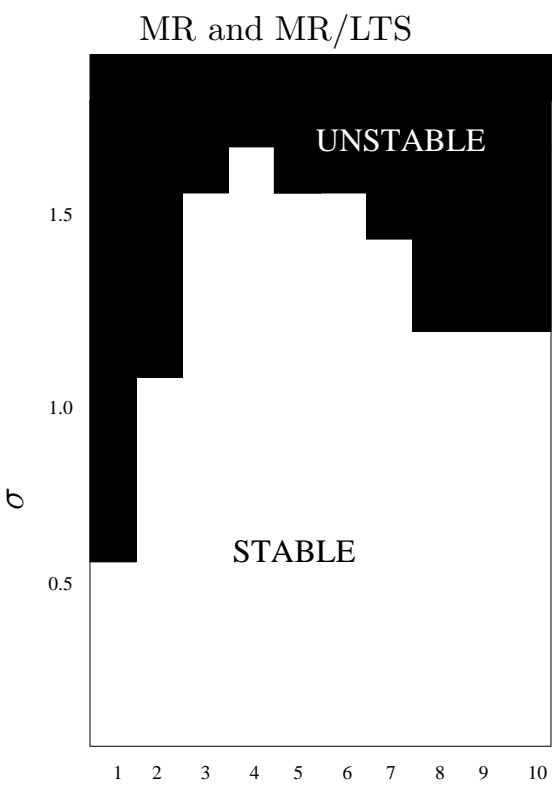

$\operatorname{Re}$

FIGURE 45. Numerically computed stability regions for the convection-diffusion equation using centred second order FV(left), MR and MR/LTS (right) schemes, with $J=9$.

TABLE 3. Contributions of the different errors for the $1 \mathrm{D}$ convection-diffusion simulation at $t=0.5$ for CFL parameter $\sigma=0.5, P e=1000, \epsilon=1.2 \times 10^{-3}$ and $J=9$.

\begin{tabular}{cccc}
\hline \hline Terms & $L_{1}$ norm & $L_{2}$ norm & $L_{\infty}$ norm \\
\hline$\left\|u_{e x}-u_{F V}\right\|$ & $1.9980 \cdot 10^{-3}$ & $2.9597 \cdot 10^{-4}$ & $1.4969 \cdot 10^{-2}$ \\
$\left\|u_{F V}-u_{M R}\right\|$ & $3.0991 \cdot 10^{-5}$ & $5.2205 \cdot 10^{-6}$ & $3.7992 \cdot 10^{-4}$ \\
$\left\|u_{M R}-u_{L T S}\right\|$ & $4.2655 \cdot 10^{-5}$ & $8.7415 \cdot 10^{-6}$ & $9.1523 \cdot 10^{-4}$ \\
$\left\|u_{e x}-u_{L T S}\right\|$ & $1.9890 \cdot 10^{-3}$ & $2.9403 \cdot 10^{-4}$ & $1.5501 \cdot 10^{-2}$ \\
\hline \hline
\end{tabular}

time stepping error $\left\|u_{M R}-u_{L T S}\right\|$. We can see that the the MR/LTS discretization error is almost identical with the FV discretization error, and the thresholding error is two orders of magnitude smaller.

Table 4 presents errors for different schemes versus the maximal scale $J$. We observe that MR and MR/LTS computations yield the same second order accuracy of the reference FV computation on a regular grid.

TABLE 4. Convection-diffusion: Error in $L_{\infty}$ and $L_{1}$ norm for FV, MR and MR/LTS methods obtained at $t=0.5$ for $\sigma=0.5, P e=1000$, and $J=9$ to 13 .

\begin{tabular}{c||cc|cc|cc}
\multicolumn{1}{c||}{$J$} & \multicolumn{2}{c|}{ FV } & \multicolumn{2}{c|}{ MR } & \multicolumn{2}{c}{ MR/LTS } \\
\cline { 2 - 7 } & $L_{1}$ norm & $L_{\infty}$ norm & $L_{1}$ norm & $L_{\infty}$ norm & $L_{1}$ norm & $L_{\infty}$ norm \\
\hline 9 & $1.1336 \cdot 10^{-2}$ & $4.7938 \cdot 10^{-4}$ & $1.14269 \cdot 10^{-2}$ & $2.3251 \cdot 10^{-3}$ & $1.1323 \cdot 10^{-2}$ & $2.3924 \cdot 10^{-3}$ \\
10 & $2.9642 \cdot 10^{-3}$ & $1.2467 \cdot 10^{-4}$ & $2.98725 \cdot 10^{-3}$ & $6.5649 \cdot 10^{-4}$ & $3.3521 \cdot 10^{-3}$ & $7.0108 \cdot 10^{-4}$ \\
11 & $7.5534 \cdot 10^{-4}$ & $3.1742 \cdot 10^{-5}$ & $7.57143 \cdot 10^{-4}$ & $1.8253 \cdot 10^{-4}$ & $9.9878 \cdot 10^{-4}$ & $2.2443 \cdot 10^{-4}$ \\
12 & $1.9016 \cdot 10^{-4}$ & $7.9903 \cdot 10^{-6}$ & $1.90555 \cdot 10^{-4}$ & $4.5800 \cdot 10^{-5}$ & $1.9151 \cdot 10^{-4}$ & $4.6019 \cdot 10^{-5}$ \\
13 & $4.7644 \cdot 10^{-5}$ & $2.0019 \cdot 10^{-6}$ & $4.76831 \cdot 10^{-5}$ & $1.1601 \cdot 10^{-5}$ & $4.8473 \cdot 10^{-5}$ & $1.1839 \cdot 10^{-5}$ \\
\hline \hline
\end{tabular}


The gain in CPU time of the MR/LTS computation with respect to the MR one is shown in Figure 46 (right) showing a gain of CPU time of the MR/LTS method, varying from $10 \%$ to $20 \%$, increasing with with the number of levels $9 \leq J \leq 12$. Moreover, it can be noticed that almost the same memory is required for both methods (Figure 46, left).
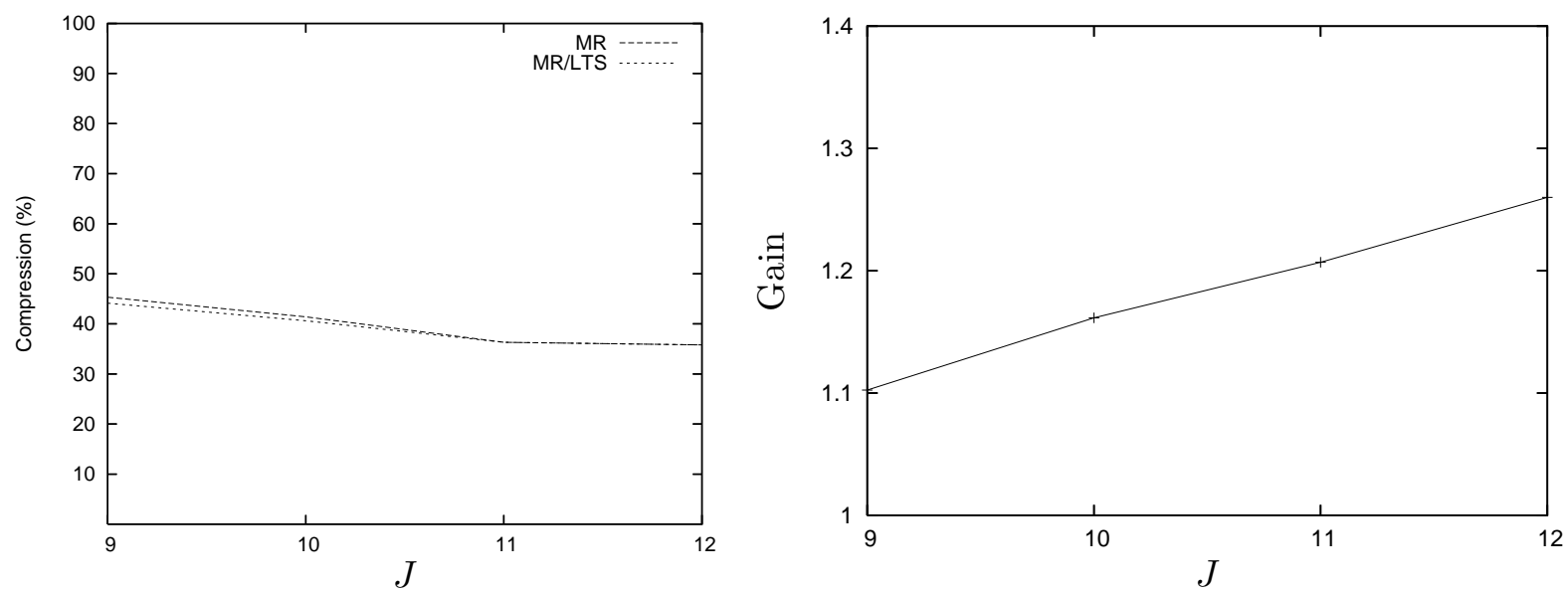

Figure 46. Memory compression for the convection-diffusion equation using both MR and MR/LTS methods (left) and gain in CPU time of the MR/LTS method in comparison with the MR method (right) for $\sigma=0.5, P e=1000, \epsilon=1.2 \times 10^{-3}$, and $J=9$ to 12 .

\section{In Two Space Dimensions}

In this part, we study the performances and check the second-order accuracy of the multiresolution scheme with the reference tolerance in the $2 \mathrm{D}$ case. Therefore we consider the dimensionless equation for $(x, y, t) \in$ $[-5,5]^{2} \times[0,+\infty)$

$$
\frac{\partial u}{\partial t}+\mathbf{V} \cdot \nabla u=\frac{1}{P e} \nabla^{2} u
$$

with the initial condition $u(x, y, 0)=u_{0}(x, y)$. Here we consider a convection in the $x$-direction, i.e. $\mathbf{V}=(1,0)^{T}$. For the initial condition $u_{0}(x, y)=\delta(x) \delta(y)$, where $\delta$ denotes the Dirac distribution, we have an analytic solution in an infinite domain

$$
u(x, y, t)=\frac{P e}{4 \pi t} e^{-P e \frac{(x-t)^{2}+y^{2}}{4 t}}
$$

For a Gaussian initial condition, we can change variables $(x \leftarrow x-\tau, t \leftarrow t-\tau$, where $\tau>0)$. Thus, given the initial condition

we get the analytic solution

$$
u_{0}(x, y)=\frac{P e}{4 \pi \tau} e^{-P e \frac{x^{2}+y^{2}}{4 \tau}}
$$

$$
u(x, y, t)=\frac{P e}{4 \pi(t+\tau)} e^{-P e \frac{(x-t)^{2}+y^{2}}{4(t+\tau)}}
$$

For the numerical computations, the boundaries are set far enough from the Gaussian bump, so that their influence can be considered as negligible, and $\Delta t=O\left(\Delta x^{2}\right.$, as in the one dimensional case.

The numerical solution of (38) for an initial Gaussian bump is represented in Fig. 47 for $P e=1000, \epsilon=\epsilon_{R}$, and $J=8$ scales, which represents a maximum of $\left(2^{8}\right)^{2}=256^{2}$ cells. In the figures where the corresponding meshes are plotted, each point represents a leaf. For the initial condition, we set $\tau=0.1$.

We observe the phenomenon of linear propagation of the 2D Gaussian bump in the $x$-direction. The diffusion effect is difficult to detect, but we can see that the radius of the smallest circle slightly decreases with time. 

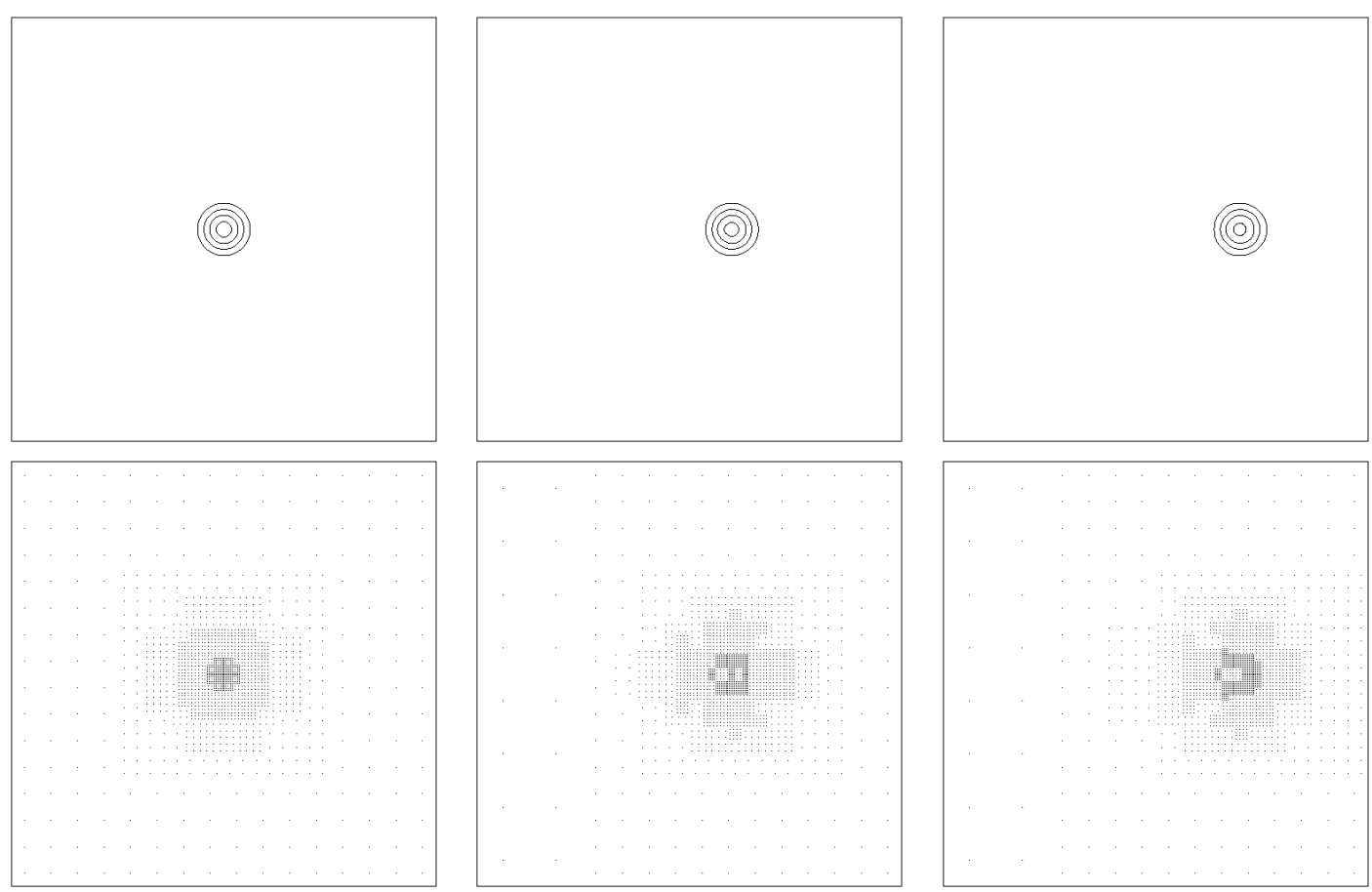

FIgURE 47. Isolines $u=0.3,0.5,0.7$, and 0.9 (top) and corresponding mesh(bottom) for the $2 \mathrm{D}$ convection-diffusion equation at $t=0$ (left), $t=1$ (middle), and $t=2$ (right).

We also remark that the adaptive mesh follows well the propagation. Nevertheless, although the mesh is well symmetric at the initial condition, it remains symmetric only on the two sides of the $x$-axis, whereas it is not in the other direction. This is due to the fact that the advection takes place in the $x$-direction.
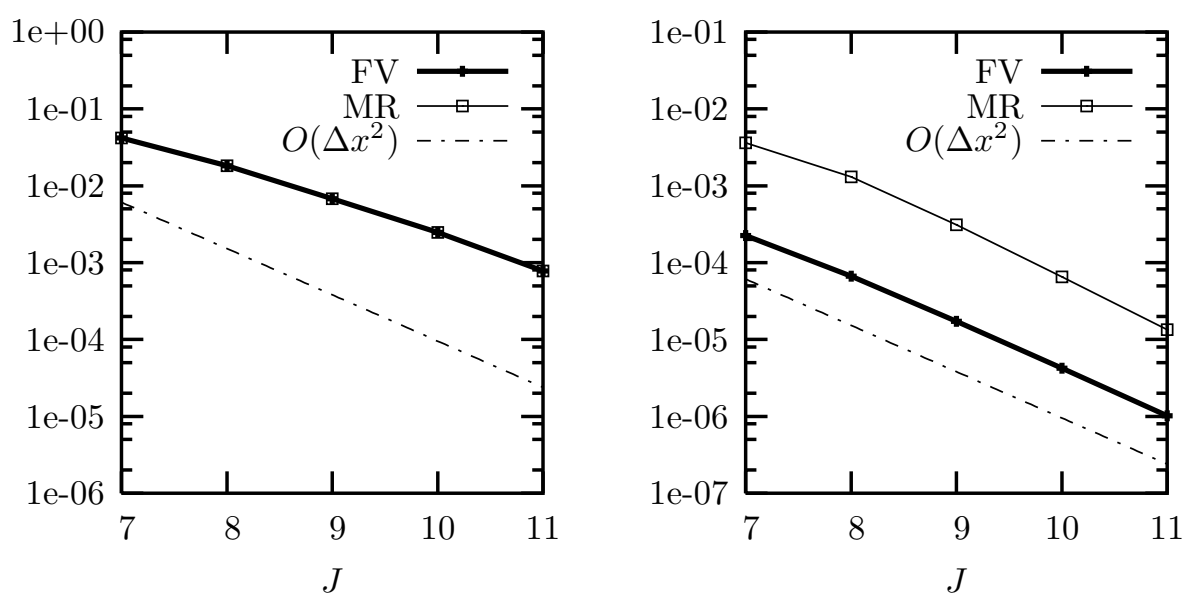

FiguRE 48. Errors $\left\|\bar{u}-\bar{u}_{e x}\right\|_{\infty}$ (left) and $\left\|\bar{u}-\bar{u}_{e x}\right\|_{1}$ (right) for different scales $J$ and the reference tolerance $\epsilon_{R}$ for the $2 \mathrm{D}$ convection-diffusion equation $P e=1000, t=0.5$. 
As the definition of the reference tolerance is independent of the space dimension, we use the same one as in the $1 \mathrm{D}$ case, i.e. $C=5.10^{8}$. We remark here that both CPU and memory compressions are low and stable with $J$ (around $15 \%$ for the CPU compression, $10 \%$ for the memory compression), while the corresponding errors confirm that the computations are well second-order accurate (Fig. 48). This time, as no discontinuity exists in the initial condition and as the equation is linear, no steep gradient exists, which explains that the same percentage of leaves is used whatever $J$, although more levels are used around the Gaussian bump.

\subsection{Reaction-Diffusion Equations}

Another prototype of a non-linear parabolic equation is the reaction-diffusion equation. Here the non-linearity is no more in the advective term, as e.g. for the viscous Burgers equation, but in the source term.

\section{In One Space Dimension}

In its dimensionless form, the reaction-diffusion equation in one space dimension, for $(x, t) \in[0,20] \times[0,+\infty)$ can be written as,

$$
\begin{aligned}
\frac{\partial u}{\partial t} & =\frac{\partial^{2} u}{\partial x^{2}}+S(u) \\
S(u) & =\frac{\beta^{2}}{2}(1-u) \exp \frac{\beta(1-u)}{\alpha(1-u)-1} .
\end{aligned}
$$

where $\alpha$ is the temperature ratio and $\beta$ is the dimensionless activation energy (Zeldovich number). We choose as initial condition

$$
u_{0}(x)=\left\{\begin{array}{lll}
1 & \text { if } & x \leq 1 \\
\exp (1-x) & \text { if } & x>1
\end{array}\right.
$$

This equation yields a model for a 1D premixed flame propagation where heat and mass diffusivities are equal. The function $u$ is the dimensionless temperature. It varies between 0 and 1 . The non-dimensional partial mass of the unburnt gas is $1-u$. We choose a Neumann condition at the left boundary and a Dirichlet condition at the right boundary.

$$
\begin{aligned}
& \frac{\partial u}{\partial x}(0, t)=0 \\
& u(20, t)=0
\end{aligned}
$$

For the numerical computation, the parameters are $\alpha=0.8$ and $\beta=10$. The dimensionless time goes from $t=0$ to $t=t_{f}=10$.

In Fig. 49, we observe the flame propagation in the $x$-direction. The highest level is reached in the region of the reaction zone, i.e. for $x \approx 10$. We can also notice that the multiresolution computation gives the same result as the finite volume one. We then compare the value of the flame velocity, defined by

$$
v_{f}=\int_{\Omega} S d x
$$

with the asymptotic value given in Peters and Warnatz [62]. We observe that the value of $v_{f}$ is approximately the same for finite volume and multiresolution computations, for the three different values of the tolerance. All these values are comparable with the asymptotic one. Hence we can conclude that the value $\epsilon=5.10^{-2}$ is well adapted.

\section{In Two Space Dimensions}

In this part, the $2 \mathrm{D}$ reaction-diffusion equation is solved for a flame ball initially stretched in one direction. As in the 1D case, heat and mass diffusivities are equal. This test-case was originally proposed in [42]. The resulting equation in the dimensionless form is

$$
\frac{\partial u}{\partial t}=\frac{\partial^{2} u}{\partial x^{2}}+\frac{\partial^{2} u}{\partial y^{2}}+S(u)
$$



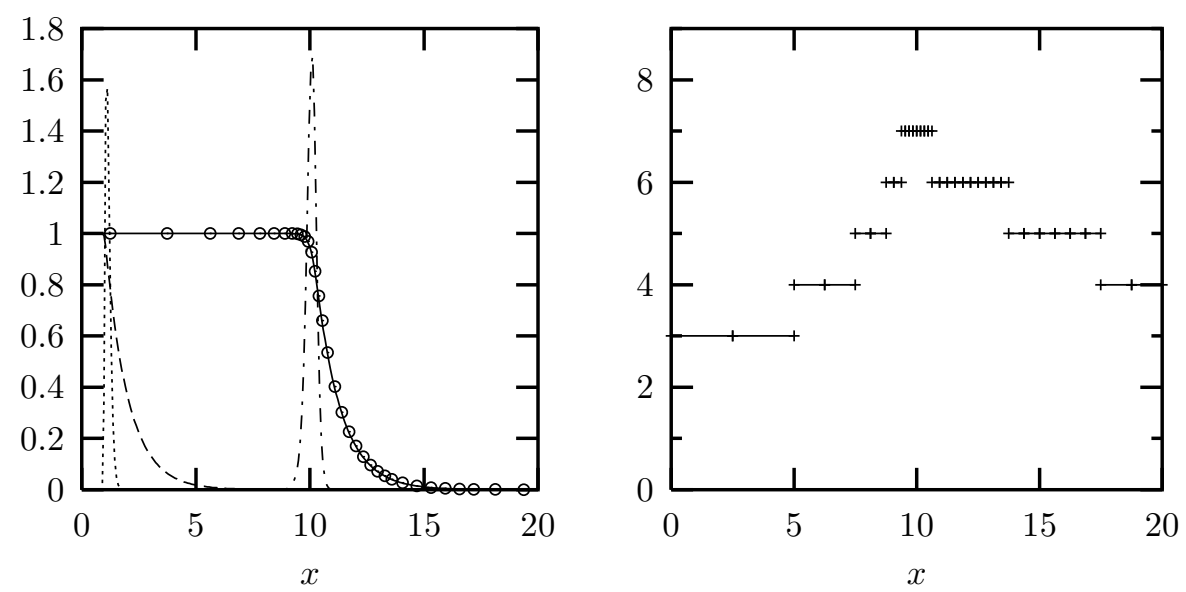

FigurE 49. Left: Initial condition for $u$ (dashed) and $S(u)$ (dotted), solution by finite volume method for $u$ (plain), solution by multiresolution method for $u$ (circles) and $S(u)$ (dash-dotted) at $t=10$ for the reaction-diffusion equation, $\alpha=0.8, \beta=10, J=8, \epsilon=5.10^{-2}$. Right: corresponding tree structure at $t=10$.

TABLE 5. Flame velocity, CPU and memory compression for finite volume and multiresolution methods.

\begin{tabular}{lrrr}
\hline Method & $v_{f}$ & $\%$ CPU & $\%$ Mem \\
\hline FV & 0.916 & $100.0 \%$ & $100.0 \%$ \\
MR $\epsilon=5.10^{-2}$ & 0.917 & $36.0 \%$ & $32.6 \%$ \\
MR $\epsilon=10^{-2}$ & 0.916 & $54.2 \%$ & $47.1 \%$ \\
MR $\epsilon=10^{-3}$ & 0.916 & $79.0 \%$ & $67.2 \%$ \\
\hline Asymptotic & 0.908 & & \\
\hline
\end{tabular}

where $(x, y, t) \in[-20,20]^{2} \times[0,+\infty)$ and $S(u)$ verifies (42). The initial condition is $u(x, y, 0)=u_{0}(r)$, where $u_{0}$ verifies (43) and $r^{2}=x^{2}+y^{2}$. We perturb the circular initial condition by stretching the circle in one direction and applying a rotation. Therefore, we have

$$
r=\sqrt{\frac{X^{2}}{a^{2}}+\frac{Y^{2}}{b^{2}}}
$$

where

$$
\left\{\begin{array}{l}
X=x \cos \theta+y \sin \theta, \\
Y=-x \sin \theta+y \cos \theta .
\end{array}\right.
$$

We consider that the reaction takes place in a closed box with adiabatic walls, and hence we choose Neumann conditions on the boundary, i.e.

$$
\left.\frac{\partial u}{\partial n}\right|_{\partial \Omega}=0 .
$$

The parameters are the Zeldovich number $\beta=10$, and the temperature ratio $\alpha=0.8$. The aspect ratio of the ellipse is given by $a=2, b=1$, and the rotation angle is $\theta=-\frac{\pi}{6}$. The elapsed time is $t=10$. For the multiresolution computation, the tolerance is set to $\epsilon=5 \cdot 10^{-2}$, like in the 1D case.

As in Fröhlich and Schneider [42], we observe a relaxation of the elliptic flame towards a circularly symmetric structure which is then growing in space. The finest resolution would correspond to $\left(2^{8}\right)^{2}=256^{2}$ cells. On average we only use 6763 out of $256^{2}=65536$ control volumes, which yields a memory compression of $10.3 \%$. 

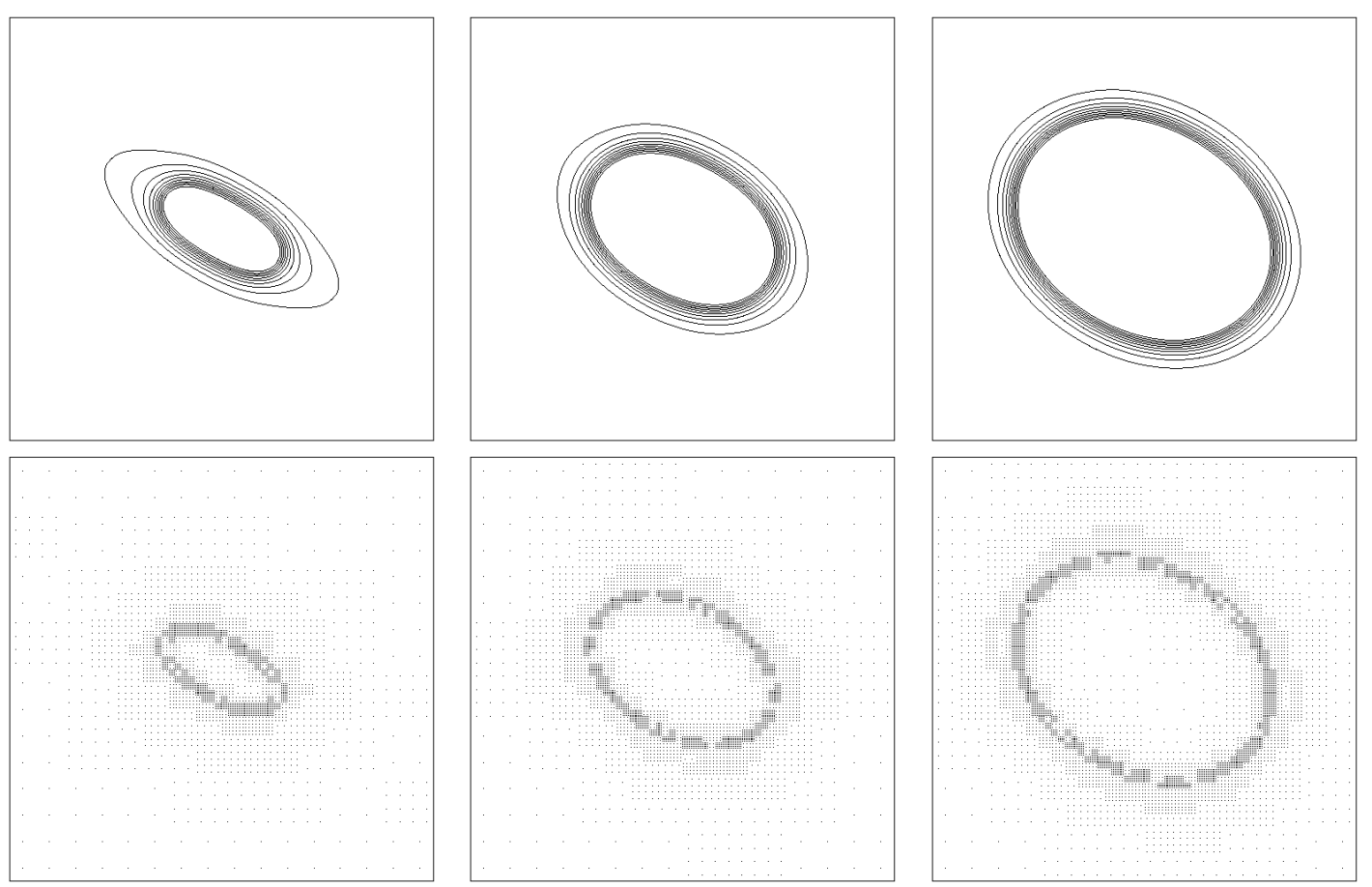

Figure 50. Isolines $u=0.1$ to 1 and corresponding mesh at $t=2$ (left), $t=6$ (middle), and $t=10$ (right) for the $2 \mathrm{D}$ reaction-diffusion equation.

Comparing the elapsed CPU time with the one obtained by the same finite volume scheme on the finest grid, we get a CPU compression of $19.9 \%$.

\section{In Three Space Dimensions}

The previous equation is now extended to three dimensions, in order to study the evolution of a 3D flame ball initially stretched in one direction, for equal heat and mass diffusivities. Therefore we consider the dimensionless equation for $(x, y, z, t) \in[-20,20]^{3} \times[0,+\infty)$

$$
\frac{\partial u}{\partial t}=\frac{\partial^{2} u}{\partial x^{2}}+\frac{\partial^{2} u}{\partial y^{2}}+\frac{\partial^{2} u}{\partial z^{2}}+S(u)
$$

where $S(u)$ verifies (42). The initial condition is now $u(x, y, z, 0)=u_{0}(r)$, where $u_{0}$ verifies $(43)$ and $r^{2}=$ $x^{2}+y^{2}+z^{2}$. The spherical initial condition is stretched in one direction and the same rotation is applied as previously. Therefore we have

where

$$
r=\sqrt{\frac{X^{2}}{a^{2}}+\frac{Y^{2}}{b^{2}}+\frac{Z^{2}}{c^{2}}},
$$

$$
\left\{\begin{array}{l}
X=x \cos \theta+y \sin \theta \\
Y=-x \sin \theta+y \cos \theta \\
Z=z .
\end{array}\right.
$$

As in the $2 \mathrm{D}$ case, we consider that the reaction takes place in a closed box with adiabatic walls, which means that

$$
\left.\frac{\partial u}{\partial n}\right|_{\partial \Omega}=0
$$


The Zeldovich number and the temperature ratio are the same as in the $2 \mathrm{D}$ case. The aspect ratio of the ellipsoid is given by $a=2, b=1, c=1$, and the rotation angle is $\theta=-\frac{\pi}{3}$. The elapsed time is $t=12$. For the multiresolution computation, the tolerance is set to $\epsilon=5 \cdot 10^{-2}$.
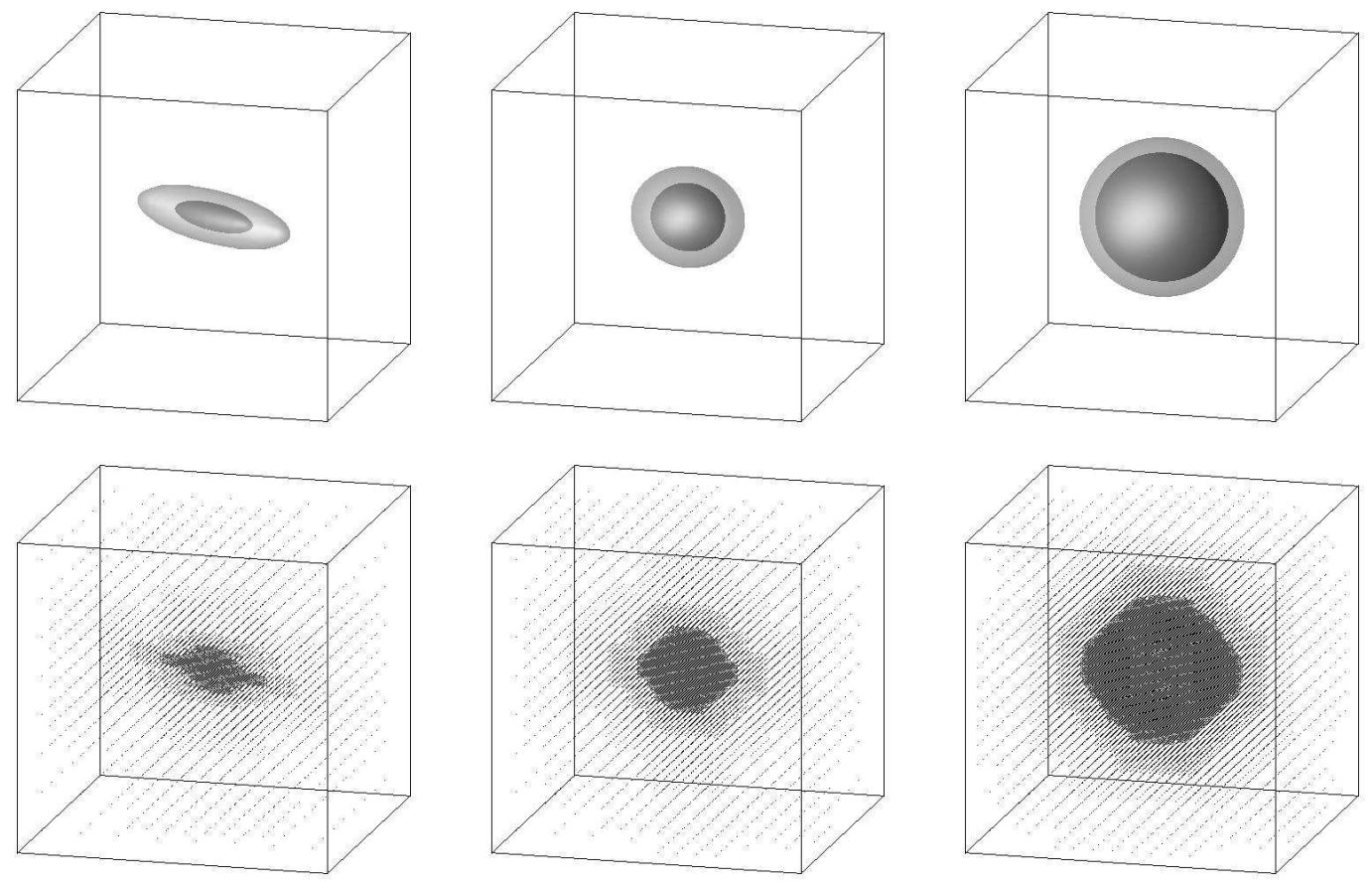

Figure 51. Isosurfaces $u=0.5$ (black), 0.1 (gray) and corresponding mesh at $t=0$ (left), $t=6$ (middle), and $t=12$ (right) for the 3D reaction-diffusion equation.

We observe, as in the 2D case, a relaxation of the ellipsoidal flame towards a spherically symmetric structure which is then growing in space, which shows that the perturbation is not amplified. The finest resolution would correspond to $\left(2^{7}\right)^{3}=128^{3}$ cells. On average we only use 39636 out of $128^{3}=2097152$ control volumes, which yields a memory compression of $1.89 \%$. Comparing the elapsed CPU time with the one obtained by the same finite volume scheme on the finest grid, we get a CPU compression of $7.64 \%$. For splitting flames, we refer to Roussel and Schneider [65].

\subsection{Euler Equations}

\section{D: Lax Test-Case}

The compressible Euler equations in 1D could be written in the following form,

$$
\frac{\partial Q}{\partial t}+\frac{\partial F}{\partial x}=0
$$

with,

$$
Q=\left(\begin{array}{c}
\rho \\
\rho v \\
\rho e
\end{array}\right) \quad \text { and } \quad F=\left(\begin{array}{c}
\rho v \\
\rho u^{2}+p \\
(\rho e+p) v
\end{array}\right)
$$

where $\rho=\rho(x, t)$ is the density, $v=v(x, t)$ is the velocity in the $x$-direction, $e=e(x, t)$ is the energy per unit of mass and $p=p(x, t)$ is the pressure. 
The system is completed by the equation of state for an ideal gas

$$
p=\rho r T=(\gamma-1) \rho\left(e-\frac{u^{2}}{2}\right)
$$

where $T=T(x, t)$ is the temperature, $\gamma=\gamma(x, t)$ is the specific heat ratio and $r$ is the universal gas constant. In dimensionless form, we obtain the same system of equations, but the equation of state becomes

$$
p=\frac{\rho T}{\gamma M a^{2}}
$$

where $M a$ denotes the Mach number.

We compute the Lax test-case in the computational domain $\Omega=[-1,1]$. The initial condition is given by

$$
Q(x, t=0)=\left\{\begin{array}{l}
\left(\begin{array}{c}
0.445 \\
0.311 \\
8.928
\end{array}\right) \\
\left(\begin{array}{c}
0.5 \\
0 \\
1.4275
\end{array}\right) \text { for } x<0,
\end{array}\right.
$$

Neumann boundary conditions are applied on both sides of the domain. The physical parameters are $M a=1$ and $\gamma=1.4$ and the computations are performed until physical time $t=0.32$. In this case, a rarefaction wave is moving to the left when $t>0$, and a contact discontinuity and a shock wave are propagating to the right (cf. Figure 52, bottom, left). More details on this test-case and its exact solution can be found in [54,83].

The time step $\Delta t$ used in the FV, MR, MR/LTS computations is $\Delta t=4.343 \times 10^{-4}$ and the threshold is chosen as $\epsilon=5 \times 10^{-3}$ for the MR methods. For the controlled time step experiments MR/CTS and $\mathrm{MR} / \mathrm{CTS} / \mathrm{LTS}$ we use a required precision $\delta_{\text {desired }}=0.07$. For MR/CTS we start with $\sigma=\Delta t / \Delta x=0.7$ and we find a minimum time step $4.343 \times 10^{-4}$. For MR/CTS/LTS with $\sigma=0.5$ we find $\Delta t=4.786 \times 10^{-4}$. Therefore MR/CTS/LTS uses a larger time step than MR/CTS to compute the solution.

In Figure 52 we observe that the numerical solutions fit well the exact one. On the contact region all numerical solutions are a slightly smoother, however they detect well the shock region present in the exact solution. As expected, the highest level on the adaptive grids is reached in the vicinity of the discontinuities of the functions and their first derivatives (Figure 52). Let us remark that, for both MR/CTS and MR/CTS/LTS methods, the time step is converging towards a value corresponding to $\sigma=0.45$.

In Table 6, we separately compare second order and third order methods for the time integration. For the $L^{1}$ error of density, shown in Table 6, the MR/CTS/LTS and MR/CTS present the best results of the MR methods. The kinetic energy of MR, MR/LTS, MR/CTS and MR/CTS/LTS, defined by $E=\frac{1}{2} \sum_{i \in \Lambda} \rho_{i}\left|\vec{v}_{i}\right|^{2} h_{x_{i}}$, is compared with the kinetic energy of the exact solution. In all the cases, it differs not more than $0.1 \%$, as one can observe in Table 6 . For the experiments with $J=10$, the best CPU performance is given by MR/CTS/LTS using $30.30 \%$ of FV RK3 CPU time (see Table 6). Moreover, the MR/LTS (RK2) scheme is faster than MR/RK2 one without loosing too much accuracy, and the MR/CTS/LTS scheme yields smaller errors than the MR/LTS one, for approximately the same CPU time. Concerning the memory compression we find approximately the same result whatever the chosen MR method. It proves that the space adaption is largely independent from the chosen time adaptive method. Nevertheless, the LTS methods require a little more memory due to the fact that a leaf cell can only be removed when the time cycle is finish on its level. 

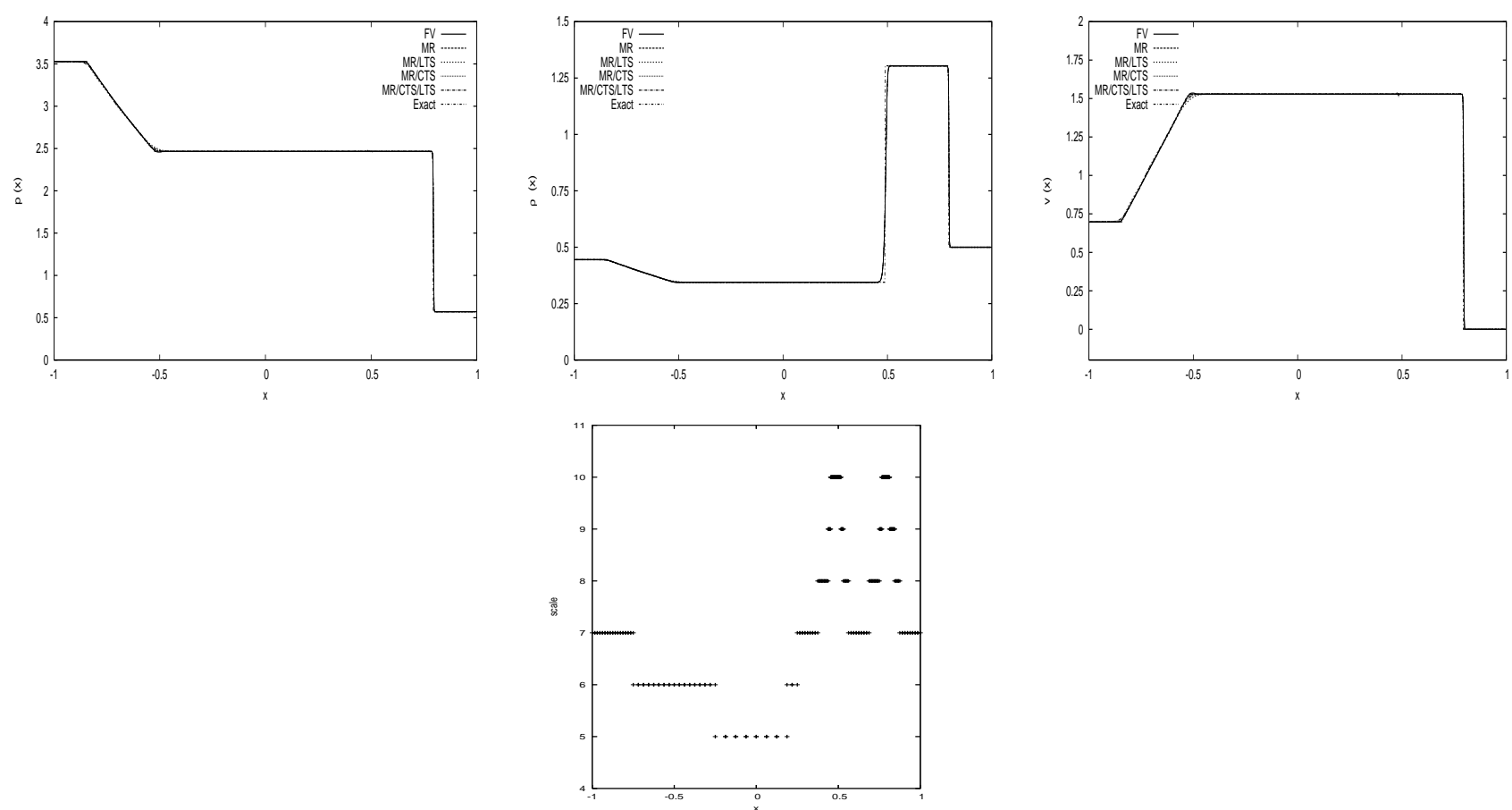

FiguRE 52. Exact and numerical solutions of Lax test-case for the FV and the MR methods at $t=0.32$ with $J=10$ and $\epsilon=5 \times 10^{-3}$. Top: pressure, density, velocity. Bottom: adaptive grid.

TABLE 6. Comparison for the errors and speed-up of the numerical solutions for the 1D Euler equations using the Lax test-case at time $t=0.32$ with $J=10$ levels.

\begin{tabular}{lrrrr}
\hline \hline Method & $L^{1}-$ error & E error & CPU & \\
& on $\rho$ & $(\%)$ & Time (\%) & Memory (\%) \\
\hline FV, RK2 & 0.0086 & 0.13 & 100.00 & 100.00 \\
MR, RK2 & 0.0087 & 0.13 & 39.13 & 44.69 \\
MR/LTS, RK2 & 0.0091 & 0.14 & 30.44 & 44.51 \\
\hline FV, RK3 & 0.0085 & 0.11 & 100.00 & 100.00 \\
MR, RK3 & 0.0085 & 0.11 & 36.36 & 44.69 \\
MR/CTS, RK2(3) & 0.0084 & 0.16 & 36.36 & 45.01 \\
MR/CTS/LTS, RK2(3) & 0.0083 & 0.08 & 30.30 & 47.49 \\
\hline \hline
\end{tabular}

\section{D: Elliptical Implosion}

As 2D test-case, we study an inviscid implosion phenomenon. In a square box, an elliptic diaphragm separates two regions which contain the same gas, but with different conditions of pressure and temperature. Inside the diaphragm, the pressure is lower than outside. In both regions, the gas is at rest, i.e., the initial velocity vanishes everywhere. On the boundary we impose zero flux boundary conditions.

At $t=0$, the diaphragm is broken. A shock wave and a contact discontinuity are moving towards the center, while a rarefaction wave is moving in the opposite direction.

To obtain the 2D Euler equations in its conservative form the variables are $Q=\left(\rho, \rho v_{1}, \rho v_{2}, \rho e\right)^{T}$ which corresponds to the vector of conserved variables. Here $\rho$ is the fluid density, $v_{1}, v_{2}$ are the velocity components 
in $x$ and $y$ direction and $e$ is the energy per unit of mass. The flux function $f=\left(f_{1}, f_{2}\right)^{T}$ is given by

$$
f_{1}=\left(\begin{array}{c}
\rho v_{1} \\
\rho v_{2}^{2}+p \\
\rho v_{1} v_{2} \\
(\rho e+p) v_{1}
\end{array}\right), \quad f_{2}=\left(\begin{array}{c}
\rho v_{2} \\
\rho v_{1} v_{2} \\
\rho v_{2}^{2}+p \\
(\rho e+p) v_{2}
\end{array}\right)
$$

where the pressure $p$ satisfies

$$
p=(\gamma-1) \rho\left(e-\frac{1}{2}\left(v_{1}^{2}+v_{2}^{2}\right)\right)
$$

and $\gamma=1.4$ denotes the specific heat ratio.

The initial conditions are

$$
\begin{aligned}
& \rho(r, 0)=\left\{\begin{array}{ccc}
1 & \text { if } & r \leq r_{0} \\
0.125 & \text { if } & r>r_{0},
\end{array}\right. \\
& \rho e(r, 0)=\left\{\begin{array}{ccc}
2.5 & \text { if } & r \leq r_{0} \\
0.25 & \text { if } & r>r_{0},
\end{array}\right.
\end{aligned}
$$

$v_{1}=v_{2}=0$ and $r_{0}$ denotes the initial radius.

This initial condition is stretched in one direction and a rotation is applied. The radius thus becomes

$$
r=\sqrt{\frac{X^{2}}{a^{2}}+\frac{Y^{2}}{b^{2}}}
$$

with the new coordinates

$$
\begin{aligned}
& X=x \cos \theta-y \sin \theta \\
& Y=-x \sin \theta+y \cos \theta
\end{aligned}
$$

Figure 53 (left) displays the initial density field with $a=1 / 3, b=1, \theta=-\pi / 3, r_{0}=1$, computational domain $\Omega=[-2,2]^{2}, J=9$ and $\epsilon=10^{-2}$. Figure 53 (right) corresponds to the respective adaptive grid.
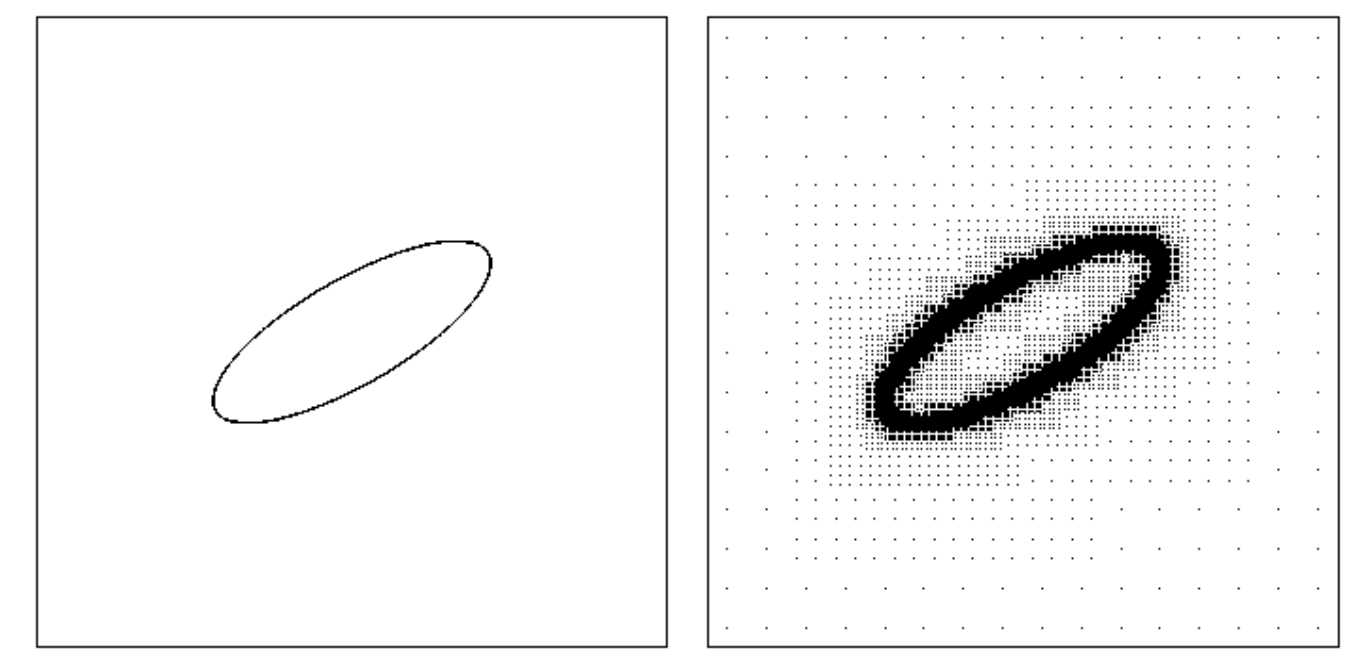

FIGURE 53. Initial condition of the 2D Euler equations: density contours and corresponding adaptive grid for $J=9$. 
Figure 54 displays the density field at $t=0.4$ computed with the FV RK3 and the MR RK3, MR/LTS RK2 and MR/CTS RK 2(3) methods. In Figure 55, the MR/CTS/LTS solution is plotted, together with the corresponding adaptive grid. We observe that all solutions match well the FV RK3 computation on the regular finest grid and that the adaptive grid tracks well the discontinuity and the steep gradients of the solution.
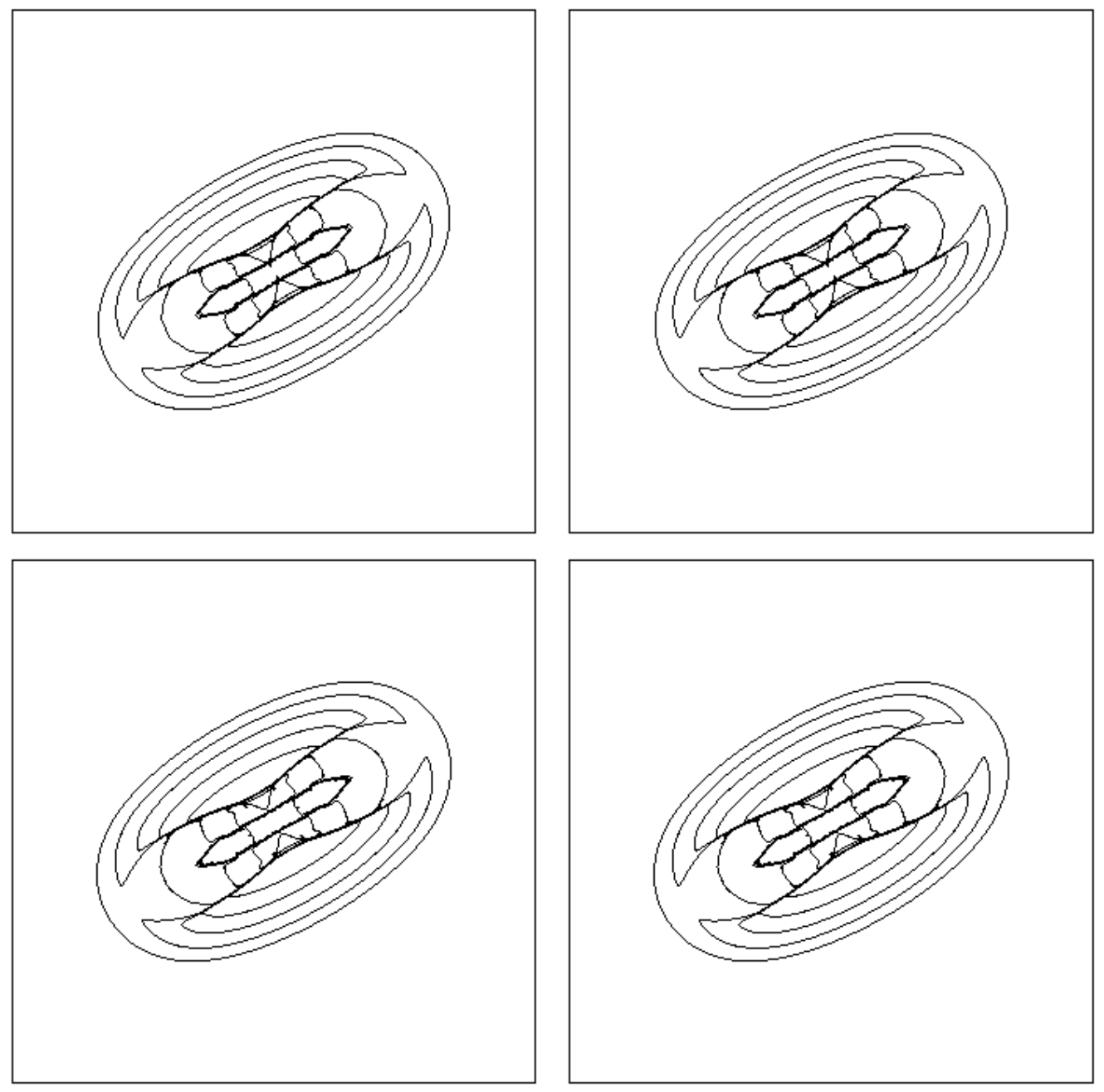

Figure 54. 2D Euler equations: density contours at $t=0.4, J=9$, for the FV (top left), MR (top right), MR/CTS (bottom left), and MR/LTS (bottom right) methods, $\epsilon=2 \cdot 10^{-3}$.

Density cuts are shown in Figure 56. To check for grid convergence we also superpose results of a FV computation using one additional level, i.e. $J=10$. We find a rather good agreement between both $\mathrm{FV}$ computations and hence can conclude that $J=9$ levels yield a sufficient solution for this problem. Concerning the different adaptive MR computations we also find a good agreement, which is further confirmed by the zoom (Fig. 56, right) into the central region of the computational domain.

In Table 7 we compare the computational efficiency and the precision of the different numerical methods using either second or third order time integration schemes. The reference computations are given by the FV scheme on the finest regular grid. For the memory compression we find for all adaptive schemes almost equivalent results, only about $26 \%$ of the memory required for the FV computations is used. The best precision of the adaptive 

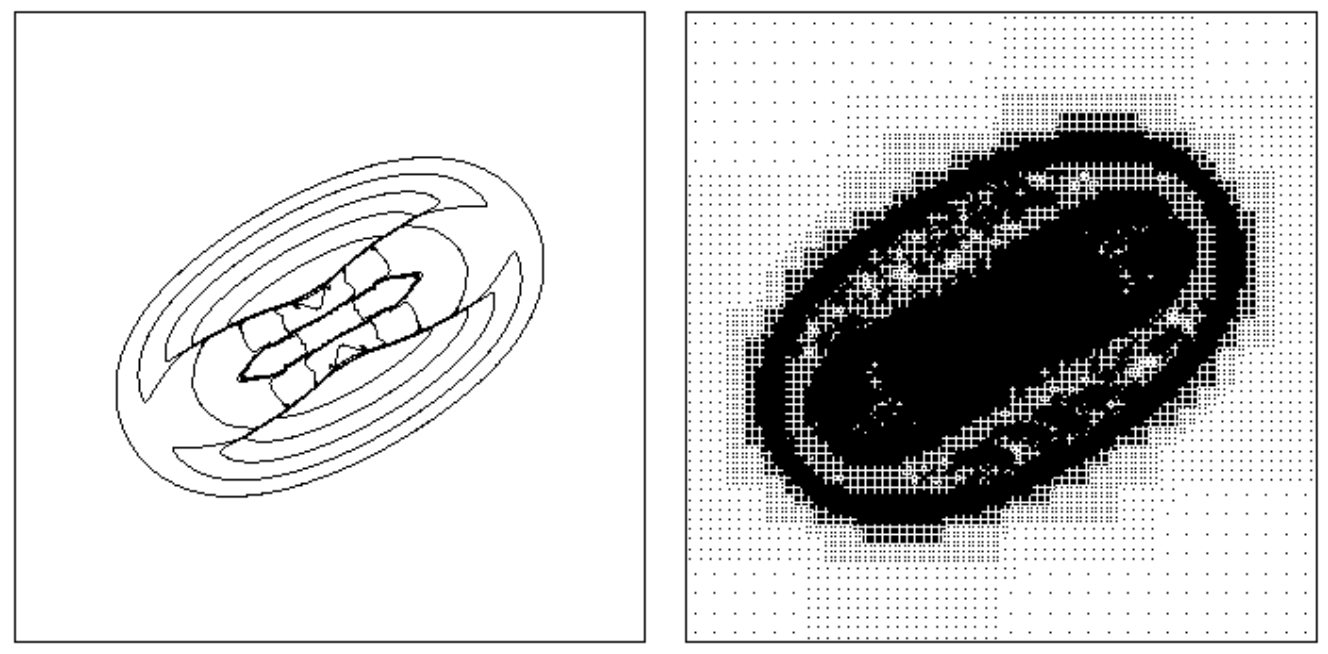

Figure 55. 2D Euler equations: density contours at $t=0.4, L=9$, for the MR/CTS/LTS method (left), and corresponding adaptive grid (right), $\epsilon=2 \cdot 10^{-3}$.
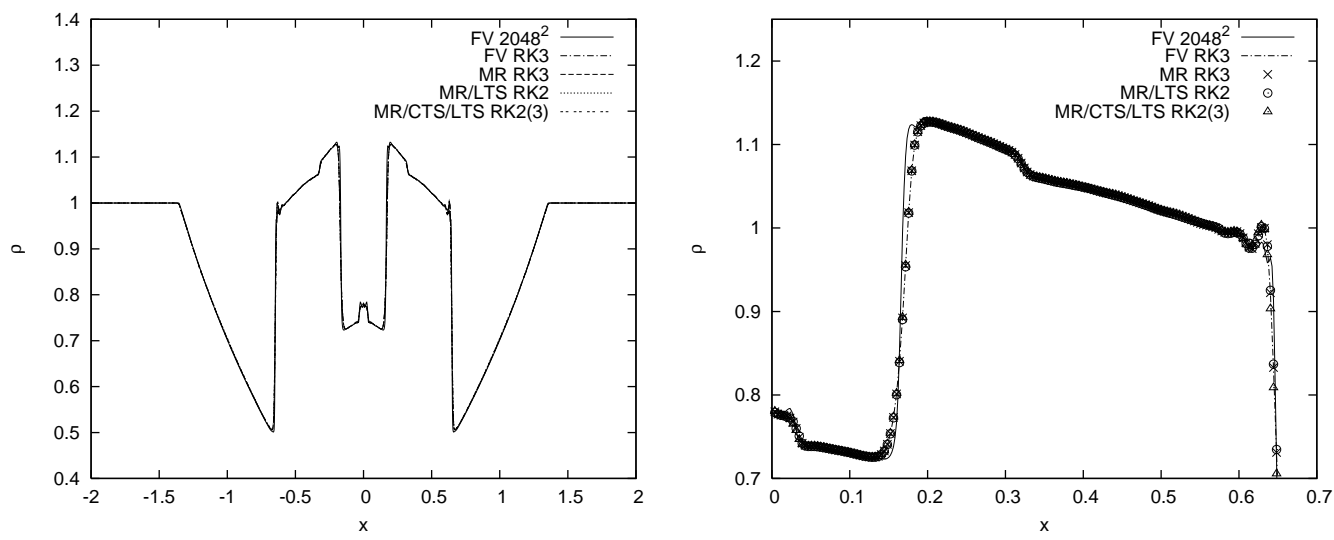

Figure 56. 2D Euler equations: density contours on the line $y=0$ for $t=0.4, J=9$ and $\epsilon=2 \cdot 10^{-3}$ for the different methods (left). Corresponding zoom of the central region $[-0.7,0.7]$ (right).

computation is obtained with the MR/CTS method followed by the two MR/RK2 and MR/RK3 methods. The MR/LTS computations show slightly larger errors due to the addition interpolation step. Concerning the speed up of the adaptive schemes. Table 7 shows that the MR/CTS/LTS method is 1.5 times faster than the MR/RK3 method. The latter scheme is already 5.5 faster than the FV/RK3 reference computation, which nicely illustrates the additional speed of adaptive and local time stepping.

\section{D: Liu-Lax Riemann Problem}

The case study chosen here is a typical Riemann problem for 2D gas dynamics treated e.g. in [52], and initially discussed in $[75,84]$. The initial data are constant in each quadrant (Figure 57), and the values are given in Table 8. This test case corresponds to the configuration \#5 in [52]. This classical test case only involves contact discontinuities and generates motion in opposite directions. 
TABLE 7. Comparison for the numerical solutions of the 2D Euler equations for $t=0.4, J=9$ and $\epsilon=2 \cdot 10^{-3}$. The reference FV kinetic energy is $E=23,18$ for RK2 and $E=23.91$ for RK3

\begin{tabular}{l|rrr}
\hline \hline Method & $\begin{array}{r}\text { E error } \\
(\%)\end{array}$ & Time (\%) & Memory (\%) \\
\hline FV/RK2 (ref.) & 0.00 & 100.00 & 100.00 \\
MR/RK2 & 0.08 & 28.71 & 26.55 \\
MR/LTS, RK2 & 0.26 & 28.76 & 26.26 \\
\hline FV/RK3 (ref.) & 0.00 & 100.00 & 100.00 \\
MR/RK3 & 0.07 & 27.21 & 26.60 \\
MR/CTS, RK2(3) & 0.01 & 24.33 & 26.79 \\
MR/CTS/LTS,RK2(3) & 0.15 & 17.96 & 26.17 \\
\hline \hline
\end{tabular}

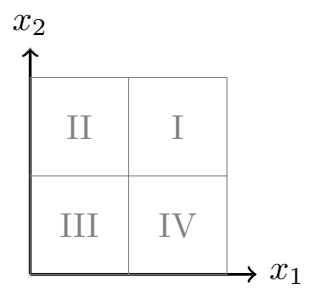

FIGURE 57. Sketch of the initial condition

TABLE 8. Initial Values for the Lax-Liu configuration \#5 [52]

\begin{tabular}{lcccc}
\hline \hline \multicolumn{1}{c}{ Variables } & \multicolumn{4}{c}{ Domain position } \\
& I & II & III & IV \\
\hline Density $(\rho)$ & 1.00 & 2.00 & 1.00 & 3.00 \\
Pressure $(p)$ & 1.00 & 1.00 & 1.00 & 1.00 \\
Velocity component $\left(v_{1}\right)$ & -0.75 & -0.75 & 0.75 & 0.75 \\
Velocity component $\left(v_{2}\right)$ & -0.50 & 0.50 & 0.50 & -0.50 \\
\hline
\end{tabular}

For this problem, as described in [27], we also present results of AMR simulations using thr AMROC code. For details on the AMROC solver, we refer the reader to [24-26] or tho the contribution by R. Deiterding in the present volume [23].

Both MR and AMR mesh refinement methods use AUSM-type numerical flux functions with comparable second-order accurate reconstruction and limiting. A series of computations are performed with CFL number 0.45 , and maximal level $J=8,9$ and 10 , respectively corresponding to $256 \times 256,512 \times 512$, and $1024 \times 1024$ cells on the finest uniform grid.The basic coarse grid for the AMR methods, which follows a patch-oriented refinement approach, is a $128 \times 128$ grid. As refinement indicators, scaled gradient criteria is adopted with threshold coefficients $\epsilon_{r} h o=\epsilon_{p}=0.05$ for density and pressure. Adaptive computations are performed first without, then with a local time stepping for both AMR and MR schemes.

The goal of an adaptive computation is to obtain the solution with a significant gain in CPU time and memory, while preserving the accuracy of the corresponding FV scheme on the regular finest grid. To assess the quality of an adaptive simulation, the discrete $L_{1}$-error is computed, using as reference the FV solution with the same space-time schemes on a $2048 \times 2048$ uniform grid. Figure 59 shows the isolines of density for 

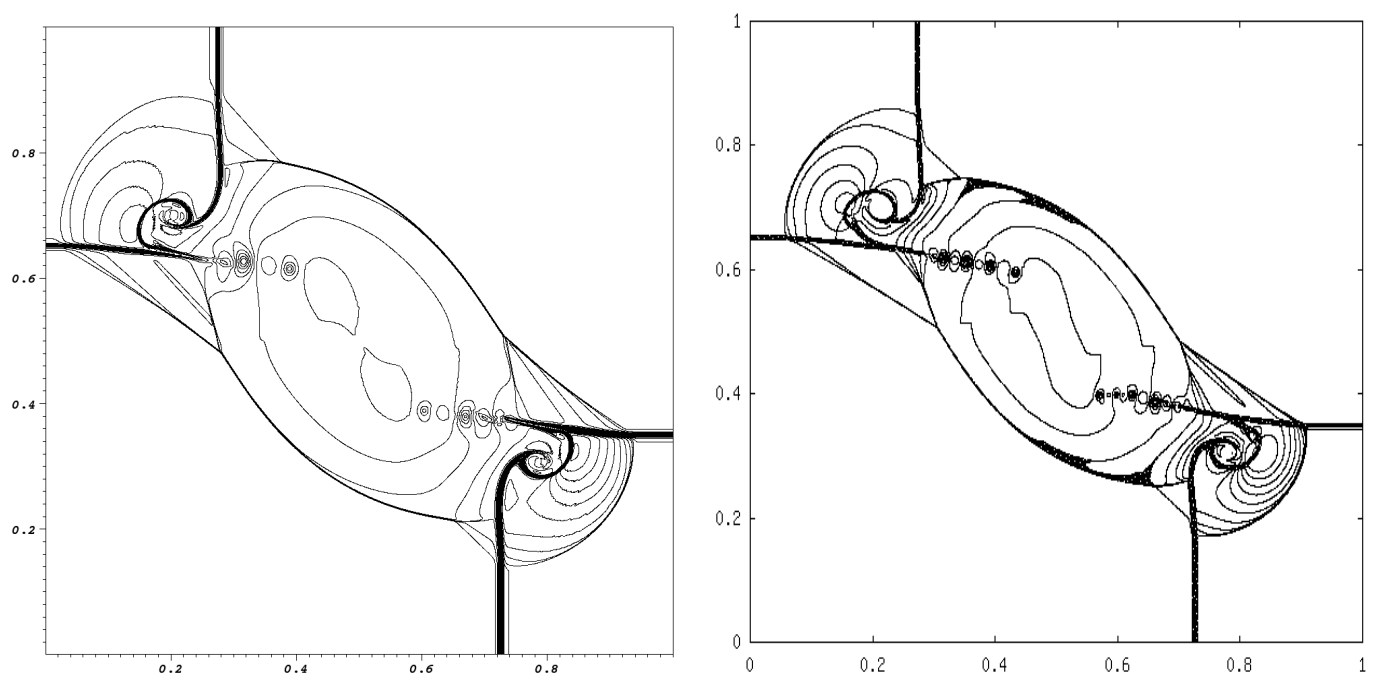

FIGURE 58. Isolines of density $\rho=1.0, \cdots, 4.0$ every 0.1 for the fine-grid reference solution at $t=0.3$, obtained with $C F L=0.45$ and $J=11$ levels, using the FV algorithms of the AMR (left) and MR schemes (right).

such reference solutions. We observe that both results differ slightly, which is likely due to the fact that the numerical fluxes used in MR and AMR methods are not exactly identical.

In Figure 59, the isolines of density for the final MR and AMR solutions at $t=0.3$ are plotted together with the adaptive grids. We observe that both solutions fit well with their respective reference solutions given in Figure 58. The grids on the right side show that both methods adapt well to the discontinuities and steep gradients of the density.

Special counters were implemented to evaluate the performance of the two codes. The CPU time compression rate is defined as the ratio between the CPU time required to compute the final solution using the adaptive method and the one required to compute the same solution using the fine-grid method. In an adaptive simulation, an average memory requirement is defined as

$$
\overline{\mathcal{C}}=\frac{1}{N_{I}} \sum_{n=1}^{N_{I}} \mathcal{C}^{n}
$$

where $N_{I}$ is the number of performed time steps, and $\mathcal{C}^{n}$ denotes the sum of cells of the entire hierarchy at $t=t^{n}$. Then, the memory compression is defined as the ratio of the average memory requirement and the number of cells $N_{C}$ of the finest uniform grid.

In a FV code, the main contribution to the CPU time is the expensive numerical flux evaluation. One crucial question is to know whether the gain in CPU time due to the reduction of expensive flux computations in adaptive simulations is larger than the additional computational overhead induced by the adaptive algorithm. To evaluate the overhead of the adaptive computations, we consider the number

$$
\Gamma_{M R}=\frac{C P U \text { Time }}{\sum_{n=1}^{N_{I}} \mathcal{L}^{n}}
$$

which denotes the average CPU time spent to evolve the solution in each cell of the computational domain, in each time step. Here $\sum_{n=1}^{N_{I}} \mathcal{L}^{n}$ denotes the sum of the leaves of the tree during the whole computation. Since 
Isolines of density

$\Delta x=1 / 1024$
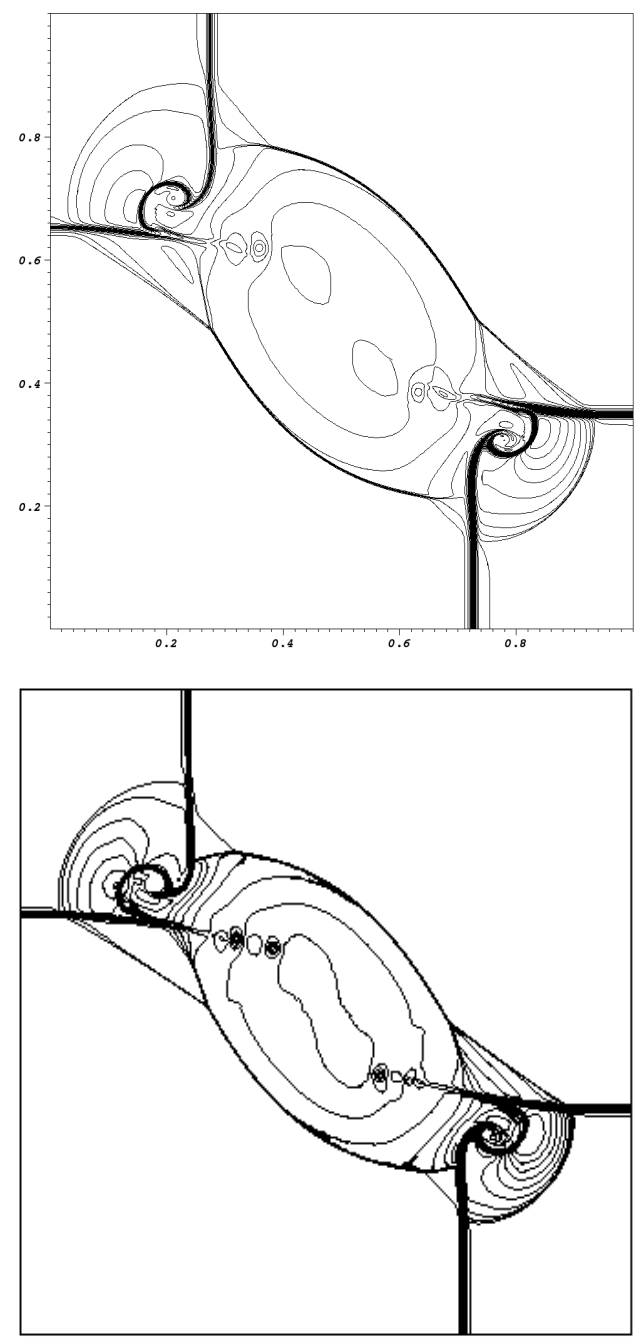

Adaptive grid

$\Delta x=1 / 1024$
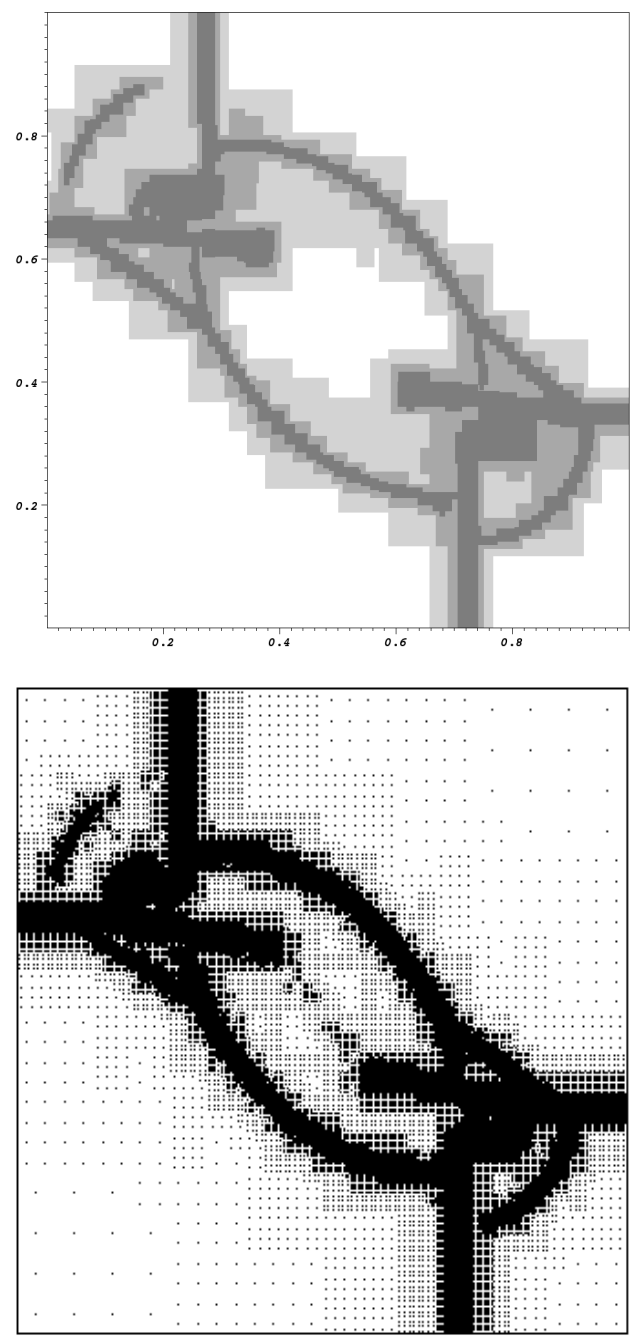

FiguRe 59. Isolines of density (left side) and corresponding adaptive mesh (right side) at $t=0.3$ with $J=10$, for the AMR scheme (top), and the MR scheme (bottom).

the time evolution of the AMR method is performed in every cell of the different adaptive grids, we define

$$
\Gamma_{A M R}=\frac{C P U \text { Time }}{\sum_{n=1}^{N_{I}} \mathcal{C}^{n}}
$$

where $\sum_{n=1}^{N_{I}} \mathcal{C}^{n}$ denotes the sum of the cells of all the adaptive grids during the whole computation.

In adaptive computations, $\Gamma_{M R}$ and $\Gamma_{A M R}$ are expected to be larger than $\Gamma_{F V}$ on the regular finest grid. Hence, the overhead per iteration and per cell of an adaptive computation is defined by 


$$
\bar{\Gamma}_{M R}=\frac{\Gamma_{M R}}{\Gamma_{F V}}-1 \text { and } \bar{\Gamma}_{A M R}=\frac{\Gamma_{A M R}}{\Gamma_{F V}}-1 .
$$

The average overhead per iteration is the overhead per iteration and per cell multiplied by the average memory compression for the AMR method, and by the average grid compression - i.e. the average number of leaves divided by the number of cells of the finest grid - for the MR method.

A summary of the MR and AMR results, obtained without and with local time stepping, is assembled in Tables 9 and 10, respectively. All the computations were run on the same double processor workstation.

With the chosen grid adaptation parameters, both adaptive methods give discrete $L_{1}$-errors of the same order. Both are comparable with the $L_{1}$-error of the $\mathrm{FV}$ scheme on the corresponding regular fine grid, as indicated in the second and third columns of Tables 9 and 10.

TABLE 9. Summary of the results for the MR and the AMR computations.

\begin{tabular}{l|c|c|ccc|cc}
\hline \hline & FV & \multicolumn{6}{|c}{ MR } \\
\hline Level & $L_{1}^{e}(\rho)$ & $L_{1}^{e}(\rho)$ & \multicolumn{2}{c}{ Compression (\%) } & \multicolumn{2}{|c}{ vverhead per iteration } \\
& {$\left[10^{-2}\right]$} & {$\left[10^{-2}\right]$} & CPU & Memory & Grid & (per leaf) & $(\%)$ \\
\hline $\mathrm{J}=8$ & 3.65 & 3.68 & 33.01 & 39.64 & 24.99 & 0.32 & 8.0 \\
$\mathrm{~J}=9$ & 2.23 & 2.26 & 20.17 & 23.40 & 14.57 & 0.38 & 5.6 \\
$\mathrm{~J}=10$ & 1.04 & 1.08 & 11.93 & 13.95 & 8.68 & 0.38 & 3.2 \\
\hline \hline
\end{tabular}

\begin{tabular}{l|c|c|ccc|cc}
\hline \hline & FV & \multicolumn{6}{|c}{ AMR } \\
\hline Level & $L_{1}^{e}(\rho)$ & $L_{1}^{e}(\rho)$ & \multicolumn{2}{c}{ Compression (\%) } & \multicolumn{2}{c}{ Overhead per iteration } \\
& {$\left[10^{-2}\right]$} & {$\left[10^{-2}\right]$} & CPU & Memory & Grid & (per node) & $(\%)$ \\
\hline $\mathrm{J}=8$ & 3.91 & 3.92 & 82.54 & 50.79 & 44.34 & 0.63 & 31.75 \\
$\mathrm{~J}=9$ & 2.34 & 2.37 & 55.62 & 28.61 & 23.02 & 0.94 & 27.00 \\
$\mathrm{~J}=10$ & 1.22 & 1.30 & 37.98 & 16.09 & 12.46 & 1.36 & 21.90 \\
\hline \hline
\end{tabular}

NOTE: Computations are performed until $t=0.3$, with $C F L=0.45$. For the MR method, the wavelet threshold is $\epsilon=0.01,0.008,0.005$ for $J=8,9$ and 10, respectively. For the AMR method, $\epsilon_{p}=\epsilon_{\rho}=0.05$ and the coarsest level is $128 \times 128$.

As expected from the previously results, we observe that the gain in both CPU time and memory compression is larger using the MR method than using the AMR one. This is particularly true for $J=8$ levels. For $J=10$ levels, the difference in terms of memory compression is slightly reduced while the difference in term of CPU time remains large. On the other hand, the speed-up due to local time stepping is larger for the AMR method than for the MR method. Table 9 shows that especially for dyadic refinement factors, and no local time stepping, the difference between the number of leaf cells and the total cell count used by the AMR method is particularly large. Nevertheless, the gain in CPU time compression is still larger using the MR/LT method than using the AMR/LT method.

Figures 60 and 61 show the time evolution of the number of used cells and the total kinetic energy for both method. They show that the kinetic energy curves match well with the reference solution, which confirms the accuracy of the method and the good grid convergence obtained on $J=10$ scales. Naturally, the MR method requires less cells than the AMR one during computation.

\subsection{Turbulence Modeling}

The intrinsic multiscale character of turbulent flows in space and time is still a major challenge in computational fluid dynamics. The numerical simulation of fully developed turbulent flows requires turbulence models to reduce the computational complexity as direct approaches are still beyond the frontiers of the available computational resources and hence they will be limited to low Reynolds number flows in the near future. In the 
TABLE 10. Summary of the results for MR/LT and AMR/LT computations

\begin{tabular}{l|c|c|ccc|cc}
\hline \hline & FV & \multicolumn{6}{|c}{ MR/LT } \\
\hline Level & $L_{1}^{e}(\rho)$ & $L_{1}^{e}(\rho)$ & \multicolumn{2}{|c}{ Compression (\%) } & \multicolumn{2}{|c}{ Overhead per iteration } \\
& {$\left[10^{-2}\right]$} & {$\left[10^{-2}\right]$} & CPU & Memory & Grid & (per leaf) & $(\%)$ \\
\hline $\mathrm{J}=8$ & 3.65 & 3.74 & 29.36 & 39.07 & 24.70 & 0.32 & 8.0 \\
$\mathrm{~J}=9$ & 2.23 & 2.38 & 17.04 & 22.94 & 14.35 & 0.39 & 5.6 \\
$\mathrm{~J}=10$ & 1.04 & 1.23 & 9.62 & 13.64 & 8.53 & 0.37 & 3.1 \\
\hline \hline
\end{tabular}

\begin{tabular}{l|c|c|ccc|cc}
\hline \hline & FV & \multicolumn{6}{|c}{ AMR/LT } \\
\hline Level & $L_{1}^{e}(\rho)$ & $L_{1}^{e}(\rho)$ & \multicolumn{2}{c}{ Compression (\%) } & \multicolumn{2}{|c}{ overhead per iteration } \\
& {$\left[10^{-2}\right]$} & {$\left[10^{-2}\right]$} & CPU & Memory & Grid & (per node) & $(\%)$ \\
\hline $\mathrm{J}=8$ & 3.91 & 3.92 & 58.73 & 38.22 & 35.00 & 0.54 & 20.5 \\
$\mathrm{~J}=9$ & 2.34 & 2.36 & 35.39 & 20.44 & 18.08 & 0.73 & 15.0 \\
$\mathrm{~J}=10$ & 1.22 & 1.27 & 22.45 & 11.40 & 10.00 & 0.97 & 11.0 \\
\hline \hline
\end{tabular}

NOTE: Computations are performed until $t=0.3$, with $C F L=0.45$. For the MR/LT method, the wavelet threshold is $\epsilon=0.01,0.008,0.005$ for $J=8,9$ and 10, respectively. For the AMR/LT method, $\epsilon_{p}=\epsilon_{\rho}=0.05$ and the coarsest level is $128 \times 128$.
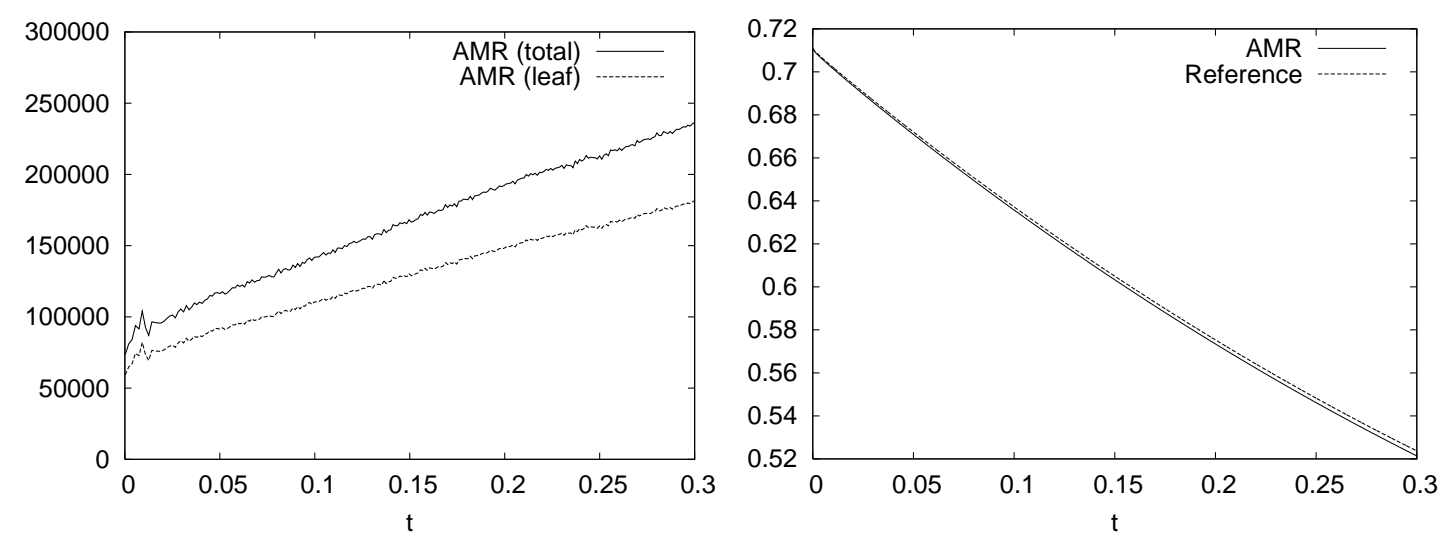

Figure 60. Time evolution of the number of used cells (left) and of the total kinetic energy (right) with $r_{l}=2,2,2$ for the AMR method using $J=10$ levels, together with the reference computation on the $2048^{2}$ mesh.

above context the compressible turbulent regime is even more challenging than the incompressible one, as in addition to the large range of involved temporal and spatial scales, shocks appear and interact non-linearly with coherent vortices. Their accurate and efficient numerical simulation becomes more complex.

Different directions for turbulence modeling have been entered upon. The large eddy simulation approach seems to be the most advanced, however reliable simulation of turbulent flow still requires the tuning of ad hoc parameters [53].

In the recent past, multiscale methods for the simulation of turbulent flows have become more and more fashionable, also for compressible flows. A review can be found in the book of Sagaut and co-workers [70]. Wavelet methods in computational fluid dynamics have been reviewed in [73].

The Coherent Vortex Simulation (CVS), which fits into the framework of multiscale methods, has been introduced by Farge, Schneider and co-workers for modeling incompressible turbulent flows $[38,39]$. The underlying 

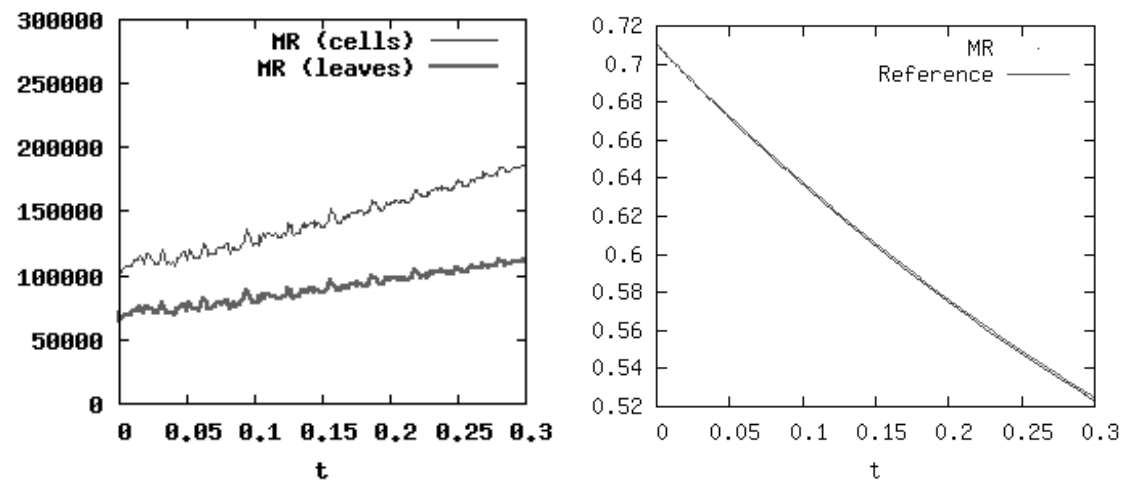

FIgURE 61. Time evolution of the number of used cells (left) and of the total kinetic energy (right) for the MR method using $J=10$ levels, together with the reference computation on the $2048^{2}$ mesh.

idea is the decomposition of the flow into coherent and incoherent contributions by means of an orthogonal wavelet filtering of the vorticity field. The coherent flow is then computed deterministically, while the influence of the incoherent background flow is statistically modeled or neglected. In Okamoto et al. [60] it has been shown that for incompressible isotropic turbulence the number of degrees of freedom $N$ required for CVS grows slower with the Reynolds number $R e$, i.e. $N \propto R e^{3.9}$, than for DNS where Kolmogorov type arguments imply $N \propto R e^{4.5}$. This motivates the development of CVS for computing fully developed turbulent flows. Adaptive space discretizations are hereby a key ingredient to be able to benefit from the efficient representation of the coherent flow to be computed in terms of memory and CPU time savings.

Adaptive multiresolution schemes have been developed in the past with the subject to speed up classical numerical discretization methods for PDEs, like finite differences or finite volumes, by locally adapting the grid to the solution which can then result in significant memory reduction. Therewith the costly evaluation of the nonlinear terms can also be reduced without degrading the precision of the solution. Three ingredients of multiresolution analysis are hereby essential: estimation of the local regularity of the solution, thresholding of weak coefficients in the multiresolution representation of the solution and fast interpolation of the solution from the locally refined grid to locally equidistant grids. A comprehensible overview on this rapidly growing topic is given in the book of Müller [58].

The aim of the present section is to present the extension of the CVS method to subsonic compressible turbulent flows using the adaptive multiresolution algorithm originally developed by Roussel and coworkers [69]. The formalism of CVS is adapted to the compressible Navier-Stokes equations which are written in primitive variables. The influence of the wavelet filtering of the conserved quantities is investigated and the choice of the filter tolerance and the normalization of the wavelets is studied.

Navier-Stokes equations for compressible fluid flows

We consider a three-dimensional compressible flow of a Newtonian fluid in the Stokes hypothesis in a domain $\Omega \subset \mathbb{R}^{3}$. Using Einstein's summation convention, the balance equations in Cartesian coordinates can be written 
in the following dimensionless form

$$
\begin{aligned}
\frac{\partial \rho}{\partial t} & =-\frac{\partial}{\partial x_{\beta}}\left(\rho u_{\beta}\right) \\
\frac{\partial}{\partial t}\left(\rho u_{\alpha}\right) & =-\frac{\partial}{\partial x_{\beta}}\left(\rho u_{\alpha} u_{\beta}+p \delta_{\alpha, \beta}-\tau_{\alpha, \beta}\right) \\
\frac{\partial}{\partial t}(\rho e) & =-\frac{\partial}{\partial x_{\beta}}\left((\rho e+p) u_{\beta}-u_{\beta} \tau_{\alpha, \beta}-\lambda \frac{\partial T}{\partial x_{\beta}}\right)
\end{aligned}
$$

In the above equations, $\alpha, \beta=1,2,3$ are the coordinate indices, $\rho, p, T$ and $e$ denote the dimensionless density, pressure, temperature and specific total energy per unit of mass, respectively; $\left(u_{1}, u_{2}, u_{3}\right)^{T}$ is the dimensionless velocity vector. The components of the dimensionless viscous strain tensor $\tau_{i, j}$ are

$$
\tau_{i, j}=\frac{\mu}{R e}\left(\frac{\partial u_{\alpha}}{\partial x_{\beta}}+\frac{\partial u_{\beta}}{\partial x_{\alpha}}-\frac{2}{3} \frac{\partial u_{k}}{\partial x_{k}} \delta_{\alpha, \beta}\right),
$$

where $\mu$ denotes the dimensionless molecular viscosity and Re the Reynolds number. The dimensionless conductivity $\lambda$ is defined by

$$
\lambda=\frac{\mu}{(\gamma-1) M a^{2} \operatorname{Re} \operatorname{Pr}},
$$

where $\gamma, M a$ and $\operatorname{Pr}$ respectively denote the specific heat ratio and the Mach and Prandtl numbers.

The system is completed by an equation of state for a calorically ideal gas

$$
p=\frac{\rho T}{\gamma M a^{2}} .
$$

and suitable initial and boundary conditions.

Assuming the temperature to be larger than $120 \mathrm{~K}$, the molecular viscosity varies with the temperature according to the dimensionless Sutherland law

$$
\mu=T^{\frac{3}{2}}\left(\frac{1+T_{s}}{T+T_{s}}\right)
$$

where $T_{s} \approx 0.404$.

Denoting by $(x, y, z)$ the three Cartesian directions, this system of equations can be written in the following compact form

$$
\frac{\partial U}{\partial t}=-\frac{\partial F}{\partial x}-\frac{\partial G}{\partial y}-\frac{\partial H}{\partial z}
$$

where $U=\left(\rho, \rho u_{1}, \rho u_{2}, \rho u_{3}, \rho e\right)^{T}$ denotes the vector of the conservative quantities, and $F, G, H$ are the flux vectors in the directions $x, y$, and $z$, respectively.

\section{Time discretization}

The time evolution is performed on the leaves of the tree only. The cell-average values in the other nodes are obtained by projection from the leaves. In order to avoid numerical diffusion, we use an explicit 2-4 Mac Cormack scheme, which is second-order accurate in time, fourth-order in space for the convective terms, and second-order in space for the diffusive terms (see, Gottlieb and Turkel [45]). The time integration is decomposed into two stages, the first stage being decentralised in one direction, the second one in the other direction. To avoid an accumulation of error in one direction, we alternate the directions at each time step. One gets the following scheme to compute the cell averages $\bar{U}^{n+1}$ at time $t^{n+1}$ from the given cell averages $\bar{U}^{n}$ at time $t^{n}$ 


$$
\begin{aligned}
\bar{U}_{j, k, m, \ell}^{*}=\bar{U}_{j, k, m, \ell}^{n}+ & \Delta t\left(\frac{\left.-7 \bar{F}_{j, k, m, \ell}^{n}+8 \bar{F}_{j, k+1, m, k}^{n}-\bar{F}_{j, k+2, m, k}^{n}\right)}{6 \Delta x_{j}}\right) \\
+ & \Delta t\left(\frac{\left.-7 \bar{G}_{j, k, m, k}^{n}+8 \bar{G}_{j, k, m+1, k}^{n}-\bar{G}_{j, k, m+2, k}^{n}\right)}{6 \Delta y_{j}}\right) \\
+ & \Delta t\left(\frac{-7 \bar{H}_{j, k, m, \ell}^{n}+8 \bar{H}_{j, k, m, \ell+1}^{n}-\bar{H}_{j, k, m, \ell+2}^{n}}{6 \Delta z_{j}}\right) \\
\bar{U}_{j, i, j, k}^{n+1}=\frac{\Delta t}{\bar{U}_{j, i, j, k}^{n}+\bar{U}_{j, i, j, k}^{*}} & \left.+\frac{-7 \bar{F}_{j, k, m, \ell}^{n}+8 \bar{F}_{j, k-1, m, \ell}^{n}-\bar{F}_{j, k-2, m, \ell}^{n}}{6 \Delta x_{j}}\right) \\
2 & \frac{\Delta t}{2}\left(\frac{-7 \bar{G}_{j, k, m, \ell}^{n}+8 \bar{G}_{j, k, m-1, \ell}^{n}-\bar{G}_{j, k, m-2, \ell}^{n}}{6 \Delta y_{j}}\right) \\
& +\frac{\Delta t}{2}\left(\frac{-7 \bar{H}_{j, k, m, \ell}^{n}+8 \bar{H}_{j, k, m, \ell-1}^{n}-\bar{H}_{j, k, m, \ell-2}^{n}}{6 \Delta z_{j}}\right)
\end{aligned}
$$

where $\Delta x_{j}=2^{-j} L_{x}, \Delta y_{j}=2^{-j} L_{y}$, and $\Delta z_{j}=2^{-l} L_{z}$ denote the space steps at a level $j$ in the three directions. Here $L_{x}, L_{y}$, and $L_{z}$, denote the lengths of the computational domain in the $x, y$, and $z$ directions, respectively.

For the computation of the diffusive terms, we do not need to use a decentralised scheme. Here the diffusive terms are approximated the same way as if we were using a second-order Runge-Kutta-Heun method in time, together with a second-order centred scheme in space.

\section{Principle of Coherent Vortex Simulation}

The CVS of incompressible turbulent flows is based on the decomposition of the vorticity $\omega=\nabla \times u$ into coherent and incoherent parts using thresholding of the wavelet coefficients. The corresponding coherent and incoherent velocity fields are then obtained by applying Biot-Savart's kernel. In the CVS method, the evolution of the coherent flow is then computed deterministically in a dynamically adapted wavelet basis and the influence of the incoherent components is statistically modeled or simply neglected $[38,39,72]$.

For compressible flows, the situation is different, since both vortical and potential components are present. Here we decided to decompose the conservative variables $U=\left(\rho, \rho u_{1}, \rho u_{2}, \rho u_{3}, \rho e\right)$ into a biorthogonal wavelet series by applying the cell-average multiresolution transform previously described. In [68], we applied the same biorthogonal wavelet decomposition to incompressible isotropic turbulence to decompose the flow into coherent and incoherent parts. We concluded that the biorthogonal decomposition yield reasonable results compared to the orthogonal wavelet decomposition typically used for CVS, which justifies the application of the former to compressible turbulence. A decomposition of the conservative variables into coherent and incoherent components is then obtained by decomposing the conservative variables into wavelet coefficients, applying a thresholding and reconstructing the coherent and incoherent contributions from the strong and weak coefficients, respectively.

First the dimensionless density and pressure are decomposed into

$$
\begin{aligned}
& \rho=\rho_{C}+\rho_{I}, \\
& p=p_{C}+p_{I} .
\end{aligned}
$$

where $\rho_{C}$ and $p_{C}$ respectively denote the coherent part of the density and pressure fields, while $\rho_{I}$ and $p_{I}$ denote the corresponding incoherent parts. 
Then the other remaining variables, i.e. the velocity components $u_{1}, u_{2}, u_{3}$, the temperature $T$ and energy $e$, are decomposed using the Favre averaging technique [40], i.e. density weighted, as done in RANS and LES of compressible flows to simplify modeling [53]. This technique avoids the introduction of unresolved terms into the equation of mass conservation and trilinear terms into the momentum equation. For a quantity $\varphi$ corresponding to one of these remaining variables, we obtain the following decomposition,

$$
\varphi=\varphi_{C}+\varphi_{I}, \text { where } \varphi_{C}=\frac{(\rho \varphi)_{C}}{(\rho)_{C}} .
$$

For sake of clarity, we use the notation $\varphi_{C}=\bar{\varphi}$. Retaining only the coherent contributions of the conservative variables we obtain the filtered compressible Navier-Stokes equations which describe the flow evolution of the coherent flow

$$
\begin{aligned}
\frac{\partial \bar{\rho}}{\partial t} & =-\frac{\partial}{\partial x_{\beta}}\left(\bar{\rho} \bar{u}_{\beta}\right) \\
\frac{\partial}{\partial t}\left(\bar{\rho} \bar{u}_{\alpha}\right) & =-\frac{\partial}{\partial x_{\beta}}\left(\bar{\rho} \bar{u}_{\alpha} \bar{u}_{\beta}+\bar{p} \delta_{\alpha, \beta}-\bar{\tau}_{\alpha, \beta}+A_{\alpha, \beta}\right) \\
\frac{\partial}{\partial t}(\bar{\rho} \bar{e}) & =-\frac{\partial}{\partial x_{\beta}}\left((\bar{\rho} \bar{e}+\bar{p}) \bar{u}_{\beta}-\bar{u}_{\alpha} \bar{\tau}_{\alpha, \beta}-\bar{\lambda} \frac{\partial \bar{T}}{\partial x_{\beta}}+B_{\beta}\right) \\
\bar{p} & =\frac{\bar{\rho} \bar{T}}{\gamma M a^{2}}
\end{aligned}
$$

where the terms

$$
\begin{aligned}
A_{i, j} & =\bar{\rho}\left(\overline{u_{\alpha} u_{\beta}}-\bar{u}_{\alpha} \bar{u}_{\beta}\right)-\overline{\tau_{\alpha, \beta}}+\bar{\tau}_{\alpha, \beta} \\
B_{\beta} & =\bar{\rho}\left(\overline{u_{\beta} e}-\bar{u}_{\beta} \bar{e}\right)+\overline{u_{\beta} p}-\bar{u}_{\beta} \bar{p}-\overline{u_{\beta} \tau_{\alpha, \beta}}+\bar{u}_{j} \bar{\tau}_{\alpha, \beta}-\lambda \overline{\frac{\partial T}{\partial x_{\beta}}}+\bar{\lambda} \frac{\partial \bar{T}}{\partial x_{\beta}}
\end{aligned}
$$

constitute the influence of the incoherent contributions. In the current paper, these terms are completely neglected. We also use the approximation $\bar{\mu}=\mu(\bar{T})$ and $\bar{\lambda}=\lambda(\bar{T})$. Note that modeling of the incoherent contributions, similar to LES approaches, has been proposed in [43], which allows for larger choices of the threshold and thus higher compression rates. A review of the different modeling strategies can be found in [73].

Choice of the thresholding rule

In the CVS of incompressible turbulence, the vorticity field is decomposed into orthogonal wavelets and filtered using a fixed threshold $\epsilon_{0}$ based on the enstrophy, i.e. on the $L^{2}$-norm of vorticity.

In most papers on adaptive multiresolution methods $[14,17,58,69]$, the conserved quantities are filtered using furthermore biorthogonal wavelets which are normalized with respect to the $L^{1}$-norm, which is well adapted to the finite volume discretization, i.e. $\left\|\widetilde{\psi}_{j,(k, m, \ell)}^{(i)}\right\|_{L^{1}}=1$. Typically, in the adaptive multiresolution method (see e.g. [69]) detail coefficients $\bar{d}_{j,(k, m, \ell)}^{(i)}=\left\langle u, \widetilde{\psi}_{j,(k, m, \ell)}^{(i)}\right\rangle$ of a conserved quantity are removed if

$$
\left|\bar{d}_{j,(k, m, \ell)}^{(i)}\right|<2^{d(j-J)} \epsilon
$$

where $d$ denotes the space dimension (here $d=3$ ), $\epsilon$ the threshold, and $J$ is the finest level present in the computations. The above choice of the threshold takes the $L^{1}$-normalization of the wavelets into account. The computations using this $L^{1}$ based thresholding rule are denoted by "Norm \#1" results.

To investigate the influence of the thresholding rule and to compare with the classical CVS scheme which uses $L^{2}$-normalization of the wavelets and filters vorticity, which corresponds to the anti-symmetric part of the velocity gradient tensor, we consider two additional thresholding rules: 
- one using the $L^{2}$-normalization of the wavelets for which we obtain the thresholding rule

$$
\left|\bar{d}_{j,(k, m, \ell)}^{(i)}\right|<2^{d(j-J) / 2} \epsilon
$$

identified later "Norm \#2" results,

- and one based on the $H^{1}$-normalization of the wavelets for which we obtain the thresholding rule

$$
\left|\bar{d}_{j,(k, m, \ell(}^{(i)}\right|<2^{(d-2)(j-J) / 2} \epsilon
$$

identified later "Norm \#3" results. The $H^{1}$ thresholding rule is motivated by the fact that here the filtering is adapted to the gradients, which mimics filtering of vorticity. However in addition to vortical regions, straindominated regions are also retained in the coherent velocity field.

To apply the threshold to the vector valued function $U$ of the dimensionless conservative quantities, we divide each component of the detail vector by the mean-value of the corresponding dimensionless quantity in the whole computational domain. Concerning the velocity vector $\left(u_{1}, u_{2}, u_{3}\right)$, in order to avoid anisotropy in the threshold of the details, we compute only one detail for the velocity

$$
\bar{d}_{j,(k, m, \ell)}^{(i)}(u)=\sqrt{\left(\bar{d}_{j,(k, m, \ell)}^{(i)}\right)^{2}\left(u_{1}\right)+\left(\bar{d}_{j,(k, m, \ell)}^{(i)}\right)^{2}\left(u_{2}\right)+\left(\bar{d}_{j,(k, m, \ell)}^{(i)}\right)^{2}\left(u_{3}\right)}
$$

and we divide this component of the detail vector by the mean-value of the velocity in the whole computational domain.

Details of all components of $U$ are removed only if all components are below the given threshold. The incoherent part $U_{I}$ corresponds hence to the conservative quantities for which, for a given index $\{j,(k, m, \ell),(i)\}$, all details are below the threshold. The coherent part $U_{C}$ corresponds to the remainder. This choice implies that only one tree data structure is necessary to store all coherent components of the conserved variables. By construction we have $U=U_{C}+U_{I}$, however the sum is not orthogonal due to the use of biorthogonal wavelets instead of orthogonal ones (see e.g. [68] for a discussion on this topic).

\section{Summary of the CVS algorithm}

In the following, we briefly summarize the CVS algorithm. For more details on the general adaptive MR algorithm, we refer to [69]. First, depending on the initial condition, an initial graded tree is created. Then, given the graded tree structure, a time evolution is made on the leaves. Finally, details are computed by multiresolution transform, in order to remesh the tree.

Denoting by $\overline{\mathbf{E}}(\Delta t)$ the discrete time evolution operator, the global algorithm can schematically be summarized by

$$
\overline{\mathbf{U}}^{n+1}=\overline{\mathbf{M}}^{-1} \cdot \mathbf{T}(\epsilon) \cdot \overline{\mathbf{M}} \cdot \overline{\mathbf{E}}(\Delta t) \cdot \overline{\mathbf{U}}^{n}
$$

where $\overline{\mathbf{M}}$ is the multiresolution transform operator, and $\mathbf{T}(\epsilon)$ is the threshold operator with tolerance $\epsilon$. The threshold operator depends on the choice of the thresholding rule.

Application to weakly compressible mixing layers

As test case for the CVS method we consider in the following a three-dimensional weakly compressible temporally developing turbulent mixing layer. For a complete description of this test-case in the LES framework, we refer the reader to $[80,81]$.

The CVS results are compared with a DNS reference computation, using a finite volume scheme on the finest regular grid. We study the impact of the different thresholding rules, the choice of the threshold, and the influence of the Reynolds number to assess the precision and efficiency of CVS. 
Flow configuration of the mixing layer

We initialize the test-case by setting two layers of a fluid stacked one upon the other one, each of them with the same velocity norm but opposed directions.

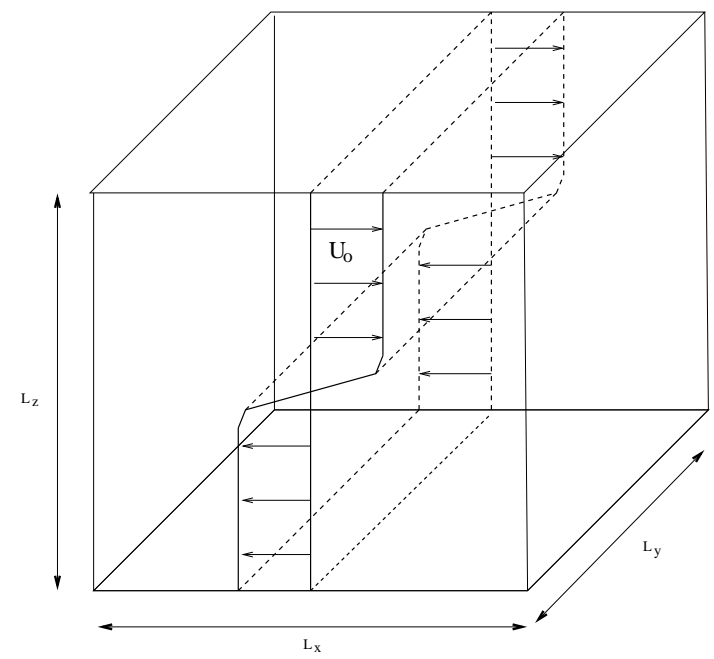

Figure 62. Flow configuration: domain and initial basic flow $\mathbf{u}_{0}$ of the three-dimensional mixing layer.

For every computation with $L=7$ scales, the computational domain is a three-dimensional cube $\Omega=$ $[-30,30]^{3}$ with side length $L_{X}=L_{Y}=L_{Z}=60$, and the final time of all computations corresponds to $t=80$. We set periodic boundary conditions for the $x$ - and $y$-direction and Neumann conditions are imposed in the $z$-direction, i.e. on the top and bottom boundaries. The Prandtl and Mach numbers are set to 0.71 and 0.3 respectively, whereas the specific heat ratio $\gamma$ equals 1.4. The CFL number is set to 0.4 and the maximal resolution is $128^{3}$, which corresponds to $L=7$ scales. Then, in section $4.5, L=8$ scales are considered, i.e. a maximal resolution of $256^{3}$.

In this test-case, the initial dimensionless velocity profile is

$$
\mathbf{u}_{0}(\mathbf{x})=\left(\begin{array}{c}
\tanh (z) \\
0 \\
0
\end{array}\right)
$$

and the initial dimensionless density and temperature profiles are constant and set to 1 .

A sinusoidal perturbation of the form

$$
u^{\prime}(\mathbf{x})=A(z)\left(\frac{1}{2} \cos \frac{2 \pi(x+y)}{L}+\frac{1}{2} \cos \frac{2 \pi(x-y)}{L}+\sum_{k=1}^{b-1} \cos \frac{2^{k+1} \pi x}{L}\right)
$$

is superimposed to the initial velocity in the streamwise direction, where

$$
A(z)=\frac{1}{b \cosh ^{2}(z)}
$$

is the amplitude of the perturbation, $L=60$ is the length of the computational domain, and $b$ denotes the maximal number of modes used for the computation, $b=3$ in this case. The $\cosh ^{2}(z)$ term is added to limit 
the perturbation to the junction of the two stacked-up fluid layers. This way, we spare unnecessary waste of CPU resources. Here, we have added only one oblique mode disturbance, so that we can more easily distinguish the structures. The same amount of intensity is given to each of the three modes.

\section{Time evolution of a mixing layer}

For the DNS reference computation, we use the finite volume scheme on the regular grid with resolution $128^{3}$. The Reynolds number based on the initial velocity and half the initial layer thickness is set to $R e=100$. Figure 63 shows the time evolution of the mixing layer. At $t \approx 19$, the Kelvin-Helmholtz instability generates four rollers in the streamwise direction (Figure 63, left side). The number of vortices at the initial stage depends on the number of modes $b$ used in the initial perturbation and on the size of the domain $\Omega$. At the beginning, the mixing layer remains approximately two-dimensional, since we have introduced only one oblique mode. Later on, the vortices begin to pair so that, at $t \approx 37$, we observe two vortex pairings (Figure 63 , center). At $t \approx 78$, these two pairings are finished and three-dimensional structures appear, generated by the oblique mode between the two remaining vortices (Figure 63, right side). For longer computational times, assuming that the domain is large enough, these two vortices would pair again, thus leading to only one vortex.

\section{Choice of the thresholding rule}

In this section, we perform a series of CVS computations for $R e=100$ using the three different thresholding rules (Norms \#1, \#2 and \#3) previously presented, and compare the results with the reference DNS computation on the finest grid, using the same numerical scheme. For each thresholding rule, we first perform several computations with different tolerances to determine its optimal value $\epsilon_{\text {opt }}$. To assess which thresholding rule yields the best results, we then compare the energy spectra, the energy and enstrophy evolutions, as well as the corresponding computational resources (CPU time and memory) that were spent. Once we have the optimal value for each norm, we compare those optimal computations and decide which norm shows better results.

Results using the $L^{1}$-normalization (Norm \#1)

In Table 11, we show the results of the CVS computations for the Norm \#1 together with the DNS reference run. We observe that the CVS computations require much less CPU time (between 26 and $40 \%$ ) and memory (between 26 and $43 \%$ ) than DNS, except for a very small value of $\epsilon=0.01$. For the latter we see that the results of CVS are almost identical with the DNS run, as energy and enstrophy are almost perfectly retained. Memory is still reduced $(<60 \%)$ but the CPU time is almost doubled with respect to DNS. The time evolution

TABLE 11. Results obtained with the Norm \#1. Percentages of CPU time (required on a Pentium IV, $2.5 \mathrm{GHz}$ ), memory compression, and errors in comparison with the DNS solution for kinetic energy $E$ and enstrophy $Z$ at $t=80$ with different $\epsilon$ and for $N=128^{3}$.

\begin{tabular}{ccccccc}
\hline Method & $\epsilon$ & CPU time & $\% C P U$ & $\%$ Mem & $\% E$ & $\% Z$ \\
\hline DNS & & 7d 6h & $100 \%$ & $100 \%$ & $100 \%$ & $100 \%$ \\
\hline MR & 0.3 & 2d $2 \mathrm{~h}$ & $28.74 \%$ & $25.96 \%$ & $99.83 \%$ & $83.19 \%$ \\
MR & 0.25 & 2d 14h & $35.63 \%$ & $29.48 \%$ & $99.92 \%$ & $83.80 \%$ \\
MR & 0.2 & 2d 15h & $36.21 \%$ & $33.44 \%$ & $99.92 \%$ & $84.85 \%$ \\
MR & 0.1 & 3d 4h & $43.68 \%$ & $42.91 \%$ & $99.89 \%$ & $94.18 \%$ \\
MR & 0.01 & 13d 20h & $190.80 \%$ & $75.93 \%$ & $99.98 \%$ & $99.99 \%$ \\
\hline
\end{tabular}

of energy shown in Figure 64 (left side) illustrates that, for all considered values of $\epsilon$, the CVS result matches well with the DNS reference solution. For the evolution of the enstrophy (Fig. 64, right side), we see however that the dynamics differs from the DNS reference, which shows that the numerical dissipation of the CVS is not sufficient.

The spectral distribution of energy, defined as the modulus square of the Fourier transform on the velocity field in the streamwise direction, which has been averaged over two remaining directions, is given in Fig. 65. 

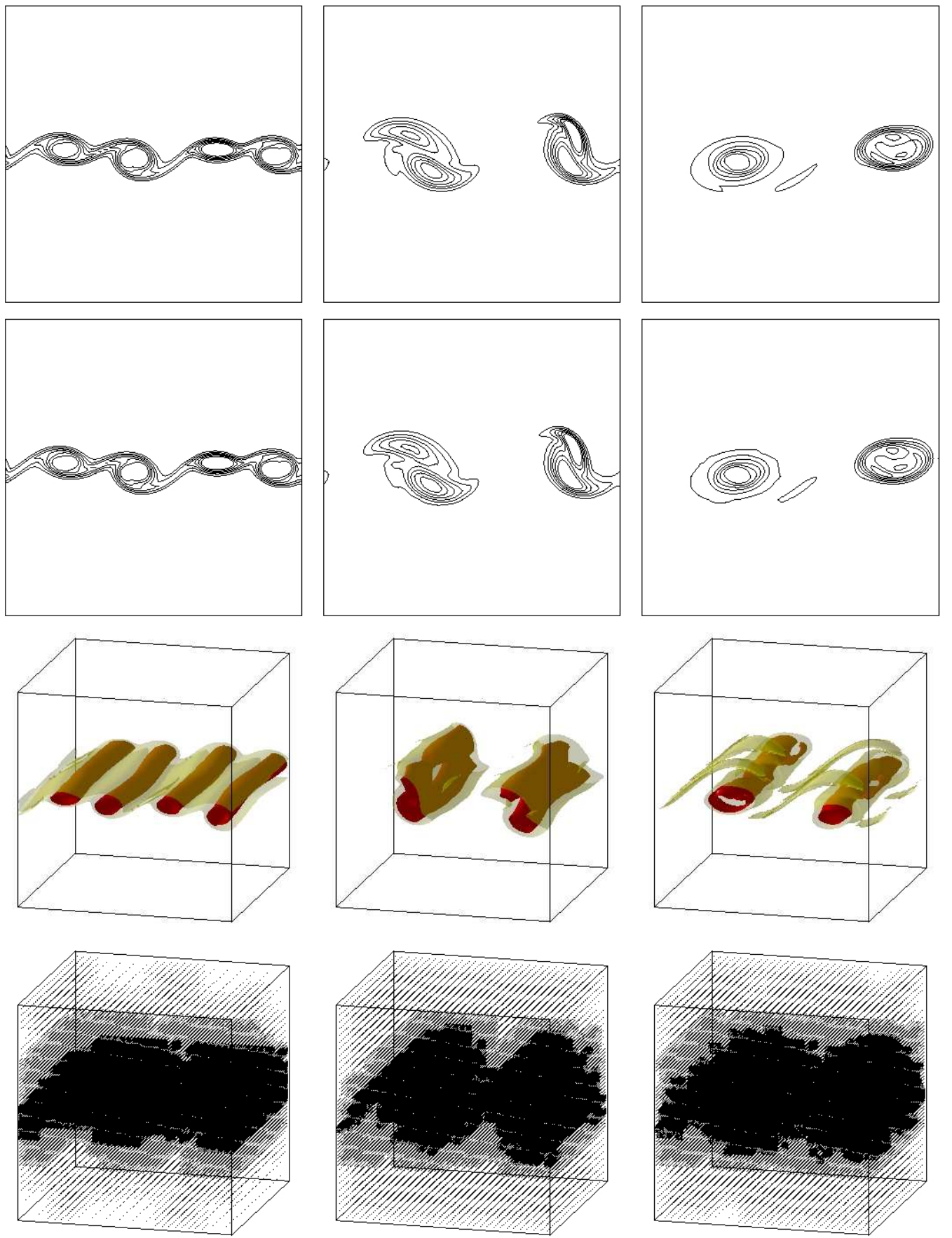

FiguRE 63. Time evolution of a weakly compressible mixing layer for $R e=100$. First row: isolines of vorticity in the plane $y=0$ for the DNS solution. Second row: same isolines for the CVS computation with $\epsilon=0.03$ and Norm \#3. Third row: Corresponding isosurfaces of vorticity $\|\omega\|=0.5$ (black) and $\|\omega\|=0.25$ (gray). Fourth row: Adaptive meshes for the CVS computation. The time instants are $\mathrm{t}=19$ (left), $\mathrm{t}=37$ (center) and $\mathrm{t}=78$ (right). 

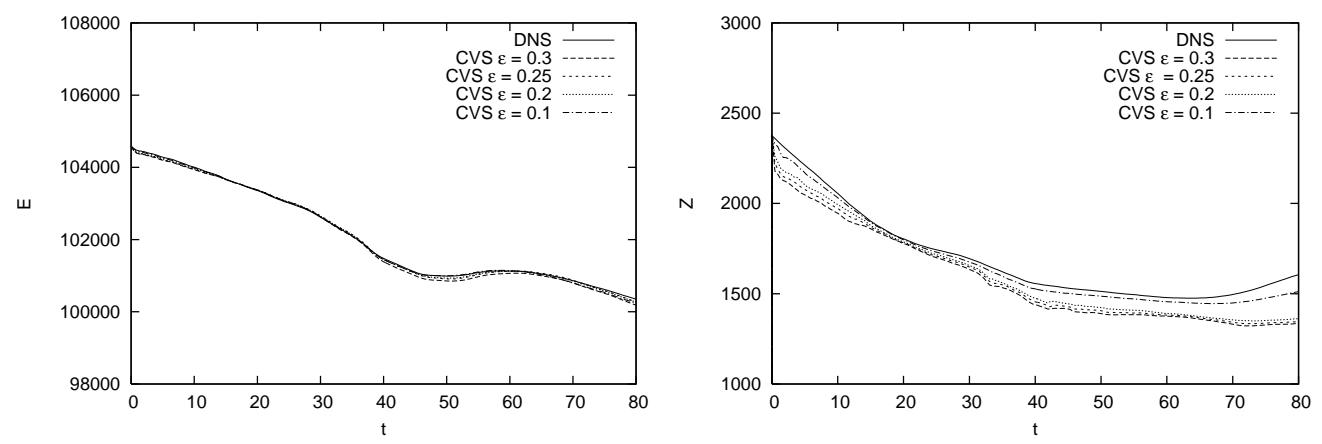

FiguRE 64. Results obtained with the Norm \#1. Kinetic energy vs time (left) and enstrophy vs time (right) for the reference DNS and the CVS with several tolerance values.

For $\epsilon=0.2$ and 0.1, the CVS results agree well with the DNS reference in the large scales, but still differ in the small scales.
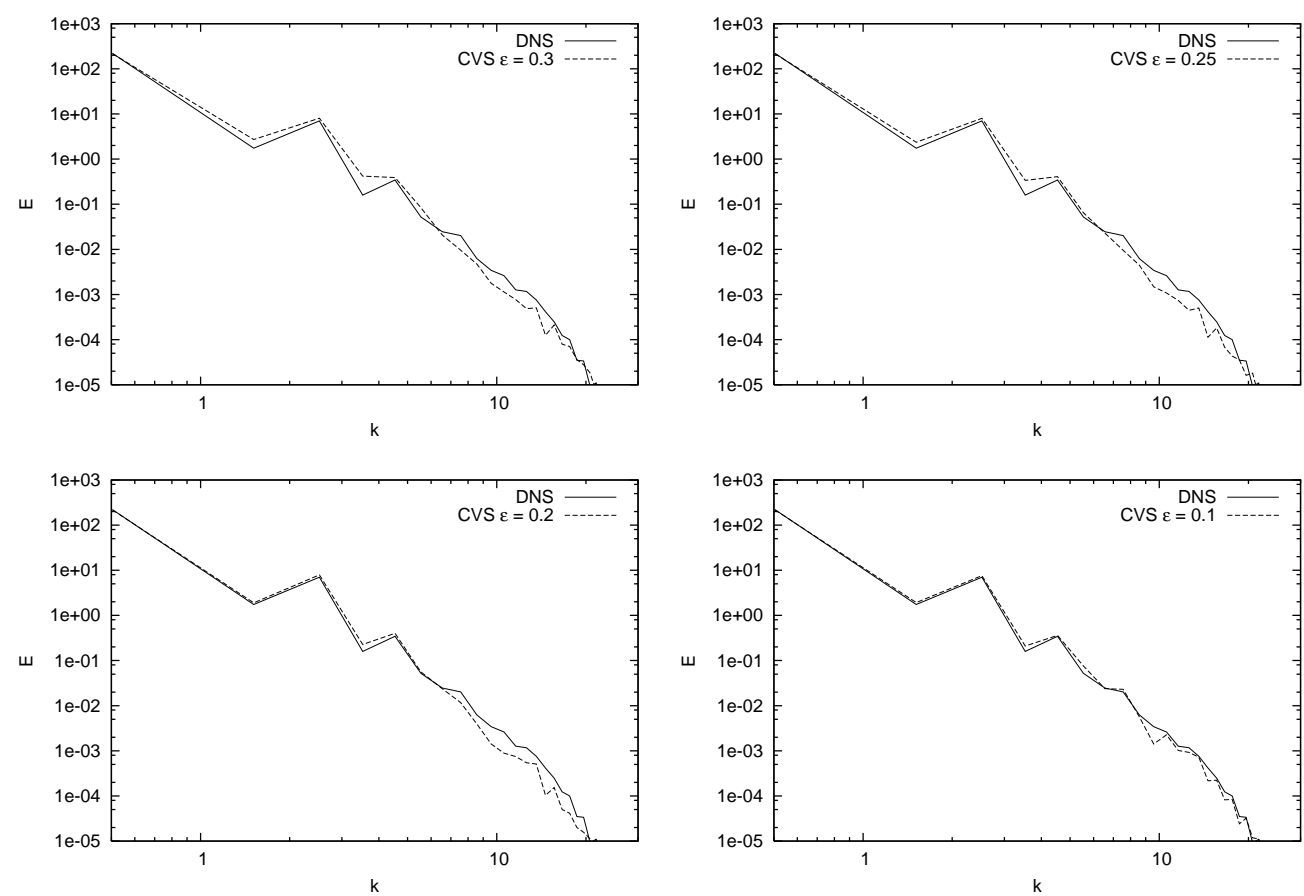

FiguRE 65. Results obtained with the Norm \#1. Energy spectra in the streamwise direction at $t=80$ for $\epsilon=0.3$ (top left), $\epsilon=0.25$ (top right), $\epsilon=0.2$ (bottom left), and $\epsilon=0.1$ (bottom right).

Results using the $L^{2}$-normalization (Norm \#2)

Table 12 shows the results of the CVS computations for the Norm \#2, together with the DNS reference computation. The CVS computations exhibit a significant reduction of CPU time and memory requirements 
with respect to the reference DNS computation, while the final kinetic energy and enstrophy are reasonably well predicted.

TABle 12. Results obtained with the Norm \#2. Percentages of CPU time (required on a Pentium IV, $2.5 \mathrm{GHz}$ ), memory compression, and errors in comparison with the DNS solution for the kinetic energy $E$ and the enstrophy $Z$ at $t=80$ with different $\epsilon$ and for $N=128^{3}$.

\begin{tabular}{ccccccc}
\hline Method & $\varepsilon$ & CPU time & $\% \mathrm{CPU}$ & $\% \mathrm{Mem}$ & $\% \mathrm{E}$ & $\% \mathrm{Z}$ \\
\hline DNS & & 7d $6 \mathrm{~h}$ & $100 \%$ & $100 \%$ & $100 \%$ & $100 \%$ \\
\hline MR & 0.5 & $7 \mathrm{~h} 57 \mathrm{~min}$ & $4.58 \%$ & $5.44 \%$ & $97.84 \%$ & $89.35 \%$ \\
MR & 0.08 & 2d $18 \mathrm{~h}$ & $37.93 \%$ & $30.55 \%$ & $99.96 \%$ & $88.17 \%$ \\
MR & 0.07 & 3d $5 \mathrm{~h}$ & $44.25 \%$ & $32.73 \%$ & $99.90 \%$ & $92.46 \%$ \\
\hline
\end{tabular}
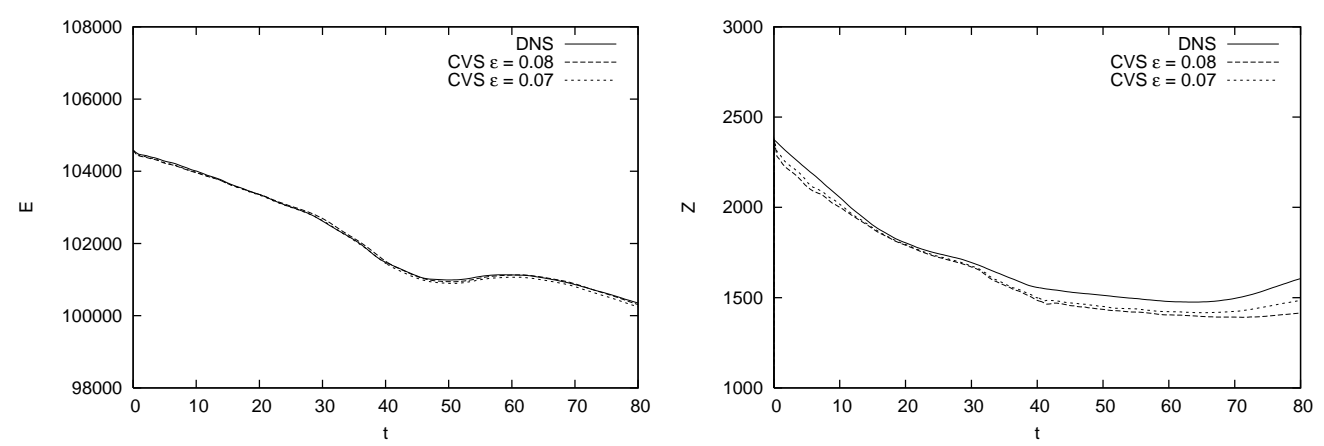

FiguRE 66. Results obtained with the Norm \#2. Kinetic energy (left), and enstrophy (right) of the DNS and several tolerance values for the CVS computations.

Figure 66 shows the time evolution of the kinetic energy and the enstrophy for the Norm \#2. The evolution of the enstrophy for the CVS computations (Fig. 66, right side) follows well the DNS reference computation until $t \approx 30$. For longer times, we observe a significant error growth. In particular for $\epsilon=0.08$, the CVS under predicts the enstrophy, the difference with respect to DNS becoming larger than $7 \%$ at $t=80$. The evolution of the kinetic energy (Fig. 66, left side) presents, as for the Norm \#1 results, a good agreement with the reference DNS computation, whatever the tolerance.

Concerning the final streamwise energy spectra (Figure 67), it is shown that the agreement between the CVS computations and the reference DNS is significantly improved when compared to the results of the Norm \#1. For the large scales, i.e. for wavenumbers $k<6$, the CVS spectra almost coincide with the DNS. However, for wavenumbers $k>6$, the differences become visible.

Still, the slight difference between CVS and DNS in the large scales $(k<6)$ involves a larger energy loss than the apparently larger energy difference in the smaller ones $(k>6)$, because of the logarithmically scaled plot. As we can observe in Table 12, the CVS with $\varepsilon=0.08$ (respectively $\varepsilon=0.07$ ) yields a relative error on energy $e_{E}$ of 0.0004 (respectively 0.0011). Norm \#2 presents better results than the Norm \#1, but the results on enstrophy are not accurate enough.

Results using the $H^{1}$-normalization (Norm \#3)

Figure 68 shows that CVS computations based on the $H^{1}$-normalization yield better results for the enstrophy and follow well the dynamics of the DNS computations. Hereby the difference between DNS and CVS decreases for decreasing threshold values and for $\epsilon=0.03$ we find the optimal agreement for the largest compression rate. These observations are confirmed in Table 13. 

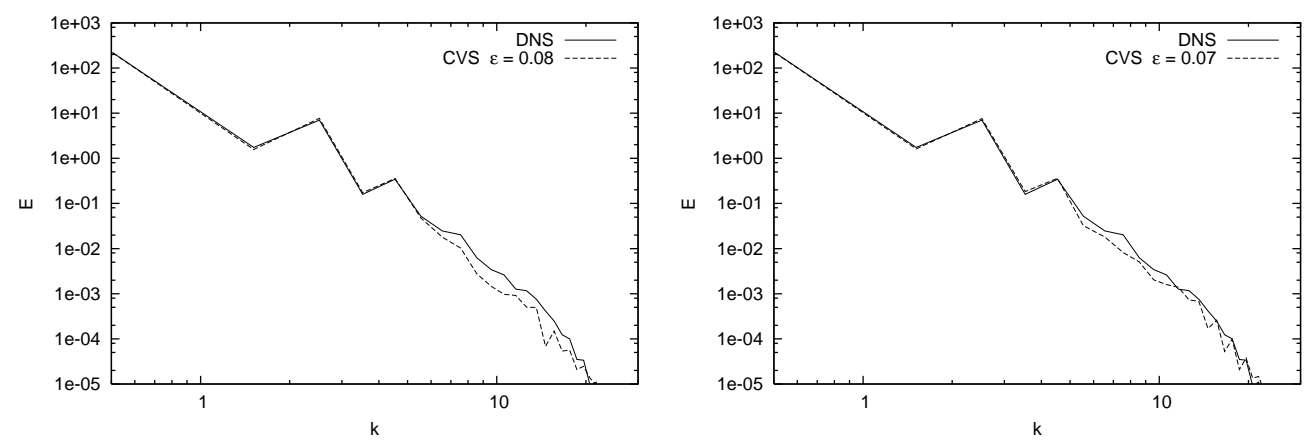

FiguRE 67. Results obtained with the Norm \#2. Energy spectra in the streamwise direction at $t=80$ for $\epsilon=0.08$ (left) and $\epsilon=0.07$ (right).
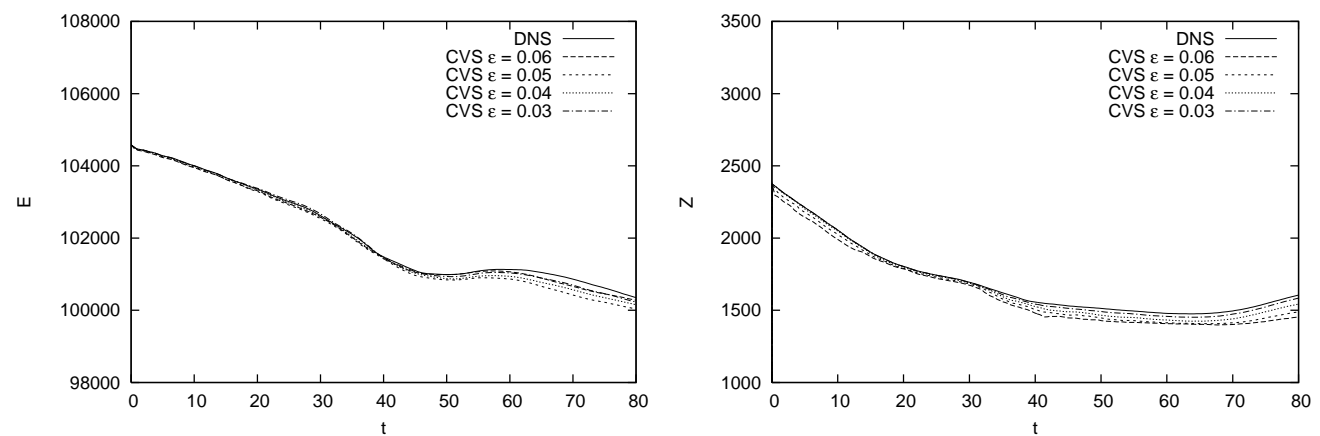

Figure 68. Results obtained with the Norm \#3. Time evolution of the kinetic energy (left) and the enstrophy (right) for the DNS and CVS computations using different tolerance values.

Figure 69 shows the final streamwise energy spectra for DNS and CVS computations with different tolerance values. Again the agreement between CVS and DNS improves for decreasing tolerances. The CVS computation with $\epsilon=0.03$ seems to be the optimal choice, since both DNS and CVS energy spectra almost coincide everywhere, whatever the wavenumber.

TABLE 13. Results obtained with the Norm \#3. Percentages of CPU time (required on a Pentium IV, $2.5 \mathrm{GHz}$ ), memory compression, and errors in comparison with the DNS solution for the kinetic energy $E$ and enstrophy $Z$ at $t=80$ with different $\epsilon, N=128^{3}$.

\begin{tabular}{ccccccc}
\hline Method & $\varepsilon$ & CPU time & $\% C P U$ & $\%$ Mem & $\% E$ & $\% Z$ \\
\hline DNS & & 7d 6h & $100 \%$ & $100 \%$ & $100 \%$ & $100 \%$ \\
\hline MR & 0.06 & 2d 0h & $27.59 \%$ & $24.23 \%$ & $99.93 \%$ & $90.60 \%$ \\
MR & 0.05 & 2d 2h & $28.74 \%$ & $27.46 \%$ & $99.69 \%$ & $92.81 \%$ \\
MR & 0.04 & 2d 10h & $29.89 \%$ & $30.80 \%$ & $99.80 \%$ & $96.12 \%$ \\
MR & $\mathbf{0 . 0 3}$ & 2d $\mathbf{8 h}$ & $\mathbf{3 2 . 1 8} \%$ & $\mathbf{3 4 . 5 4} \%$ & $\mathbf{9 9 . 8 8} \%$ & $\mathbf{9 8 . 6 6} \%$ \\
MR & 0.01 & 4d 6h & $58.62 \%$ & $48.20 \%$ & $99.95 \%$ & $99.91 \%$ \\
\hline
\end{tabular}

Table 13 shows the efficiency and accuracy of the CVS computations with the Norm \#3. We observe that only $34.54 \%$ of the memory requirements of the DNS are used to reproduce about $99.88 \%$ of the energy and 

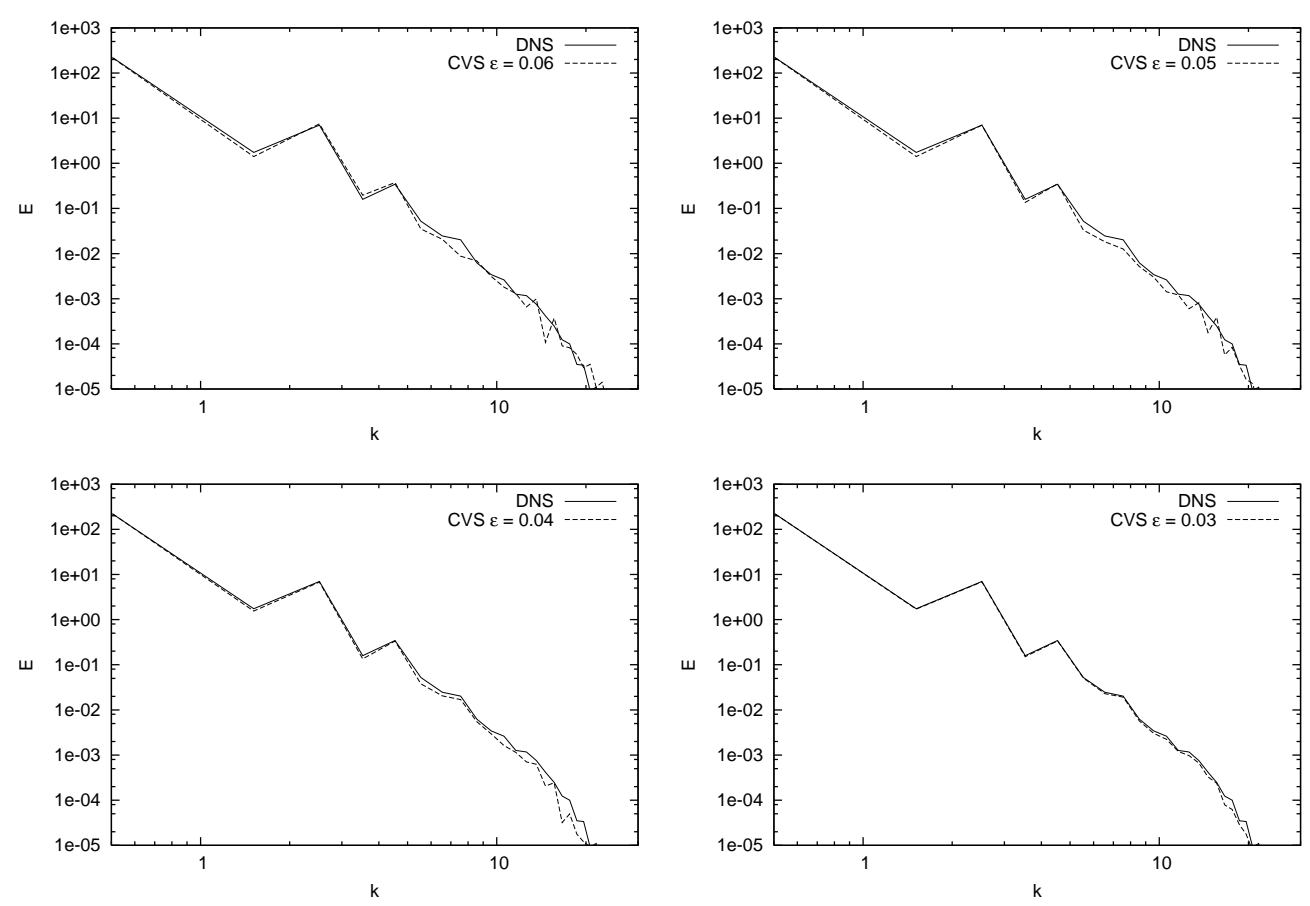

Figure 69. Results obtained with the Norm \#3. Energy spectra in the streamwise direction at $t=80$ for different tolerance values: $\varepsilon=0.06$ (top left), $\varepsilon=0.05$ (top right), $\varepsilon=0.04$ (bottom left), $\varepsilon=0.03$ (bottom right).

98.66\% of the enstrophy of the DNS computation. For this computation, less than one third of the CPU time of the DNS is required, which means that the CVS is more than three times faster than the DNS.

The vorticity fields in Fig. 63 confirm that we have a very good agreement between DNS and CVS computations with Norm \#3 and $\epsilon=0.03$. The last row of the figure presents the adaptive mesh at three different times and illustrates that the mesh dynamically adapts to the flow evolution and concentrates points in the shear layer, without any heuristic refinement criterion.

Concerning the DNS computation, it is shown that all the scales of the turbulent flow are well resolved. In fact, a reduction by a factor $10^{7}$ of energy is reached at the wavenumber $k \approx 20$. This value is much lower than the largest admissible wavenumber $k=64$, when using a resolution $128^{3}$.

\section{Influence of the incoherent part of the CVS computation (Norm \#3)}

In this part, we compute the energy spectrum of the discarded part of the CVS computation at the final time. We take the difference between the DNS and CVS computations at $t=80$. We compare this result with the DNS data where we applied the coherent vortex extraction (CVE) of the final solution using the same wavelet filtering (Figure 70).

We observe a good agreement between the filtered DNS data (Fig. 10, right) and the CVS computation (Fig. 10, left). The incoherent contributions are strongly reduced at all scales and they exhibit a $k^{-2}$ decay behaviour which corresponds to an equipartition of enstrophy, i.e. the incoherent vorticity is decorrelated in physical space. The probability distribution functions of the total, coherent and incoherent velocities, the latter obtained either from the velocity difference between the DNS and CVS computations or the velocity difference between the DNS and the CVE filtered DNS solution, are shown in Fig. 11. The results confirm the strongly reduced variance of the incoherent contributions with respect to both, the total and coherent velocities and show 

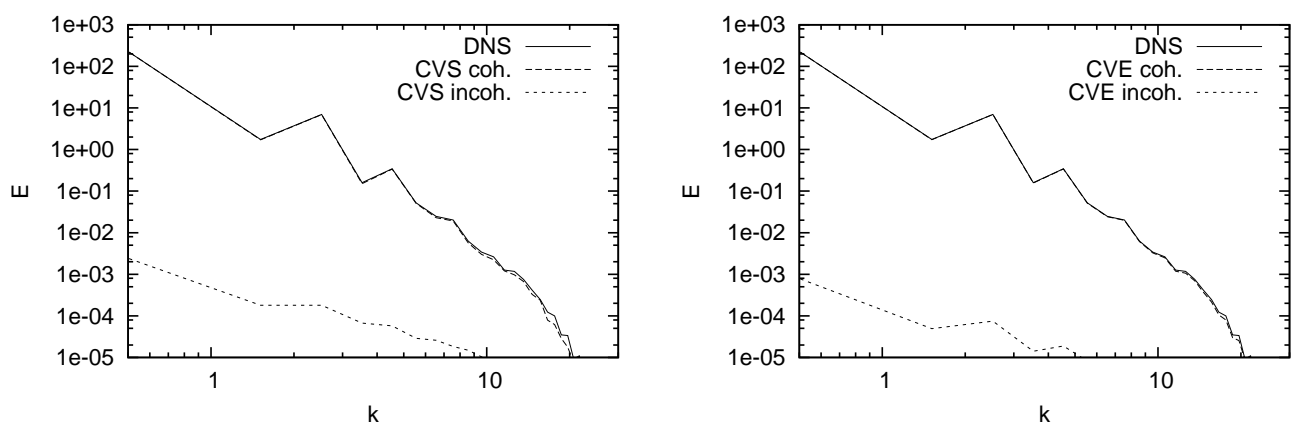

FIGURE 70. Results obtained with the Norm \#3. Energy spectra in the streamwise direction at $t=80$ for a tolerance $\varepsilon=0.03, R e=100$. Left side: incoherent part obtained from the difference between the DNS and CVS computations. Right side: incoherent part obtained from the difference between the DNS and the CVE of the final DNS solution.
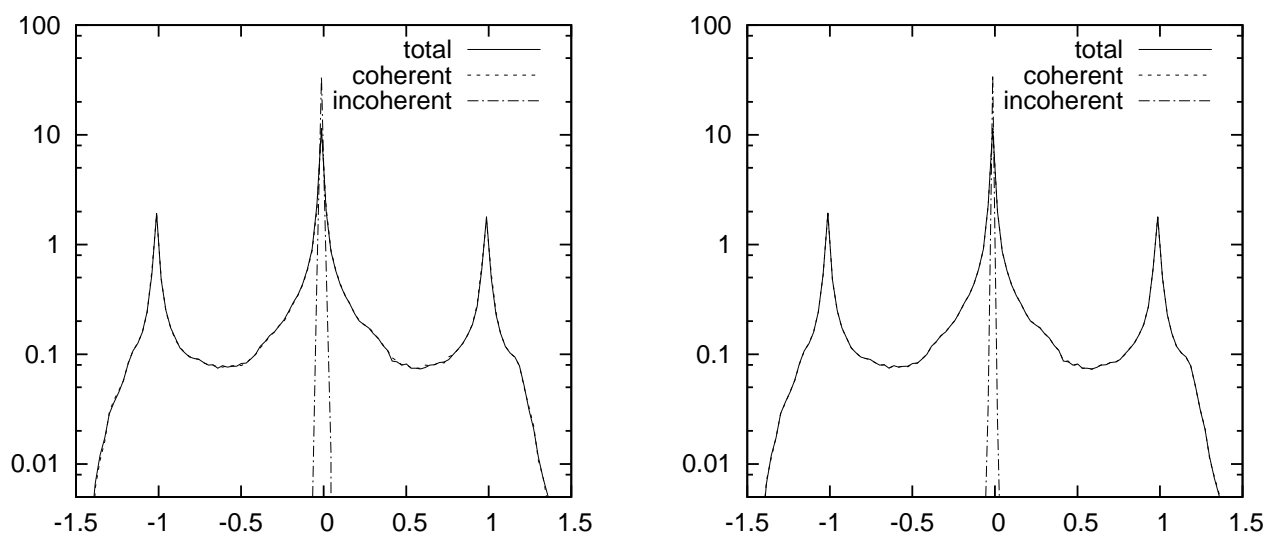

FIgURE 71. Results obtained with the Norm \#3. Probability distribution functions of the velocity at $t=80$ for a tolerance $\varepsilon=0.03, R e=100$. Left side: total, coherent and incoherent part, the latter obtained from the difference between the DNS and CVS computations. Right side: total, coherent and incoherent part, the latter obtained from the difference between the DNS and the CVE of the final DNS solution.

that the high order statistics of the CVS computation are in good agreement with the DNS. Furthermore we find that the incoherent velocity exhibits a Gaussian-like distribution. Thus neglecting incoherent contributions during the flow evolution could justify the modeling of turbulent dissipation.

\section{Influence of the Reynolds number}

In this section, we study the influence of the Reynolds number. In addition to the previous case with $R e=100$, we perform computations for $R e=50$ and 200. For every CVS computation, we use the Norm $\# 3$ and the optimal tolerance $\epsilon_{o p t}=0.03$

Results with $R e=50$

The plots in Fig. 72 show the more dissipative behaviour of the flow compared to the case with $R e=100$ (see Fig. 63). The two first rows of Fig. 72 are two-dimensional isolines of vorticity in the plane $y=0$ for both DNS and CVS computations. They confirm that the solutions agree as well as in the case $R e=100$. At the 

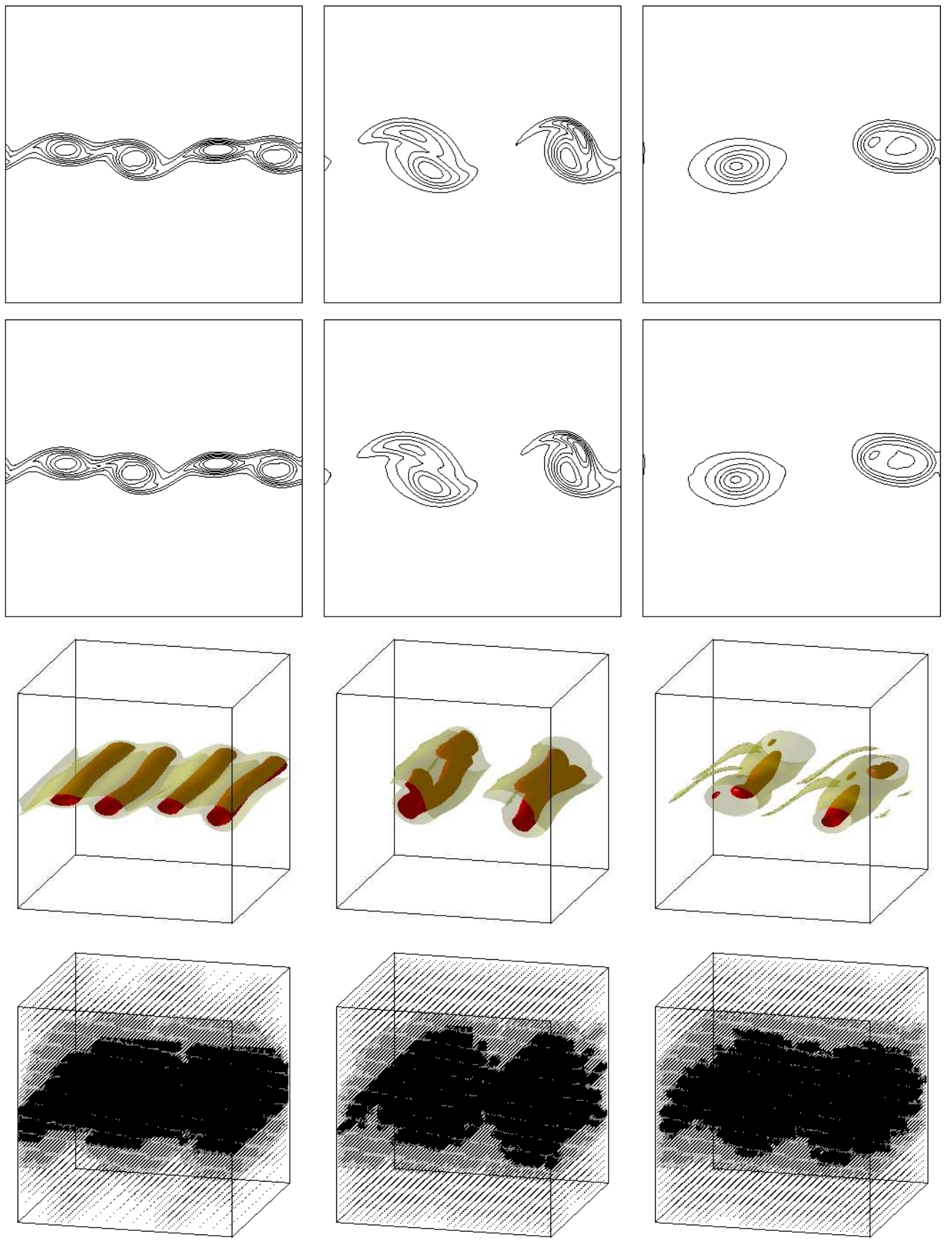

FiguRE 72. Time evolution of a weakly compressible mixing layer for $R e=50$. First row: isolines of vorticity in the plane $y=0$ for the DNS solution. Second row: same isolines for the CVS computation with $\epsilon=0.03$ and Norm \#3. Third row: Corresponding isosurfaces of vorticity of the CVS $\|\omega\|=0.5$ (black) and $\|\omega\|=0.25$ (gray). Fourth row: Adaptive mesh of the CVS computation. The time instants are $\mathrm{t}=19$ (left), $\mathrm{t}=37$ (center) and $\mathrm{t}=78$ (right). 
bottom of Fig. 72, the evolution of the adaptive mesh for the CVS computation is plotted, which confirms the efficient self-adaptive behaviour of the multiresolution algorithm.

TABLE 14. Percentages of CPU time, memory compression, and errors in comparison with the reference DNS solution for the kinetic energy $E$ and the enstrophy $Z$ at $t=80, N^{3}=128^{3}$, $R e=50$.

\begin{tabular}{cccccc}
\hline \hline Method & $C P U$ time & $\% C P U$ & $\%$ Mem & $\% E$ & $\% Z$ \\
\hline DNS & $7 \mathrm{~d}$ & $100 \%$ & $100 \%$ & $100 \%$ & $100 \%$ \\
CVS & 2d 9h & $\mathbf{3 3 . 9 3} \%$ & $\mathbf{3 3 . 7 3} \%$ & $\mathbf{9 9 . 8 2} \%$ & $\mathbf{9 8 . 6 0 \%}$ \\
\hline \hline
\end{tabular}

Table 14 gives the performance of the CVS computation in comparison with the DNS one. The percentages are very similar to the ones obtained for $R e=100$ using the optimal tolerance (see Table 13), which shows the robustness of the choice of the tolerance. The energy spectra obtained with CVS agrees well for every scale with the reference DNS (Figure 73 , left side).
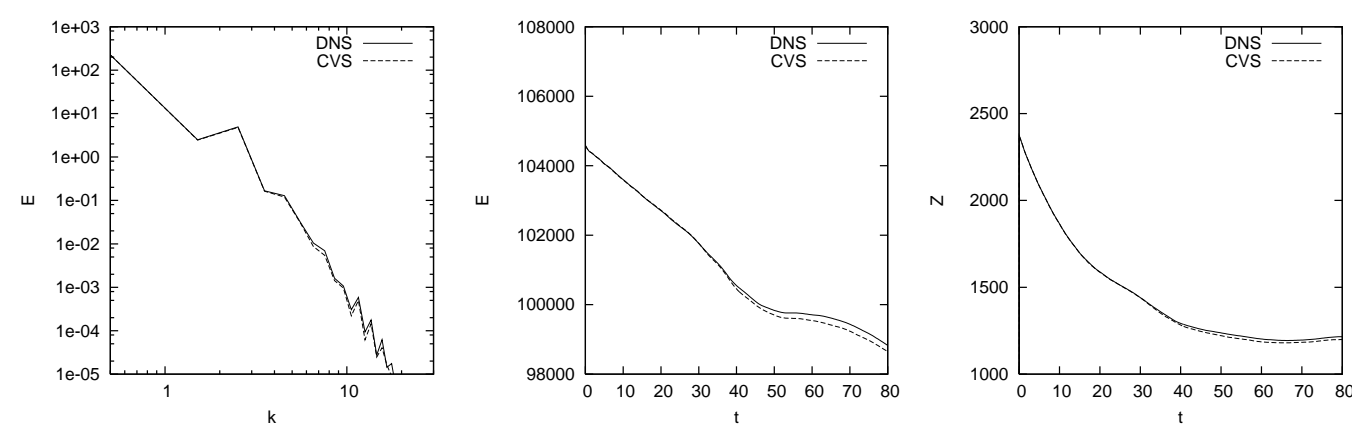

FiguRE 73. Energy spectra in the streamwise direction at $t=80$ (left), time evolution of the kinetic energy (center) and the enstrophy (right) for both DNS and CVS computations, $N^{3}=128^{3}, R e=50$.

The time evolution of the kinetic energy and the enstrophy (Figure 73, center and right side) shows likewise a good agreement between CVS and DNS.

$R e=200$

Compared with the case $R e=100$ (Fig. 63), we observe that the structures are less dissipated for $R e=200$ and that three-dimensional effects are, as expected, more intense (Fig. 74). The isolines of the two-dimensional vorticity in the plane $y=0$ (Fig. 74) look identical for CVS and DNS computations, except a slight difference for the left vortex at $t=78$.

TABlE 15. Percentages of CPU time, memory compression, and errors for $E$ and $Z$, in comparison with the DNS solution, $N^{3}=128^{3}$, Re $=200$.

\begin{tabular}{cccccc}
\hline Method & CPU time & $\%$ CPU & $\%$ Mem & $\% E$ & $\% Z$ \\
\hline DNS & 8d 14h & $100 \%$ & $100 \%$ & $100 \%$ & $100 \%$ \\
CVS & 2d $\mathbf{1 3 h}$ & $\mathbf{2 9 . 8 1} \%$ & $\mathbf{3 4 . 9 3} \%$ & $\mathbf{9 9 . 8 3 \%}$ & $\mathbf{9 8 . 2 4} \%$ \\
\hline
\end{tabular}

The results given in Table 15 for the percentages of CPU time, memory, energy and enstrophy are also very close to the ones computed with $R e=100$ (see Table 13) using the same tolerance $\epsilon=0.03$. 

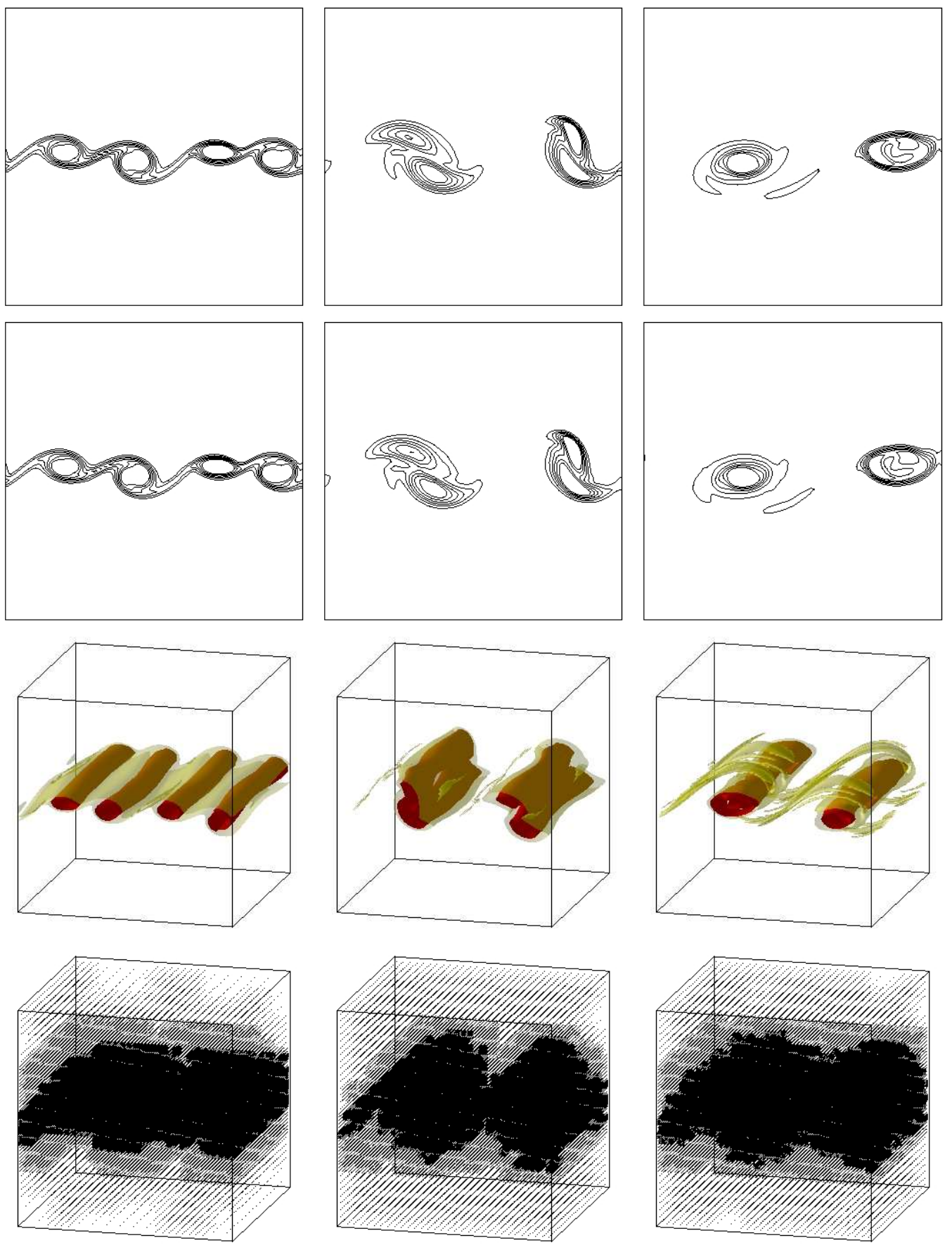

FiguRE 74. Time evolution of a weakly compressible mixing layer for $R e=200$. First row: Isolines of vorticity in the plane $y=0$ for the reference DNS solution. Second row: same isolines for the CVS computation with $\epsilon=0.03$ and norm \#3. Third row: Corresponding isosurfaces of vorticity $\|\omega\|=0.5$ (black) and $\|\omega\|=0.25$ (gray) for the CVS computation. Fourth row: Adaptive mesh for the CVS computation. The time instants are $\mathrm{t}=19$ (left), $\mathrm{t}=37$ (center) and $\mathrm{t}=78$ (right). 
The final energy spectra in the streamwise direction obtained by CVS and DNS methods are shown in Fig. 75 . They are in good accordance for almost every wavenumber. However, in the smallest scales $(k>10)$, the energy spectra loses accuracy. Despite this, the CVS computation conserves $99.82 \%$ of the energy and $98.24 \%$ of the enstrophy of the DNS, which satisfies our expectations.

The time evolution of energy and enstrophy (Fig. 75, center and right side) confirm the good agreement between the CVS and DNS computations, keeping in mind that CVS requires around one third of the CPU time and the memory required by the DNS (Table 15).
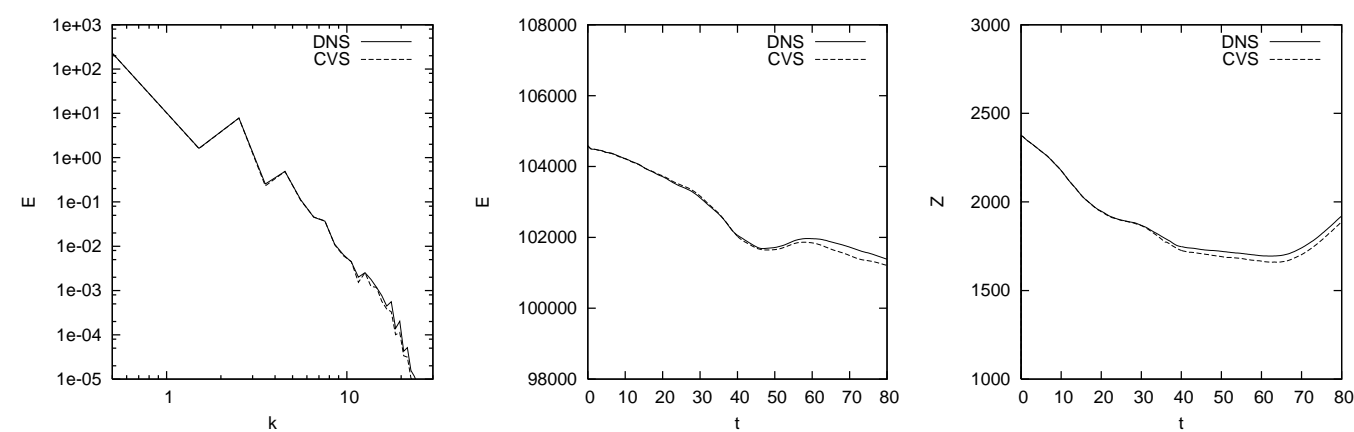

FiguRE 75. Energy spectra in the streamwise direction at $t=80$ (left). Time evolution of the kinetic energy (center) and enstrophy (right) for the CVS and DNS computations, $N^{3}=128^{3}$, $R e=200$.

\section{Comparison of energy spectra for different Reynolds numbers}

In order to assess the influence of the Reynolds number on the streamwise energy spectra, we compare computations for three Reynolds numbers (Fig. 76). In every case we find a good agreement between the CVS
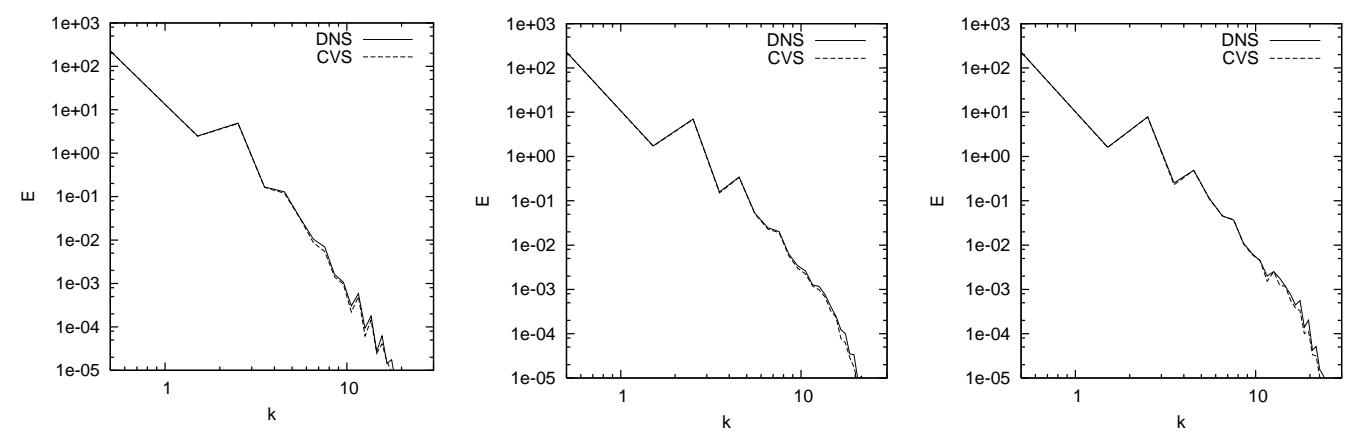

Figure 76. DNS and CVS energy spectra at $t=80$ for $R e=50$ (left), Re $=100$ (center) and $R e=200$ (right).

and DNS computations for the whole range of wavenumbers. For increasing Reynolds numbers, we also see that the range of active wavenumbers increases and that the CVS is able to well reproduce well the small scale behaviour. 
Dependence of the relative error on final energy and enstrophy with the Reynolds number

Additional computations for $R e=75, R e=125, R e=150$ and $R e=175$ have been performed, to check if the relative error of energy and enstrophy for CVS compared to DNS shows a Reynolds number dependence or remains constant. The energy and enstrophy values at $t=80$ vs the Reynolds number are plotted in Figure 77 ,
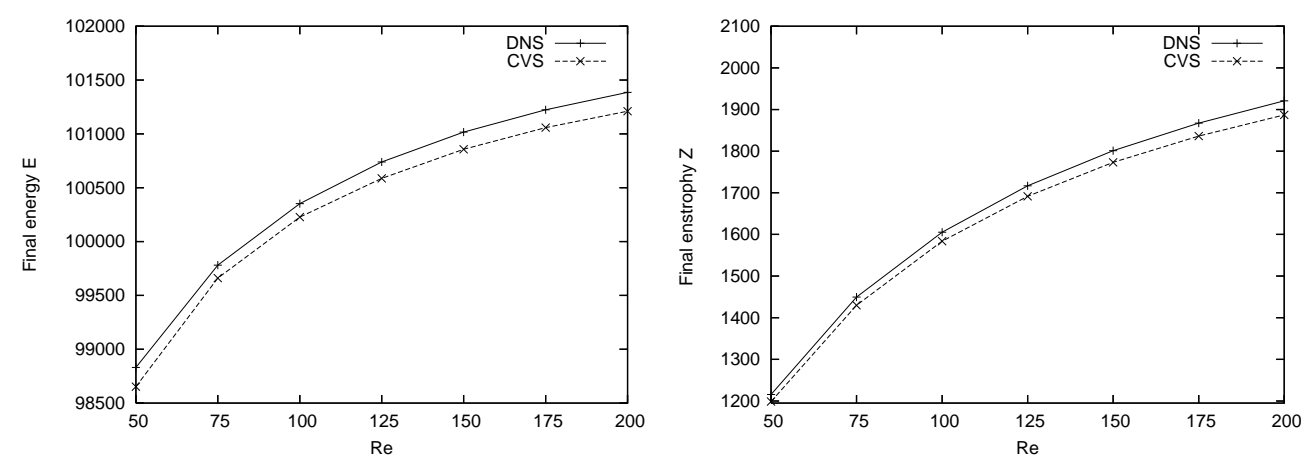

Figure 77. Final energy $E$ (left) and enstrophy $Z$ (right) vs the Reynolds number for the DNS and CVS computations.

for both DNS and CVS computations. First, we observe that both energy and enstrophy increase with Re and that the gap between CVS and DNS computations slightly increases.

However, we find that, for the kinetic energy, the relative error yields $e_{E}=1.797 \cdot 10^{-3}$ for $R e=50$, whereas, for $R e=200$, we have $e_{E}=1.722 \cdot 10^{-3}$. This means that the relative error remains almost constant. For the enstrophy, similar observations can be made, i.e. for $R e=50$ the relative error of enstrophy is $e_{Z}=1.397 \cdot 10^{-2}$ and for $R e=200$ we have $e_{Z}=1.764 \cdot 10^{-2}$. This shows that the relative error on enstrophy slightly increases, but is still of the same order of magnitude. We conclude that the relative errors on energy and enstrophy remain stable for the investigated range of Reynolds numbers.

Time evolution of a mixing layer computed at resolution $256^{3}$

Finally we present a CVS computation with norm \#3 and $\epsilon=0.03$ at resolution $N=256^{3}$. The domain is now set to $[-60,60]^{3}$, so that the smallest space step is the same as in the case $128^{3}$. As before, the Reynolds number is set to $R e=200$, and the final time corresponds to $t=80$.

Since the method has previously been validated for the same smallest space step, and since the DNS computation would require more than two months, we only perform the CVS computation here. The goal of this test-case is not to validate the method, but to show that larger CPU time and memory compressions can be reached with this method when using a larger maximal resolution. To estimate the CPU time that such a DNS computation would require, we performed the DNS computation on a few iterations only. We found that the DNS computation would approximately require 71 days of CPU time on a single PC.

The time evolution of the mixing layer is shown in Fig. 78. As before, we observe the efficient self-adaptive behaviour of the algorithm, which exhibits more points in the shear zone, and less and less points far from this zone. The performance of the CVS computation is shown in Table 16. As expected, the performance increases with the number of scales, and the CVS computation is here more than four times faster than the DNS one.

TABlE 16. Percentages of CPU time, and CPU and memory compression for the CVS computation. Results for $R e=200$ and $N^{3}=256^{3}$.

\begin{tabular}{cccc}
\hline Method & CPU time & \% CPU & \% Mem \\
\hline CVS & $\mathbf{1 6 d} \mathbf{1 7 h}$ & $\mathbf{2 3 . 4 0 ~ \%}$ & $\mathbf{1 7 . 0 6} \%$ \\
\hline
\end{tabular}



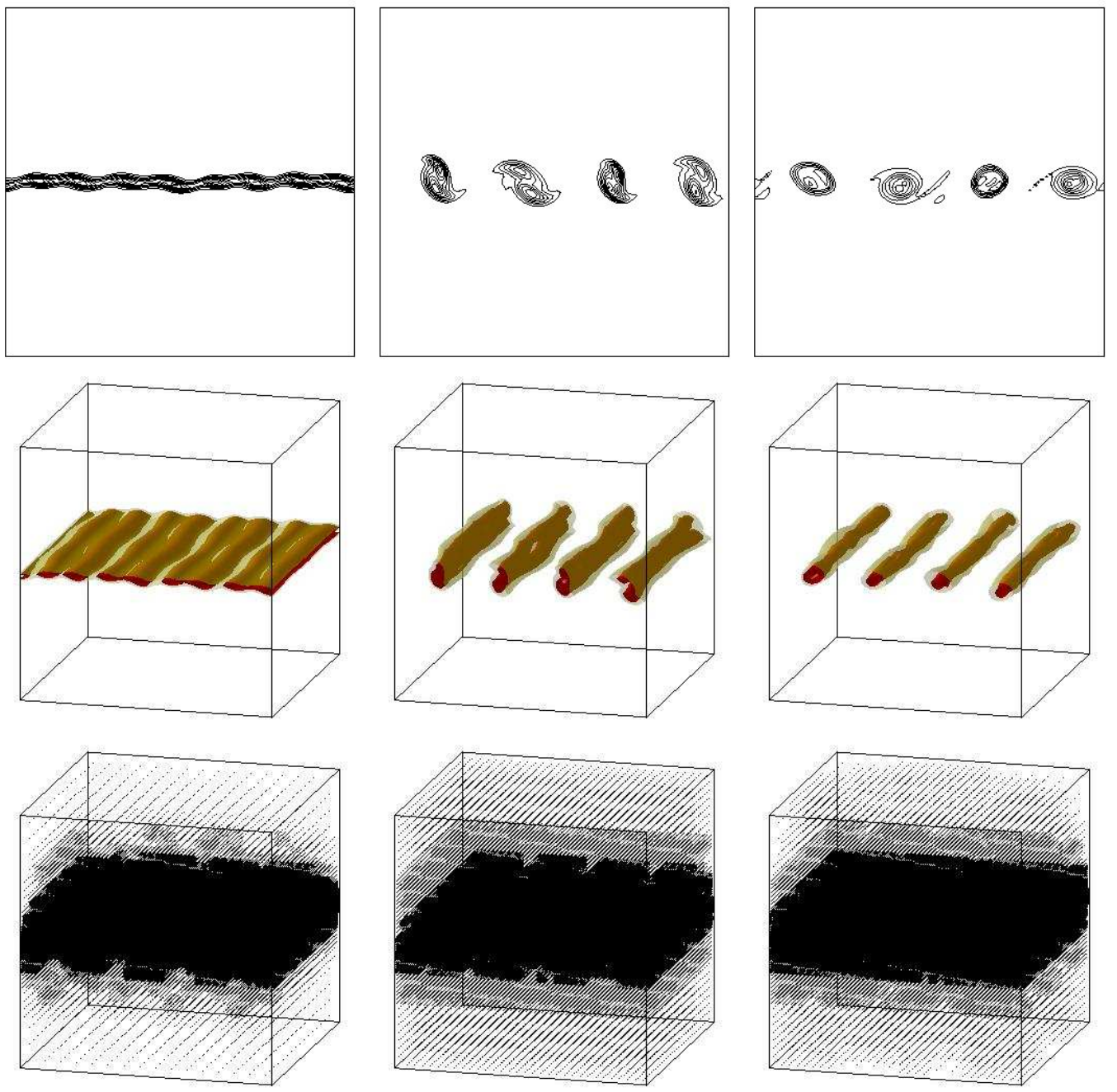

FiguRE 78. Time evolution of a weakly compressible mixing layer at resolution $N=256^{3}$. CVS computation with $\epsilon=0.03$ and norm \#3. First row: Two-dimensional isolines of vorticity in the plane $y=0,10$ isolines between 0.1 and 1. Second row: Corresponding isosurfaces of vorticity $\|\omega\|=0.5$ (black) and $\|\omega\|=0.25$ (gray). Third Row: Adaptive mesh of the CVS computation. The corresponding time instants are $\mathrm{t}=19$ (left), $\mathrm{t}=37$ (center) and $\mathrm{t}=78$ (right).

\section{Conclusions}

In these lecture notes we reviewed different adaptive multiresolution schemes for nonlinear evolutionary PDEs in Cartesian geometries, in one, two and three space dimensions. These schemes are shown to be computationally efficient and numerically accurate for a large variety of test-cases, like convection-diffusion or reaction-diffusion equations, the compressible Euler equations and the Navier-Stokes equations. The starting point of these adaptive methods are either finite volume or finite difference discretizations on regular equidistant grids, combined with explicit time integration schemes. Using discrete multiresolution analysis techniques the computational grid is reduced by deleting non significant grid points while maintaining the second order accuracy of the scheme. 

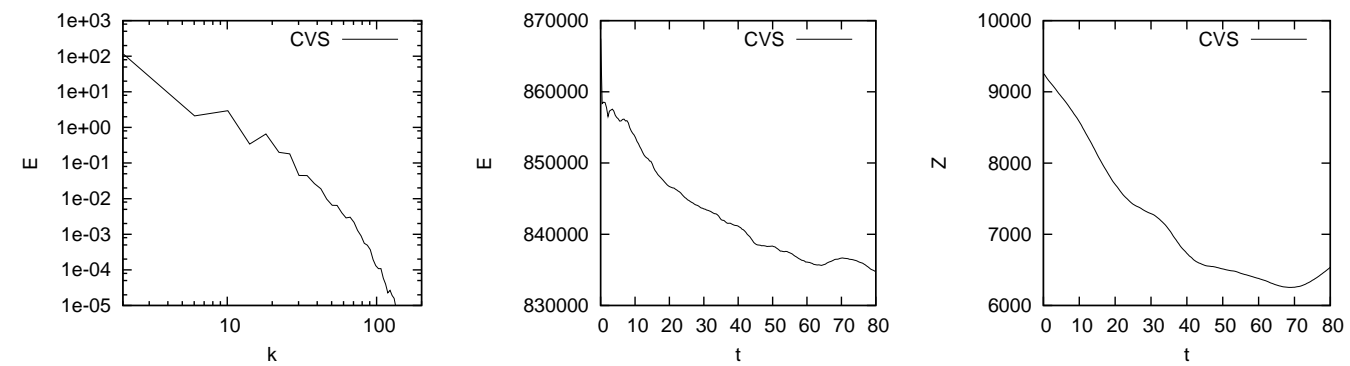

FIGURE 79. Energy spectra in the streamwise direction at $t=80$ (left). Time evolution of the kinetic energy (center) and enstrophy (right) for the CVS computations at $R e=200, N=256^{3}$.

A dynamical adaptation strategy which exploits the multiscale representation of the solution by adding neighboured coefficients in scale and space to account for translation and the creation of finer scales of the solution allows to advance the grid in time. For the evaluation of the numerical fluxes in the finite volume schemes on the locally refined grid we devise a conservative scheme without increasing significantly the number of costly flux evaluations. The presented error analysis yields a theoretical relation for the choice of a level dependent threshold for convection-diffusion equations in order to guarantee the second order accuracy, which is verified numerically. The adaptive multiresolution algorithms are implemented using graded tree data structures to represent the adaptive grid in the computer memory. A recursive procedure is used to address each element of the tree. Although this concept is slightly more complex, i.e. an $O(N \log N)$ complexity instead of $O(N)$ (where $N$ denotes the number of active grid points), this choice enables us to avoid hash-tables, which require large arrays and therefore much memory which may be prohibitive for large scale 3D computations.

We also reviewed an efficient space-adaptive multiresolution method with local time stepping to solve evolutionary PDEs in Cartesian geometry. It is again based on a finite volume discretization with explicit time integration, both of second order. In comparison to the finite volume scheme on a regular grid this new scheme allows further speed-up due to an improved time advancement using larger time steps on large scales without violating the stability condition of the explicit scheme.

However, as different scales evolve with different time steps, a synchronization of the tree data structure becomes necessary. The synchronization limits currently the time scheme to second order Runge-Kutta methods as for higher order schemes this task becomes much more difficult.

The efficiency of the different algorithms is demonstrated by comparing the performance in terms of CPU time and memory requirements with a finite volume method using the same numerical schemes on the finest regular grid with a static data structure. In general we observe that the relative performance increases with the number of required levels and tends towards a minimal value which depends on the test case. Furthermore the gain increases significantly with the spatial dimension of the problem. We have also shown that a suitable thresholding of the wavelet coefficients maintains the second order accuracy of the finite volume scheme on the regular grid. The local time stepping hence represents a moderate, but significant, speed-up with insignificant loss of accuracy.

We also presented an aspect of turbulence modelling using adaptive multiresolution techniques. Here we reviewed an extension of the Coherent Vortex Simulation approach previously developed for incompressible turbulence to weakly compressible flows. The adaptive multiresolution method was applied to solve the threedimensional compressible Navier-Stokes equations in a Cartesian geometry. It was shown that the time evolution of the coherent flow contributions can be computed efficiently using the adaptive multiresolution method. As generic test case for free shear flows, we considered weakly compressible turbulent mixing layers at low Reynolds numbers $(50<R e<200)$. Different thresholding rules based on different norms, i.e. $L^{1}, L^{2}$ and $H^{1}$ norms, have been investigated. We found that the $H^{1}$ based threshold yield the best results in terms of accuracy and efficiency. Concerning the computational efficiency, we showed that the CVS computation is approximatively 
three times faster than the DNS for a maximal resolution of $128^{3}$, and more than four times faster for a maximal resolution $256^{3}$. The CVS computation also requires less than one third of the memory that the DNS requires for a maximal resolution of $128^{3}$. In the case $256^{3}$, this factor is reduced to almost one sixth. Additionally, all the dynamically active scales of the flow are well resolved using a reduced number of degrees of freedom. The presented CVS results are of course problem dependent, i.e., on the considered flow and its dimensionless parameters. This work can be seen as a first feasibility study of CVS for weakly compressible shear flows and further investigations for higher Reynolds and Mach numbers and other flow types are necessary and will be reported in future work. Applications of the adaptive multiresolution method to supersonic flows considering the compressible Euler equations can be found in [33], which illustrates the potential of the adaptive multiresolution method for higher Mach numbers.

To further improve the compression of the CVS computations the threshold value $\epsilon$ has to be increased in order to discard more wavelet coefficients. However in this case the corresponding incoherent flow contributions cannot be simply neglected and some subgrid scale modelling is required to account for turbulent dissipation, similar to LES models. This approach called SCALES has been introduced in [43] and could be one possible direction for future work to obtain higher compression rates.

The restriction to Cartesian geometries can be surmounted using a volume penalization approach, introduced originally by Angot et al. [3] for incompressible flows and successfully applied with adaptive wavelet methods in [71]. An extension of the volume penalization to compressible flows has been published recently in [56] where, in addition to the permeability parameter, an impedance parameter is introduced to get rid of the sound waves inside the obstacle or solid wall regions. Current work is going in this direction to develop efficient CVS methods for compressible flows in complex geometries.

A hot topic and question of ongoing research for the adaptive multiresolution techniques is their parallel implementation on massively parallel machines to perform large scale 3D computations. To reach this goal, the data structure is organized as a "forest", i.e. an ensemble of trees, each one working on a different processor. Future work will focus on fully adaptive simulations of complex multi-physics phenomena with a multitude of spatial and temporal scales. 


\section{A. Multiresolution Implementation for Finite Volume Methods}

\section{A.1. Algorithms}

In the following, the principles of the FV-MR algorithm are presented. It should be emphasized that the notation may differ from the one adopted in the main text. First, depending on the initial condition, an initial graded tree is created. Then, given the graded tree structure, a time evolution is made on the leaves. Then details are computed by multiresolution transform, in order to remesh the tree. To be able to navigate inside the tree structure, we propose to use a recursive algorithm. The chosen data structure can handle $1 \mathrm{D}, 2 \mathrm{D}$ and 3D Cartesian geometries.

A flowchart of the MR algorithm for FV method is given in Fig. 80. A more detailed description is given below.

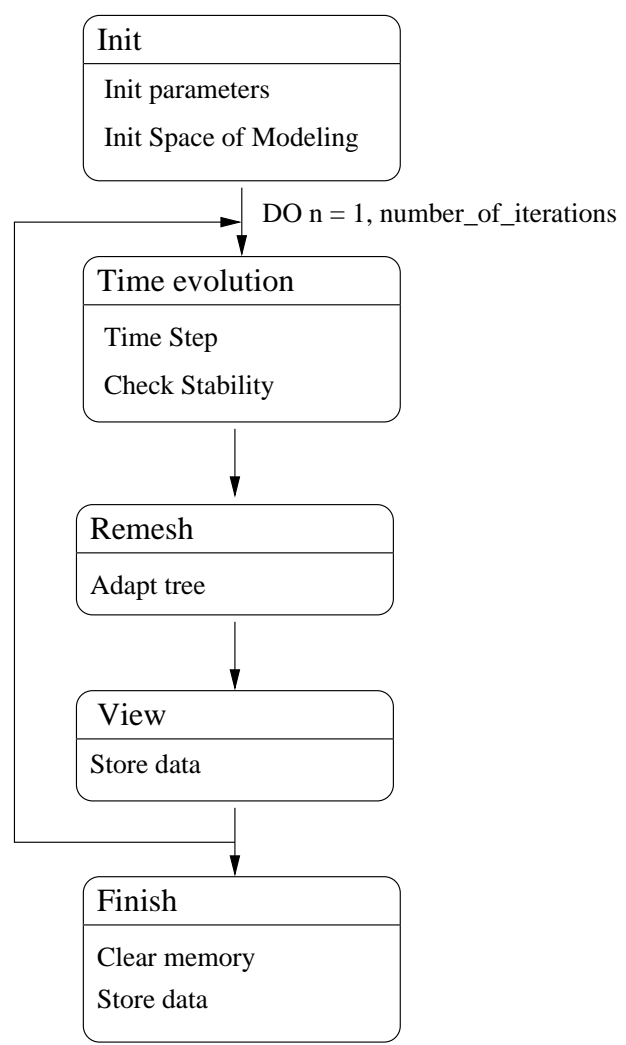

FiguRE 80. Flowchart of the main algorithm.

\section{Step 1: INITIALIZE}

- Initialize parameters e.g. number of Runge-Kutta (RK) steps, number of time steps, maximum level, domain size

- Create the initial graded tree structure

- Create first cell (root) and compute its cell-average value with the initial condition

- Split cell and compute the cell-average values in the children cells with the initial condition

- Compute details in the children cells by multiresolution transform

- IF the detail in a child cell is higher than the prescribed tolerance, THEN split this child 
- The former child becomes a parent. Repeat the same procedure until all the children cell have low details or the maximum level is reached.

: DO n=1, number_of_time_steps

Step 2: TIME EVOLUTION

- Compute Runge-Kutta steps

DO $\mathrm{m}=0$, number_of_RK_steps-1

- Compute the divergence operator for all the leaves ( except the virtual ones)

Compute $\left(\mathcal{D}_{l, i}^{n+1, m}\right)_{0 \leq l<J, i \in \mathcal{L}\left(\Lambda_{l}\right)}$

- Compute a Runge-Kutta step for all the leaves,

e.g. for number_of_RK_steps $=2$,

$m=0: \bar{u}_{l, i}^{n, 1}=\bar{u}_{l, i}^{n, 0}+\Delta t \overline{\mathcal{D}}_{l, i}^{n, 0}, 0 \leq l<J, i \in \mathcal{L}\left(\Lambda_{l}\right)$

$m=1: \bar{u}_{l, i}^{n+1,0}=\frac{1}{2}\left[\bar{u}_{l, i}^{n, 0}+\bar{u}_{l, i}^{n, 1}+\Delta t \overline{\mathcal{D}}_{l, i}^{n, 1}\right], 0 \leq l<J, i \in \mathcal{L}\left(\Lambda_{l}\right)$

END DO m

- Check Stability

IF one value is overflow THEN the computation is considered as numerically unstable

- Compute the integral values e.g. $\int_{\Omega}|u| d x$

Step 3: REMESH

- Refresh Tree

Recalculate the values in nodes and virtual leaves by projection from the leaves

- Adapt the graded tree structure

- For the whole tree from the leaves to the root $\left(0 \leq l<J, i \in \Lambda_{l}\right)$

* Compute detail in the node $d_{l, i}$ by a multiresolution transform

* IF the details in this node and in its brothers are smaller than the prescribed tolerance THEN the cell and its brothers are deletable

- For the whole tree from the leaves to the root $\left(0 \leq l<J, i \in \Lambda_{l}\right)$

* IF this node and all its children nodes are deletable

AND the children nodes are simple leaves (i.e. they are leaves without virtual children)

THEN delete children

(This means that we keep one more level)

* IF this node has no children

AND it is not deletable

AND it is not at the maximum level

THEN create the children for this node

Step 4: OUTPUT

(This means that we add one more level for each undeletable leaf)

Write the mesh and the cell-average values into a file.

: END DO $\mathrm{n}$

Step 5: FINISH

Deallocate tables

Thus, the algorithm can schematically be summarized by

$$
\bar{u}^{n+1}=\overline{\mathbf{M}}^{-1} \cdot \mathbf{T}(\epsilon) \cdot \overline{\mathbf{M}} \cdot \overline{\mathbf{E}}(\Delta t) \cdot \bar{u}^{n}
$$

where $\overline{\mathbf{M}}$ is the multiresolution transform operator, $\overline{\mathbf{M}}^{-1}$ its inverse operator, $\mathbf{T}(\epsilon)$ the thresholding operator with the prescribed tolerance $\epsilon$, and $\overline{\mathbf{E}}$ the discrete time evolution operator, as defined in (19). 

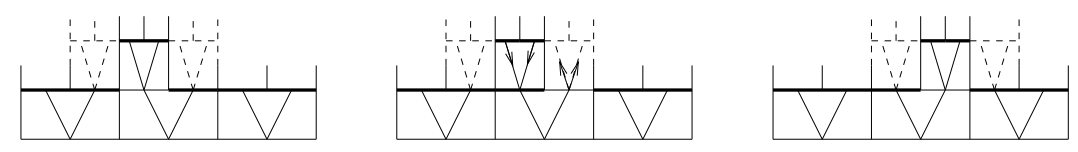

FiguRE 81 . Time evolution of the tree structure at time step $t^{n}$ (left), at time step $t^{n+1}$ before remeshing (middle), and at time step $t^{n+1}$. after remeshing (right). Deletable cells are represented by a bold line, undeletable cells by a thin line, and virtual leaves by a dotted line.

\section{A.2. Data structure}

In the implementation of the algorithm, we use a dynamic graded tree structure to represent data in the computer memory. The adaptive grid corresponds to a set of nested dyadic grids generated by refining recursively a given cell depending on the local regularity of the solution.

In the program, the tree is represented by a set of nodes with links between them. The main element of the tree structure is the node, which consists of a set of geometric and physical quantities, and pointers to the children, which are in the next finer subgrid. The root is the first cell of the tree structure.

Each node can be addressed by its level number $l$ and its coordinate in this level $i(i, j$ in $2 \mathrm{D}$, and $i, j, k$ in 3D). The procedure GetNode achieves this goal: starting from the root element, it finds the path to the cell, level by level, by opting for the child which is an ancestor of the target cell.

Thus, any node can be found by a maximum of $J$ steps, $J$ being the maximal level number. Denoting by $N_{\max }$ the maximal number of cells, we have $N_{\max }=2^{d J}$, i.e. $J=\frac{1}{d} \log _{2} N_{\max }, d$ being the dimension. Therefore, in the worse case, the global algorithm is of $O(N \log N)$ complexity. Nevertheless, for a highly compressed solution, the $J$ steps are required only in small regions and the number of cells is usually much smaller than $N_{\max }$.

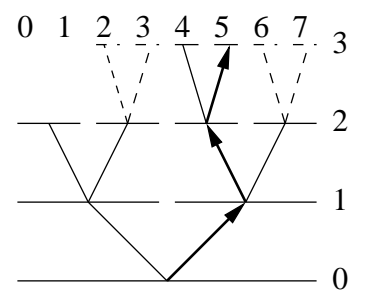

Figure 82. Path used by GetNode to reach the node $l=3, i=5$.

Another possibility is to avoid a GetNode procedure and to use a hash-table. In this case, the algorithm is at least of $O(N)$ complexity. However, this table can be large and requires much memory, especially for large scale $3 \mathrm{D}$ computations. That is why we decided here to opt for a solution which optimizes the memory requirements, with a slightly more complex algorithm.

\section{A.3. Local Scale-Dependent Time Stepping}

We propose a MR/LTS algorithm that uses the multiresolution framework described in the previous section. Assuming that $\Delta t$ is the time step for cells in the finest scale level $J$, the main principle is that the cells at lower levels $J-l$ are evolved with time step $2^{l} \Delta t$. Consequently, if $\bar{U}_{L T S}^{n}$ represents the numerical solution at $t^{n}=n \Delta t$ on the adaptive grid $\Gamma^{n}=\Gamma_{J}^{n} \subset \Omega_{J}$, then one complete time cycle of the local time stepping evolution operator evolves the solution from $t^{n}$ to $t^{n+2^{J}}$. This adaptive time strategy combined with the multiresolution scheme gives the fully adaptive time cycle 


$$
\bar{U}_{L T S}^{n+2^{J}}=\left(\mathbf{T}(\epsilon) \mathbf{E}_{L T S} \mathbf{R}\right) \bar{U}_{L T S}^{n}
$$

where $\mathbf{E}_{L T S}$ denotes the evolution operator using the adaptive multiresolution $\mathrm{FV}$ discretization combined with a scale-dependent time stepping. Recall that $\mathbf{T}(\epsilon)$ corresponds to the coarsening (thresholding), and $\mathbf{R}$ to the refinement operations.

In Figure 83, the basic ideas of the local scale-dependent RK2 time stepping are presented for one time cycle and two scale levels. First, we compute one step of RK2 with the local time step. In case the cell level is not on the finest level, we store its values and we use it to interpolate the cell-average value for the previous half local time step, that was not computed (see Figure 83, top-left). Then, with these interpolation values, we perform the RK2 update on the values for the neighbours on the finest level if they exist (see Figure 83, top-right). Following it, we return the cell-average value that we have already stored (see Figure 83, bottom-left). Finally, we advance in time the finest levels and we perform the RK2 usual update (see Figure 83, bottom-right).

We recall that:

- We are working with a spatially graded tree data structure, so we only have one level difference between two neighbour cells.

- We only permit prediction operations on the tree data structure when one complete time cycle is finished. This means that coarsening of the mesh is forbidden during the LTS time cycle, but refinement is possible.

For the whole adaptive tree, one can summarize this cycle of time evolution as follows:

I. Input leaves and time step (Algorithm A.1).

II. Compute LTS time evolution cycle (Algorithm A.2).

III. Adapt tree, update the tree values, combining or splitting them (Algorithm A.5).

More details are presented in Algorithms A.1 to A.5, which are given in the following. Here $\mathcal{L}(\Lambda)$ are the leaves of the tree $\Lambda$ and $\mathcal{V}(\Lambda)$ are the virtual leaves of the tree. The symbol $\sim$ denotes the temporary values.

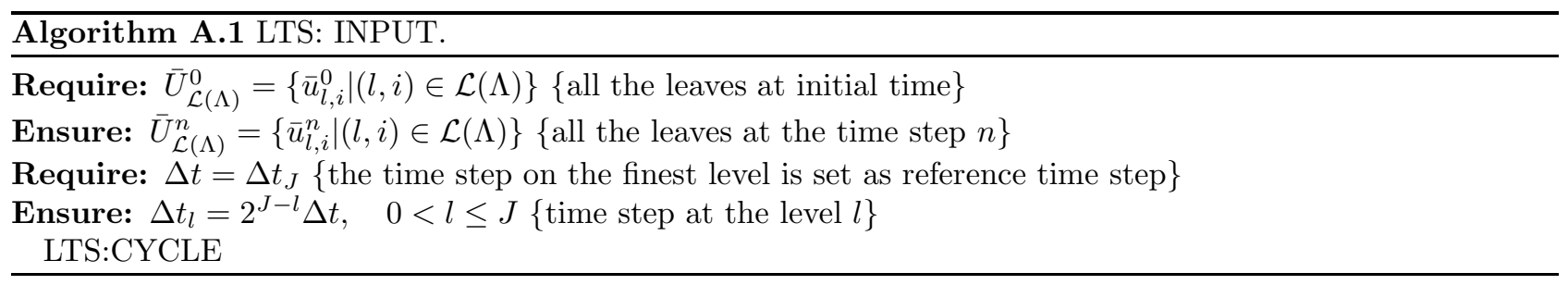

\section{REFERENCES}

[1] R. Abgrall and A. Harten. Multiresolution representation in unstructured meshes. SIAM J. Numer. Anal., 35(6):2128-2146, 1998.

[2] J. Alam, N.-K.-R. Kevlahan, and O. V. Vasilyev. Simultaneous space-time adaptive wavelet solution of nonlinear partial differential equations. J. Comput. Phys., 214:829-857, 2006.

[3] P. Angot, C.-H. Bruneau, and P. Fabrie. A penalisation method to take into account obstacles in viscous flow. Numer. Math., $81: 497-520,1999$.

[4] E. Bacry, S. Mallat, and G. Papanicolaou. A wavelet based space-time adaptive numerical method for partial equations. $M^{2} A N$ Math. Model. Numer. Anal., 26(7):793-834, 1992.

[5] R. Becker and R. Rannacher. An optimal control approach to error control and mesh adaptation. Acta Numer., 10:1-102, 2001.

[6] J. Bell, M. J. Berger, J. Saltzman, and M. Welcome. Three-dimensional adaptive mesh refinement for hyperbolic conservation laws. SIAM J. Sci. Comput., 15:127-138, 1994.

[7] M. J. Berger and R.J. LeVeque. Adaptive mesh refinement using wave-propagation algorithms for hyperbolic systems. SIAM J. Numer. Anal., 35:2298, 1999.

[8] M. J. Berger and J. Oliger. Adaptive mesh refinement for hyperbolic partial differential equations. J. Comput. Phys., 53:484512, 1984. 


\section{RK2}

\section{1st. time step}
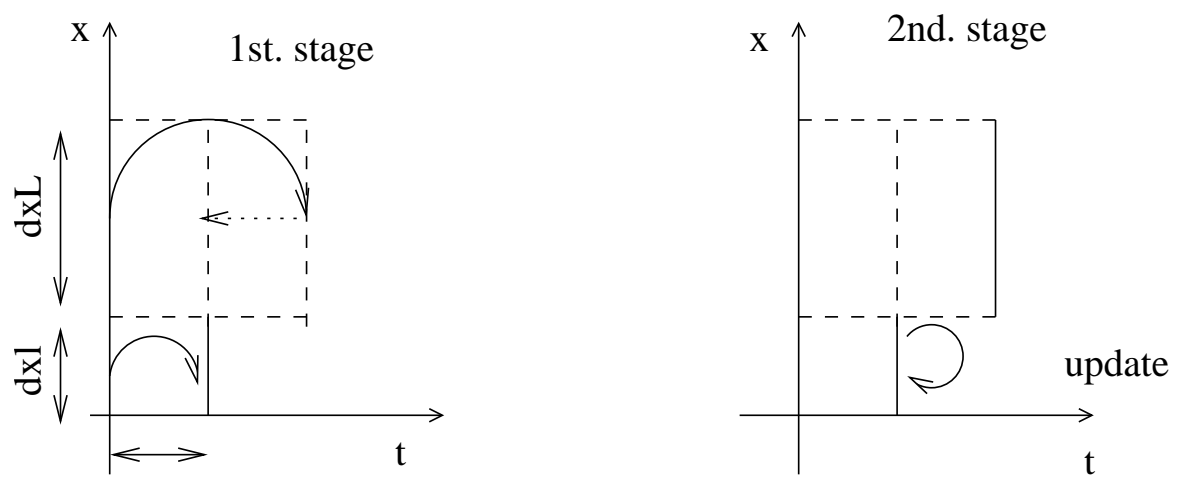

$\mathrm{dt}$

$\mathrm{dtL}$

2nd. time step
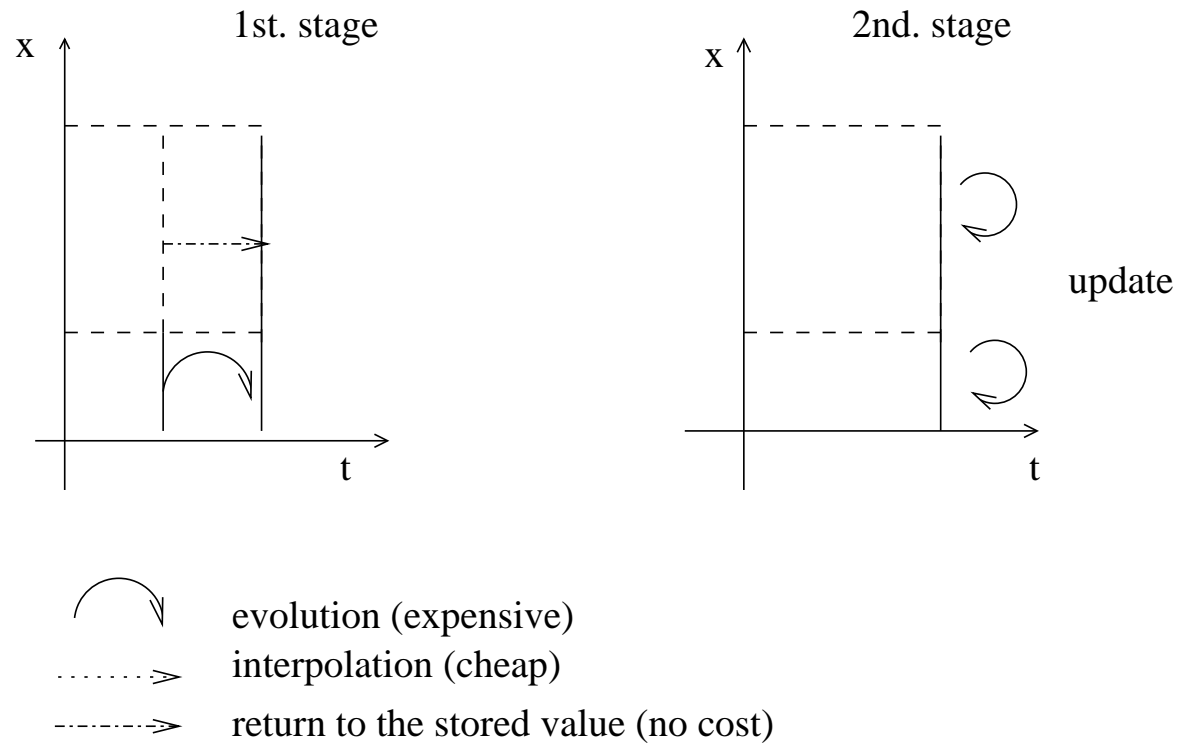

Figure 83. Scheme of local scale-dependent time stepping.

[9] B. L. Bihari. Multiresolution schemes for conservation laws with viscosity. J. Comput. Phys., 123:207-225, 1996.

[10] B. L. Bihari and A. Harten. Multiresolution schemes for the numerical solution of 2-D conservation laws I. 18(2):315-354, 1997.

[11] J. D. Calle, P. R. B. Devloo, and S. M. Gomes. Wavelets and adaptive grids for the discontinuous Galerkin method. Numer. Algor., 39:143-158, 2005.

[12] J. E. Castilho and S. M. Gomes. A multilevel wavelet scheme for evolution equations with time adaptivity. Technical report, Unicamp, Campinas, April 2002. 

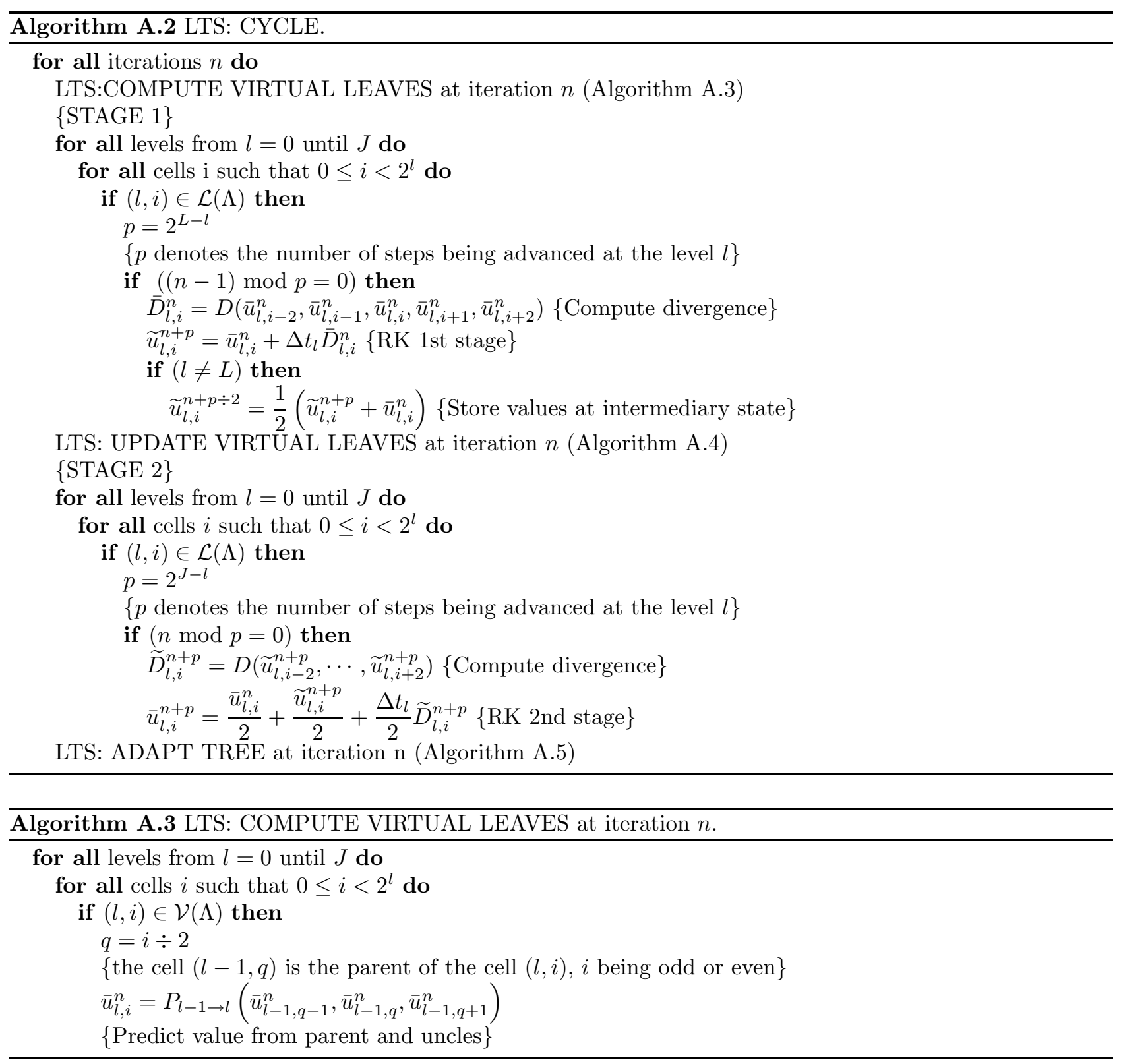

[13] G. Chiavassa and R. Donat. Point value multi-scale algorithms for 2D compressible flow. SIAM J. Sci. Comput., 23(3):805-823, 2001.

[14] A. Cohen. Wavelet methods in numerical analysis. In P. G. Ciarlet and J. L. Lions, editors, Handbook of Numerical Analysis, volume VII. Elsevier, Amsterdam, 2000.

[15] A. Cohen, I. Daubechies, and J. C. Feauveau. Biorthogonal bases of compactly supported wavelets. Comm. Pure Appl. Math., 45:485-560, 1992.

[16] A. Cohen, N. Dyn, S. M. Kaber, and M.Postel. Multiresolution finite volume schemes on triangles. J. Comput. Phys., 161:264286,2000 .

[17] A. Cohen, S. M. Kaber, S. Müller, and M. Postel. Fully adaptive multirresolution finite volume schemes for conservation laws. Math. Comp., 72:183-225, 2003.

[18] A. Cohen, S. M. Kaber, and M. Postel. Adaptive multirresolution for finite volume solutions of gas dynamics. Computer and Fluids, 32:32-38, 2003. 

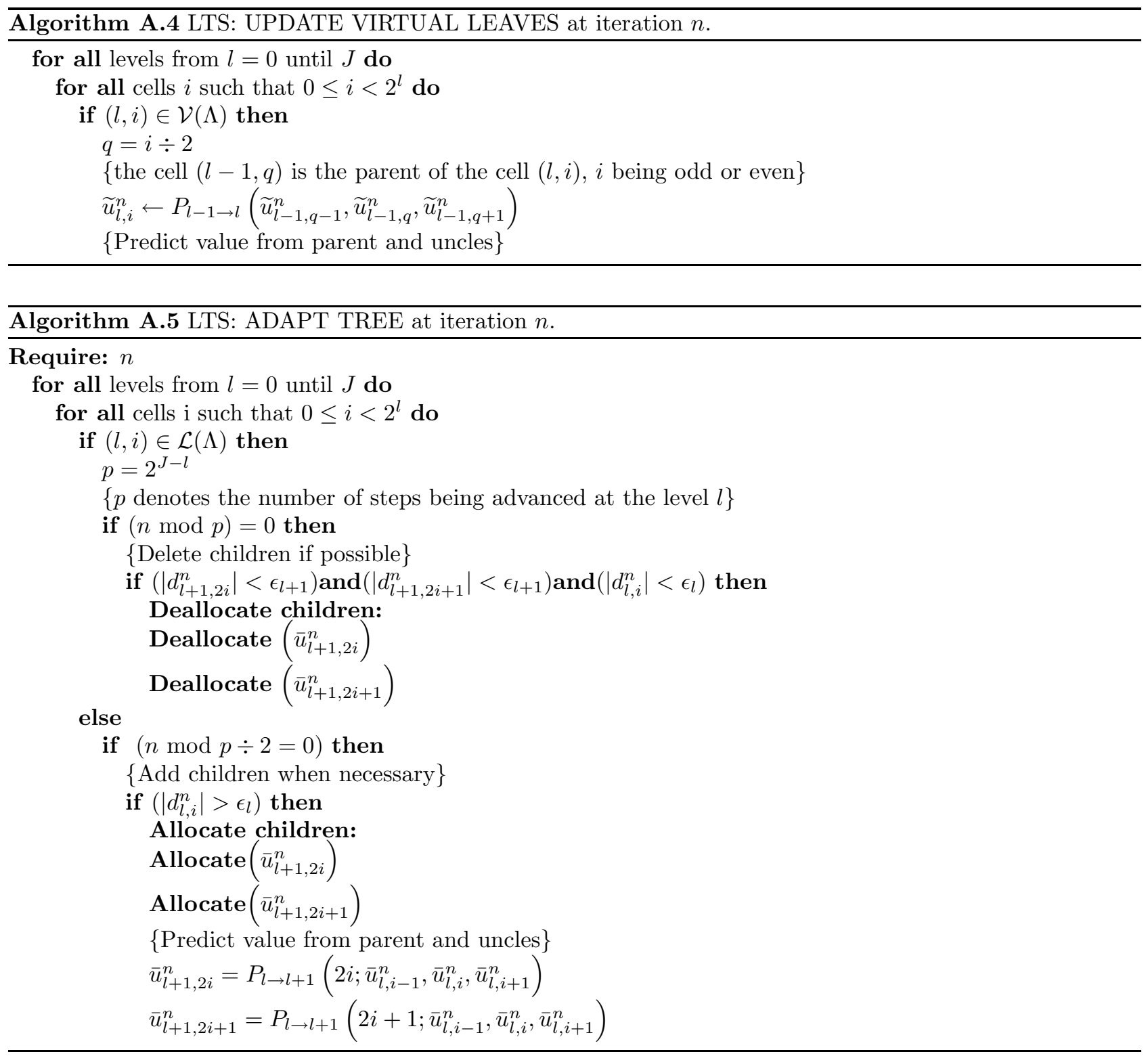

[19] F. Collino, T. Fouquet, and P. Joly. A conservative space-time mesh refinement method for the 1-D wave equation. I. Construction. Numer. Math., 95(2):197-221, 2003.

[20] F. Collino, T. Fouquet, and P. Joly. A conservative space-time mesh refinement method for the 1-d wave equation. II. Analysis. Numer. Math., 95(2):223-251, 2003.

[21] I. Daubechies and W. Sweldens. Factoring wavelet transforms into lifting steps. J. Fourier Anal. Appl., 4(3):247-269, 1998.

[22] C. Dawson and R. Kirby. High resolution schemes for conservation laws with locally varying time steps. SIAM J. Sci. Comput., 22(6):2256-2281, 2001

[23] R. Deiterding. Adaptive mesh refinement methods for conservation laws: theory, implementarion and application. ESAIM Proc., Current volume.

[24] R. Deiterding. Amroc - blockstructured adaptive mesh refinement in object-oriented c++.

[25] R. Deiterding. Construction and application of an amr algorithm for distributed memory computers. In Adaptive Mesh Refinement - Theory and Applications, volume 41 of Lecture Notes in Coputational Science and Engineering, pages 361-372 year $=$. Springer. 
[26] R. Deiterding. Parallel adaptive simulation of multi-dimensional detonation structures. PhD thesis, Brandenburgishe Technishe Universitat Cottbus, Sep 2003.

[27] R. Deiterding, M. O. Domingues, S. M. Gomes, O. Roussel, and K. Schneider. Adaptive multiresolution or adaptive mesh refinement? a case study for 2d Euler equations. ESAIM Proc., 29:28-42, 2009.

[28] G. Deslauriers and S. Dubuc. Symmetric iterative interpolation processes. Constructive Approximation, 5(1):49-68, 1989.

[29] R. DeVore. Nonlinear approximation. Acta Numer., 7:51-150, 1998.

[30] M. O Domingues, P. J. Ferreira, S. M. Gomes, A. Gomide, J. R. Pereira, and P. Pinho. Grid structure impact in sparse point representation of derivatives. J Comput. Appl. Math., 234:237-2389, 2010.

[31] M. O. Domingues, S. M. Gomes, and L. M. A Diaz. Adaptive wavelet representation and differenciation on block-structured grids. Appl. Numer. Math., 47:421-437, 2003.

[32] M. O. Domingues, S. M. Gomes, O. Roussel, and K. Schneider. An adaptive multiresolution scheme with local time stepping for evolutionary PDEs. J. Comput. Phys., 227(8):3758-3780, Apr. 2008.

[33] M. O. Domingues, S. M. Gomes, O. Roussel, and K. Schneider. Space-time adaptive multiresolution methods for hyperbolic conservation laws: Applications to compressible Euler equations. Appl. Numer. Math., 2009.

[34] M. O. Domingues, O. Roussel, and K. Schneider. An adaptive multiresolution method for parabolic PDEs with time-step control. Int. J. Numer. Meth. Eng., 78:652-670, 2009.

[35] D. L. Donoho. Interpolating wavelet transforms. Technical report, Stanford University, 1992.

[36] D. L. Donoho. Smooth wavelet decomposition with blocky coefficient kernels. In Recent Advances in Wavelet Analysis. L. L. Shumaker and G. Webb, 1993.

[37] M. Dumbser, M. Käser, and E. Toro. An arbitrary high order discontinuous Galerkin method for elastic waves on unstructured meshes V: Local time stepping and p-adaptivity. Geophys. J. Int., 171(2):695-717(23), 2006.

[38] M. Farge and K. Schneider. Coherent vortex simulation (CVS), a semi-deterministic turbulence model using wavelets. Flow Turbul. Combust., 66(4):393-426, 2001.

[39] M. Farge, K. Schneider, and N. Kevlahan. Non-gaussianity and coherent vortex simulation for two-dimensional turbulence using an adaptive orthonormal wavelet basis. Phys. Fluids, 11(8):1-15, 1999.

[40] A. Favre. Equations des gaz turbulents compressibles. J. Mécanique, 4:361-421, 1965.

[41] L. Ferm and P. Löstedt. Space-time adaptive solutions of first order PDEs. J. Sci. Comput., 26(1):83-110, 2006.

[42] J. Fröhlich and K. Schneider. An adaptive wavelet-vaguelette algorithm for the solution of PDEs. J. Comput. Phys., 130:174190, 1997.

[43] D. Goldstein and O. Vasilyev. Stochastic coherent adaptive large eddy simulation method. Phys. Fluids, 16:2497-513, 2004.

[44] S. M. Gomes and B. Gustafsson. Combining wavelets with finite differences: a consistency analysis. Preprint, IT, Upssala University, 2002.

[45] D. Gottlieb and E. Turkel. Dissipative two-four methods for time-dependent problems. J. Comput. Phys, 30:703-723, 1976.

[46] A. Harten. Multiresolution algorithms for the numerical solution of hyperbolic conservation laws. Comm. Pure Appl. Math., 48:1305-1342, 1995

[47] A. Harten. Multiresolution representation of data: a general framework. SIAM J. Numer. Anal., 33(3):385-394, 1996.

[48] C. Hirsch. Numerical computation of internal and external flows, volume 2. John Wiley and Sons, 1990.

[49] M. Holmström. Wavelet Based Methods for Time Dependent PDEs. PhD thesis, Uppsala University, 1997.

[50] M. Holmström. Solving hyperbolic PDEs using interpolating wavelets. SIAM J. Sci. Comput., 21(2):405-420, 1999.

[51] M. Kaibara and S. M. Gomes. A fully adaptive multiresolution scheme for shock computations. In E. F. Toro, editor, Godunov Methods: Theory and Applications, pages 597-503. Klumer Academic/Plenum Publishers, 2001.

[52] P. D. Lax and X-D. Liu. Solution of two-dimensional Riemann problems of gas dynamics by positive schemes. SIAM J. Sci. Comput., 19(2):319-340, 1998.

[53] M. Lesieur, O. Metais, and P. Comte. LES of turbulence. Cambridge University Press, 2005.

[54] R. J. LeVeque. Finite Volume Methods for Hyperbolic Systems. Cambridge University Press, 2002.

[55] M.-S. Liou. A sequel to AUSM: AUSM+. J. Comput. Phys., 129:364-382, 1996.

[56] Q. Liu and O. Vasilyev. Brinkman penalization method for compressible flows in complex geometries. J. Comput. Phys., 227(2):946-966, 2007.

[57] S. Mallat. A wavelet tour of signal processing. Academic Press, 1999.

[58] S. Müller. Adaptive multiscale schemes for conservation laws, volume 27 of Lecture Notes in Computational Science and Engineering. Springer-Verlag, Heidelberg, 2003.

[59] S. Müller and Y. Stiriba. Fully adaptive multiscale schemes for conservation laws employing locally varying time stepping. J. Sci. Comput., 30(3):493-531, 2007.

[60] N. Okamoto, K. Yoshimatsu, K. Schneider, M. Farge, and Y. Kaneda. Coherent vortices in high resolution DNS of homogeneous isotropic turbulence : a wavelet viewpoint. Phys. Fluids, 19(115109), 2007.

[61] S. Osher and R. Sanders. Numerical approximations to nonlinear conservation laws with locally varying time space grid. Math. Comp., 43:321-336, 1983

[62] N. Peters and J. Warnatz, editors. Numerical methods in laminar flame propagation, volume 6 of Notes on numerical fluid mechanics. Vieweg, 1982. 
[63] P. Pinho, M. O Domingues, P. J. Ferreira, S. M. Gomes, A. Gomide, and J. R. Pereira. Interpolating wavelets and adaptive finite difference schemes solving Maxwell's equations: gridding effect. IEEE Transactions in Magnetics, 43:1013-1022, 2007.

[64] P. L. Roe. Approximate Riemann solvers, parameter vectors, and difference schemes. J. Comput. Phys., 43:357-372, 1981.

[65] O. Roussel and K. Schneider. A fully adaptive multiresolution scheme for 3D reaction-diffusion equations. In R. Herbin and D. Kröner, editors, Finite Volumes for Complex Applications, volume 3, pages 833-840. Hermes Penton Science, 2002.

[66] O. Roussel and K. Schneider. An adaptive multiresolution method for combustion problems: application to flame ball - vortex interaction. Comp. Fluids, 34(7):817-831, 2005.

[67] O. Roussel and K. Schneider. Coherent vortex simulation of weakly compressible turbulent mixing layers using adaptive multiresolution methods. J. Comput. Phys., 229(6):2267-2286, 2010.

[68] O. Roussel, K. Schneider, and M.Farge. Coherent vortex extraction in 3D homogeneous turbulence: comparison between orthogonal and biorthogonal wavelet decompositions. J. Turbul., 6(11):1-15, 2005.

[69] O. Roussel, K. Schneider, A. Tsigulin, and H. Bockhorn. A conservative fully adaptive multiresolution algorithm for parabolic pdes. J. Comput. Phys., 188:493-523, 2003.

[70] P. Sagaut. Multiscale and Multiresolution Approaches in Turbulence. Imperial College Press, 2006.

[71] K. Schneider and M. Farge. Adaptive wavelet simulation of a flow around an impulsively started cylinder using penalisation. Appl. Comput. Harm. Anal., 12:374-380, 2002.

[72] K. Schneider, M. Farge, G. Pellegrino, and M. Rogers. Coherent vortex simulation of 3D turbulent mixing layers using orthogonal wavelets. J. Fluid Mech., 534:39-66, 2005.

[73] K. Schneider and O. V. Vasilyev. Wavelet methods in computational fluid dynamics. Annu.Rev. Fluid. Mech., 42(42:473503):473-503, 2010.

[74] P. Schroeder and W. Sweldens. Spherical wavelets: efficiently representing functions on the sphere. In Computer Graphics Proceedings (SIGGRAPH 95), pages 161-172, 1995.

[75] C. W. Schulz-Rinne, J. P. Collis, and H. M. Glaz Numerical solution of the Riemann problem for two-dimensional gas dynamics. SIAM J. Sci. Comput., 14:1394-1414, 1993.

[76] E. Süli and D. F. Mayers. An Introduction to Numerical Analysis. Cambridge University Press, 2003.

[77] W. Sweldens. The lifting scheme: a construction of second generation wavelets. SIAM J. Math. Anal., 29:511-543., 1996.

[78] H. Z. Tang and G. Warnecke. A class of high resolution schemes for hyperbolic conservation laws and convection-diffusion equations with varying time and space grids. SIAM J. Sci. Comput., 26(4):1415-1431, 2005.

[79] O. V. Vasilyev and C. Browman. Second generation wavelet collocation method for the solution of partial differential equations. J. Comput. Phys., 165:660-693, 2000.

[80] B. Vreman. Direct and Large-Eddy Simulation of the compressible turbulent mixing layer. PhD thesis, University of Twente, Netherlands, 1995.

[81] B. Vreman, H. Kuerten, and B. Geurts. Large-eddy simulation of the turbulent mixing layer. J. Fluid Mech., 339:357-390, 1997.

[82] J. Walden. A general adaptive solver for hyperbolic PDEs based on filter bank subdivisions. Appl. Numer. Math., 33:317-325, 2000.

[83] P. Wesseling. Principles of Computational Fluid Dynamics. Springer, 2001.

[84] T. Zhang and Y. Zheng. Conjecture on the structure of solutions the Riemann problem for two-dimensional gas dynamics systems. SIAM J. Math. Anal., 21:593-630, 1990.

\section{ACKNOWLEDGMENTS}

M.O. Domingues thankfully acknowledges financial support from the ANR project "M2TFP", Ecole Centrale de Marseille (ECM), Fundação de Amparo a Pesquisa do Estado de São Paulo (FAPESP), and CNPq - the Brazilian Research Council, Brazil. S. Gomes thankfully acknowledges financial support from ECM, CNPq and FAPESP. O. Roussel and K. Schneider acknowledge financial support from the French-German DFG-CNRS Research Program "LES and CVS of Complex Flows". K. Schneider thanks the ANR project "M2TFP" for financial support. We are grateful to Dominique Fougere and Varlei E. Menconni for their helpful computational assistance. 